\title{
Synthesis of Tailored Segmented Polyurethanes Utilizing Continuous Flow Reactors and Real-Time Process Monitoring
}

Xabier Lopez de Pariza, Tim Erdmann*, Pedro L. Arrechea, Leron Perez, Charles Dausse, Nathaniel H. Park, James L. Hedrick, Haritz Sardon*

\section{Content}

1. Materials 2

2. Instrumentation 3

3. Continuous flow reactors 4

3.1. General considerations to step-growth polymerizations in flow reactors 4

$\begin{array}{lll}3.2 . & \text { Flow reactor materials } & 7\end{array}$

3.3. General flow reactor setup 8

4. Calculations 9

4.1. Molecular weight of PEG and PCL-based macrodiols 9

4.2. Monomer conversion by ${ }^{1} \mathrm{H}$ NMR 11

4.3. Fraction of HDO in the polymer 12

$\begin{array}{lll}\text { 4.4. } & \text { Fraction of HEMA in the polymer } & 13\end{array}$

4.5. Percentage of hard segment in the polymer 14

4.6. Molecular weight of HEMA-endcapped PUs 15

$\begin{array}{lll}\text { 5. Characterization details } & 17\end{array}$

$\begin{array}{lll}\text { 5.1. } & 17 \\ 5.2 . & 17 \text { NMR spectra }\end{array}$

$\begin{array}{lll}\text { 5.2. } & \text { FTIR spectra } & 19\end{array}$

6. Catalyst synthesis 20

$\begin{array}{ll}\text { 7. Polymerizations } & 21\end{array}$

7.1. Synthesis of PUs in batch 21

$\begin{array}{ll}\text { 7.1.1. } & \text { Effect of the catalyst on reaction kinetics }\end{array}$

7.1.2. Effect of varying the ratio of the diol monomers on reaction kinetics 23

7.1.3. Control of molecular weight by end-capping with HEMA 25

7.2. Syntheses and experiments using continuous flow reactors 26

7.2.1. Macrodiol PCL2800 26

7.2.2. Catalytic performance of DBTDL and DBU 28

$\begin{array}{ll}\text { 7.2.3. } & \text { In-line ATR-FTIR calibration }\end{array}$

7.2.4. Synthesis of PUs with varying amount of HS 32

7.2.5. Small-scale PU synthesis with end-capping by HEMA 35

7.2.6. Large-scale PU synthesis with end-capping by HEMA 37

7.3. Thermal cross-linking of HEMA-endcapped PUs 39

7.4. UV cross-linking of HEMA-endcapped PUs 40 


\section{Materials}

All chemicals were acquired from Sigma-Aldrich and used as received unless otherwise specified. 4,4'-Methylene diphenyl diisocyanate (MDI), was sublimed at $120{ }^{\circ} \mathrm{C}$ under reduced pressure. The obtained white crystalline solid was stored in a nitrogen-filled glovebox. Macrodiols polyethylene glycol 1500 (PEG1500), polyethylene glycol 2000 (PEG2000), and polycaprolactone diol 2000 (PCL2000) were dried by azeotropic vacuum distillation using anhydrous toluene three times and stored in a $\mathrm{N}_{2}$-filled glovebox. 1,6-Hexanediol (HDO) was stored under vacuum at $40{ }^{\circ} \mathrm{C}$ overnight to remove trace water and, then, in a nitrogen-filled glovebox. 1,8 -Octanediol was sublimed under reduced pressure at $65-70{ }^{\circ} \mathrm{C}$. $\varepsilon$-Caprolactone (CL) was distilled under reduced pressure and stored with activated molecular sieves ( $4 \AA)$ in a nitrogen-filled glovebox. 1,8-Diazabicyclo(5.4.0)undec-7-ene (DBU) was distilled under reduced pressure and stored in a $\mathrm{N}_{2}$-filled glovebox. Dibutyltin dilaurate (DBTDL), 1,5,7triazabicyclo[4.4.0]dec-5-ene (TBD), yttrium (III) isopropoxide oxide (YIPO) and azobisisobutyronitrile (AIBN) were used without further purification. Anhydrous tetrahydrofuran (THF, $>99.9 \%$ ) was stored with activated molecular sieves in a nitrogen-filled glovebox and used for all polymerizations.

Further acronyms/abbreviations: 2-Hydroxyethyl methacrylate (HEMA), butyl acrylate (BuAcr), trimethylolpropane triacrylate (TriAcr), 2,2-dimethoxy-2-phenylacetophenone (DMPA). 


\section{Instrumentation}

All ${ }^{1} \mathrm{H}$ NMR data was collected at $400 \mathrm{MHz}$ using a Bruker Avance 400 Spectrometer at $20{ }^{\circ} \mathrm{C}$ with chemicals shifts reported in ppm. The spectra were referenced to the signal of the residual non-deuterated species of the used solvent: chloroform- $d\left(\mathrm{CDCl}_{3}: \delta\left({ }^{1} \mathrm{H}\right)=7.26 \mathrm{ppm}\right)$, dimethylsulfoxide- $d_{6}\left(\left(\mathrm{CD}_{3}\right)_{2} \mathrm{SO}: \delta\left({ }^{1} \mathrm{H}\right)=2.50 \mathrm{ppm}\right)$.

Attenuated total reflection infrared spectrometry (ATR-FTIR) was performed using a Nicolet iS 5 FTIR Spectrometer at a resolution of $2 \mathrm{~cm}^{-1}$ with a total of 12 to 64 scans. In-line FTIR spectra were recorded with the help of a Golden Gate Micro-Flow Cell Anvil (GS10568) accessory from Speacac.

Molecular weights were analyzed by gel permeation chromatography (GPC) on an Agilent apparatus using a refractive index detector. The measurements were conducted using THF as eluent at $25{ }^{\circ} \mathrm{C}$ and at a flow rate of $0.75 \mathrm{~mL} / \mathrm{min}$. The results are reported relative to a calibration by polystyrene (PS) standards. Chromatograms of PU products were only recorded for monomer conversions of $90 \%$ or higher. GPC measurements were also performed using a Waters Advanced Polymer Chromatography (APC) equipped with a Waters 410 differential refractometer. The set of columns consisted of three Waters ACQUITY APCTMAQ (pore sizes:450/200/125, dp:2.5 $\mu \mathrm{m}$ ). THF was used as the eluent at a flow rate of $0.75 \mathrm{ml} / \mathrm{min}$ and at $25{ }^{\circ} \mathrm{C}$. The APC system was calibrated with polystyrene standards and elution time shifts checked by a $13 \mathrm{kDa}$ PS standard injected with each sample set.

Differential scanning calorimetry (DSC) was performed on a DSC Q2000 (TA Instruments, New Castle, DE) or on a DSC8500 (PerkinElmer, Waltham, WA, USA). Samples were enclosed in aluminum pans and measurements carried out under $\mathrm{N}_{2}$ atmosphere using a heating rate of $20^{\circ} \mathrm{C} / \mathrm{min}$ or $5{ }^{\circ} \mathrm{C} / \mathrm{min}$ and a temperature range from $-70{ }^{\circ} \mathrm{C}$ to $150{ }^{\circ} \mathrm{C}$ or $-75{ }^{\circ} \mathrm{C}$ to 110 ${ }^{\circ} \mathrm{C}$, respectively. 
The gel content of a dried cross-linked sample ( $\sim 0.15 \mathrm{~g})$ was determined by performing Soxhlet extraction for $24 \mathrm{~h}$ using THF, drying the insoluble material remaining in the extraction sleeve at $65^{\circ} \mathrm{C}$ for $24 \mathrm{~h}$, and calculating the weight fraction of the insoluble material with respect to the initial weight. For each crosslinked material the gel content was determined as the average from two individual specimen.

Rheological measurements were carried out with an AR-G2 rheometer (TA Instruments). For dynamic frequency sweep experiments the rheometer was equipped with disposable aluminum plates $(12 \mathrm{~mm})$ and the measurements were performed from 0.01 to $100 \mathrm{~Hz}$ at $20{ }^{\circ} \mathrm{C}$ and at a fixed strain of $1 \%$. Dynamic time sweep experiments were performed at room temperature, under a constant frequency of $1 \mathrm{~Hz}$, and using a UV LED centered at $365 \mathrm{~nm}$ and with its intensity calibrated by an external radiometer. $20 \mathrm{~mm}$ acrylic and aluminum plates were used as bottom and top geometries. The UV light with an intensity of $100 \mathrm{~mW} \cdot \mathrm{cm}^{-2}$ was turned on 1 min after the measurement was started by the software and measurements continued until stabilization of the moduli was observed.

\section{Continuous flow reactors}

\subsection{General considerations to step-growth polymerizations in flow reactors}

In continuous-flow reactors the reaction time is expressed as residence time $(\tau)$ which is the time each fraction of the reaction mixture needs to pass through the reactor. ${ }^{1}$ Therefore, the residence time is determined by the dimensions of the flow reactor and the applied flow rate and can be calculated by Equation S1-S3

$$
\tau=\frac{V_{R}}{Q(t)}(S 1) \quad \tau=\frac{\frac{\pi}{4} \cdot d_{R}^{2} \cdot l_{R}}{Q(t)}[\mathrm{min}](S 2) \quad \tau=\frac{15 \pi \cdot d_{R}^{2} \cdot l_{R}}{Q(t)}[s]
$$

where $V_{R}$ is the reactor volume in $\mathrm{cm}^{3}, Q(t)$ refers to the volumetric flow rate in the reactor in 
$\mathrm{cm}^{3} \cdot \mathrm{min}^{-1}, d_{R}$ is the inner diameter of the reactor in $\mathrm{cm}$ and $l_{R}$ refers to the reactor length in $\mathrm{cm}$.

To successfully perform flow-polymerizations, several parameters have to be taken into account, such as reaction kinetics, residence time, reactor dimensions and volume, viscosity of starting solutions and reaction mixture, mixing efficiency, solubility of potential by-products, and potentially changing fluid characteristics with the progressing reaction. ${ }^{2}$ Here, the optimization of some of these parameters is discussed.

Once the reaction kinetics are determined for a specific reaction, flow conditions can be optimized to obtain the desired conversion as follows:

1. If the selected conditions (equivalent to the batch conditions) showed lower reaction conversion than expected, residence time can be extended by reducing the flow rate $(Q(t))$ or increasing the reactor volume. This is accomplished by increasing the reactor length $\left(l_{r}\right)$ or tubing inner diameter $\left(d_{r}\right.$ or ID). In contrast, higher flow rates or smaller reactor volumes, could be used if the conversion is higher than desired. When varying reactor dimensions and flow rates, the mixing efficiency with regard to the residence time has to be considered as well. ${ }^{3}$ The Reynolds number $(\mathrm{Re})$ presents the ratio of inertial forces to viscous forces and helps to estimate flow characteristics categorized by laminar $(\operatorname{Re}<2000)$, transient $(2000<\operatorname{Re}<4000)$, or turbulent $(\operatorname{Re}>4000)$. At low Re values, the flow is in the laminar regime and mixing is slow due to being dominated by perpendicular diffusion. For fast reactions, high Re values are desired to ensure a turbulent flow leading to fast and efficient mixing of the reactants. The Reynolds number values typical for our reactions do not exceed 100 mainly due to very low flow velocity. ${ }^{4-6}$

With respect to the reaction kinetics, the concentration of the monomers could also be altered within their solubility range to adjust the reaction rate. 
2. To increase the amount of material produced in one experiment, one option is to run the reaction for a longer time. Another option is to increase the reactor volume and

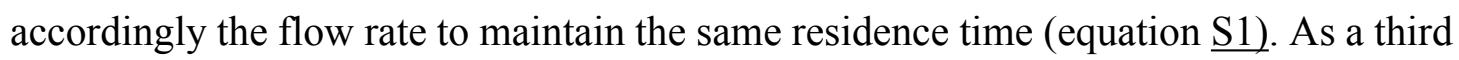
alternative, multiple reactors could also be set up to run in parallel.

Therefore, a good polymerization protocol must consider all the above mentioned.

An important factor to bear in mind when performing polymerization in flow, is the viscosity of the reaction mixture during the polymerization process as it could lead to high pressure changes along the reactor length, precipitation of the product and/or reaction intermediates leading to unstable residence times or even clogging in the system.

For step-growth polymerizations, high conversion is required to obtain high molecular weight polymers according to the Carothers equation. ${ }^{7}$ However, if the reaction in continuous flow is performed at higher concentrations and carried to high conversion, clogging or fouling of the reactor is highly probable. Therefore, the effect of viscosity can be diminished by dilution the feed solutions which, consequently, however will result in longer reaction times based on the rate law.

The initial goal of our work was to prepare segmented PUs in continuous flow at room temperature and within residence times of only a few minutes. In context with the discussion above, in our experiment we used the solubility of the polyols and MDI as guidance to balance monomer concentrations which would allow for a sufficient reaction rate but would not cause clogging of the reactor. We found that a concentration of about $0.48 \mathrm{M}$ for the HDO/polyol solution could be considered high but also afforded stabile HDO/polyol solutions without precipitation. In case of MDI a concentration of $1.92 \mathrm{M}$ was identified as suitable, and the concentration of the catalyst solution was then selected to afford a flow rate in approximately the same order of magnitude. Following this approach and performing catalyst screenings, resulted in reaction conditions fulfilling our initial goals. Viscosity measurements of monomer 
solutions or the reaction mixture were not performed but clogging of the reactor was only observed in reactions without stoichiometric imbalances when the monomer conversion was allowed to reach above $99 \%$. Thus, monitoring and controlling the conversion in a narrow and constant range of up to $98-99 \%$ and/or selectively introducing stoichiometric imbalances were successful strategies to avoid reactor clogging, to synthesize PUs with predefined molecular weights and to increase reproducibility.

\subsection{Flow reactor materials}

All flow reactor materials were purchased from IDEX Health \&Science (OD - outer diameter, ID - inner diameter): Perfluoroalkoxy alkane (PFA) tubing (1507L-PFA Tubing Natural 1/16" OD x 0.040" (1.0 mm) ID; 1512L-PFA Tubing Natural 1/16" OD x 0.020" (0.5 mm) ID; 1902L-PFA+ Tubing Natural 1/16" OD x 0.010" 0.25 mm ID ), connectors (P-249 -Super Flangeless $^{\mathrm{TM}}$ One-Piece Fitting, 1/4-28 Flat-Bottom, for 1/16" OD; or P-235X -Flangeless Nut PEEK, Short, 1/4-28 Flat-Bottom, for 1/16" OD coupled to P-200X -Flangeless Ferrule Tefzel $^{\mathrm{TM}}$ (ETFE), 1/4-28 Flat-Bottom, for 1/16" OD), adapters (P658-Luer Adapter 1/4-28 Female to Female Luer, PEEK), and Tee-mixers (P-714-PEEK Low Pressure Tee Assembly 1/16" PEEK 0.040" thru hole). Luer Lock ${ }^{\mathrm{TM}}$ syringes were purchased from NORM-JECT. The continuous-flow polymerizations were carried out in PhD UltraTM syringe pumps (70-3007) and Pump 33 DDS (Dual Drive System) Syringe Pump (70-3333) from Harvard Apparatus.

\subsection{General flow reactor setup}

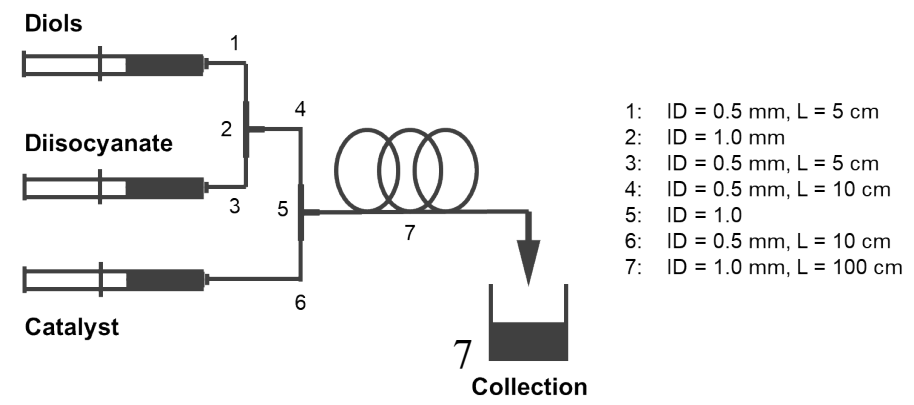


Figure S1: Scheme of a general flow reactor setup used in this work for the preparation of polyurethanes.

From the mentioned commercial source, PFA tubing with an OD of 1/16" is available with IDs of up to $1.0 \mathrm{~mm}$. To help minimize back pressures or clogging in the system, tubing with an ID of $1.0 \mathrm{~mm}$ was used in the flow reactor (Figure S1, 7). To enhance mixing, tubing with an ID of $0.5 \mathrm{~mm}$ (Figure S1, 1, 3, 4, 6) and Tee-mixers with an ID of $1.0 \mathrm{~mm}$ (Figure S1, 2, 5) were chosen to connect the syringes with the reactor tubing. Note, it was experimentally found that Tee-mixers lead to better mixing than cross-mixers and that they reduce the probability of clogging. The diisocyanate, diol and catalyst solutions were prepared in a $\mathrm{N}_{2}$-filled glovebox and loaded into syringes with Luer Lock ${ }^{\mathrm{TM}}$ tip. Additionally, syringes were charged with anhydrous THF to purge the flow reactor setup prior to connecting syringes holding starting materials and catalyst.

The reactor output was directly quenched by precipitation into a vessel containing petroleum ether. Samples were taken for ${ }^{1} \mathrm{H}$ NMR and GPC analysis (only when conversion was above $90 \%$ ). The precipitated crude product was re-dissolved in THF, precipitated into methanol (if not stated otherwise) and isolated by vacuum filtration. Two additional precipitation cycles were performed. The purified products were dried under reduced pressure and characterized by ${ }^{1} \mathrm{H}$ NMR, FTIR and GPC.

\section{Calculations}

\subsection{Molecular weight of PEG and PCL-based macrodiols}

The number-average molecular weights $\left(\mathrm{M}_{\mathrm{n}}\right)$ of the macrodiols (PEG1500, PEG2000, PCL2000 and PCL2800) were calculated from ${ }^{1} \mathrm{H}$ NMR spectra using the end-group integration method. The degree of polymerization (DP) was determined from the ratio between the integral of the terminal hydroxy proton signal (4.58 ppm for PEG or $4.33 \mathrm{ppm}$ for PCL) 
and the methylene proton signal of the repeating unit (3.50 ppm for PEG or $2.27 \mathrm{ppm}$ for PCL) (See Figure S1-S4). The $\mathrm{M}_{\mathrm{n}}$ was calculated by multiplying the DP with the molecular weight of the repeating unit plus the molecular weight of initiator fragment and end-group - water in case of PEG, diethylene glycol in case of PCL2000, and 1,8-octanediol in case of PCL2800.

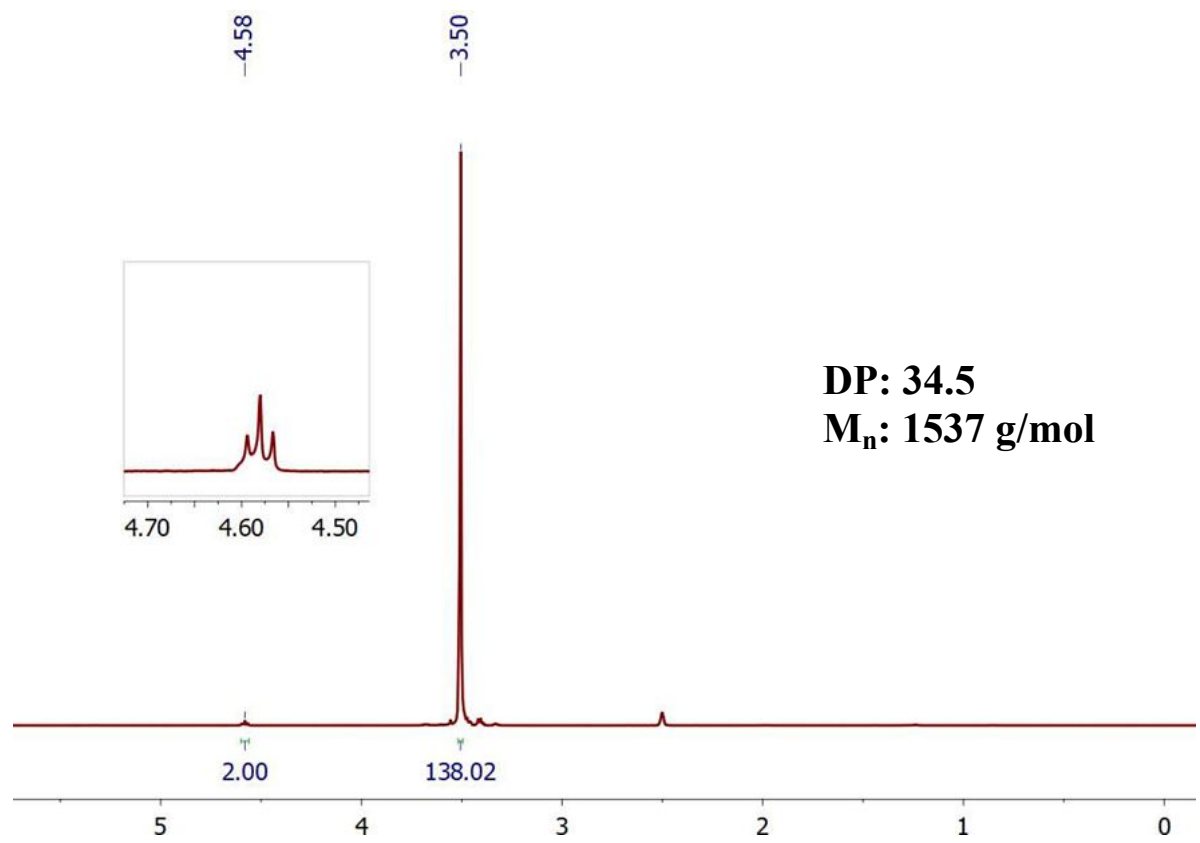

Figure S1: ${ }^{1} \mathrm{H}$ NMR (DMSO- $\left.d_{6}\right)$ of PEG1500.
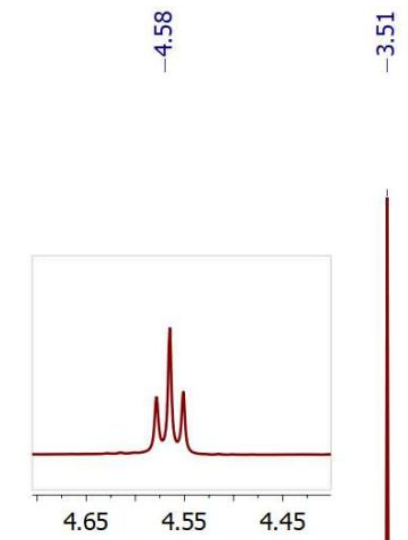

DP: 45

$M_{\mathrm{n}}: 2007 \mathrm{~g} / \mathrm{mol}$

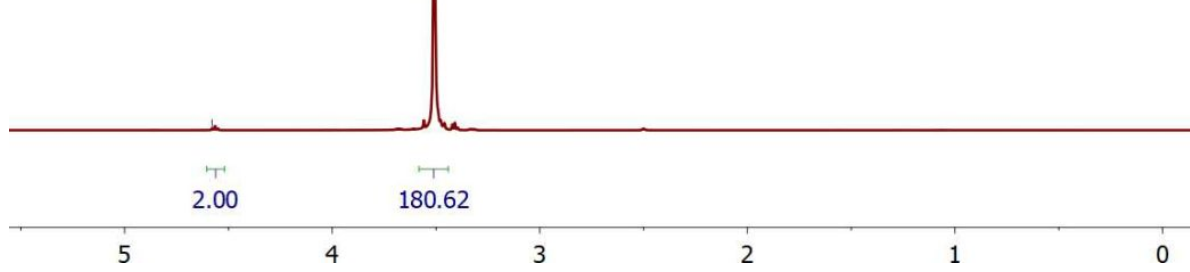

Figure S2: ${ }^{1} \mathrm{H}$ NMR $\left(\mathrm{DMSO}-d_{6}\right)$ of PEG2000. 


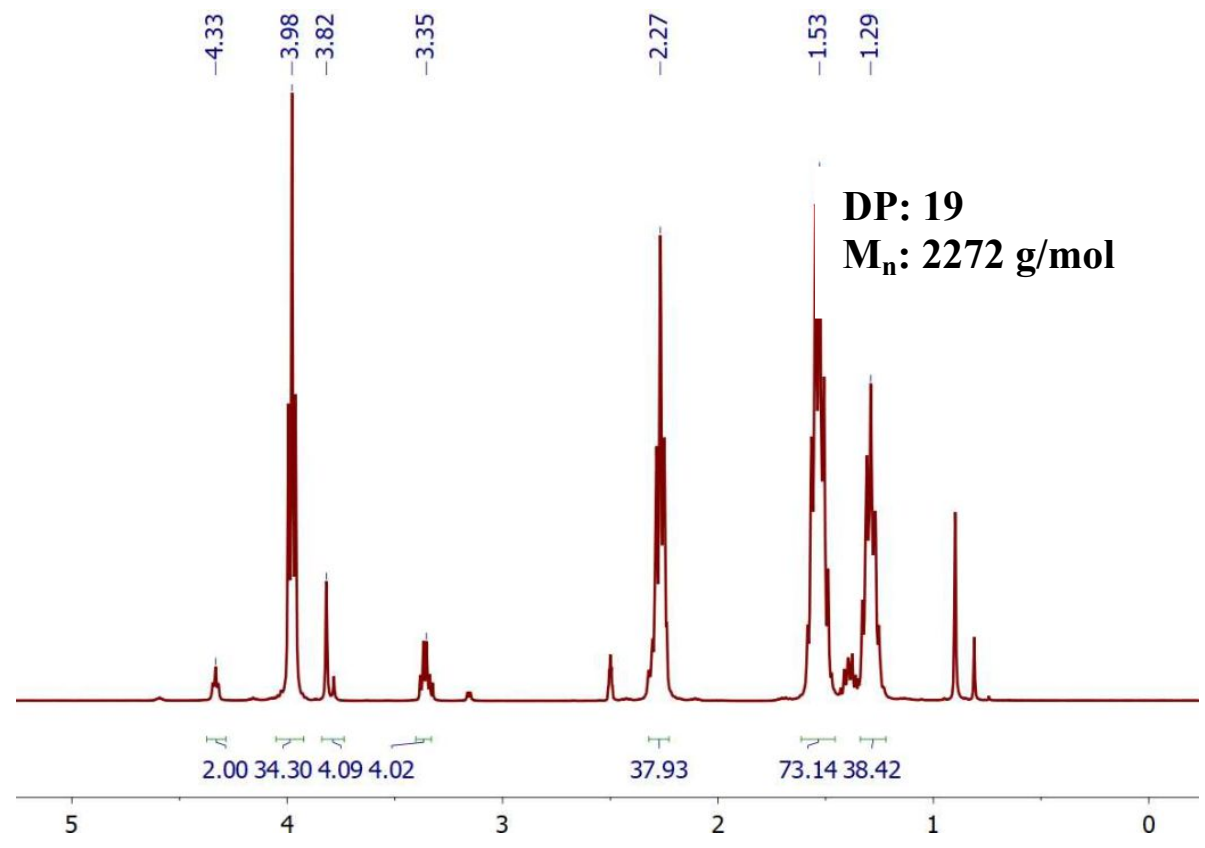

Figure S3: ${ }^{1} \mathrm{H}$ NMR (DMSO- $\left.d_{6}\right)$ of PCL2000.

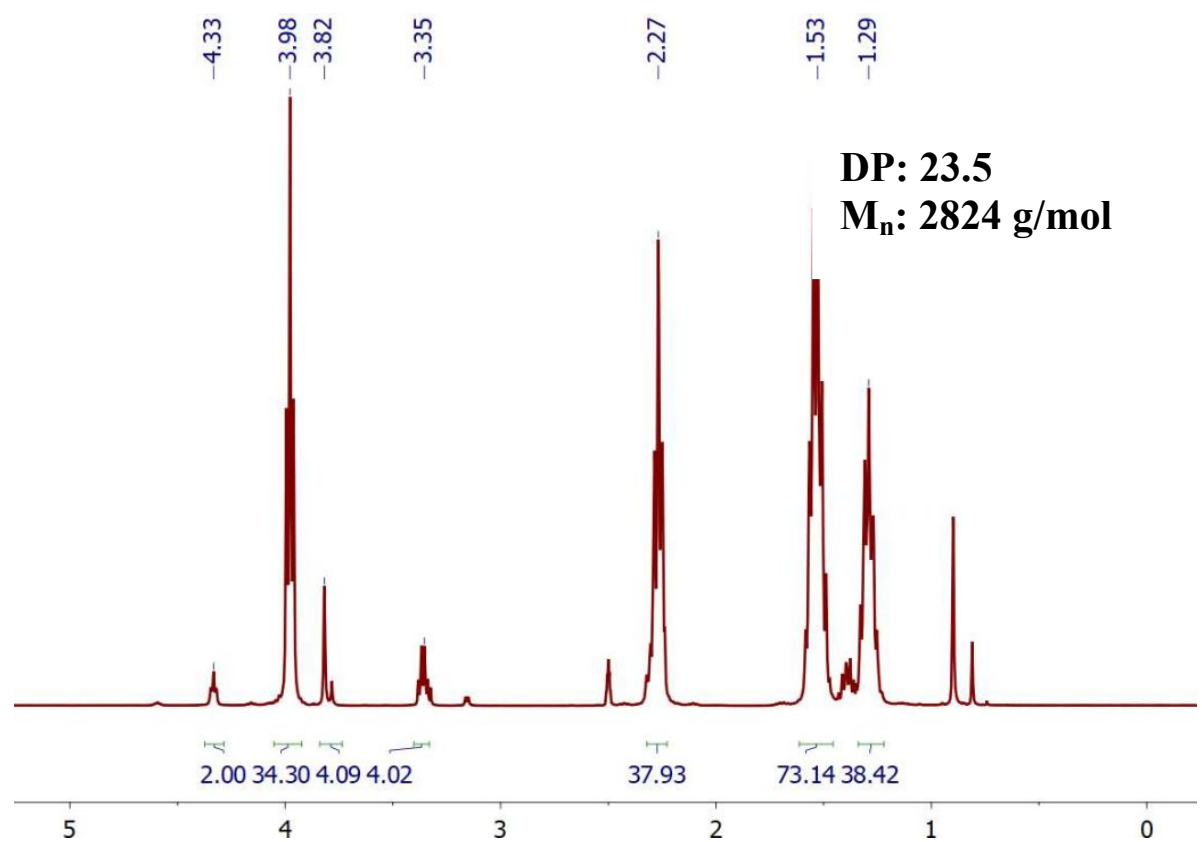

Figure S4: ${ }^{1} \mathrm{H}$ NMR $\left(\mathrm{DMSO}-d_{6}\right)$ of PCL2800.

\subsection{Monomer conversion by ${ }^{1} \mathrm{H}$ NMR}

Reaction conversion was followed by tracking the disappearance of hydroxy protons of the diols and the subsequent appearance of the methylene groups adjacent to the urethane linkage. 
The conversion was calculated by Equation S4 where $A_{P o l,-} \mathrm{CH}_{2}-\mathrm{OCONH}-$ is the area of the polymeric methylene group adjacent to the urethane moiety (4.16 ppm for PEG and $4.03 \mathrm{ppm}$ for PCL and HDO) and $A_{M o n,-O H}$ is the area of the alcohol proton of the diols (4.59 ppm for PEG, 4.36 ppm for PCL and HDO).

$$
\text { Conversion }=\frac{A_{\mathrm{Pol}_{1}-\mathrm{CH}_{2}-\mathrm{OCONH}-}}{A_{\mathrm{Pol},-\mathrm{CH}_{2}-\mathrm{OCONH}-+2 A_{\mathrm{Mon},-\mathrm{OH}}}}
$$

Conversion was calculated separately for each diol and is shown as an average of both according to the presence of each in the polymer. It is worth to mention that this method relies on the resolution of the end-group signals and, therefore, for high molecular weight polymer samples larger errors are introduced as end-group signal intensity is considerably weaker. ${ }^{8}$

\subsection{Fraction of HDO in the polymer}

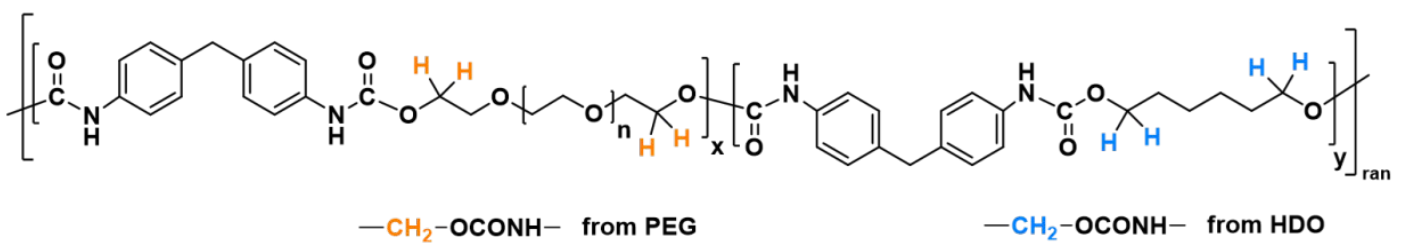

The fraction of HDO in total diols incorporated in the polymer $x_{H D O}$ was determined from the ${ }^{1} \mathrm{H}$ NMR spectrum of the purified product by the integral intensity of signals corresponding to the methylene groups attached to the urethane linkage originating from the different diols. In case of the polymers containing PEG and HDO $x_{H D O}$ can be determined by

$$
x_{H D O}=\frac{A_{H D O,-C_{2}-O C O N H-}}{A_{P E G,-\mathrm{CH}_{2}-O C O N H-}+A_{H D O,-\mathrm{CH}_{2}-O C O N H-}}
$$

where $A_{H D O}-C_{2}-O C O N H$ - is centered at $4.03 \mathrm{ppm}$ and $A_{P E G,-C H_{2}-O C O N H}$ at $4.16 \mathrm{ppm}$. 


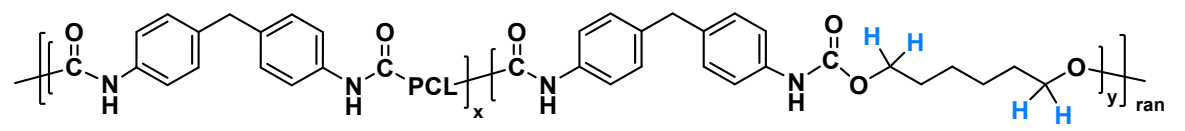

PCL:

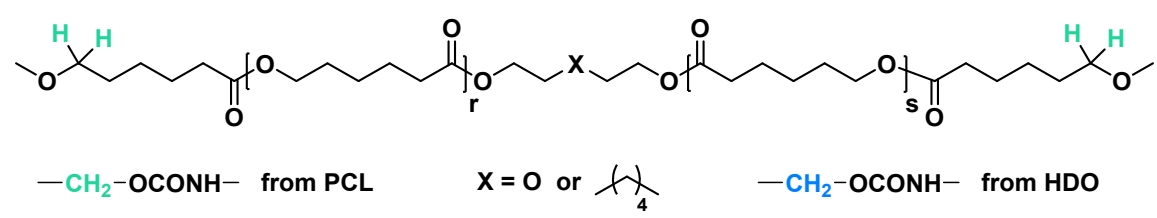

In ${ }^{1} \mathrm{H}$ NMR spectra for PUs containing PCL and HDO the area at 4.10-3.90 ppm $\left(A_{P C L}+H_{D O},-\mathrm{CH}_{2}-\mathrm{OCONH}-\right)$ shows an overlap of the signals corresponding to PCL and HDO methylene protons next to the urethane group, respectively (Figure S7, signal 9 and 12). For this reason, a straightforward calculation of $x_{H D O}$ is not possible but considering the experimentally determined degree of polymerization $D P_{N M R}$ and the area of the signal at 2.27 ppm corresponding to PCL methylene protons next to the PCL carbonyl carbon atom $A_{P C L}, C_{2}-\mathrm{CO}-($ Figure S7, signal 16) Equation S6 was derived.

$$
x_{H D O}=1-\frac{2}{2+D P_{N M R}\left(\frac{A_{P C L}+H D O,-C H_{2}-O C O N H-}{A_{P C L,}-C_{2}-C O-}-1\right)}
$$

The percentage of HDO was further used to determine the percentage of hard segment in the polymers (Equation S9).

\subsection{Fraction of HEMA in the polymer}

Due to the equimolar ratio of functional groups participating in the polyaddition, the theoretical molar fraction of HEMA in total diols $x_{H E M A, t h}$ can be calculated by Equation S7. Its relation to the molar fraction of HEMA hydroxyl groups in total hydroxyl groups $x_{O H, H E M A}$ is expressed in Equation S8. 


$$
\begin{array}{r}
x_{H E M A, t h}=\frac{n_{H E M A}}{n_{M D I}+\frac{n_{H E M A}}{2}} \\
x_{H E M A, t h}=\frac{2 x_{O H, H E M A}}{x_{O H, H E M A}+1}
\end{array}
$$

The molar fraction of HEMA in the polymer $x_{H E M A, \exp }$ was determined from the ${ }^{1} \mathrm{H}$ NMR spectrum of the purified product by integrating the areas corresponding to the peaks of the methylene groups of from the diol moieties and HEMA attached to the urethane linkage.

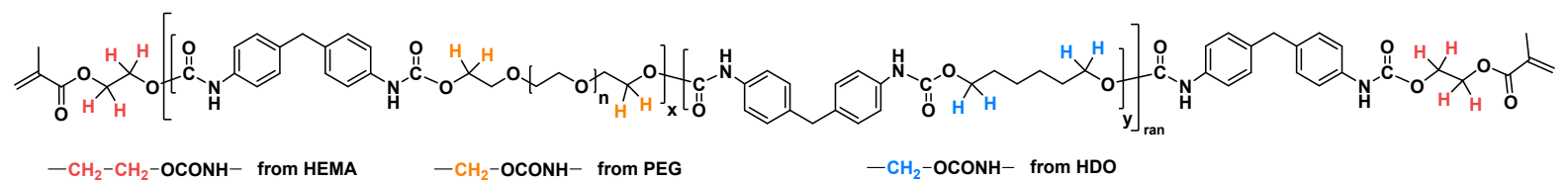

In case of polymers containing PEG, HDO and HEMA $x_{H E M A, \exp }$ was calculated by

$$
\begin{aligned}
& x_{H E M A, \exp } \\
& =\frac{A_{H E M A,-\mathrm{CH}_{2}-\mathrm{CH}_{2}-\mathrm{OCONH}-}}{A_{P E G}-\mathrm{CH}_{2}-\mathrm{OCONH}-+A_{\mathrm{HDO},-\mathrm{CH}_{2}-\mathrm{OCONH}-}+A_{\mathrm{HEMA},-\mathrm{CH}_{2}-\mathrm{CH}_{2}-\mathrm{OCONH}-}}
\end{aligned}
$$
$(S 9)$

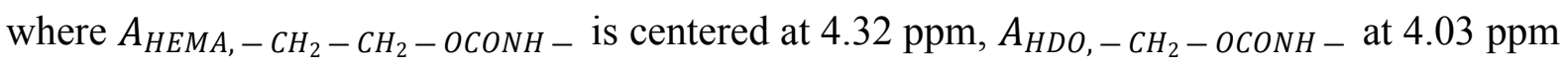
and $A_{P E G,-C H_{2}-O C O N H}$ - at $4.16 \mathrm{ppm}$.

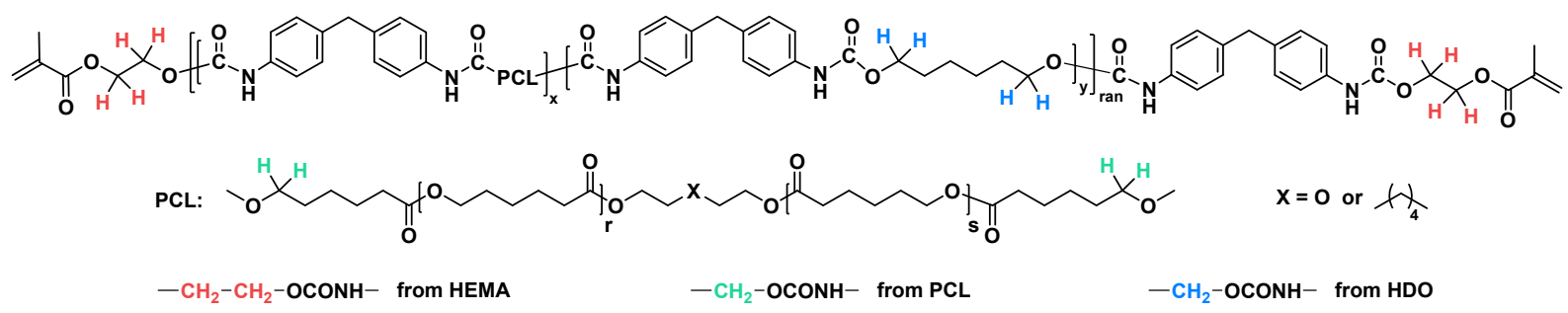

For polymers containing PCL, signals of the polymeric PCL and HDO-based methylene groups next to the urethane unit overlap in the ${ }^{1} \mathrm{H}$ NMR spectrum. Therefore, $x_{H E M A}$ was calculated by 


$$
x_{H E M A, \exp }=\frac{A_{H E M A,}-\mathrm{CH}_{2}-\mathrm{CH}_{2}-\mathrm{OCONH}-}{A_{P C L}+\mathrm{HDO},-\mathrm{CH}_{2}-\mathrm{OCONH}-+A_{\mathrm{HEMA},-\mathrm{CH}_{2}-\mathrm{CH}_{2}-\mathrm{OCONH}-}}
$$

where $A_{P C L}+H D O,-\mathrm{CH}_{2}-\mathrm{OCONH}-$ is located at $4.03 \mathrm{ppm}$ and $\mathrm{A}_{\mathrm{HEMA}}-\mathrm{CH}_{2}-\mathrm{CH}_{2}-\mathrm{OCONH}-$ at $4.32 \mathrm{ppm}$ (Equation S10).

\subsection{Percentage of hard segment in the polymer}

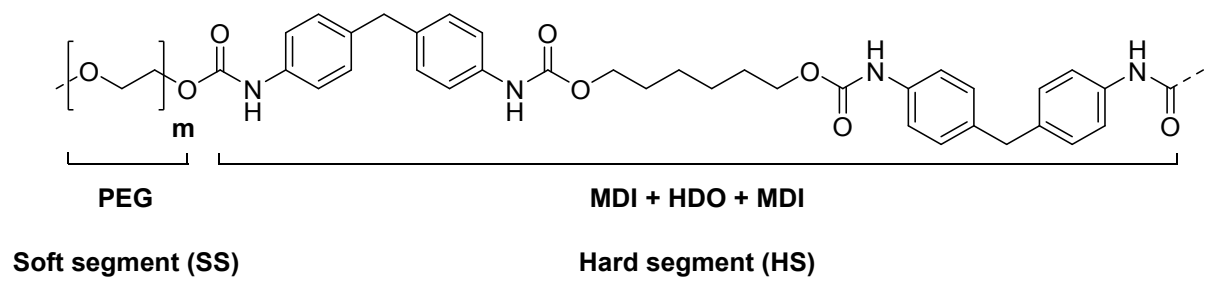

Figure S5: Section of a segmented linear PU backbone showing soft segment, PEG, and hard segment, MDI-HDO-MDI.

Segmented polyurethanes were synthesized from diols with significantly different thermal properties. This leads to the formation of segments along the polymer chain with either glass transition temperatures well below or melting temperatures well above ambient conditions. Accordingly, these segments are named soft segment (SS) and hard segment (HS) (Figure S5). In this work the HS is formed by MDI and HDO, ${ }^{9}$ and the corresponding hard segment weight fraction $w_{H S}$ was calculated by:

$$
w_{H S}=\frac{M_{M D I}+x_{H D O} M_{H D O}}{M_{M D I}+x_{H D O} M_{H D O}+\left(1-x_{H D O}\right) M_{\text {macrodiol }}}
$$

where $M_{M D I}$ is the molecular weight of MDI, $M_{H D O}$ is the molecular weight of HDO, $M_{\text {macrodiol }}$ is the molecular weight of the macrodiol and $x_{H D O}$ is the mol fraction of HDO in the polymer. 


\subsection{Molecular weight of HEMA-endcapped PUs}

According to Carothers equation ${ }^{7}$ the number-average degree of polymerization $\bar{X}_{n}$ for stepgrowth polymerizations of bifunctional monomers A-A and B-B is directly related to the extent of the reaction and the stoichiometry between the functional groups, ${ }^{10}$

$$
\bar{X}_{n}=\frac{1+r}{1+r-2 r p}
$$

where $p$ is the extent of the reaction and $r$ the stoichiometric imbalance calculated by the ratio of total underrepresented functional groups (A) $N_{A}$ to total functional groups present in an excess (B) $N_{B}$.

Incorporating a monofunctional compound B while maintaining a stoichiometric imbalance of 1 (e.g. 1 mol A-A, 0.95 mol B-B, 0.1 mol B) will limit $\bar{X}_{n}$ even though the conversion of the reaction reaches completeness. ${ }^{7}$ In this case the degree of polymerization is additionally depending on average functionality of monomers in the system $\left(f_{\text {avg }}\right)$ defined by

$$
f_{\text {avg }}=\frac{\sum N_{i} f_{i}}{\sum N_{i}}(S 13)
$$

where $N_{i}$ is the amount of monomer $i$ with functionality $f_{i}$. Considering $p, \bar{X}_{n}$ can then be calculated by Equation S14.

$$
\bar{X}_{n}=\frac{2}{2-p f_{a v g}}
$$

With $\bar{X}_{n}$ and the molecular weight of the repeating unit $M_{r u}$, the number-average molecular weight $\bar{M}_{n}$ is accessible by

$$
\bar{M}_{n}=\frac{\bar{X}_{n} \cdot M_{r u}}{2}(S 15)
$$


where $M_{r u}$ was calculated by the feed ratio-adjusted average of the molecular weights of the monomers. Since in our work HEMA was used as monofunctional alcohol to decrease $f_{\text {avg }}$ below 2 and, thus, to limit $\bar{M}_{n}$ Equations S13-S15 can be rewritten as follows.

$$
\begin{gathered}
f_{\text {avg }}\left(x_{H E M A}\right)=\frac{2-x_{H E M A}}{1-\frac{x_{H E M A}}{4}}(S 16) \\
\bar{X}_{n}\left(x_{H E M A}, p\right)=\frac{4-x_{H E M A}}{2 p\left(x_{H E M A}-2\right)-x_{H E M A}+4} \\
\lim _{p \rightarrow 1} \bar{X}_{n}\left(x_{H E M A}, p\right)=\frac{4-x_{H E M A}}{x_{H E M A}} \quad(S 18) \\
\bar{M}_{n}\left(x_{H E M A}, p\right)=\frac{M_{r u}\left(4-x_{H E M A}\right)}{4 p\left(x_{H E M A}-2\right)-2 x_{H E M A}+8} \\
\lim _{p \rightarrow 1} \bar{M}_{n}\left(x_{H E M A}, p\right)=\frac{M_{r u}\left(4-x_{H E M A}\right)}{2 x_{H E M A}} \quad(S 20)
\end{gathered}
$$

Finally, with Equation S8 results the relationship between $\bar{M}_{n}$ and the molar fraction of HEMA hydroxyl groups in total hydroxy groups in the system.

$$
\begin{gathered}
\bar{M}_{n}\left(x_{O H, H E M A}, p\right)=\frac{M_{r u}\left(x_{O H, H E M A}+2\right)}{2 x_{O H, H E M A}-4 p+4} \\
\lim _{p \rightarrow 1} \bar{M}_{n}\left(x_{O H, H E M A}, p\right)=\frac{M_{r u}\left(x_{O H, H E M A}+2\right)}{2 x_{O H, H E M A}}
\end{gathered}
$$

If the polyaddition is executed to quantitative monomer conversion Equation S20 and S22 help to directly estimate the limit of $\bar{M}_{n}$ with $x_{H E M A}$ or $x_{O H, H E M A}$, respectively.

\section{Characterization details}

\section{$5.1 \quad{ }^{1} \mathrm{H}$ NMR spectra}


All PUs containing different PEG:HDO molar ratios showed similar ${ }^{1} \mathrm{H}$ NMR spectra (Figure S6). Urethane-NH protons attached to PEG (5) and HDO (5') were observed at 9.66 ppm and $9.51 \mathrm{ppm}$, respectively. MDI aromatic protons were assigned to signals at $7.33 \mathrm{ppm}$ and $7.09 \mathrm{ppm}(\mathbf{6}, 7)$. PEG alcohol protons OH appeared at $4.59 \mathrm{ppm}$ (1) and HDO alcohol protons $\mathrm{OH}$ at $4.36 \mathrm{ppm}$ (15). $\mathrm{PEG}$ methylene groups $\mathrm{CH}_{2}$ attached to the urethane moiety were observed at $4.16 \mathrm{ppm}$ (4) and $\mathrm{HDO}$ methylene protons $\mathrm{CH}_{2}$ at $4.03 \mathrm{ppm}$ (9). MDI methylene protons Ar- $\mathrm{CH}_{2}$-Ar were noted at $3.77 \mathrm{ppm}(\mathbf{8})$ and methylene protons of PEG $\alpha$ to urethane $\mathrm{CH}_{2}$ at 3.62 ppm (3). PEG methylene protons $\mathrm{CH}_{2}$ of the repeating unit were assigned to $3.50 \mathrm{ppm}\left(\mathbf{2}, \mathbf{2}^{\prime}\right)$. HDO methylene groups $\mathrm{CH}_{2}$ attached to the hydroxyl moiety appeared at $3.40 \mathrm{ppm}(\mathbf{1 4})$ and remaining methylene protons $\mathrm{CH}_{2}$ from HDO between $1.61 \mathrm{ppm}$ and 1.37 ppm (10,11,12,13) (Figure S6).

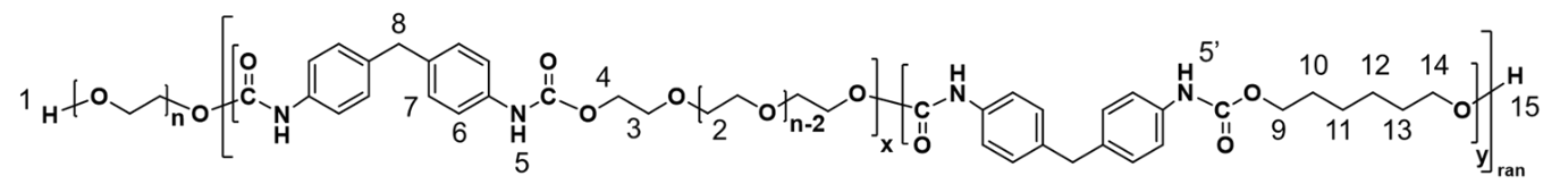

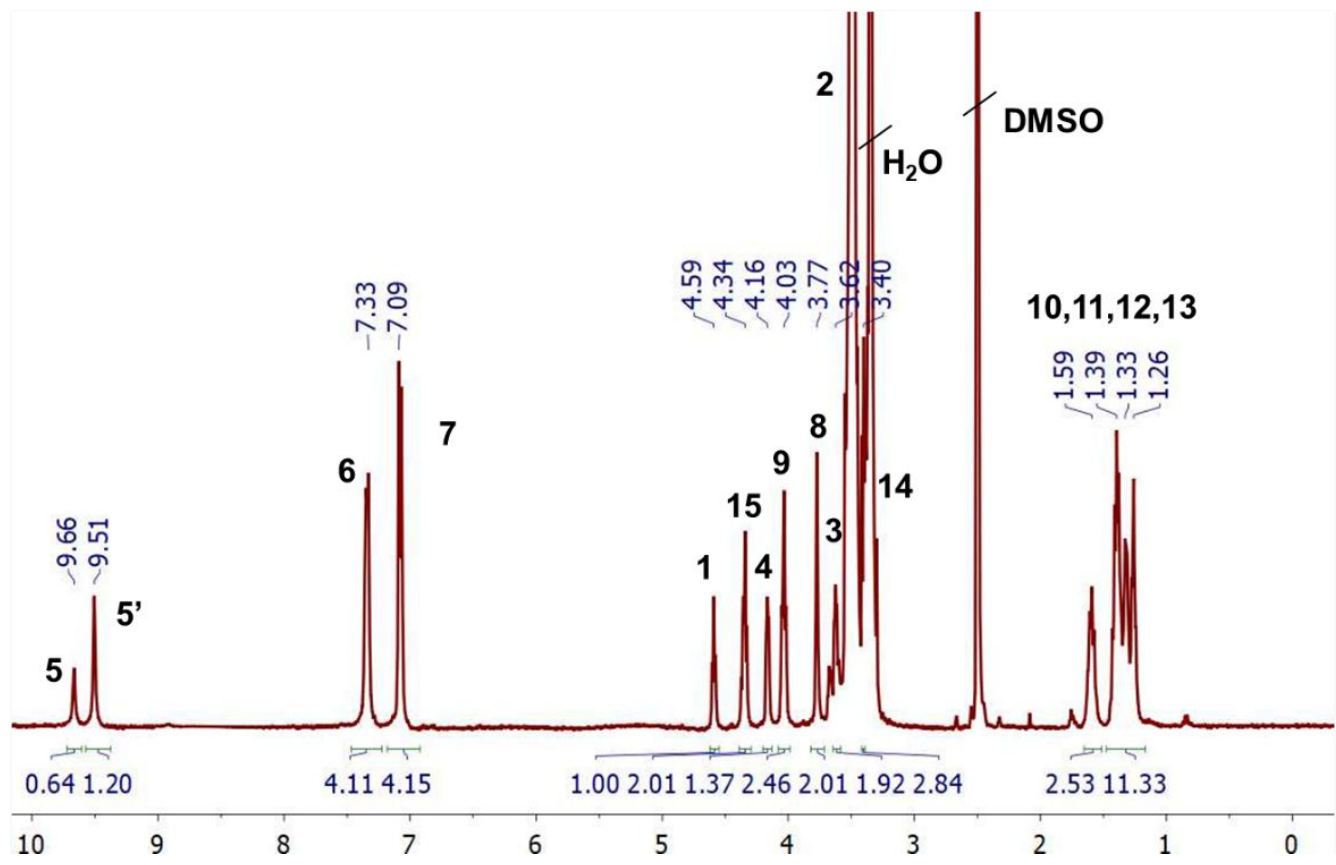

Figure S6: ${ }^{1} \mathrm{H}$ NMR spectra of BatchPU1 after 10 min reaction time. 
${ }^{1} \mathrm{H}$ NMR spectra of PUs containing PCL as macrodiol and HEMA as end groups showed a high similarity to the PEG counterpart discussed above (Figure S7). Urethane-NH protons attached to HEMA and PCL/HDO were observed at $9.66 \mathrm{ppm}$ and $9.50 \mathrm{ppm}$, respectively $(\mathbf{5}, \mathbf{5}, \mathbf{5}$ '). MDI aromatic protons appeared at $7.34 \mathrm{ppm}$ and $7.08 \mathrm{ppm}(\mathbf{6}, \mathbf{7})$. HEMA alkene protons $\mathrm{CH}$ were found at $6.04 \mathrm{ppm}$ and $5.69 \mathrm{ppm}\left(\mathbf{1}, \mathbf{1}^{\prime}\right)$ and HEMA methylene protons at $4.32 \mathrm{ppm}(\mathbf{3}$, 4). PCL and HDO methylene groups $\mathrm{CH}_{2}$ attached to the urethane moiety were assigned to signals at $4.04 \mathrm{ppm}\left(\mathbf{9}, \mathbf{1 2}\right.$ '). PCL methylene protons $\mathrm{CH}_{2}$ of the repeating unit were noted at 3.97 ppm (12). Methylene protons $\mathrm{CH}_{2}$ of the diethylene glycol (used as initiator for the synthesis of PCL) substructure were observed at $3.80 \mathrm{ppm}$ and $3.34 \mathrm{ppm}(\mathbf{1 7}, \mathbf{1 8})$. MDI methylene protons $\mathrm{Ar}-\mathrm{CH}_{2}$-Ar protons were present at $3.77 \mathrm{ppm}(\mathbf{8})$ and methylene protons of PCL $\alpha$ to the ester linkage $\mathrm{CH}_{2}$ at $2.26 \mathrm{ppm}$ (16). HEMA $\mathrm{CH}_{3}$ protons appeared at $1.87 \mathrm{ppm}$ (2) and the remaining methylene protons $\mathrm{CH}_{2}$ of PCL and HDO between $1.61 \mathrm{ppm}$ and 1.28 ppm $(\mathbf{1 0}, \mathbf{1 1}, \mathbf{1 3}, \mathbf{1 4}, \mathbf{1 5})$ (Figure $\mathbf{S 7})$. 

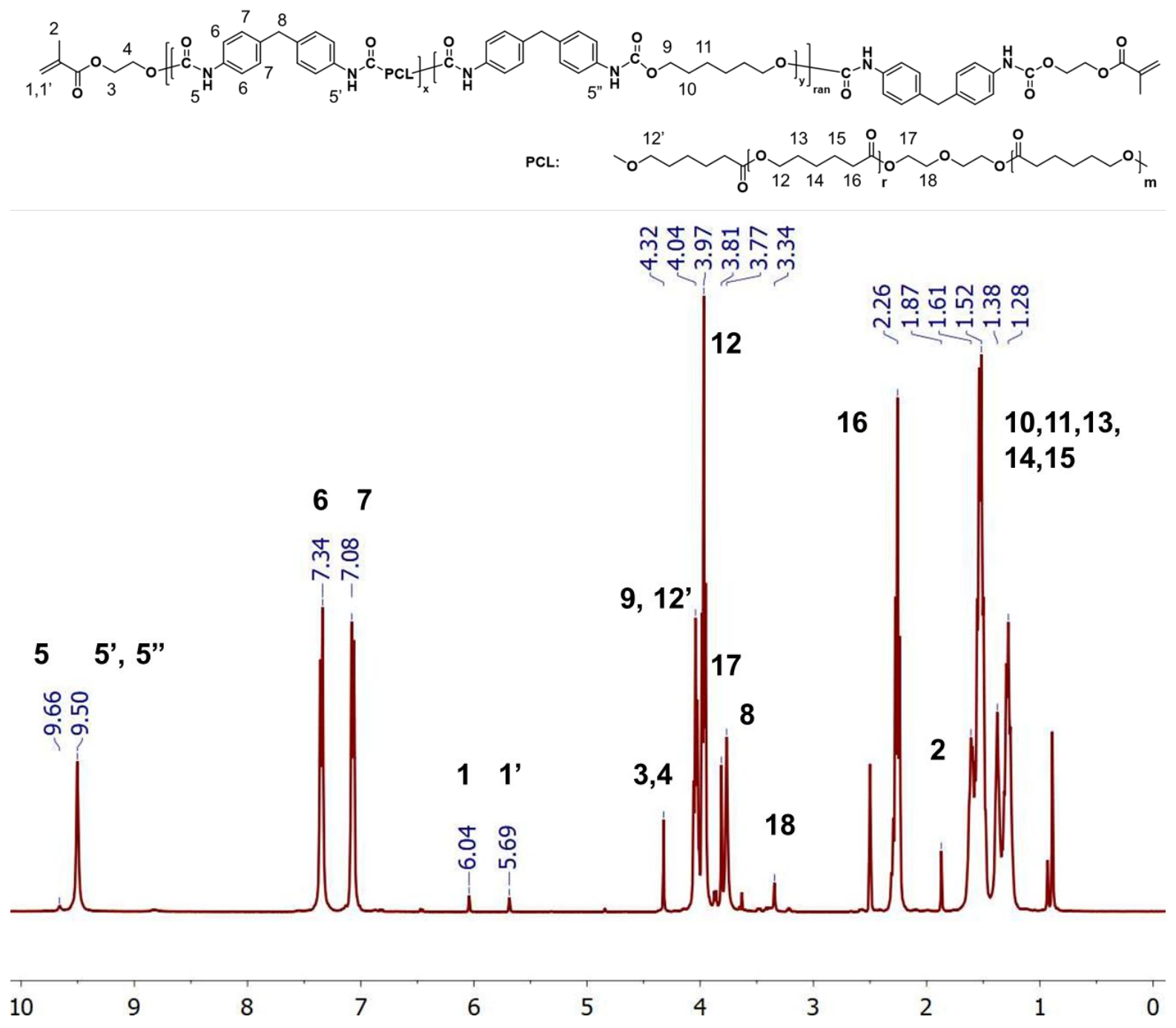

Figure S7: ${ }^{1} \mathrm{H}$ NMR spectra of FlowPU8 after 10 min reaction time.

\subsection{FTIR spectra}

The analysis of the purified PUs by FTIR showed similar spectra in all cases (Figure S8). The characteristic polyurethane band related to the $\mathrm{N}-\mathrm{H}$ stretching under the presence of hydrogen bonding between urethane moieties appeared at $3317 \mathrm{~cm}^{-1}$. Bands indicative for the urethane carbonyl $(\mathrm{C}=\mathrm{O})$ stretching under the presence of hydrogen bonding were observed at $1723 \mathrm{~cm}^{-1}$ and at $1698 \mathrm{~cm}^{-1}$ for amorphous and ordered domains, respectively. Another polyurethanespecific characteristic is the $\mathrm{C}-\mathrm{N}$ deformation band which was noted at $1530 \mathrm{~cm}^{-1}$. Bands from 
the isocyanate stretching vibration usually appear around $2200-2300 \mathrm{~cm}^{-1}$ and were not observed confirming the absence of unreacted isocyanate groups.

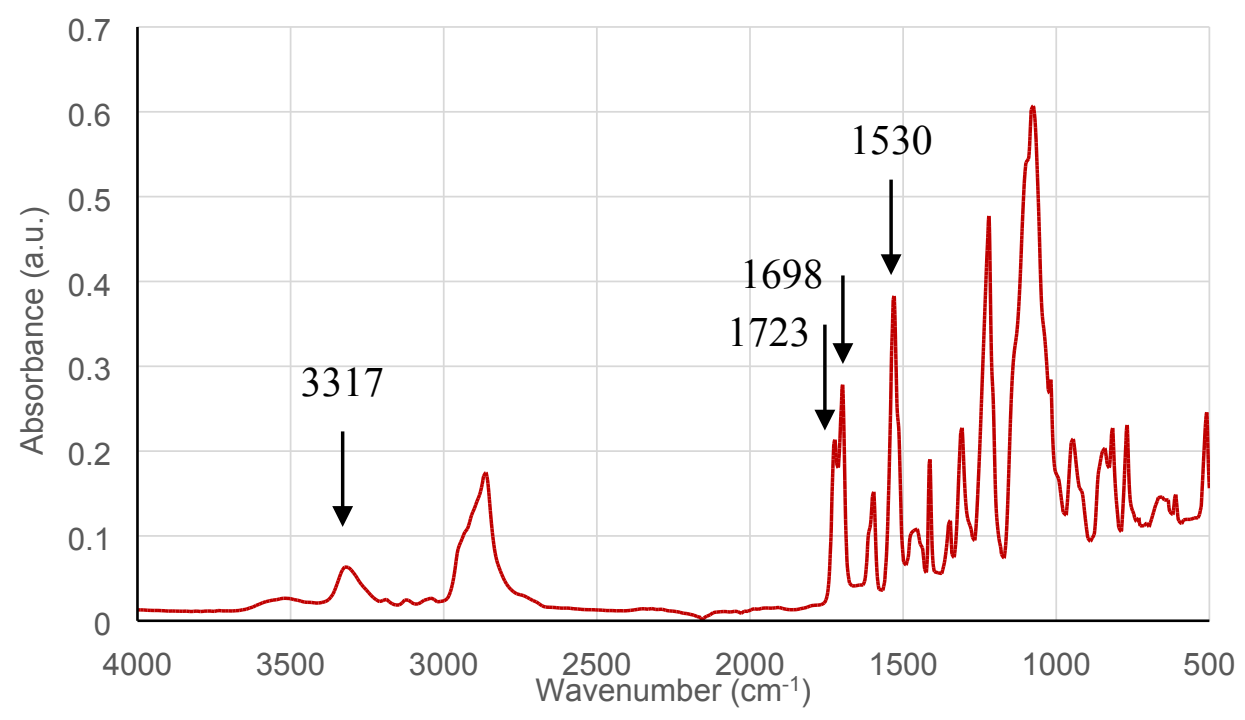

Figure S8: FTIR spectra of BatchPU6.

\section{Catalyst synthesis}

\section{$\mathrm{CF}_{3} \mathrm{PhUPh}$}

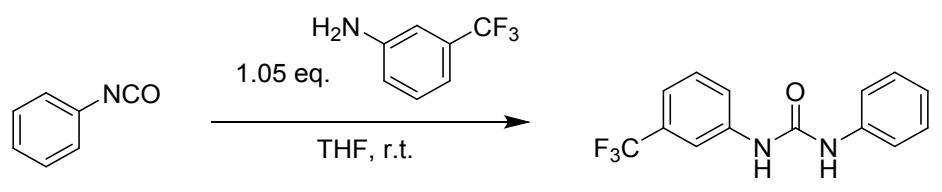

To a solution of $12.08 \mathrm{~g}$ (75.00 mmol, 1.05 eq.) 3-(trifluoromethyl)aniline in $200 \mathrm{~mL}$ THF $8.51 \mathrm{~g}$ (71.40 mmol, 1.00 eq.) phenyl isocyanate were added dropwise over $5 \mathrm{~min}$. After stirring overnight, the mixture was concentrated under a stream of air. Solids were precipitated by adding hexane, separated by vacuum filtration, re-dissolved in THF and precipitated again by adding hexane. This procedure was repeated two additional times before the crude material was decolorized by adding activated carbon to a solution in THF. Afterwards, activated carbon was removed by passing the suspension through a $0.2 \mu \mathrm{m}$ PTFE filter. After evaporating the 
solvents under reduced pressure the solids were recrystallized from IPA, affording the product, 1-phenyl-3-(3-(trifluoromethyl)phenyl)urea, as white powder $(16.24 \mathrm{~g}, 81 \%) .{ }^{1} \mathrm{H}$ NMR $\left(\mathrm{DMSO}-d_{6}\right): \delta[\mathrm{ppm}]=9.05(1 \mathrm{H}, \mathrm{s}), 8.80(1 \mathrm{H}, \mathrm{s}), 8.03(1 \mathrm{H}, \mathrm{s}), 7.56(1 \mathrm{H}, \mathrm{d}), 7.54-7.42(3 \mathrm{H}$, m), $7.29(3 \mathrm{H}, \mathrm{m}), 6.99(1 \mathrm{H}, \mathrm{t}) .^{11}$

\section{Polymerizations}

\subsection{Synthesis of PUs in batch}

\subsubsection{Effect of the catalyst on reaction kinetics}

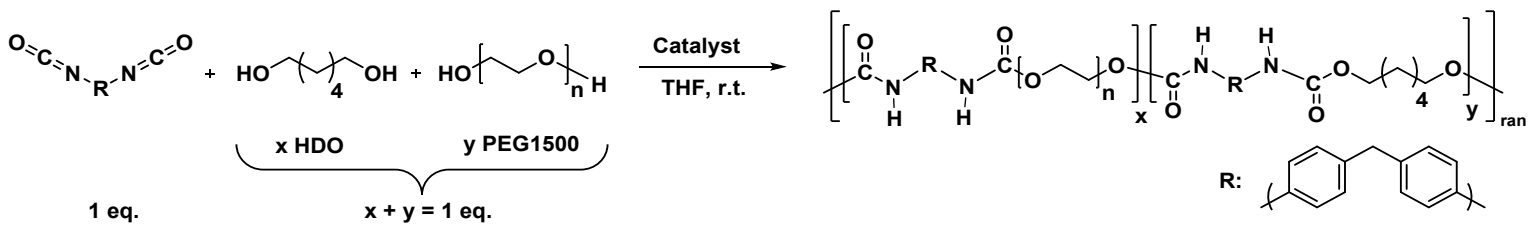

In a $\mathrm{N}_{2}$-filled glovebox a $0.48 \mathrm{M}$ solution of PEG1500:HDO (40:60) was prepared by dissolving $2.70 \mathrm{~g}$ (1.76 mmol, 0.40 eq.) PEG1500 and $312 \mathrm{mg}$ (2.64 mmol, 0.60 eq.) HDO in anhydrous THF to a total volume of $9.2 \mathrm{~mL}$. A $1.92 \mathrm{M}$ MDI solution was prepared by dissolving $1100 \mathrm{mg}$ (4.40 mmol, 1.00 eq.) MDI in anhydrous THF to a total volume of $2.3 \mathrm{~mL}$ and a solution of $0.01 \mathrm{~mol}-\%$ DBTDL (with regard to MDI) by dissolving $0.03 \mathrm{mg}$ $\left(4.8 \cdot 10^{-5} \mathrm{mmol}, 1 \cdot 10^{-4}\right.$ eq. $)$ in $0.25 \mathrm{~mL}$ anhydrous THF. All catalyst solutions used in the screening experiment are listed in Table $\mathbf{S 1}$.

Table S1: Catalyst solutions for catalyst screening experiments.

\begin{tabular}{lcccccc}
\hline Entry & Catalyst & $\mathbf{m o l - \%}$ to MDI & eq. to MDI & $\mathbf{n}[\mathbf{m m o l}]$ & $\mathbf{m}[\mathbf{m g}]$ & $\mathbf{V}_{\text {THF }}(\mathbf{m L})$ \\
\hline BatchPU1 & - & - & - & - & - & 0.25 \\
BatchPU2 & DBTDL & 0.01 & $1 \cdot 10^{-4}$ & $4.8 \cdot 10^{-5}$ & 0.03 & 0.25 \\
BatchPU3 & DBTDL & 0.1 & $1 \cdot 10^{-3}$ & $4.8 \cdot 10^{-4}$ & 0.3 & 0.25 \\
BatchPU4 & DBTDL & 1.0 & $1 \cdot 10^{-2}$ & $4.8 \cdot 10^{-3}$ & 3.0 & 0.25
\end{tabular}




\begin{tabular}{lllllll} 
BatchPU5 & YIPO & 0.1 & $1 \cdot 10^{-3}$ & $4.8 \cdot 10^{-4}$ & 0.6 & 0.25 \\
BatchPU6 & DBU & 5.0 & 0.05 & $2.4 \cdot 10^{-2}$ & 3.6 & 0.25 \\
BatchPU7 & TBD & 5.0 & 0.05 & $2.4 \cdot 10^{-2}$ & 3.3 & 0.25 \\
\hline
\end{tabular}

Under a nitrogen atmosphere, $0.25 \mathrm{~mL}(0.48 \mathrm{mmol}, 1.00$ eq. $)$ of the MDI solution was combined with $1.0 \mathrm{~mL}(0.48 \mathrm{mmol}, 1.00$ eq. $)$ of the diol solution and $0.25 \mathrm{~mL}$ of the respective catalyst solution (or anhydrous THF for reaction under absence of catalyst).

The reaction mixture was stirred at room temperature and samples were taken after $1,2,5,10$ and 20 min followed by precipitated in petroleum ether. After removing solvents under reduced pressure, ${ }^{1} \mathrm{H}$ NMR spectra were recorded in DMSO- $d_{6}$ to investigate the reaction kinetics. Afterwards, the final sample (20 min reaction time) was purified twice by re-dissolving in THF and precipitating into $\mathrm{MeOH}$. For reactions catalyzed by $\mathrm{DBU}$ and $\mathrm{TBD}, \mathrm{MeOH}$ containing $4 \mathrm{v} / \mathrm{v}-\%$ TFA was used to improve catalyst removal. The precipitate was separated by centrifugation and dried overnight under vacuum at $40{ }^{\circ} \mathrm{C}$. The final products were characterized by FTIR, ${ }^{1} \mathrm{H}$ NMR and GPC.

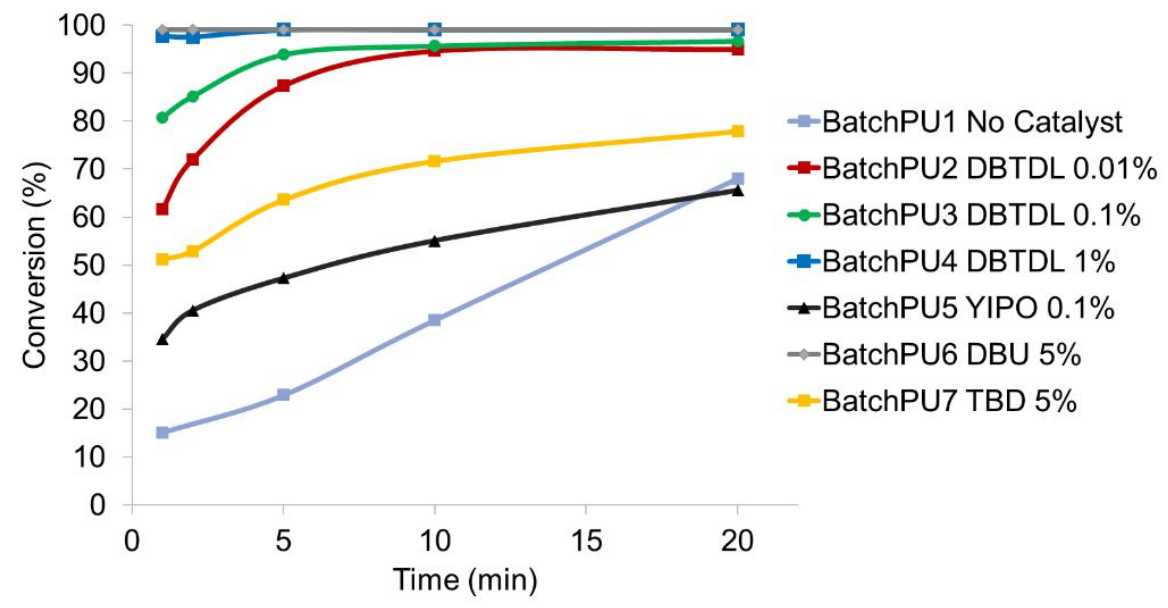

Figure S9: Effect of the catalyst on the monomer conversion for syntheses of PUs from MDI and PEG1500/HDO (40:60).

\subsubsection{Effect of varying the ratio of the diol monomers on reaction kinetics}




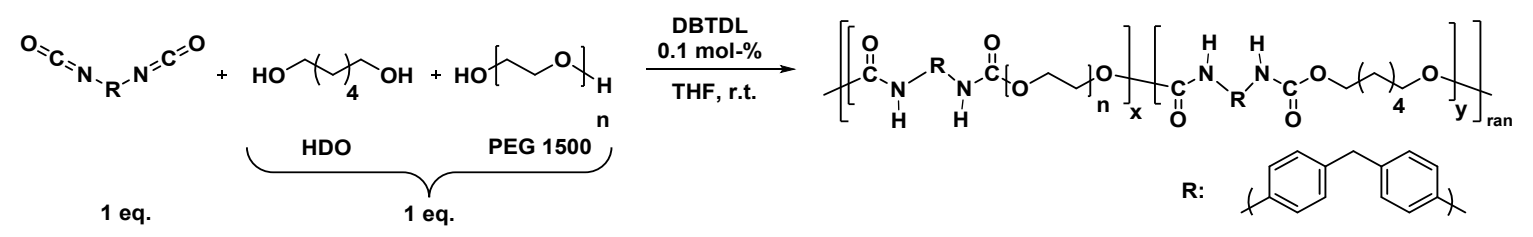

In a $\mathrm{N}_{2}$-filled glovebox, a $0.48 \mathrm{M}$ solution of PEG1500:HDO (50:50) was prepared by dissolving $1.47 \mathrm{~g}(0.96 \mathrm{mmol})$ of PEG1500 and $113 \mathrm{mg}(0.96 \mathrm{mmol})$ of HDO in anhydrous THF to a total volume of $4 \mathrm{~mL}$. Additionally, a $0.48 \mathrm{M}$ solution of HDO was prepared by dissolving $208 \mathrm{mg}$ (1.76 mmol) HDO in anhydrous THF to a total volume of $3.6 \mathrm{~mL}$, a $1.92 \mathrm{M}$ solution of MDI by dissolving $800 \mathrm{mg}(3.20 \mathrm{mmol}) \mathrm{MDI}$ in anhydrous THF to a total volume of $1.7 \mathrm{~mL}$, and a $1.9 \mathrm{mM}$ solution of the catalyst DBTDL by dissolving $2.0 \mathrm{mg}\left(3.2 \cdot 10^{-3} \mathrm{mmol}\right)$ in anhydrous THF to a total volume of $1.7 \mathrm{~mL}$.

Under nitrogen atmosphere, $0.25 \mathrm{~mL}$ of the MDI solution ( $0.48 \mathrm{mmol}, 1.0$ eq.) were combined in a vial with a mixture of the PEG1500:HDO and HDO solutions (total volume: $1.0 \mathrm{~mL}$, $0.48 \mathrm{mmol}, 1.0$ eq., individual volumes listed in Table S2). Subsequently, $0.25 \mathrm{~mL}$ of the DBTDL catalyst solution $\left(4.8 \cdot 10^{-4} \mathrm{~mol}, 0.001\right.$ eq. $)$ were added to the stirring mixture.

Samples were taken after 1,2, 5, 10 and $20 \mathrm{~min}$ and precipitated in petroleum ether. After removing the solvents under reduced pressure, ${ }^{1} \mathrm{H}$ NMR spectra were recorded in DMSO- $d_{6}$ to investigate the reaction kinetics. Afterwards, the final sample (20 min reaction time) was purified twice by re-dissolving in THF and precipitating into $\mathrm{MeOH}$. The solids were separated by centrifugation and dried overnight under vacuum at $40^{\circ} \mathrm{C}$. Protocols to prepare PUs with different weight fractions of the hard segment (HS) are shown in Table S2.

Table S2: Details about molar amounts, ratios and used solution volumes for syntheses of PUs with different percentages of hard and soft segments. 


\begin{tabular}{|c|c|c|c|c|c|c|c|}
\hline Sample & $\begin{array}{c}\text { MDI } \\
(\mathrm{mmol})\end{array}$ & $\begin{array}{c}\text { PEG1500 } \\
\text { (mmol) }\end{array}$ & $\begin{array}{c}\text { HDO } \\
(\mathrm{mmol})\end{array}$ & $\begin{array}{l}V_{\text {PEG1500:HDO }} \\
(\mathrm{mL})\end{array}$ & $\begin{array}{l}V_{\text {HDO }} \\
(\mathrm{mL})\end{array}$ & $\begin{array}{c}x_{H D O, t h} \\
(\%)\end{array}$ & $\begin{array}{c}w_{H S, t h} \\
(\%)\end{array}$ \\
\hline BatchPU8 & 0.48 & 0.24 & 0.24 & 1 & 0 & 50 & 29 \\
\hline BatchPU9 & 0.48 & 0.19 & 0.29 & 0.8 & 0.2 & 60 & 34 \\
\hline BatchPU10 & 0.48 & 0.14 & 0.34 & 0.6 & 0.4 & 70 & 42 \\
\hline BatchPU11 & 0.48 & 0.096 & 0.384 & 0.4 & 0.6 & 80 & 53 \\
\hline BatchPU12 & 0.48 & 0.048 & 0.432 & 0.2 & 0.8 & 90 & 70 \\
\hline
\end{tabular}

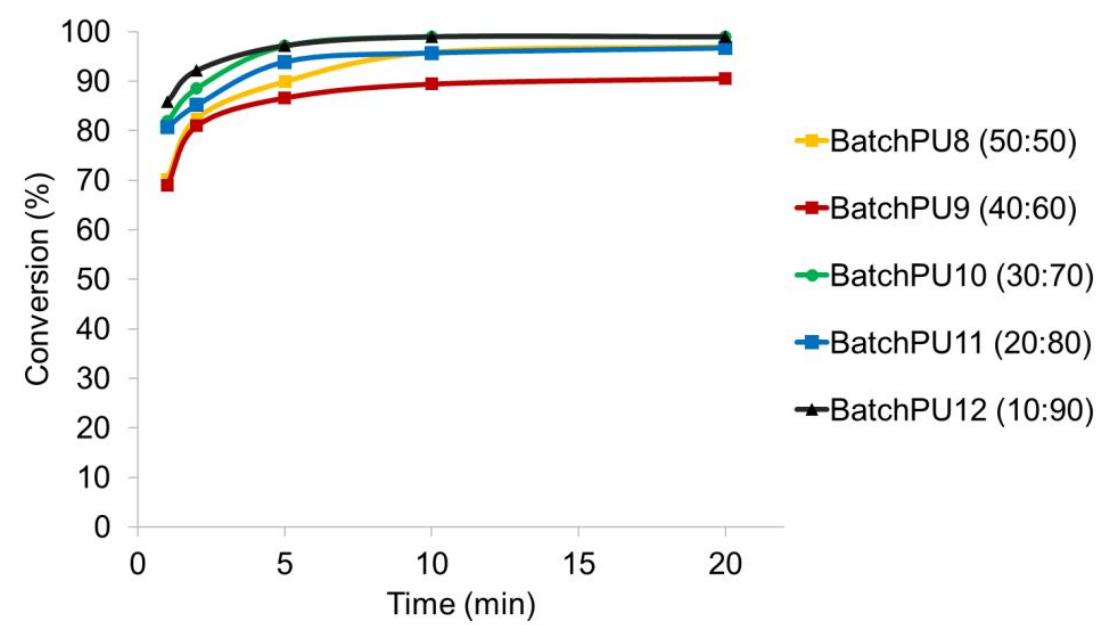

Figure S10: Monomer conversion over time for syntheses of PUs from MDI and PEG:HDO with different ratios

\subsubsection{Control of molecular weight by end-capping agent HEMA}




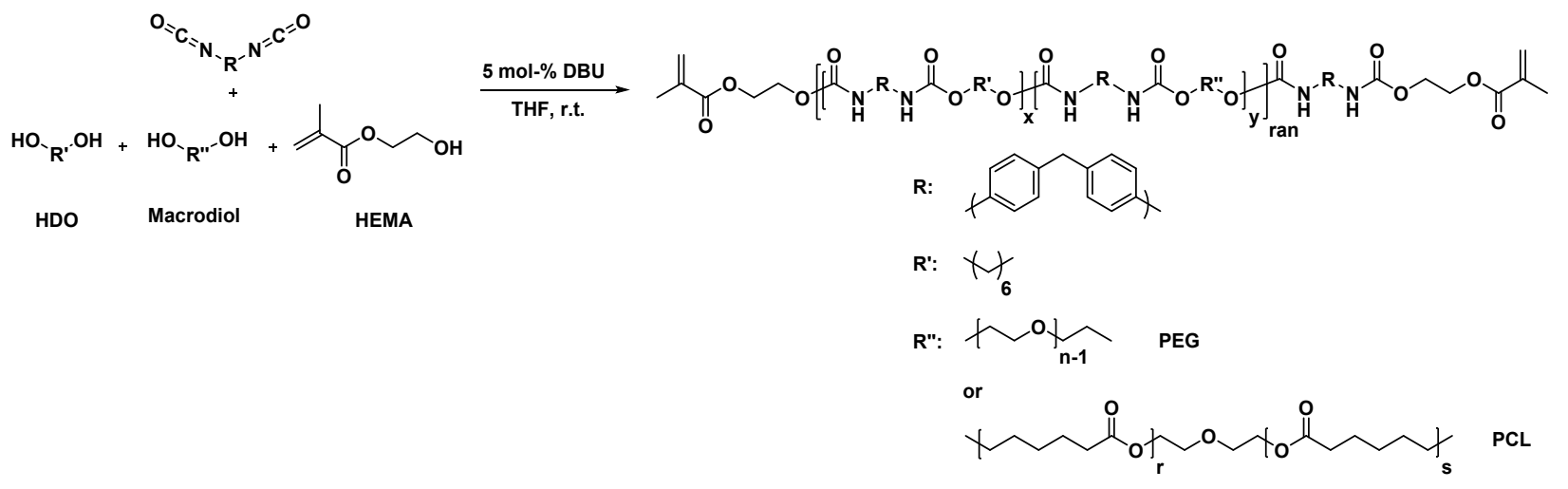

In a $\mathrm{N}_{2}$-filled glovebox a solution of PEG2000:HDO (40:60) and HEMA (molar ratio: 3.6:5.5:1) with a total of $1.92 \mathrm{mmol}$ hydroxyl groups of which 5 mol-\% were represented by HEMA was prepared by dissolving $731 \mathrm{mg}$ (0.36 mmol, 0.38 eq.) PEG2000, $65 \mathrm{mg}$ (0.55 mmol, 0.57 eq.) HDO and $13 \mathrm{mg}(0.10 \mathrm{mmol}, 0.1$ eq.) HEMA in anhydrous THF to a total volume of $2.1 \mathrm{~mL}$. A $1.92 \mathrm{M}$ MDI solution was prepared by dissolving $240 \mathrm{mg}$ (0.96 mmol, 1.00 eq.) MDI in anhydrous THF to a total volume of $0.5 \mathrm{~mL}$. A $9.6 \cdot 10^{-2} \mathrm{M} \mathrm{DBU}$ solution was prepared by dissolving $7.3 \mathrm{mg}\left(4.8 \cdot 10^{-2} \mathrm{mmol}, 0.05\right.$ eq. $)$ in $0.5 \mathrm{~mL}$ anhydrous THF.

The three solutions were combined $\left(\mathrm{c}_{\mathrm{MDI}}=0.32 \mathrm{M}\right)$ in a vial and stirred under nitrogen atmosphere. Samples were taken after $1 \mathrm{~min}$ and 10 min reaction time and precipitated into petroleum. After removing the solvents under reduced pressure, ${ }^{1} \mathrm{H}$ NMR spectra were recorded in DMSO- $d_{6}$ to investigate reaction kinetics. The final product (10 min reaction time) was purified twice by re-dissolving in THF and precipitating into $\mathrm{MeOH}$ containing $4 \mathrm{v} / \mathrm{v} \%$ TFA. Solids were separated by centrifugation and dried overnight in a vacuum oven at $40{ }^{\circ} \mathrm{C}$. The final product was characterized by ${ }^{1} \mathrm{H}$ NMR, FTIR and GPC.

\subsection{Syntheses and experiments using continuous flow reactors}




\subsubsection{Macrodiol PCL2800}

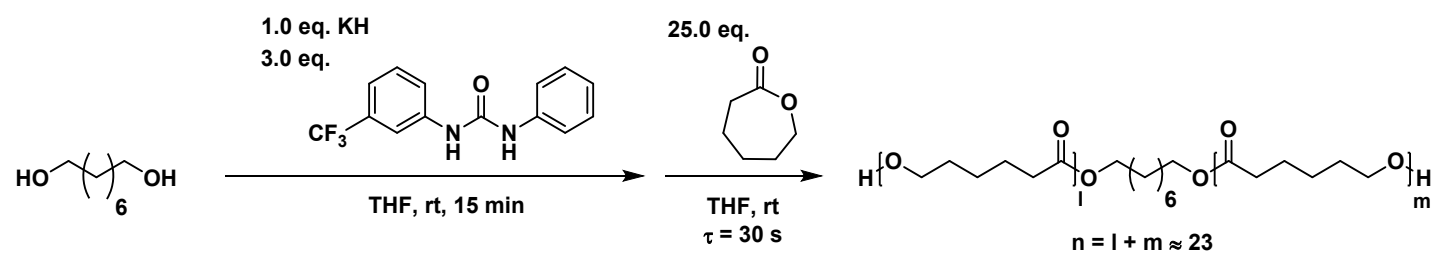

In a $\mathrm{N}_{2}$-filled glovebox, a $2.1 \mathrm{M}$ monomer solution was prepared by dissolving $12.00 \mathrm{~g}$ (105.13 mmol) $\varepsilon$-caprolactone in anhydrous THF up to a total volume of $50 \mathrm{~mL}$ and passing it through a $0.2 \mu \mathrm{m}$ PTFE filter. To prepare the catalyst/initiator solution, $0.60 \mathrm{~g}(4.10 \mathrm{mmol}$, 1.0 eq.) 1,8 -octanediol and $3.45 \mathrm{~g}$ (12.32 mmol, 3.0 eq.) $\mathrm{CF}_{3} \mathrm{PhUPh}$ were dissolved in $20 \mathrm{~mL}$ anhydrous THF and combined with a suspension of $0.33 \mathrm{~g}(8.21 \mathrm{mmol}, 2.0$ eq.) potassium hydride in $20 \mathrm{~mL}$ anhydrous THF. Stirring of this mixture was stopped 15 min after the gas formation had ceased. The mixture was diluted with anhydrous THF to $54 \mathrm{~mL}$ and passed through a $0.2 \mu \mathrm{m}$ PTFE filter. The quenching solution was prepared by dissolving $6.01 \mathrm{~g}$ (49.24 mmol, 12.0 eq.) benzoic acid in $40 \mathrm{~mL}$ of THF.

After flushing the flow reactor system (Figure S11) with anhydrous THF (10 mL), syringes with the monomer and initiator/catalyst were connected and the reactor was run for 46 min at a total flow rate of $2.20 \mathrm{~mL} / \mathrm{min}$ resulting in a residence time of $30 \mathrm{~s}$ (see parameters in Table S3). The crude polymer solution was concentrated under a stream of air and the crude material was precipitated into methanol. The solid was separated by centrifugation and the supernatant decanted. This process of precipitation, centrifugation, and decantation was performed four additional times. Finally, water was removed from the isolated material by three successive azeotropic distillations under reduced pressure using anhydrous toluene.

Conversion: $88 \%$. Yield: $9.17 \mathrm{~g}$ (76\%, based on conversion). ${ }^{1} \mathrm{H}$ NMR (400 MHz, DMSO- $d_{6}$, $\delta):=4.35(2 \mathrm{H}), 3.98(47 \mathrm{H}), 3.35(4 \mathrm{H}), 2.27(47 \mathrm{H}, \mathrm{d}), 1.75-1.03(157 \mathrm{H}) . \mathrm{GPC}\left(\mathrm{THF}, 25{ }^{\circ} \mathrm{C}\right):$ $\mathrm{M}_{\mathrm{n}}=4.11 \mathrm{~kg} / \mathrm{mol}, \mathrm{M}_{\mathrm{w}}=5.36 \mathrm{~kg} / \mathrm{mol}, \mathrm{Ð}=1.30 . \mathrm{DP}_{\mathrm{NMR}}=23.5 . \mathrm{M}_{\mathrm{n}, \mathrm{NMR}}=2824 \mathrm{~g} / \mathrm{mol}$. 

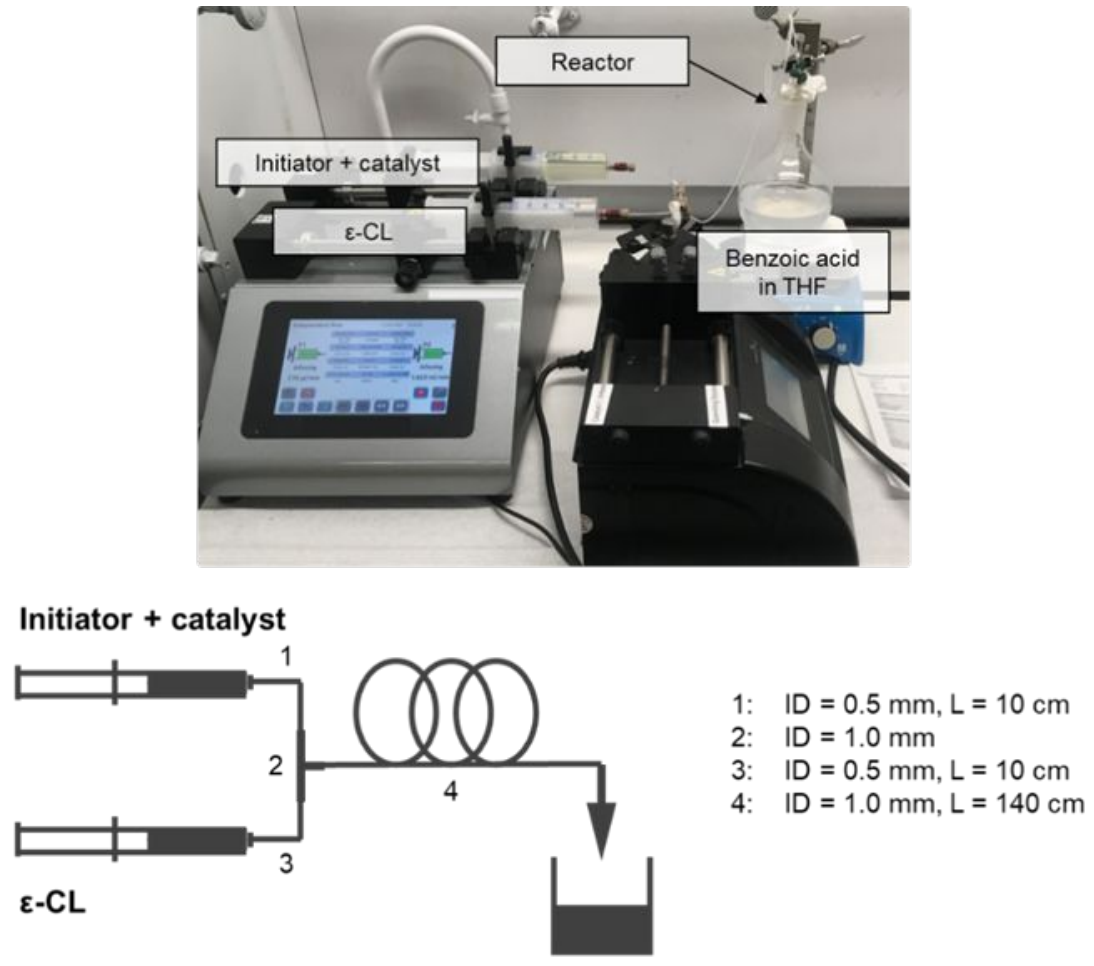

Benzoic acid in THF

Figure S11: Flow reactor setup for synthesizing macrodiol PCL2800.

Table S3: Flow reaction parameters for synthesizing macrodiol PCL2800.

\begin{tabular}{lccc}
\hline & \multicolumn{2}{c}{ Flow rate } & \\
& $(\mathbf{m L} / \mathbf{m i n})$ & $(\mathbf{m m o l} / \mathbf{m i n})$ & Molar equivalents \\
\hline Monomer & 1.044 & 2.195 & 25 \\
Initiator/Catalyst & 1.155 & 0.088 & 1 \\
\hline
\end{tabular}

\subsubsection{Catalytic performance of DBTDL and DBU}




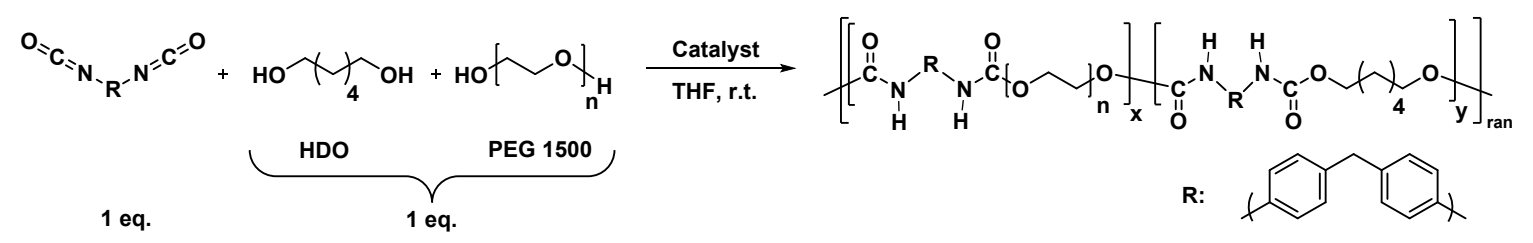

Three different catalyst solutions were investigated for the polymerization of PEG1500:HDO and MDI using flow reactors.

For each polymerization the monomer solutions were prepared as follows: In a $\mathrm{N}_{2}$-filled glovebox a 0.48 M solution of PEG1500:HDO (40:60) solution was prepared by dissolving $2243 \mathrm{mg}$ ( $1.46 \mathrm{mmol}, 0.40$ eq.) PEG1500 and $259 \mathrm{mg}$ (2.19 mmol, 0.60 eq.) HDO in anhydrous THF to a total volume of $7.6 \mathrm{~mL}$. A $1.92 \mathrm{M}$ MDI solution was prepared by dissolving $913 \mathrm{mg}$ (3.65 mmol, 1.00 eq.) of MDI in anhydrous THF to a total volume of $1.9 \mathrm{~mL}$. For the reaction catalyzed by 1.0 mol-\% DBTDL, a $19.2 \mathrm{mM}$ DBTDL solution was prepared by dissolving $23 \mathrm{mg}\left(3.65 \cdot 10^{-2} \mathrm{mmol}, 0.01\right.$ eq. $)$ DBTDL in anhydrous THF to a total volume of $1.9 \mathrm{~mL}$. For the reaction catalyzed by $0.1 \mathrm{~mol}-\%$ DBTDL, a solution of $1.92 \mathrm{mM}$ DBTDL was prepared by dissolving $2.0 \mathrm{mg}\left(3.65 \cdot 10^{-3} \mathrm{mmol}, 1 \cdot 10^{-3}\right.$ eq. $)$ DBTDL in $1.9 \mathrm{~mL}$ anhydrous THF. Accordingly, for the reaction catalyzed by 5 mol-\% DBU a solution of $0.096 \mathrm{M}$ DBU was prepared by dissolving $28 \mathrm{mg}(0.182 \mathrm{mmol}, 0.05$ eq. $)$ DBU in $1.9 \mathrm{~mL}$ of anhydrous THF. For conducting the polyaddition reaction a flow reactor setup was used as shown in Figure S12. The reactor system was flushed with anhydrous THF $(10 \mathrm{~mL})$ prior to connecting the syringes holding the stock solutions. The residence time was modified by changing the flow rate of each syringe, albeit a 4:1:1 flow ratio between PEG1500/HDO:MDI:catalyst solutions was maintained to keep the stoichiometry constant (Table $\mathbf{S 4}$ ). 


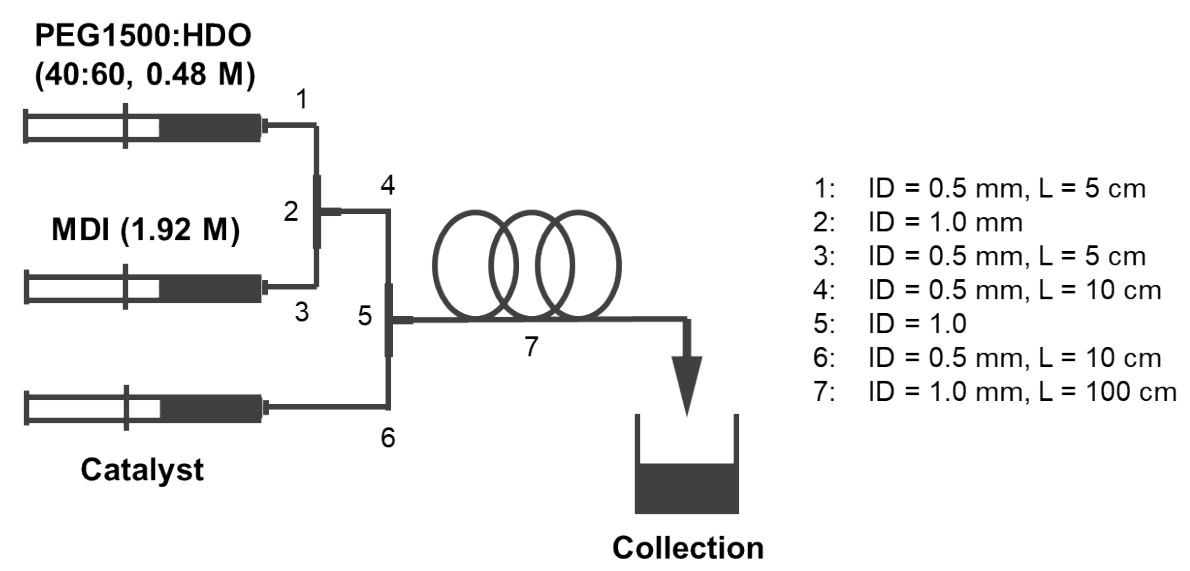

Figure S12: Reactor setup for the catalyst screening in the flow-system.

Table S4: Residence time and flow rates for the polymerization of PEG1500:HDO (40:60) and MDI catalyzed by 1 mol-\% DBTDL, 0.1 mol-\% DBTDL or 5 mol-\% DBU.

\begin{tabular}{lcccc}
\hline $\boldsymbol{\tau}(\mathbf{s})$ & Total & \multicolumn{2}{c}{ Total flow rate( $\boldsymbol{\mu L} / \mathbf{m i n})$} & \\
& MDI & Catalyst & PEG1500:HDO \\
\hline 30 & 1570.8 & 261.8 & 261.8 & 1047.2 \\
60 & 785.4 & 130.9 & 130.9 & 523.6 \\
120 & 392.3 & 65.4 & 65.4 & 261.8 \\
180 & 261.8 & 43.6 & 43.6 & 174.5 \\
240 & 196.3 & 32.7 & 32.7 & 130.9 \\
300 & 157.1 & 26.2 & 26.2 & 104.7 \\
\hline
\end{tabular}

Before each run the flow reactor was flushed with anhydrous THF $(10 \mathrm{ml})$ and, then, run 1.5fold the residence time to allow for a steady state before collecting the product fractions by direct precipitation into petroleum ether. Solvents were removed under reduced pressure and the crude material re-dissolved in DMSO- $d_{6}$ for ${ }^{1} \mathrm{H}$ NMR analysis. Afterwards, the crude materials were purified twice by re-dissolving in THF and precipitating into $\mathrm{MeOH}$. When using DBU as catalyst, $\mathrm{MeOH}$ with 4 vol-\% TFA was used for the precipitations to improve 
catalyst removal. The obtained white solids were isolated by centrifugation, dried under reduced pressure, and analyzed by ${ }^{1} \mathrm{H}$ NMR, FTIR and GPC.
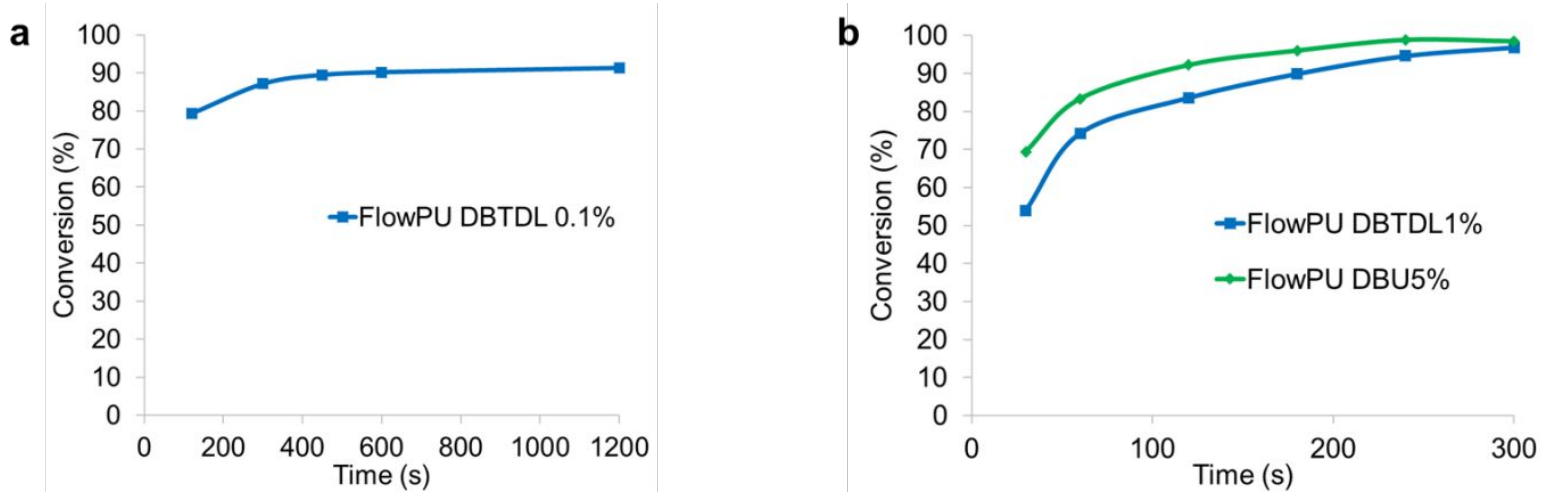

Figure S13: Conversion over time for continuous-flow polymerizations of MDI and PEG1500:HDO (40:60) catalyzed by 0.1 mol-\% DBTDL (a) and 1 mol-\% DBTDL (b, blue) and 5 mol-\% DBU (b, green).

FlowPU DBTDL 1\%: $\quad \tau=5 \mathrm{~min} \quad \mathrm{M}_{\mathrm{n}}=33.5 \mathrm{~kg} / \mathrm{mol}, \mathrm{Ð}=1.2$

FlowPU DBTDL 0.1\%: $\quad \tau=20 \mathrm{~min} \quad \mathrm{M}_{\mathrm{n}}=31.8 \mathrm{~kg} / \mathrm{mol}, \mathrm{Ð}=1.3$

FlowPU DBU 5\%: $\quad \tau=5 \mathrm{~min} \quad \mathrm{M}_{\mathrm{n}}=37.9 \mathrm{~kg} / \mathrm{mol}, \mathrm{Ð}=1.2$

\subsubsection{In-line ATR-FTIR calibration}

In a $\mathrm{N}_{2}$-filled glovebox, a $0.32 \mathrm{M}$ solution of MDI was prepared by dissolving $80.1 \mathrm{mg}$ $(0.32 \mathrm{mmol})$ of MDI in anhydrous THF to a total volume of $1 \mathrm{~mL}$. An additional syringe was filled with $2 \mathrm{~mL}$ anhydrous THF. The syringes were connected to a continuous-flow reactor similar to Figure S11 but including an in-line ATR-FTIR flow cell Figure S14. 


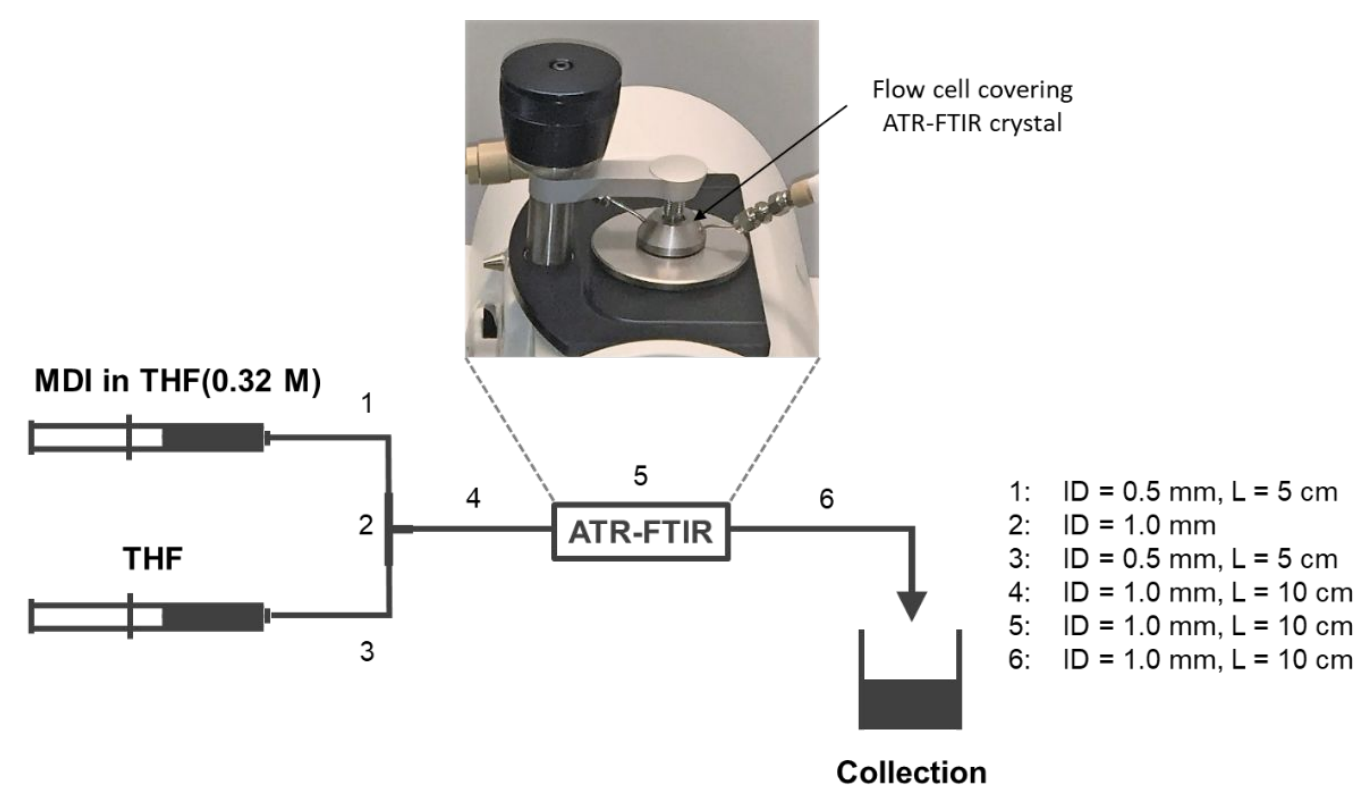

Figure S14: Reactor setup for FTIR calibration experiment.

Flow rates of the MDI and anhydrous THF solutions were changed every 3 min keeping a total flow rate of $36 \mu \mathrm{L} / \mathrm{min}$, and thus, simulating the consumption of isocyanate group over time (Table S5). FTIR spectra were recorded every 20 seconds.

Table S5: Flow rates, calculated MDI concentration and related conversion for in-line FTIR calibration experiment.

\begin{tabular}{|c|c|c|c|c|}
\hline \multicolumn{3}{|c|}{ Flow rate $(\mu \mathrm{L} / \mathrm{min})$} & \multirow{2}{*}{$\begin{array}{c}\mathbf{c}_{\text {MDI,Reactor }} \\
(\mathrm{mol} / \mathrm{L})\end{array}$} & \multirow{2}{*}{$\begin{array}{c}\text { Related conversion } \\
(\%)\end{array}$} \\
\hline Total & MDI & THF & & \\
\hline 36 & 36 & 0 & 0.32 & 0 \\
\hline 36 & 32.4 & 3.6 & 0.288 & 10 \\
\hline 36 & 28.8 & 7.2 & 0.256 & 20 \\
\hline 36 & 25.2 & 10.8 & 0.224 & 30 \\
\hline 36 & 21.6 & 14.4 & 0.192 & 40 \\
\hline 36 & 18.0 & 18.0 & 0.16 & 50 \\
\hline 36 & 14.4 & 21.6 & 0.128 & 60 \\
\hline 36 & 10.8 & 25.2 & 0.096 & 70 \\
\hline 36 & 7.2 & 28.8 & 0.064 & 80 \\
\hline 36 & 3.6 & 32.4 & 0.032 & 90 \\
\hline 36 & 0 & 36 & 0 & 100 \\
\hline
\end{tabular}


a)

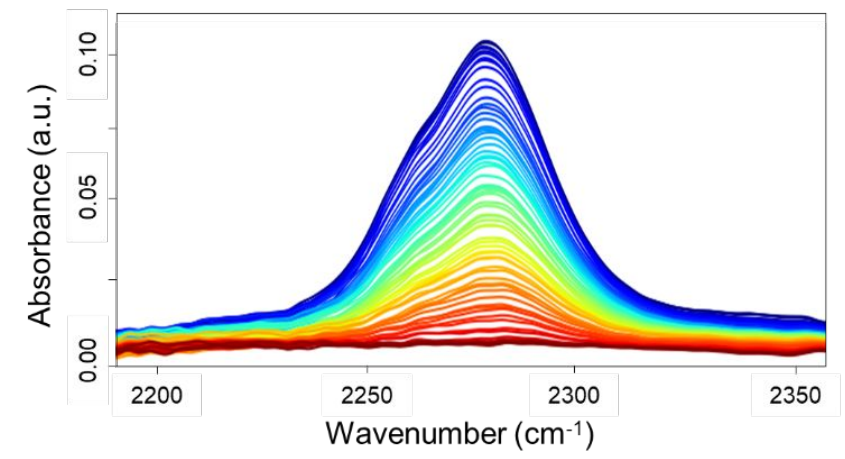

b)

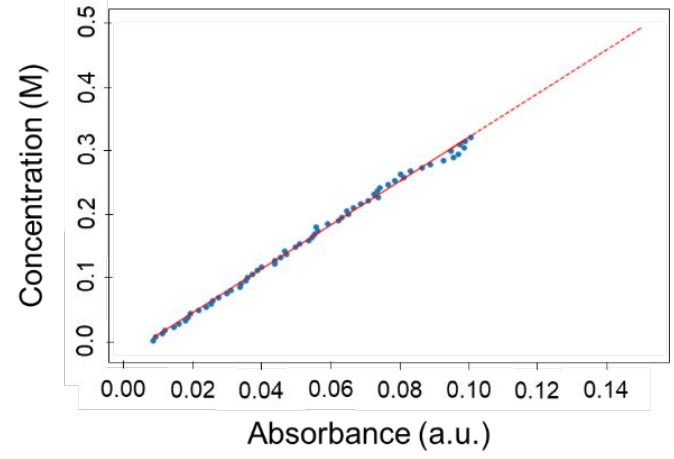

Figure S15: Section of the FTIR spectra showing the decrease in absorbance of the characteristic NCO band at $2270 \mathrm{~cm}^{-1}$ with decrease in concentration (a, blue to red). Plot showing the dependence between absorbance at $2270 \mathrm{~cm}^{-1}$ and concentration of MDI (b).

The experimental data showed a very high correlation between concentration of MDI $c_{M D I}$ and the absorbance at $2270 \mathrm{~cm}^{-1} A b_{2270}$ and linear regression returned the following expression numerically describing the dependence:

$$
c_{M D I}=-0.0264+3.4615 \times A b s_{2270}
$$

This expression was further used to calculate the monomer conversion in real-time when inline reaction monitoring was applied for polymerizations in continuous flow reactors and found application in the custom developed process control software real-time capable of programmatically controlling syringe pumps and ATR-FTIR, spectra processing and visualization, and conversion calculation.

\subsubsection{Synthesis of PUs with varying amount of HS}

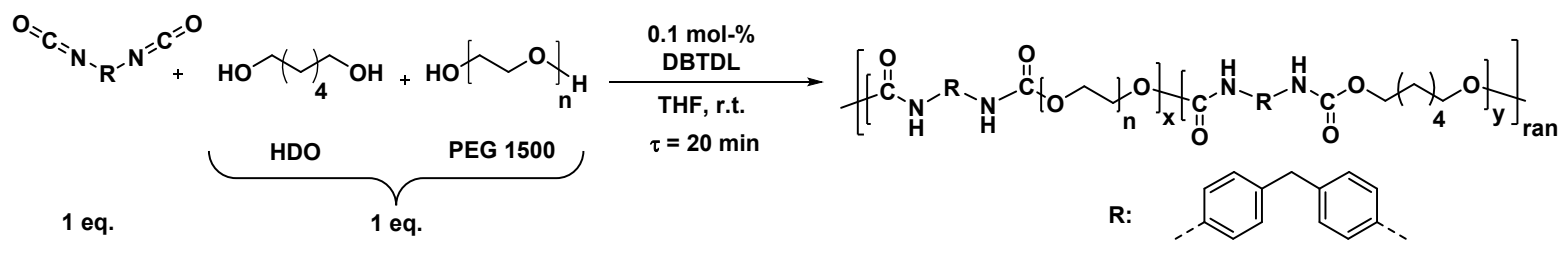


In a $\mathrm{N}_{2}$-filled glovebox a $0.48 \mathrm{M}$ solution of PEG1500:HDO (50:50) was prepared in anhydrous THF by dissolving $4.92 \mathrm{~g}$ (3.20 mmol, 0.50 eq.) PEG1500 and $378 \mathrm{mg}$ (3.20 mmol, 0.50 eq.) HDO to a total volume of $13.3 \mathrm{~mL}$ and a $0.48 \mathrm{M}$ solution of HDO by dissolving $378 \mathrm{mg}$ (3.20 mmol, 0.50 eq.) HDO in anhydrous THF to a total volume of $6.6 \mathrm{~mL}$. A $1.92 \mathrm{M}$ MDI solution was prepared by dissolving $1.60 \mathrm{~g}$ (6.40 mmol, 1 eq.) MDI in anhydrous THF to a total volume of $3.3 \mathrm{~mL}$. A $19.2 \mathrm{mM}$ DBTDL solution was prepared by dissolving $4.0 \mathrm{mg}$ $\left(6.4 \cdot 10^{-3} \mathrm{mmol}, 1 \cdot 10^{-3}\right.$ eq.) DBTDL in $3.2 \mathrm{~mL}$ anhydrous THF. After flushing the flow reactor with anhydrous THF the syringes holding the prepared solutions were connected to the reactor (Figure S16).

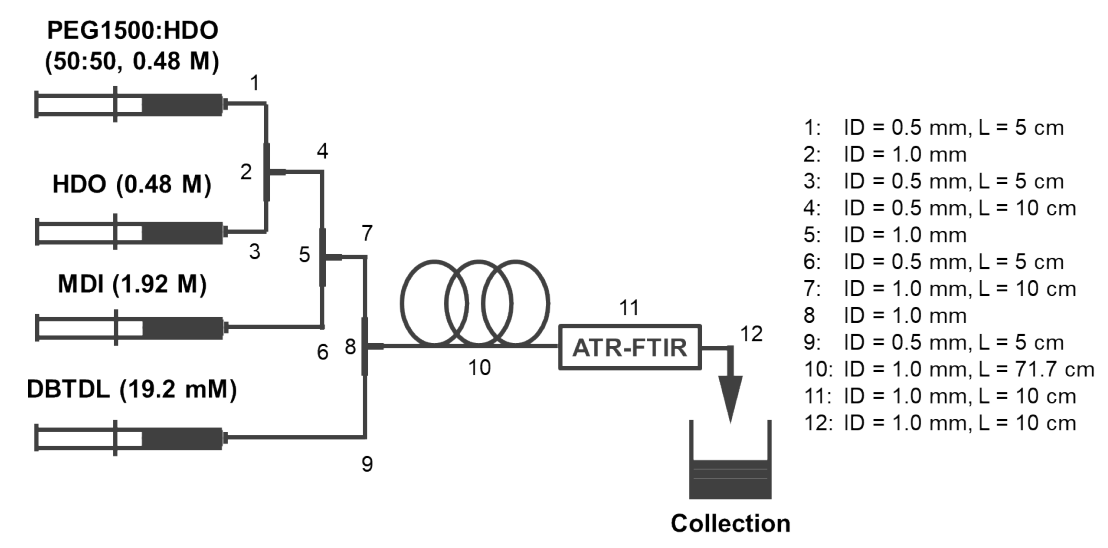

Figure S16: Reactor setup for the straightforward on-demand synthesis of PUs with variable contents of HS.

The residence time in the reactor was set to $20 \mathrm{~min}$ corresponding to a total flow rate of $36 \mu \mathrm{L} / \mathrm{min}$. The molar feed ratio between macrodiol PEG1500 and HDO was periodically modified by varying the flow rates for the PEG1500:HDO (50:50) mixture and the HDO solution (Table S6). 
Table S6: Reaction specifications for the tunable HS \% PU synthesis.

\begin{tabular}{|c|c|c|c|c|c|c|c|c|}
\hline \multirow{2}{*}{ Entry } & \multicolumn{2}{|c|}{$\begin{array}{c}\text { Molar flow rate } \\
(\mu \mathrm{mol} / \mathrm{min})\end{array}$} & \multirow[b]{2}{*}{ MDI } & \multicolumn{3}{|c|}{ Flow rate $(\mu \mathrm{L} / \mathrm{min})$} & \multirow{2}{*}{$\begin{array}{c}\text { Molar ratio } \\
\text { PEG1500:HDO }\end{array}$} & \multirow{2}{*}{$\begin{array}{l}\text { HS } \\
(\%)\end{array}$} \\
\hline & MDI & Diols $_{\text {total }}$ & & Diols $_{\text {total }}$ & HDO & PEG1500:HDO & & \\
\hline FlowPU1 & 11.5 & 11.5 & 6 & 24 & 2.4 & 21.6 & $45: 55$ & 31.2 \\
\hline FlowPU2 & 11.5 & 11.5 & 6 & 24 & 7.2 & 16.8 & $35: 65$ & 37.8 \\
\hline FlowPU3 & 11.5 & 11.5 & 6 & 24 & 12 & 12 & $25: 75$ & 46.9 \\
\hline FlowPU4 & 11.5 & 11.5 & 6 & 24 & 16.8 & 7.2 & $15: 85$ & 60.3 \\
\hline FlowPU5 & 11.5 & 11.5 & 6 & 24 & 21.6 & 2.4 & $5: 95$ & 82.5 \\
\hline
\end{tabular}

It must be noted that although an increase of the viscosity of the output was observed over time, no clogging occurred during the polymerization process. Monomer conversion was programmatically determined in real-time every $20 \mathrm{~s}$ by connecting an in-line ATR-FTIR via flow cell and applying Equation S14 (implemented in custom software). The product fractions were collected once the conversion reached stable values above $90 \%$ and directly precipitated into petroleum ether, filtered, re-dissolved in THF and precipitated in $\mathrm{MeOH}$. After repeating the purification by precipitation two more times, solvents were removed under reduced pressure and the products characterized by FTIR, ${ }^{1} \mathrm{H}$ NMR, GPC, TGA and DSC.

Table S7: Experimental details for FlowPU1 to FlowPU5.

\begin{tabular}{lccccc}
\hline Entry & $\begin{array}{c}\text { Conversion } \\
(\%)^{\mathbf{a}}\end{array}$ & $\begin{array}{c}\boldsymbol{x}_{\text {HDo }} \\
(\mathbf{\%})^{\mathbf{a}}\end{array}$ & $\begin{array}{c}\mathbf{M}_{\mathbf{n}} \\
(\mathbf{k g} / \mathbf{m o l})^{\mathbf{b}}\end{array}$ & $\mathbf{\Xi}^{\mathbf{b}}$ & $\begin{array}{c}\mathbf{T}_{\mathbf{g}} \\
\left({ }^{\mathbf{C}} \mathbf{C}\right)^{\mathbf{c}}\end{array}$ \\
\hline FlowPU1 & 90 & 50 & 22.0 & 1.43 & -38 \\
FlowPU2 & 94 & 60 & 29.0 & 1.45 & -35 \\
FlowPU3 & 95 & 73 & 29.8 & 1.55 & -36 \\
FlowPU4 & 98 & 81 & 29.0 & 1.56 & -24 \\
FlowPU5 & 98 & 91 & 27.3 & 1.68 & -19 \\
\hline${ }^{a}$ Determined by ${ }^{1} \mathrm{H}$ NMR, ${ }^{\mathrm{b}}$ Determined by GPC vs PS standards. ${ }^{\mathrm{c}}$ Determined by DSC.
\end{tabular}




\subsubsection{Small-scale PU synthesis with end-capping by HEMA}

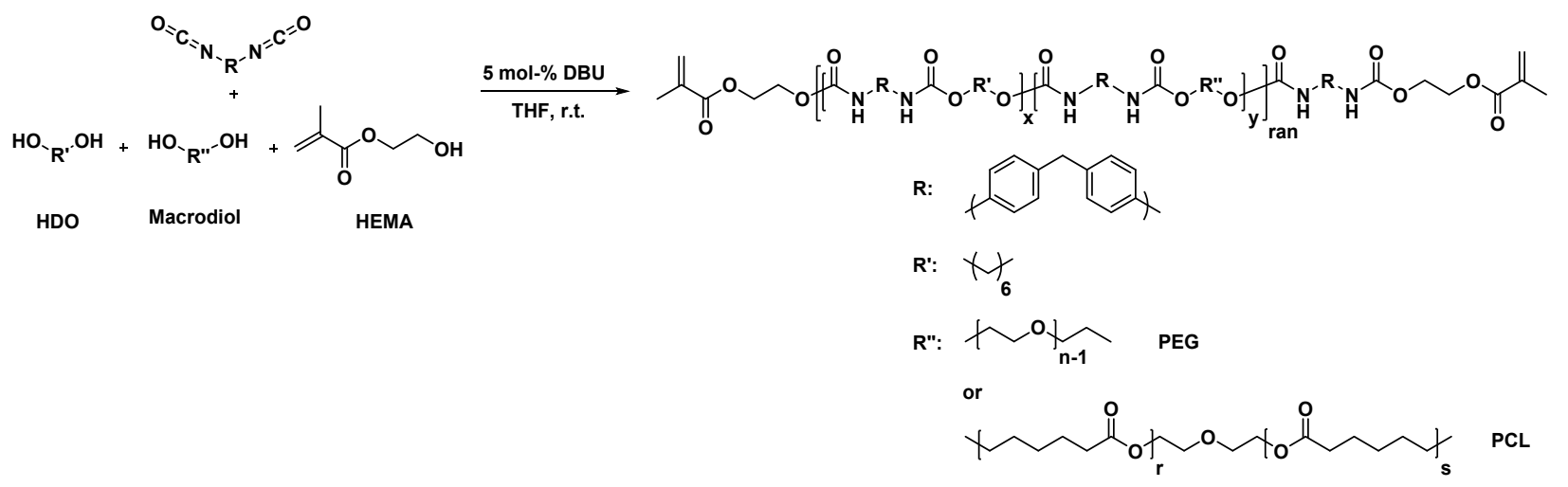

In a $\mathrm{N}_{2}$-filled glovebox a solution of PEG2000:HDO or PCL2000:HDO (40:60) and HEMA with a total of 4.99 mmol hydroxyl groups of which 5 mol-\% were represented by HEMA was prepared by dissolving $1.91 \mathrm{~g}$ (0.95 mmol, 0.38 eq.) PEG2000 or $2156 \mathrm{mg}$ (0.95 mmol, 0.38 eq.) of PCL2000, $168 \mathrm{mg}$ (1.42 mmol, 0.57 eq.) HDO and $33 \mathrm{mg}$ (0.25 mmol, 0.10 eq.) HEMA in anhydrous THF to a total volume of $5.5 \mathrm{~mL}$.

A 1.92 M MDI solution was prepared by dissolving $625 \mathrm{mg}$ (2.50 mmol, 1.00 eq. $)$ of MDI in anhydrous THF to a total volume of $1.3 \mathrm{~mL}$. A $9.6 \cdot 10^{-2} \mathrm{M}$ DBU solution was prepared by dissolving $19.0 \mathrm{mg}(0.13 \mathrm{mmol}, 0.05$ eq.) in $1.3 \mathrm{~mL}$ of anhydrous THF. The solutions were loaded into syringes and connected to the flow reactor after it was purged with anhydrous THF. (Figure S17). The retention times were set to $120 \mathrm{~s}, 180 \mathrm{~s}, 240 \mathrm{~s}$, and $300 \mathrm{~s}$ by varying the flow rate of each component (Table S8). 


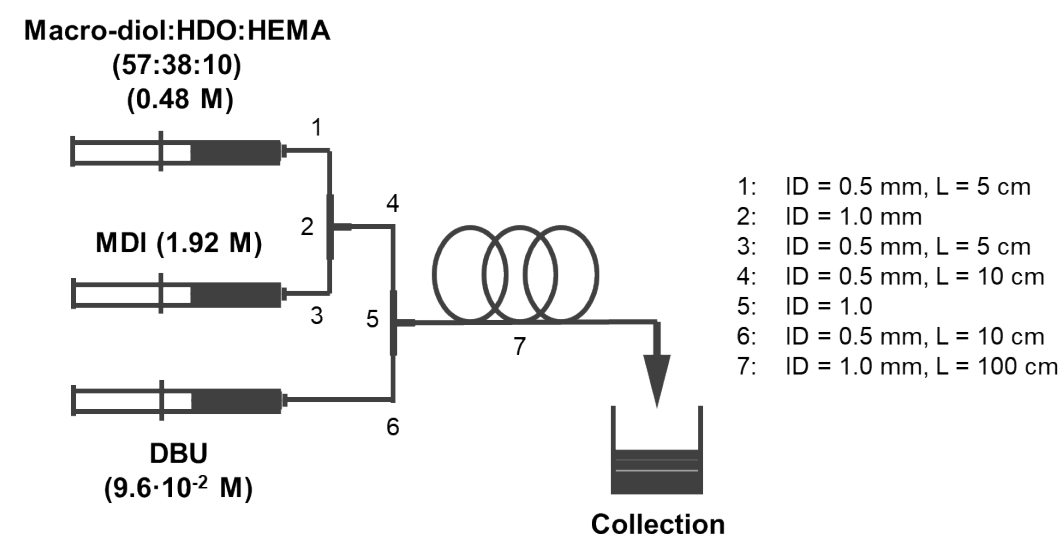

Figure S17: Flow reactor setup for PU syntheses including end-capping with HEMA.

Table S8: Retention time and flow rates for the small-scale preparation of PUs under control of molecular weight by end-capping with HEMA.

\begin{tabular}{ccccccc}
\hline$\tau(\mathbf{s})$ & $\begin{array}{c}\text { Total } \\
(\boldsymbol{\mu L} / \mathbf{m i n})\end{array}$ & $\begin{array}{c}\text { MDI } \\
(\boldsymbol{\mu L} / \mathbf{m i n})\end{array}$ & $\begin{array}{c}\text { DBU } \\
(\boldsymbol{\mu L} / \mathbf{m i n})\end{array}$ & $\begin{array}{c}\text { Macro- } \\
\text { diol:HDO:HEMA } \\
(\boldsymbol{\mu} \mathbf{L} / \mathbf{m i n})\end{array}$ & $\begin{array}{c}\text { NCO groups } \\
(\mathbf{m m o l} / \mathbf{m i n})\end{array}$ & $\begin{array}{c}\text { OH groups } \\
(\mathbf{m m o l} / \mathbf{m i n})\end{array}$ \\
\hline 120 & 392.3 & 65.4 & 65.4 & 261.8 & $1.3 \cdot 10^{-1}$ & $1.3 \cdot 10^{-1}$ \\
180 & 261.8 & 43.6 & 43.6 & 174.5 & $8.410^{-2}$ & $8.4 \cdot 10^{-2}$ \\
240 & 196.3 & 32.7 & 32.7 & 130.9 & $6.3 \cdot 10^{-2}$ & $6.3 \cdot 10^{-2}$ \\
300 & 157.1 & 26.2 & 26.2 & 104.7 & $5.0 \cdot 10^{-2}$ & $5.0 \cdot 10^{-2}$ \\
\hline
\end{tabular}

To ensure a steady-state in the reactor, the experiment was run for 1.5 -fold the residence time before collecting product fractions by direct precipitation into petroleum ether. After every run, the flow reactor was purged with anhydrous THF. Solvents from the crude samples were removed under reduced pressure and the solids analyzed by ${ }^{1} \mathrm{H}$ NMR and GPC. The samples prepared at a residence time of $300 \mathrm{~s}$ were purified twice by re-dissolving in THF and precipitation into $\mathrm{MeOH}$ containing 4 vol-\% TFA. After filtration and drying under reduced pressure, the product was obtained as white solid and further characterized by ${ }^{1} \mathrm{H}$ NMR, FTIR and GPC. 


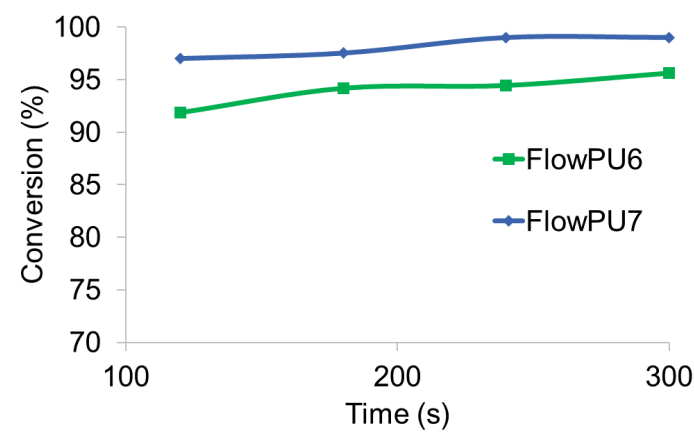

Figure S18: Monomer conversion over residence time for the continuous-flow synthesis of PUs FlowPU6 and FlowPU7 using PEG2000 (green) or PCL2000 as macrodiol (blue), respectively.

\subsubsection{Large-scale PU synthesis with end-capping by HEMA}

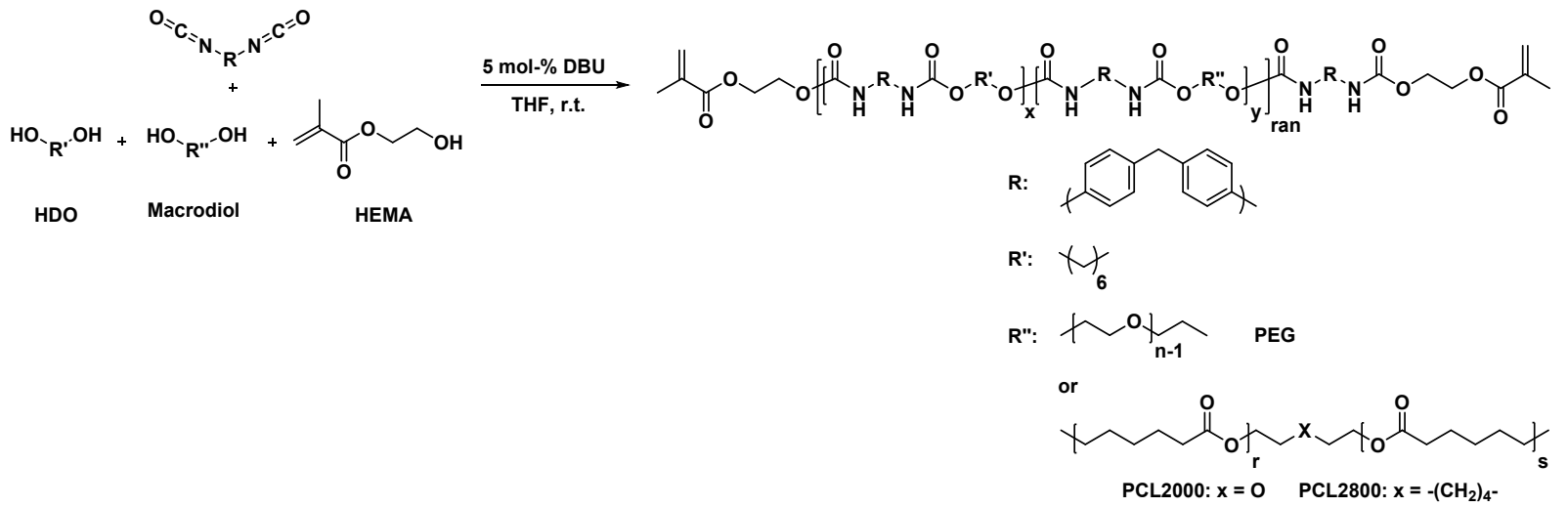

In a $\mathrm{N}_{2}$-filled glovebox a solution of macrodiol, HDO and HEMA (molar ratio for $x_{H D O, t h}=$ $60 \%$ : 3.8:5.7:1.0; molar ratio for $\left.x_{H D O, t h}=80 \%: 1.9: 7.6: 1.0\right)$ with HEMA taking up 5 mol- $\%$ of the total alcohol groups was prepared by following Table S9 and dissolving all components in anhydrous THF. A $1.92 \mathrm{M}$ solution of MDI and a solution of 5 mol-\% DBU were prepared similarly. All required information for each entry is listed in Table S9. The solutions were loaded into syringes and connected to a previously with anhydrous THF purged flow reactor (Figure S17). 
Table S9: Details about reaction entries for the preparation of HEMA-endcapped PUs at larger scales: choice of macrodiol, solution compositions, theoretical fraction of HDO, residence time.

\begin{tabular}{|c|c|c|c|c|c|c|c|c|}
\hline \multirow{3}{*}{ Entry } & \multirow{3}{*}{$\begin{array}{l}\text { Macro- } \\
\text { diol }\end{array}$} & \multicolumn{5}{|c|}{$\begin{array}{c}\text { Composition of MDI, DBU and Diol solutions } \\
\text { m [mg], } \mathbf{n}[\mathrm{mmol}], \mathrm{V}_{\text {total }}[\mathrm{mL}]^{\mathrm{a}}\end{array}$} & \multirow{3}{*}{$x_{H D O, t h}$} & \multirow{3}{*}{$\tau(\mathbf{s})$} \\
\hline & & \multirow{2}{*}{ MDI } & \multirow{2}{*}{ DBU } & \multicolumn{3}{|c|}{ Diol } & & \\
\hline & & & & HEMA & Macrodiol & HDO & & \\
\hline \multirow[t]{2}{*}{ FlowPU8 } & \multirow[t]{2}{*}{ PCL2000 } & \multirow{2}{*}{$\begin{array}{c}3400 \\
13.6 \\
7.0\end{array}$} & \multirow{2}{*}{$\begin{array}{c}103 \\
0.68 \\
7.0\end{array}$} & $\begin{array}{l}177 \\
1.36\end{array}$ & $\begin{array}{l}11730 \\
5.16\end{array}$ & $\begin{array}{l}915 \\
7.74\end{array}$ & \multirow[t]{2}{*}{60} & \multirow[t]{2}{*}{210} \\
\hline & & & & & $\mathrm{V}_{\text {total }}=28.3$ & & & \\
\hline \multirow[t]{2}{*}{ FlowPU9 } & \multirow[t]{2}{*}{ PCL2000 } & \multirow{2}{*}{$\begin{array}{c}4800 \\
19.2 \\
10.0\end{array}$} & \multirow{2}{*}{$\begin{array}{c}146 \\
0.96 \\
10.0\end{array}$} & $\begin{array}{l}130 \\
1.92\end{array}$ & $\begin{array}{l}8280 \\
3.64\end{array}$ & $\begin{array}{l}1723 \\
14.6\end{array}$ & \multirow[t]{2}{*}{80} & \multirow[t]{2}{*}{210} \\
\hline & & & & & $\mathrm{V}_{\text {total }}=42.0$ & & & \\
\hline \multirow[t]{2}{*}{ FlowPU10 } & \multirow[t]{2}{*}{ PCL2800 } & \multirow{2}{*}{$\begin{array}{c}2115 \\
8.45 \\
4.4\end{array}$} & \multirow{2}{*}{$\begin{array}{c}64 \\
0.42 \\
4.04\end{array}$} & $\begin{array}{l}110 \\
0.85\end{array}$ & $\begin{array}{l}9073 \\
3.21\end{array}$ & $\begin{array}{l}569 \\
4.82\end{array}$ & \multirow[t]{2}{*}{60} & \multirow[t]{2}{*}{240} \\
\hline & & & & & $\mathrm{V}_{\text {total }}=17.6$ & & & \\
\hline \multirow[t]{2}{*}{ FlowPU11 } & \multirow[t]{2}{*}{ PEG2000 } & \multirow{2}{*}{$\begin{array}{c}3400 \\
13.6 \\
7.0\end{array}$} & \multirow{2}{*}{$\begin{array}{c}103 \\
0.68 \\
7.0\end{array}$} & $\begin{array}{l}177 \\
1.36\end{array}$ & $\begin{array}{l}10362 \\
5.16\end{array}$ & $\begin{array}{l}915 \\
7.74\end{array}$ & \multirow[t]{2}{*}{60} & \multirow[t]{2}{*}{300} \\
\hline & & & & & $\mathrm{V}_{\text {total }}=28.3$ & & & \\
\hline
\end{tabular}

The initial residence time was set to $210 \mathrm{~s}$ (total flow rate of $224.3 \mu \mathrm{L} / \mathrm{min}$ ) and the conversion was determined by in-line monitoring as described above. If the conversion did not reach completeness the residence time was stepwise increased by $30 \mathrm{~s}$ (individual flow rates can be derived from Table S8). The reactor output was directly precipitated in petroleum ether and dried under reduced pressure. The crude sample was dissolved in THF and precipitated twice in $\mathrm{MeOH}$ containing $4 \mathrm{vol} \%$ TFA. In all cases, white solids were obtained after filtration and drying the materials under reduced pressure. The products were characterized by ${ }^{1} \mathrm{H} \mathrm{NMR}$, ${ }^{13} \mathrm{C}$ NMR, FTIR, GPC, and DSC. 
Table S10: Experimental data for large-scale PU syntheses using continuous flow reactors and applying end-capping with HEMA for control over molecular weight.

\begin{tabular}{|c|c|c|c|c|c|c|}
\hline ID & $\begin{array}{c}\text { Conversion } \\
(\%)^{\mathrm{a}}\end{array}$ & $\begin{array}{l}x_{H D O} \\
(\%)^{\mathrm{a}}\end{array}$ & $\begin{array}{c}x_{H E M A} \\
(\%)^{\mathrm{a}}\end{array}$ & $\begin{array}{c}\text { Theoretical } M_{n} \\
(\mathrm{~kg} / \mathrm{mol})^{b}\end{array}$ & $\underset{(\mathrm{kg} / \mathrm{mol})^{\mathrm{c}}}{\operatorname{Experimental}} M_{n}$ & $\mathbf{D}^{\mathbf{c}}$ \\
\hline FlowPU8 & $>99$ & 60 & 4.0 & 24.5 & 18 & 1.4 \\
\hline FlowPU9 & $>99$ & 81 & 3.8 & 16.0 & 23 & 1.7 \\
\hline FlowPU10 & $>99$ & 63 & 5.5 & 28.8 & 33 & 1.5 \\
\hline FlowPU11 & $>99$ & 60 & 3.8 & 22.4 & 18 & 1.6 \\
\hline
\end{tabular}

\subsection{Thermal crosslinking of HEMA-endcapped PUs}

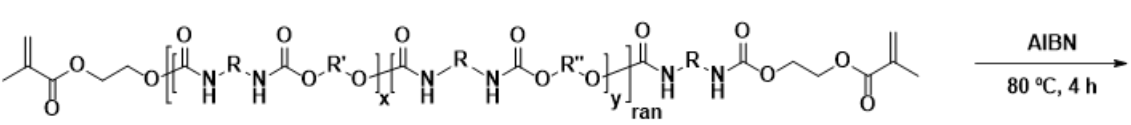

$$
\begin{aligned}
& \text { : 11 } \\
& \text { R: } \quad X_{6} \\
& R^{n}: \uparrow^{O} \overbrace{n-1} \text { PEG } \\
& \text { or }
\end{aligned}
$$
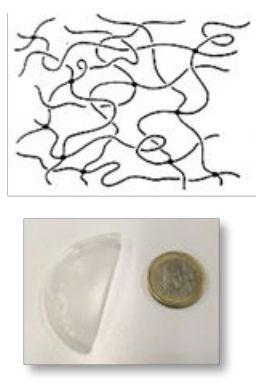

In a $25 \mathrm{~mL}$ glass vial, $2.00 \mathrm{~g}$ polymer (FlowPU8-11) and $30 \mathrm{mg}$ AIBN (1.5 weight-\%) were dissolved at room temperature in the minimum amount of THF and the solution transferred into PTFE dishes with a diameter of $5 \mathrm{~cm}$. After the solvent was removed under reduced pressure the sample was heated to $80{ }^{\circ} \mathrm{C}$ under nitrogen atmosphere and cured for $4 \mathrm{~h}$. The product was insoluble in THF and characterized by FTIR.
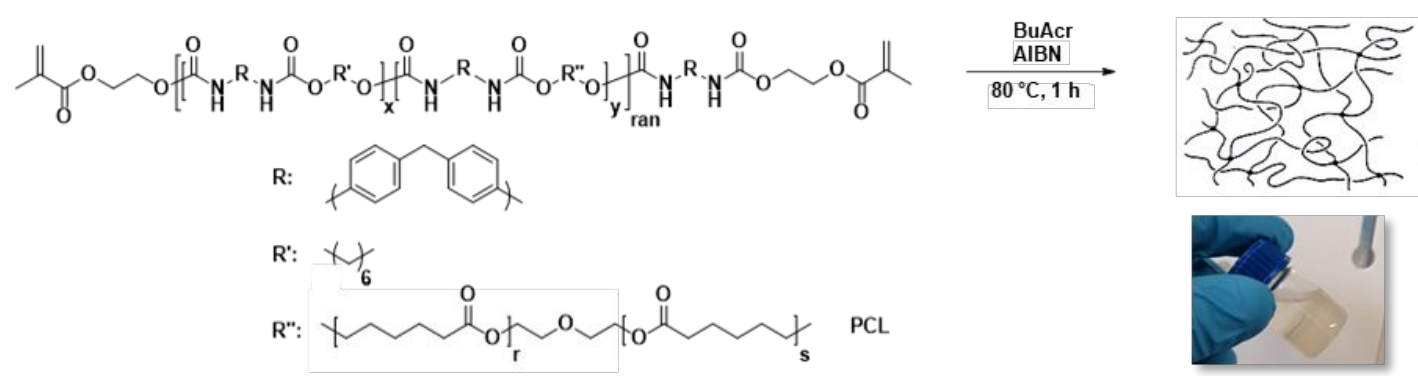
In a $5 \mathrm{~mL}$ glass vial, $713 \mathrm{mg}$ of FlowPU9 (71 wt\%) and $238 \mathrm{mg}$ of butyl acrylate (BuAcr, $24 \mathrm{wt} \%)$ were dissolved in the minimum amount of THF ( $2 \mathrm{~mL})$. After adding $50 \mathrm{mg}$ AIBN ( $5 \mathrm{wt} \%$ ) the mixture was heated to $80{ }^{\circ} \mathrm{C}$ for $1 \mathrm{~h}$ under nitrogen atmosphere. Afterwards, the reaction mixture was cooled to room temperature and the obtained gel was washed and dried in vacuum. The product was insoluble in THF.

\subsection{UV cross-linking of HEMA-endcapped PUs}
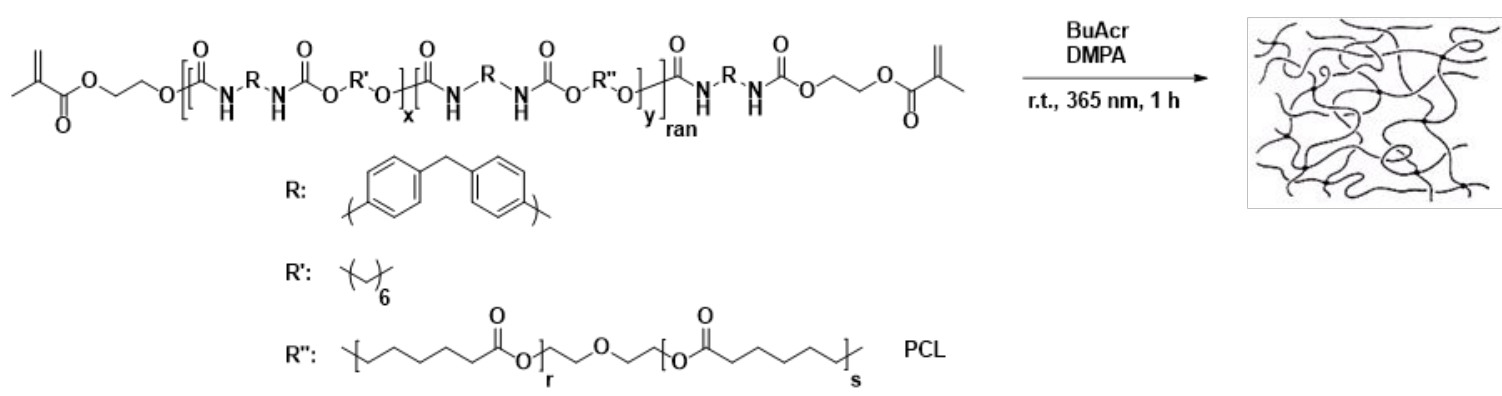

In a $5 \mathrm{~mL}$ glass vial, $713 \mathrm{mg}$ of FlowPU8-10 (71 wt $\%$ ), $238 \mathrm{mg}$ of butyl acrylate (BuAcr, $24 \mathrm{wt} \%)$ and $50 \mathrm{mg}(5 \mathrm{wt} \%)$ of DMPA were dissolved in the minimum amount of THF ( 2 $\mathrm{mL})$. The solvent was evaporated until a viscous liquid was obtained which then was poured into silicon dishes with a diameter of $1 \mathrm{~cm}$ and irradiated for $1 \mathrm{~h}$ with a UV LED light centered at $365 \mathrm{~nm}$ (Hamamatsu, $100 \mathrm{~mW} \cdot \mathrm{cm}^{-2}$ ). Afterwards the crosslinked samples were dried under vacuum overnight.
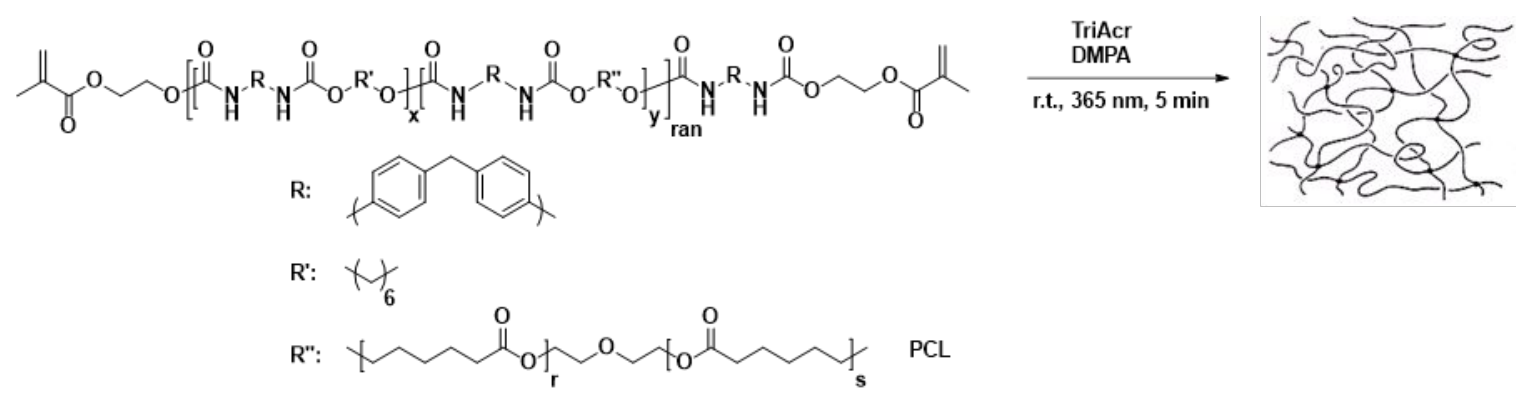

In a $5 \mathrm{~mL}$ glass vial, $713 \mathrm{mg}$ of FlowPU8-10 (71 wt\%), $238 \mathrm{mg}$ of trimethylolpropane triacrylate (TriAcr, $24 \mathrm{wt} \%)$ and $50 \mathrm{mg}(5 \mathrm{wt} \%)$ of DMPA were dissolved in the minimum 
amount of THF ( $2 \mathrm{~mL})$. The solvent was evaporated until a viscous liquid was obtained which then was poured into silicon dishes with a diameter of $1 \mathrm{~cm}$ and irradiated for $5 \mathrm{~min}$ with a UV LED light centered at $365 \mathrm{~nm}$ (Hamamatsu, $100 \mathrm{~mW} \cdot \mathrm{cm}^{-2}$ ). Afterwards the crosslinked samples were dried under vacuum overnight.

Table S11: Gel content of the UV crosslinked materials after Soxhlet extraction for $24 \mathrm{~h}$.

\begin{tabular}{cc}
\hline Entry & Gel content (\%) \\
\hline FlowPU8-BuAc & $71 \pm 1$ \\
FlowPU9-BuAc & $72 \pm 3$ \\
FlowPU10-BuAc & $72 \pm 1$ \\
FlowPU8-TriAc & $84 \pm 1$ \\
FlowPU9-TriAc & $82 \pm 1$ \\
FlowPU10-TriAc & $85 \pm 1$ \\
\hline
\end{tabular}

\subsection{Batch polymerization: Large-scale PU synthesis with end-capping by HEMA}

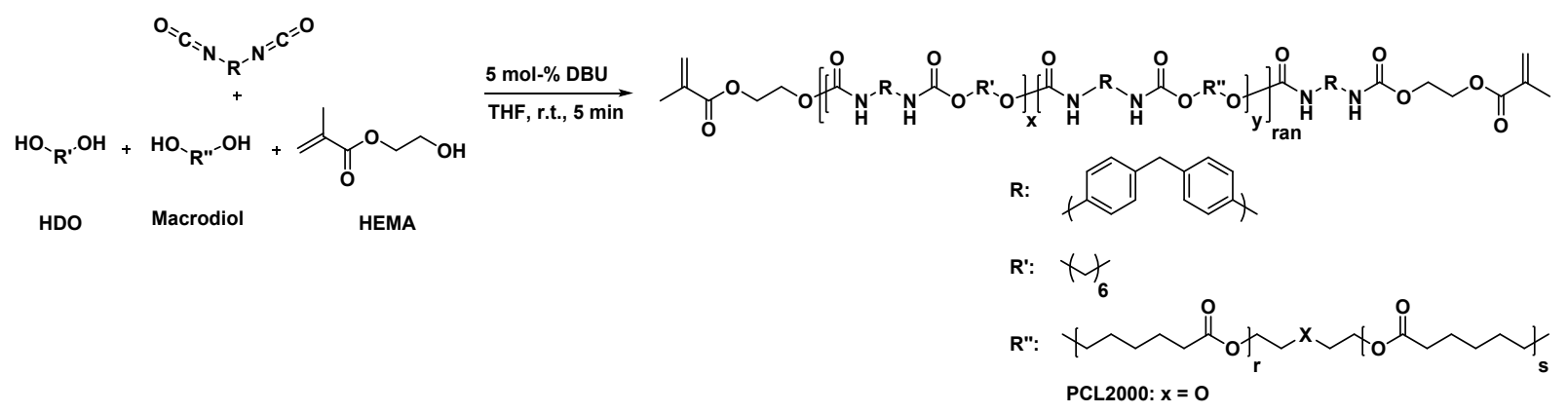

In a $\mathrm{N}_{2}$-filled glovebox a solution of PCL2000, HDO and HEMA (molar ratio for $x_{H D O, t h}=$ $60 \%$ : 3.8:5.7:1.0, total concentration $0.48 \mathrm{M}$ ) with HEMA taking up 5 mol-\% of the total alcohol groups was prepared by dissolving $2.54 \mathrm{~g}$ (21.52 mmol, 0.57 eq.) HDO, $32.60 \mathrm{~g}$ (14.35 mmol, 0.38 eq.) PCL2000, and $0.49 \mathrm{~g}$ (3.78 mmol, 0.10 eq.) HEMA in THF to a total volume of $82.6 \mathrm{~mL}$. A 1.92 M solution of MDI was prepared by dissolving $9.45 \mathrm{~g}(37.75 \mathrm{mmol}$, 
1.00 eq.) MDI in THF to a total volume of $19.7 \mathrm{~mL}$ and a $0.096 \mathrm{M}$ solution of $\mathrm{DBU}$ by dissolving $0.29 \mathrm{mg}$ (1.89 mmol, 0.05 eq.) DBU in THF to a total volume of $19.7 \mathrm{~mL}$.

To run three individual batch polymerizations at a scale of about $15 \mathrm{~g}$, the solutions were divided into three equal parts and injected into a 100mL-round bottom flask at room temperature in the order: PCL - DBU - MDI. Under vigorous stirring each reaction was ran for 5 min while an increase in temperature and viscosity was observed. Afterwards, a small sample of the reaction mixture as well as the reaction mixture were added into diethyl ether to quench the reaction by precipitating the reaction components. The solvents in the former were removed by a stream of air and under reduced pressure at room temperature and the crude material was analyzed by ${ }^{1} \mathrm{H}$ NMR for determining the conversion and by APC for molecular weights and molecular weight distribution.

The main part of the crude reaction material was purified by three precipitations from THF into diethyl ether and dried under reduced pressure at room temperature resulting in a white solid (yield: 13.1-14.6 g, 87-97\%).

Table S12: Results of large-scale batch polymerizations using HDO/PCL2000 (60:40).

\begin{tabular}{|c|c|c|c|c|c|}
\hline & BatchPU14 & BatchPU15 & BatchPU16 & Mean & Std Dev \\
\hline Conversion p $(\%)^{a}$ & 99.9 & 94.9 & 87.8 & 94.1 & 5.9 \\
\hline$x_{H D O, t h}(\%)^{\mathrm{b}}$ & 60.0 & 60.0 & 60.0 & & \\
\hline$x_{H D O, \exp }(\%)^{\mathbf{b}}$ & 60.6 & 57.5 & 56.1 & & \\
\hline$\Delta x_{H D O}(\%)$ & 0.6 & -2.5 & -3.9 & -2.0 & 2.3 \\
\hline$x_{H E M A, t h}(\%)^{\mathrm{c}}$ & 9.52 & 9.52 & 9.52 & & \\
\hline$x_{H E M A, \exp }(\%)^{\mathrm{c}}$ & 6.6 & 6.2 & 6.8 & & \\
\hline$\Delta x_{H E M A}(\%)$ & -2.9 & -3.3 & -2.7 & -3.0 & 0.3 \\
\hline$w_{H S, t h}(\%)^{\mathrm{d}}$ & 26.1 & 26.1 & 26.1 & & \\
\hline$w_{H S, \exp }(\%)^{\mathrm{d}}$ & 26.4 & 24.8 & 24.1 & & \\
\hline$\Delta w_{H S}(\%)$ & 0.3 & -1.3 & -2.0 & -1.0 & 1.2 \\
\hline
\end{tabular}




\begin{tabular}{|c|c|c|c|c|c|}
\hline$M_{r u, t h}(\mathrm{~g} / \mathrm{mol})^{\mathrm{e}}$ & 1229.95 & 1229.95 & 1229.95 & & \\
\hline$M_{r u, \exp }(\mathrm{g} / \mathrm{mol})^{\mathrm{f}}$ & 1217.25 & 1284.32 & 1314.67 & & \\
\hline$M_{n, t h}(\mathbf{k D a})^{\mathrm{e}}$ & 24.25 & 8.30 & 4.29 & & \\
\hline$M_{n, \exp }(\mathrm{kDa})^{\mathrm{f}}$ & 34.28 & 9.72 & 4.79 & & \\
\hline$\Delta M_{n}(\%)$ & 41.4 & 17.2 & 11.7 & 23.4 & 15.8 \\
\hline$M_{n, G P C}(\mathrm{kDa})^{\mathrm{g}}$ & 32.54 & 19.9 & 13.79 & & \\
\hline $\boldsymbol{\oplus}_{G P C^{g}}$ & 1.4 & 1.62 & 1.71 & & \\
\hline
\end{tabular}

$\bar{a}$ From crude sample applying Equation S4. ${ }^{\mathrm{b}}$ See Equation S6. ${ }^{\mathrm{c}}$ See Equation S10. ${ }^{\mathrm{d}}$ See Equation S11. ${ }^{\mathrm{e}}$ See Equation S19 using $x_{H E M A, t h}=0.0952, p$ as listed, and $M_{r u, t h}$ calculated with $x_{H D O, t h .} .{ }^{\mathrm{f}}$ See Equation S19 using experimental $x_{H E M A, \exp }$ and $M_{r u, \exp }$ calculated with $x_{H D O, \text { exp }} . \mathrm{g}$ Determined by APC vs PS standard.

Table S11 shows the results of large-scale batch polymerizations analogous to the continuousflow synthesis of FlowPU8. Monomer conversion and material composition were derived from processing ${ }^{1} \mathrm{H}$ NMR spectra and molecular weights and molecular weight distributions were obtained from APC measurements. Compared with flow polymerizations higher deviations from theoretical values were observed. For example, in comparison to the conducted flow polymerizations (Table 2) $\Delta x_{H D O}$ is five times larger $(-2.0 \pm 2.3 \% \mathrm{vs}-0.4 \pm 2.2 \%), \Delta x_{H E M A}$ is about three times larger $(-3.0 \pm 0.3 \%$ vs. $-0.9 \pm 1.4 \%)$ and the conversion ranges from $99.9 \%$ to $87.8 \%$ with an average around $94.1 \pm 5.9 \%$. Since these three values mainly determine molecular weights and physical material properties for the studied segmented PUs it is most important to have good control over the same and to reach theoretical values. Conducting the syntheses in flow therefore is advantageous not only due to higher reproducibility and realtime monitoring but also due to the ability to fractionate the product during the experiment. Furthermore, using continuous-flow here also increases the efficiency of monomer/material usage by reducing the amount of PU material not fulfilling theoretically determined properties. 


\section{Investigation of process control employing PID controllers}

As previously described, we placed an ATR-FTIR sensor downstream the entrance point of the reactor in which the monomers and DBU were mixed. The reactor length was $105 \mathrm{~cm}$ with a typical flow rate of $0.165 \mathrm{~mL} / \mathrm{min}$. Assuming the plug flow approximation, this yields a residence time of $5 \mathrm{~min}$. Given the vibrational characteristics of MDI with the asymmetric NCO stretching centered at about $2270 \mathrm{~cm}^{-1}$, this allowed us to directly calculate the conversion of the reaction (section 7.2.3) and to investigate employing this signal to control the process at a particular conversion. Higher conversion is to be expected for diminished flow rate and low conversions for high flow rates.

An obvious difficult of this system is the long and variable dead time of about $5 \mathrm{~min}$ between process changes (flow rates) and FTIR measurement at the end of the reactor. Thus, a PID controller taking its signal directly from the sensor would afford a poor control of the process. The Smith predictor algorithm is the typical solution to such a problem. In this scheme, a process model is used to compute the process output in the future and this prediction, if perfect, can be used as the input to the PID controller. By employing this model prediction, the dead time is removed and the PID algorithm receives immediate feedback to process changes. In practice the model is never perfect, so if only the model prediction is used there will be an offset between the model and the eventual steady state process value. Because of this mismatch, the Smith predictor uses the model's future predicted output plus the model's current prediction as compared to the measured value at the sensor. Thus, the controller acts on a signal that mitigates the dead time problem and any discrepancy between the model and actual measurement (Figure S19). 


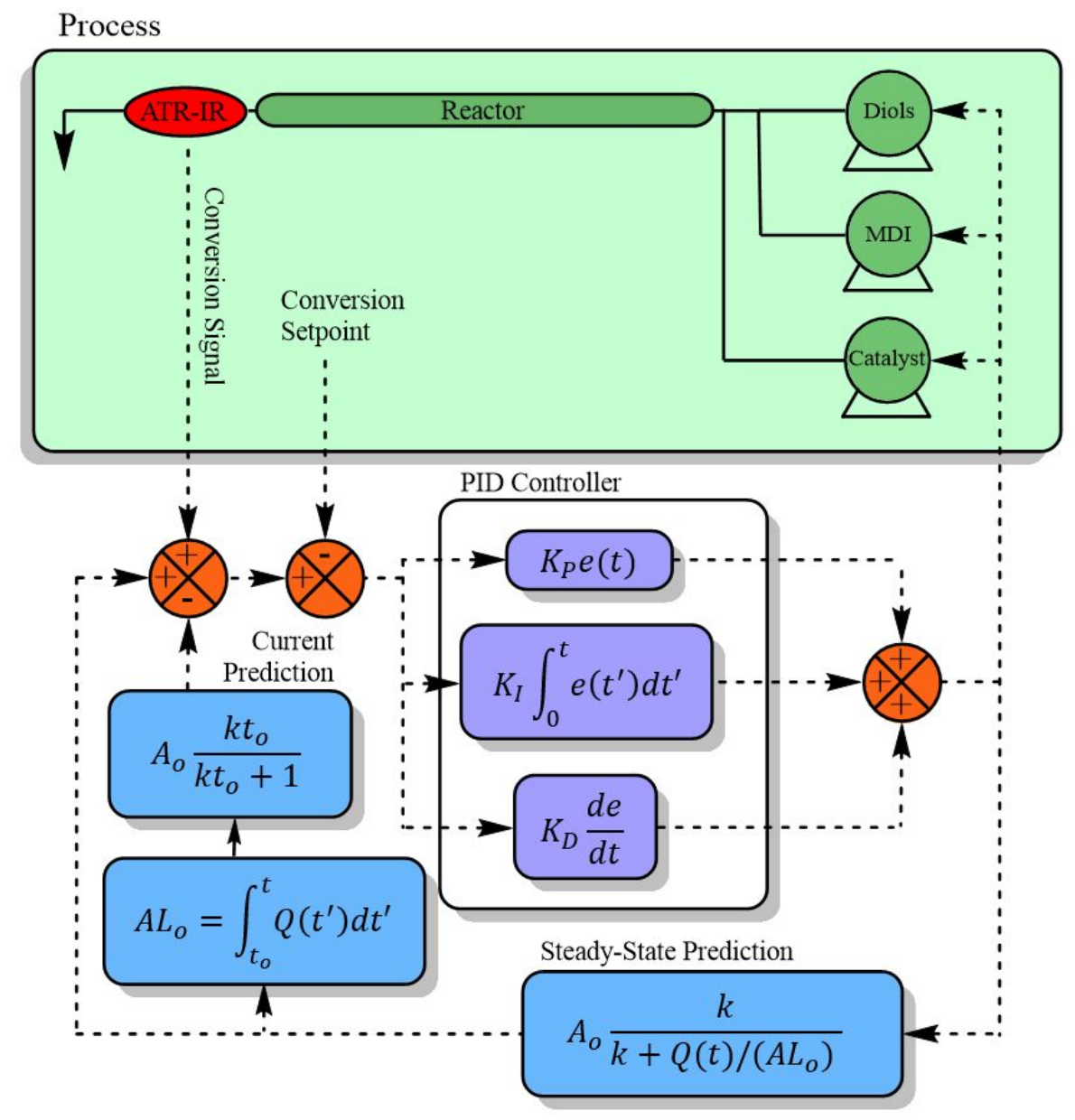

Figure S19: The PID controller sets the total flow rate (Q) for the three pumps. The ATRFTIR is located at the end of the reactor having length $\mathrm{L}_{0}$ and cross-sectional area $\mathrm{A}$. The Smith predictor elements (blue) modify the input signal of the PID controller to negate the effect of the dead time on controller performance.

We attempted this control scheme. The process model employed was a simple $2^{\text {nd }}$ order kinetic scheme employing a rate constant value giving $90 \%$ conversion at 5 minutes for an initial MDI concentration of $0.32 \mathrm{M}$. The plug flow approximation was employed to compute the reaction/residence time at the FTIR sensor. The expected inverse relationship between conversion at the sensor and the flow rate is described by Equation S23 


$$
c=\frac{A_{o}}{1+\frac{Q(t)}{A k L_{o} A_{o}}}
$$

where $\mathrm{Q}(\mathrm{t})$ is the flow rate at the pump, $\mathrm{A}$ is area of the reactor, $\mathrm{k}$ is the kinetic constant, $\mathrm{L}_{\mathrm{o}}$ is the reactor length, and $A_{o}(1.0)$ is the stoichiometric equivalents relative to a reference concentration $(0.32 \mathrm{M})$ and returns the expected steady state or future conversion for the given flow rate.

Likewise, numerically solving Equation S24 for time allows us to compute the current expected conversion at the sensor (Equation S25) so that we can compute the discrepancy between the model and the current measurement.

$$
\begin{gathered}
\int_{0}^{t} Q\left(t^{\prime}\right) d t^{\prime}=L_{o} A \\
c=\frac{A_{o}}{1+\frac{1}{k t A_{o}}}
\end{gathered}
$$

With these two relationships, and a kinetic constant estimated to be $1.8 \mathrm{~min}^{-1}$, we had sufficient information to build a controller incorporating a smith predictor element. However, when we tried to control the process at a given setpoint we found that the controller itself gave improper control. In particular, if the process was at near steady state at high conversion $(>90 \%)$ and the flow rate was suddenly increased we noted an immediate increase in conversion (lower MDI concentration measured at the FTIR sensor) before decreasing to the expected reduced conversion (higher MDI concentration measured at the FTIR) (Figure S20). This inverse response behavior was not anticipated in our process model. 


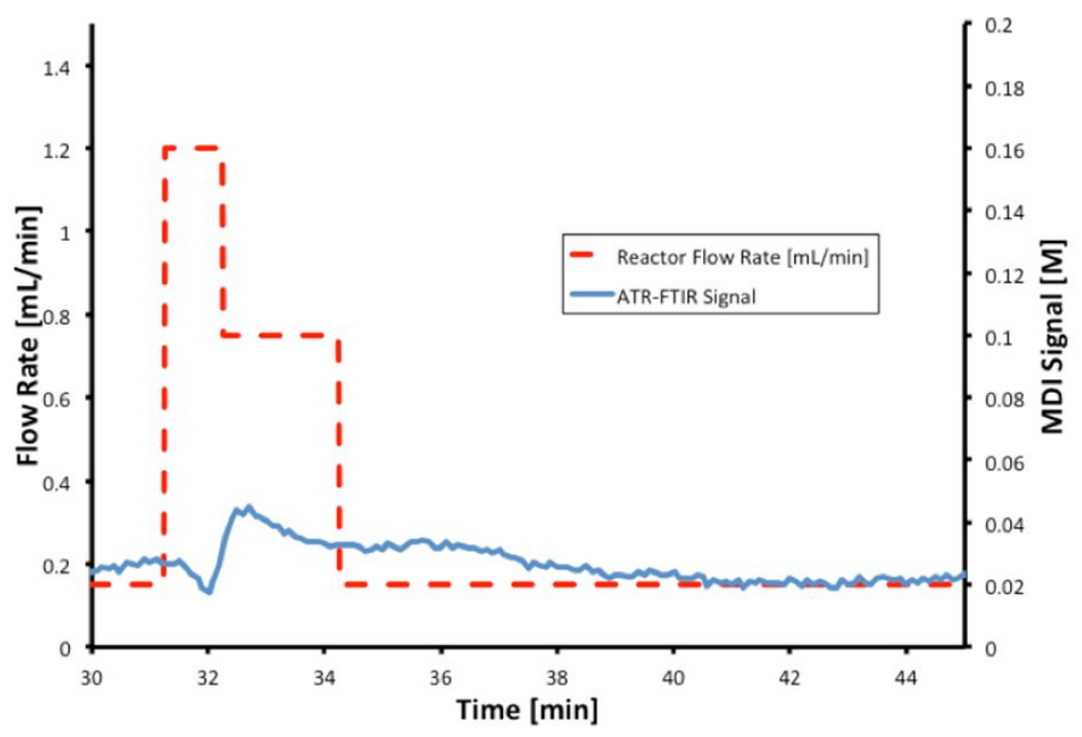

Figure S20: Diagram showing the observed inverse response behavior for the DBU-catalyzed (5 mol-\%) preparation of PUs in flow from MDI and HDO/PCL (molar ratio 80:20). At minute 31 at which the measured signal had achieved an apparent near steady-state, the total flow rate was increased from $0.175 \mathrm{~mL} / \mathrm{min}$ to $1.2 \mathrm{~mL} / \mathrm{min}$. The measured FTIR signal and derived MDI concentration saw a decrease followed by an increase. As higher flow rates should lead to lower monomer conversions and, thus, higher MDI concentrations at the FTIR sensor, the obtained measurement signal is inverse to the expectations and does not follow the pattern for plug flow behavior.

We initially hypothesized that the fluid flow exhibited some kind of channeling flow at the sensor that would give an erroneous signal. Therefore, we attempted an experiment wherein we replaced our reactive solution with a solution of polyethylene oxide in chloroform and a second syringe with pure acetone. The flow rate of both solutions was systematically varied and the characteristic resonance signal of acetone at $1710 \mathrm{~cm}^{-1}$ was recorded. A control experiment where polymer was not included was also performed. As shown in the Figure S21, the control experiment without polymer exhibited composition changes consistent with the plug flow approximation. The solution with polymer showed a very similar behavior but "softer" 
transitions are indications for the presence of back-mixing. At high flow rates, the polymer solution exhibited erratic signals for which we do not have a clear mechanistic explanation.

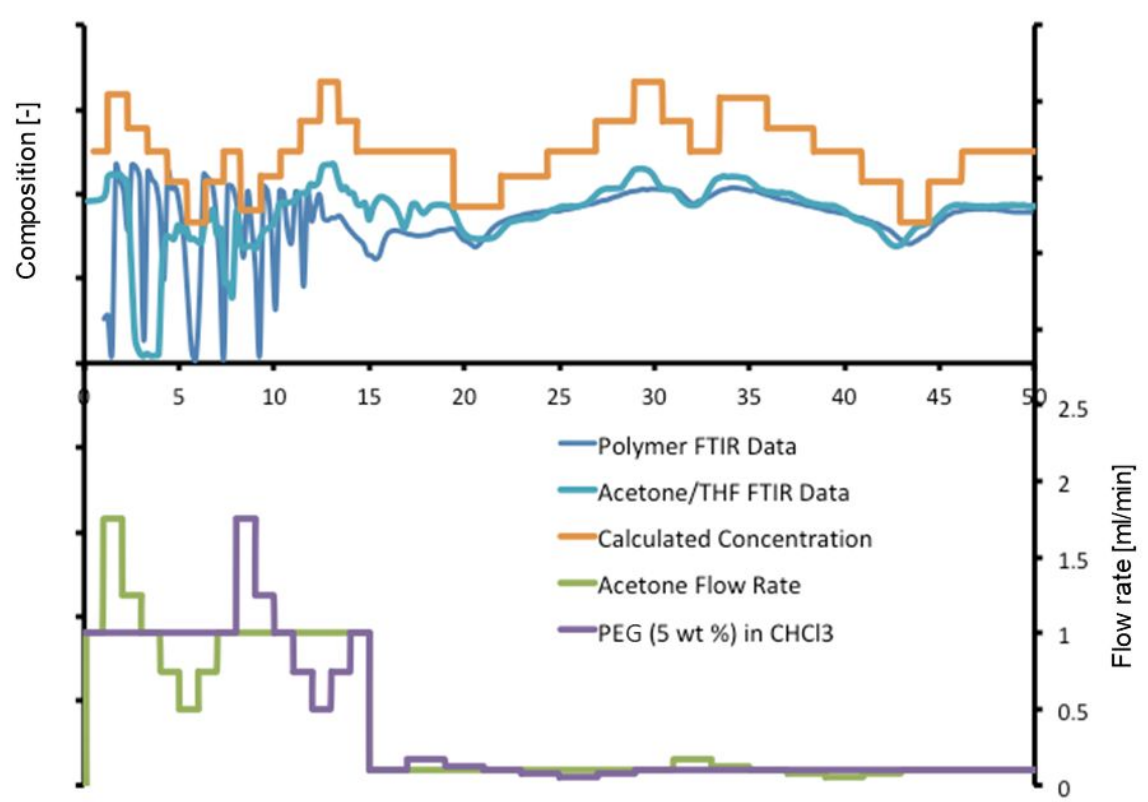

Figure S21: Results of control experiments performed using unreactive compounds (acetone and PEG in $\mathrm{CHCl}_{3}$ ) and mixtures with THF thereof. The experimental setup consisted of two syringe pumps to alter the mixing ratio of the solutions/fluids (acetone or PEG/CHCl3 to THF) injected into a common tube with a length of $100 \mathrm{~cm}$ and $1 \mathrm{~mm}$ inner diameter. The diagram presents changes in composition (top) in comparison to changes in flow rates (bottom). The orange line indicates the expected concentration of acetone based on plug flow approximation. The teal line is the acetone signal (dimensionless) as measured by the FTIR for an experiment in which the solutions were THF and acetone. The blue line is the acetone signal for an experiment in which the solutions were $5 \mathrm{wt}-\%$ PEG in $\mathrm{CHCl}_{3}$ and acetone.

Having done these experiments we believe instead that the observed inverse response behavior for control experiments employing the PU monomers is a behavior characteristic of a reactive solutions. Several computational studies have been performed ${ }^{12,13}$ describing the deviation 
from parabolic flow (seen for Newtonian liquids) for polymerizing solutions. Because fluid elements near the wall are characterized by longer residence times these fluid elements are characterized by a higher conversion and viscosity. Thus, the flow field is no longer parabolic and the flow in the middle of the tube is greater than what would be predicted for a parabolic flow field. Likewise, the flow near the wall is less than what would be predicted for a parabolic flow field. This change in flow field transitions from a parabolic flow at the beginning of the reactor towards the distorted flow field further down the reactor. Hence, at low flow rates and with increasing distortion of the flow field a significant amount of high conversion fluid is present near the reactor walls towards the end of the reactor. When the flow is now suddenly increased much of this high conversion fluid is "flushed" out of the reactor and the inline sensor system indeed immediately returns an "increase" in conversion - the unexpected inverse response behavior. Only afterwards, the decrease in conversion of the "fresh" fluid is observed due to the increased flow rate. There are some examples of inverse response behavior in the process control literature. Several papers have examined process control schemes that incorporate both inverse response and dead times. ${ }^{14-16}$ However, the development of such a controller would require significant modeling effort and is outside the scope of this manuscript. We are current investigating this behavior for a future publication. 
9. Experimental data: NMR, FTIR, DSC, GPC and Rheology

d)

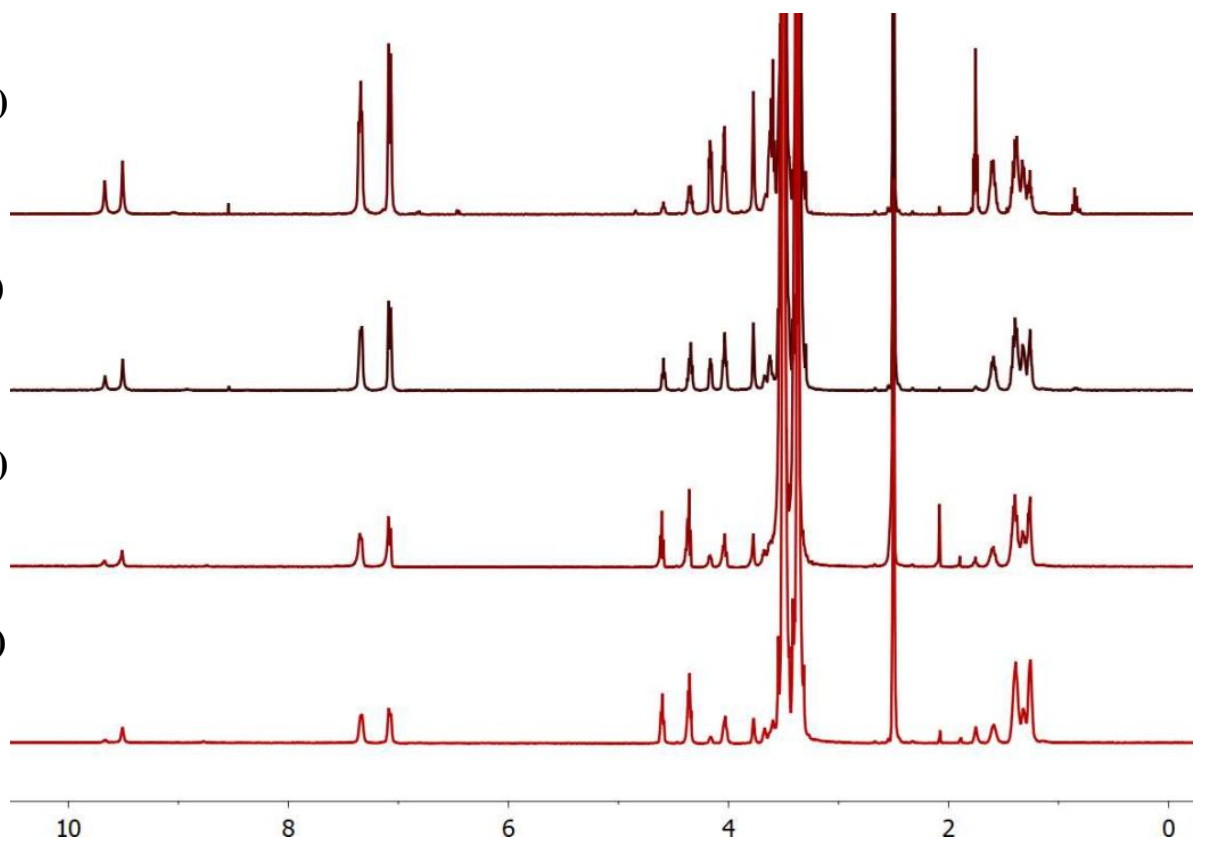

Figure S22: ${ }^{1} \mathrm{H}$ NMR (DMSO- $d_{6}$ ) of BatchPU1 at: a) 1 min, b) 5 min, c) 10 min and d) $20 \mathrm{~min}$.

e)

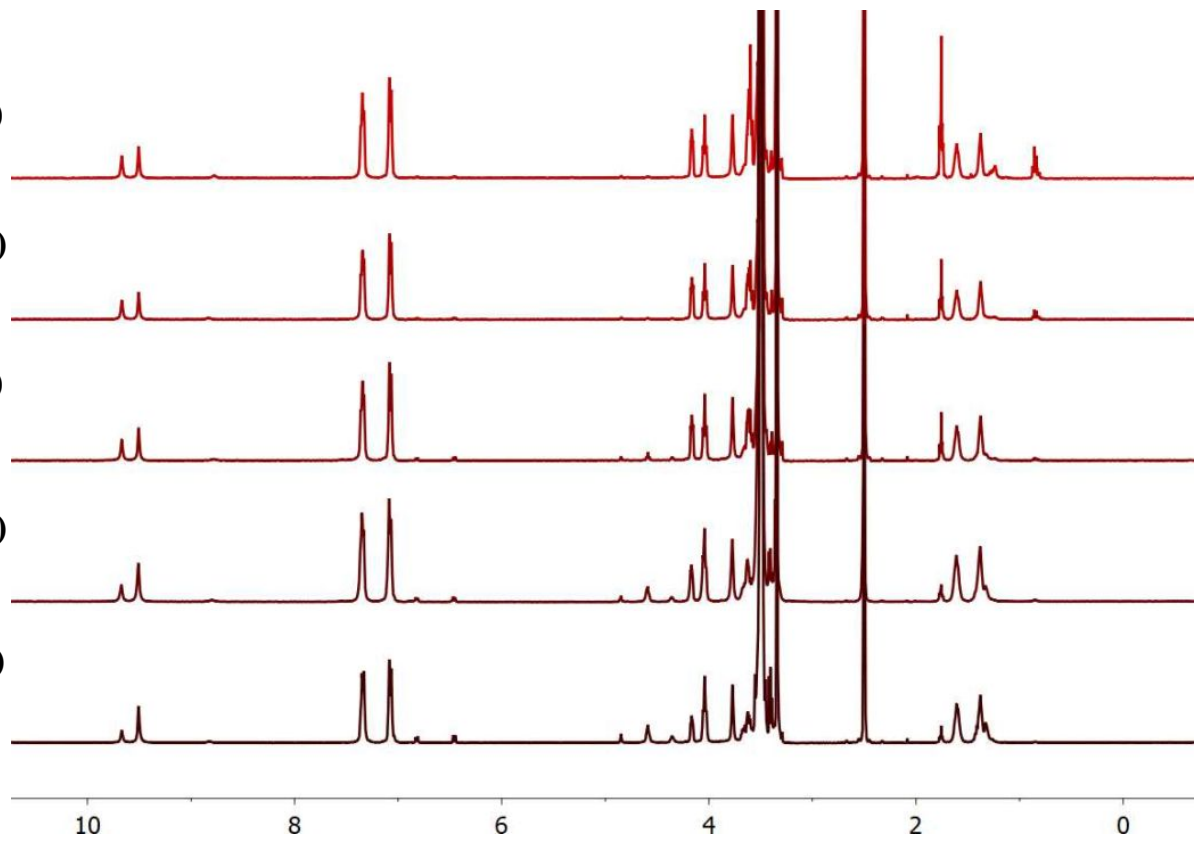

Figure S23: ${ }^{1} \mathrm{H}$ NMR (DMSO- $d_{6}$ ) of BatchPU2 at: a) 1 min, b) 2 min, c) 5 min, d) 10 min and e) $20 \mathrm{~min}$. 


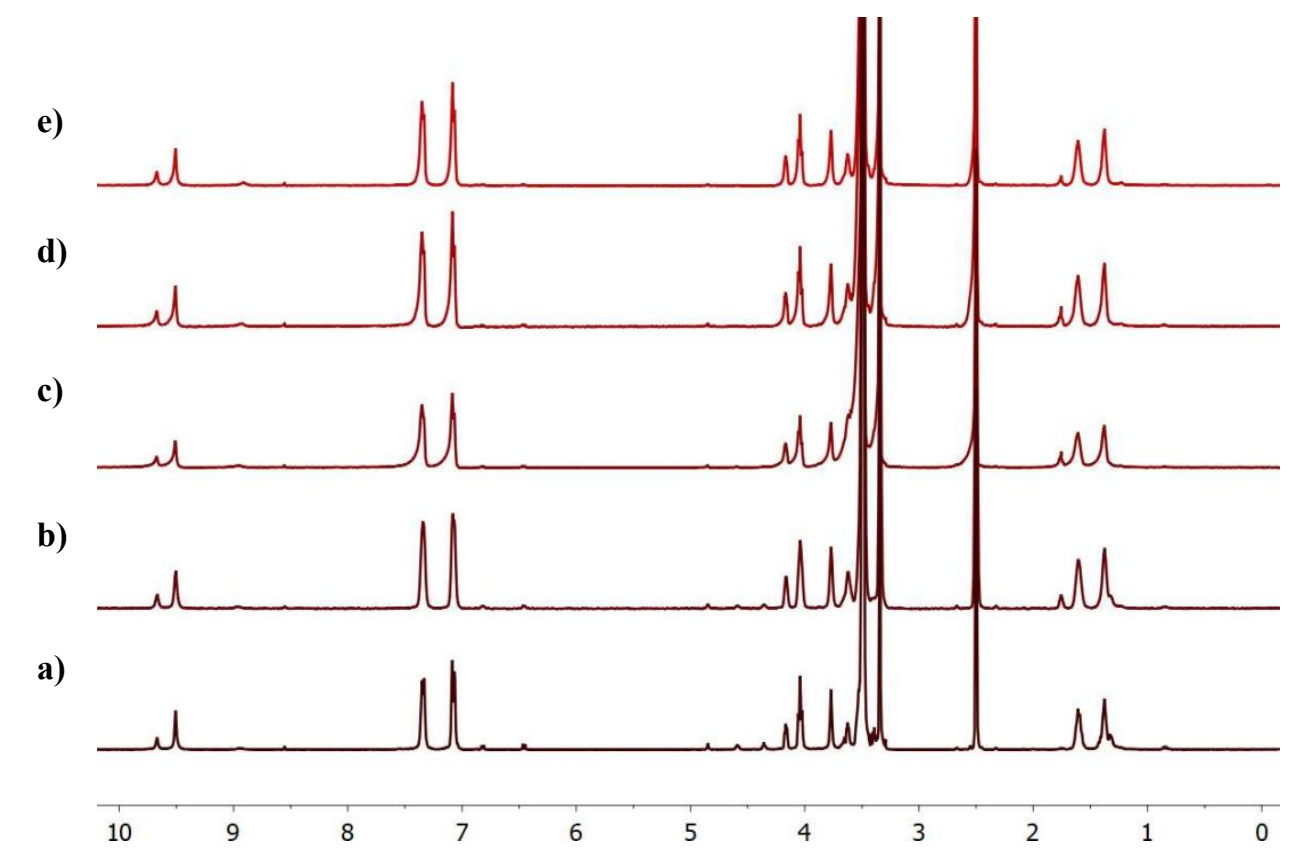

Figure S24: ${ }^{1} \mathrm{H}$ NMR (DMSO- $d_{6}$ ) of BatchPU3 at: a) 1 min, b) 2 min, c) 5 min, d) 10 min and e) $20 \mathrm{~min}$.

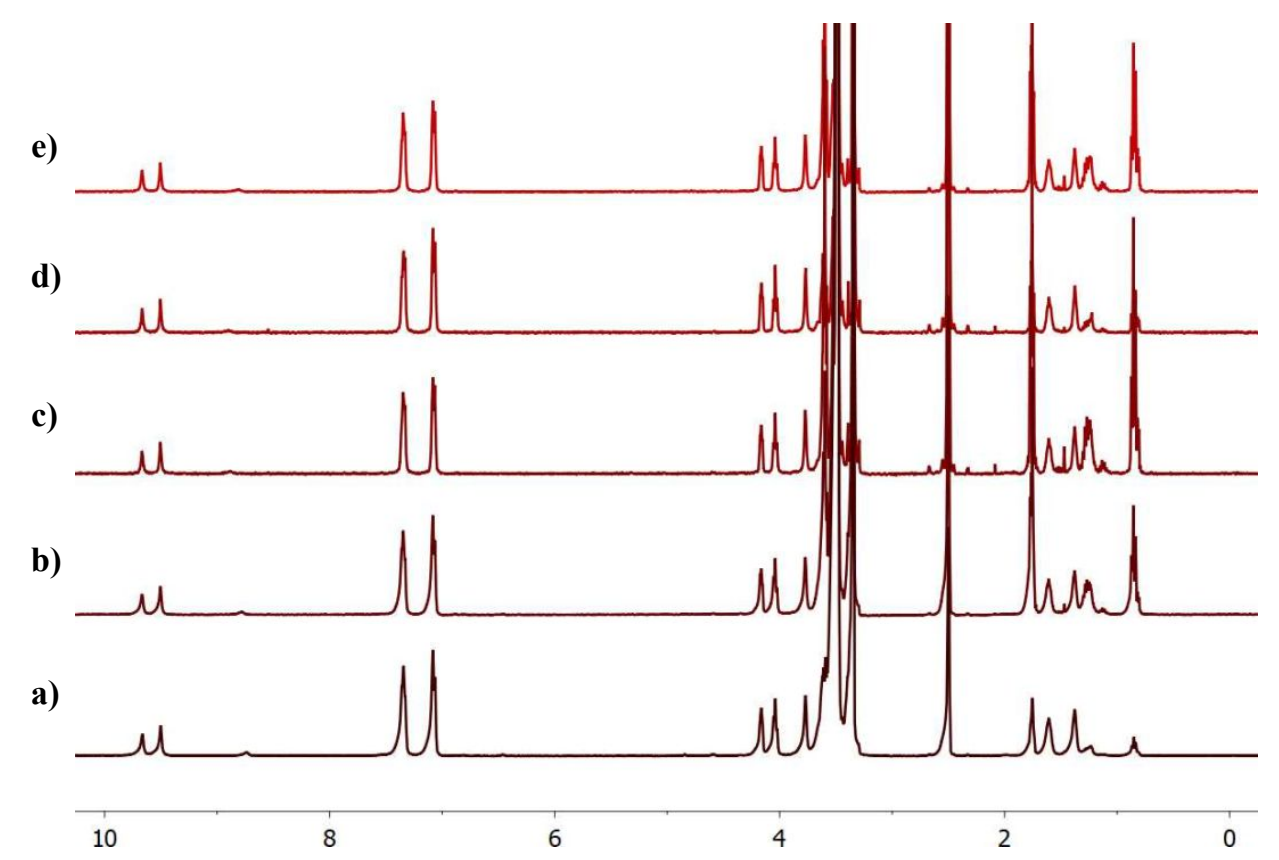

Figure S25: ${ }^{1} \mathrm{H}$ NMR (DMSO- $d_{6}$ ) of BatchPU4 at: a) 1 min, b) $2 \mathrm{~min}$, c) 5 min, d) $10 \mathrm{~min}$ and e) $20 \mathrm{~min}$. 


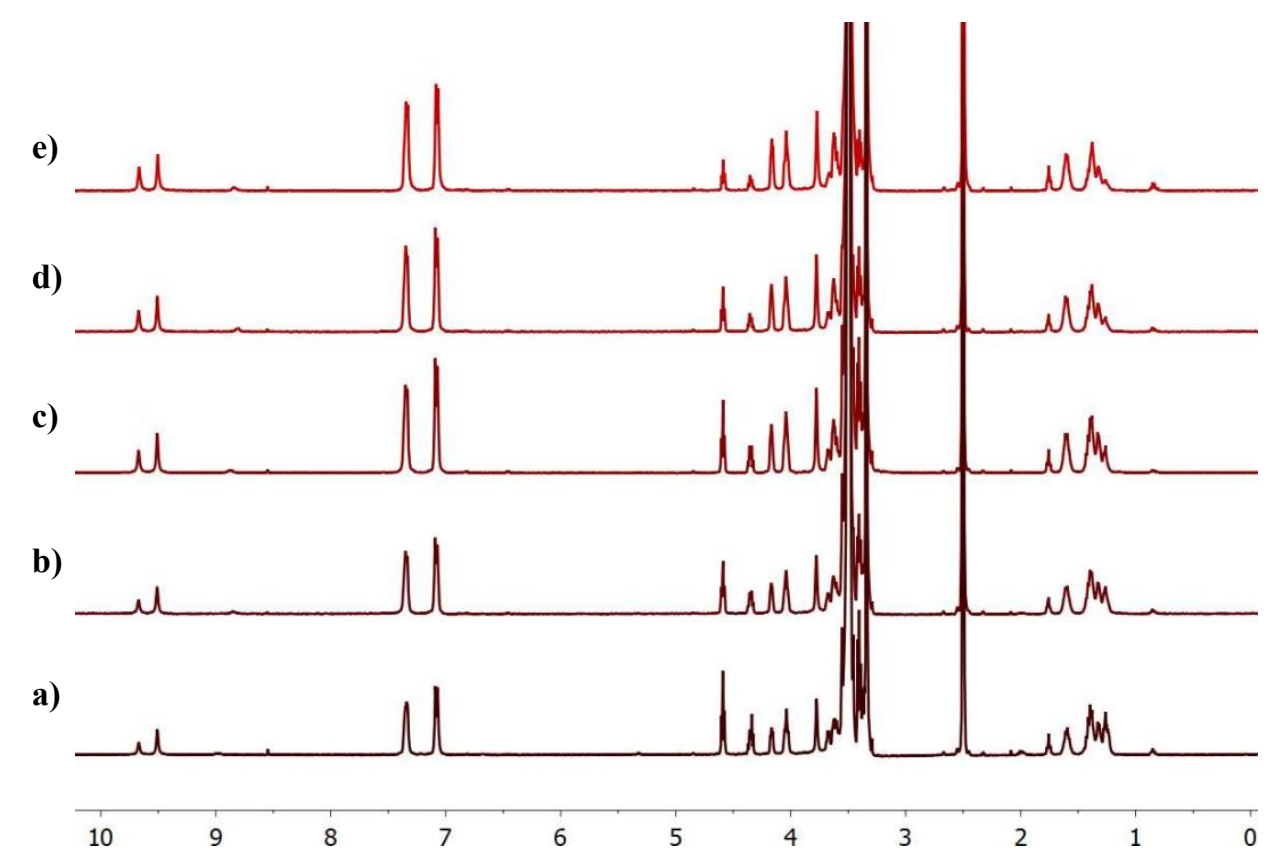

Figure S26: ${ }^{1} \mathrm{H}$ NMR (DMSO- $d_{6}$ ) of BatchPU5 at: a) 1 min, b) 2 min, c) 5 min, d) 10 min and e) $20 \mathrm{~min}$.

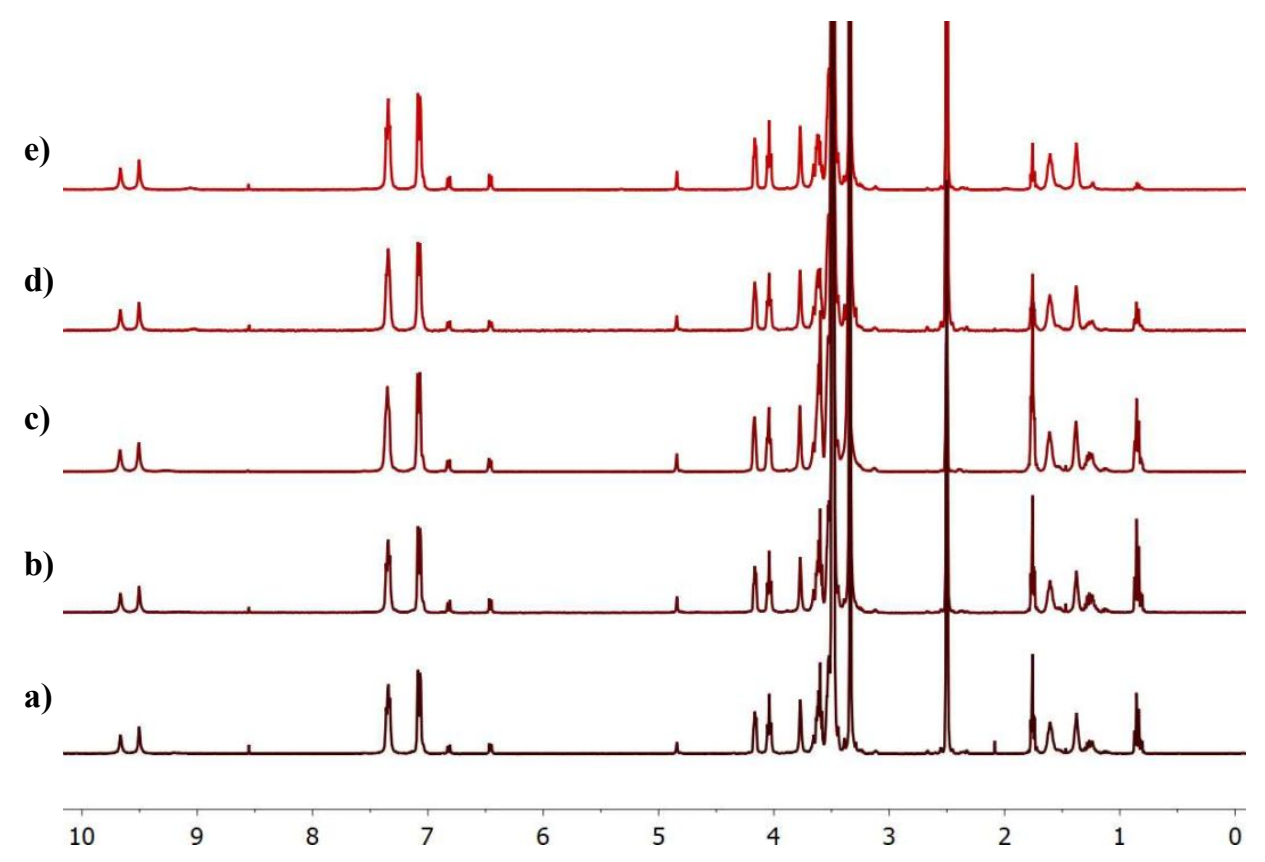

Figure S27: ${ }^{1} \mathrm{H}$ NMR (DMSO- $d_{6}$ ) of BatchPU6 at: a) 1 min, b) $2 \mathrm{~min}$, c) 5 min, d) $10 \mathrm{~min}$ and e) $20 \mathrm{~min}$. 


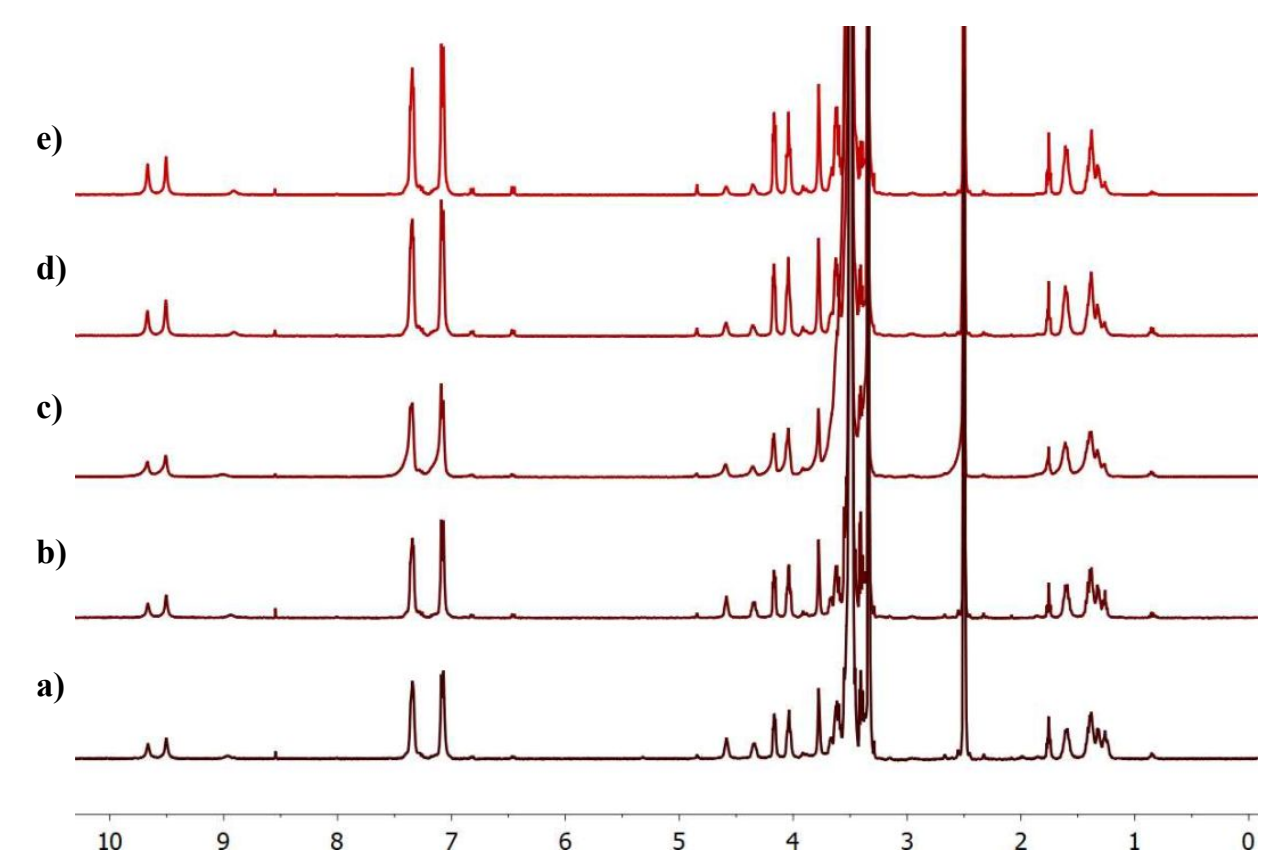

Figure S28: ${ }^{1} \mathrm{H}$ NMR (DMSO- $d_{6}$ ) of BatchPU7 at: a) 1 min, b) 2 min, c) 5 min, d) 10 min and e) $20 \mathrm{~min}$.

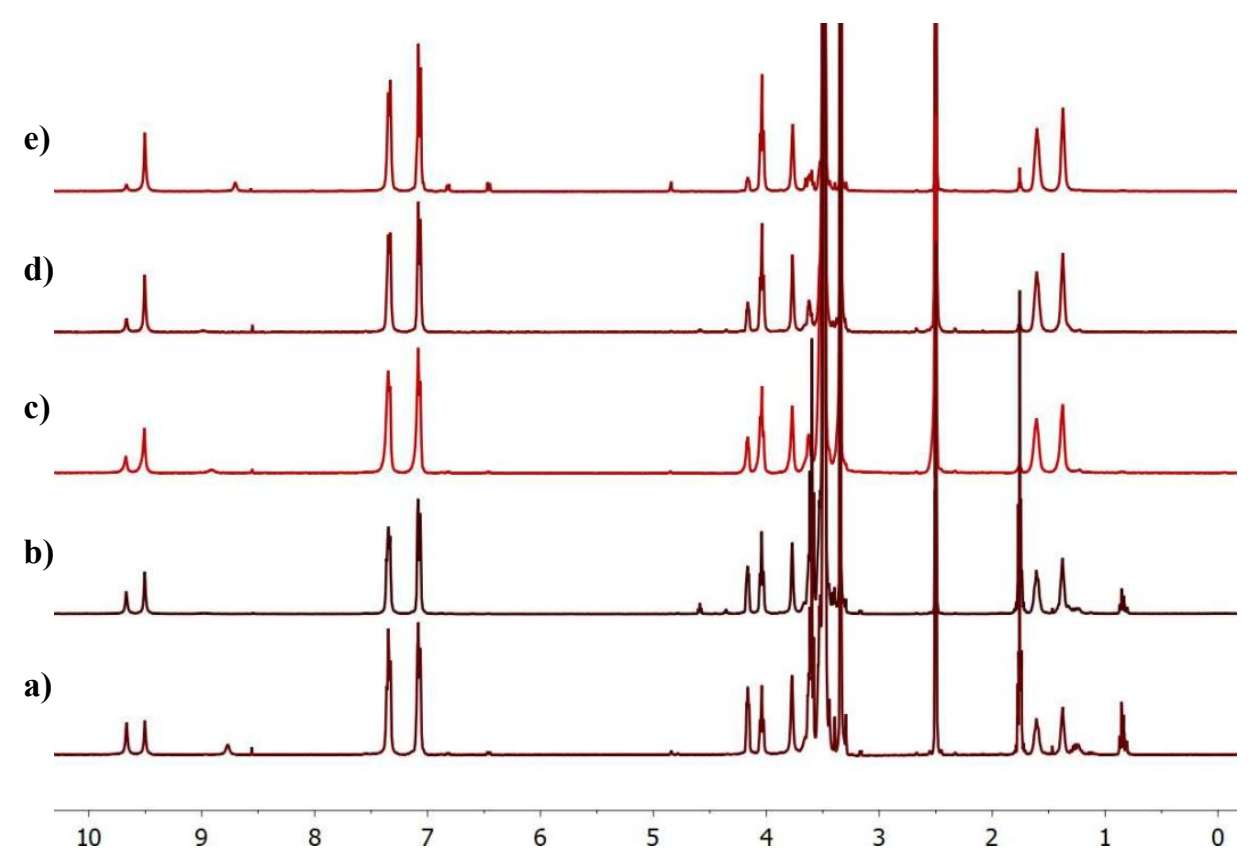

Figure S29: ${ }^{1} \mathrm{H}$ NMR (DMSO- $d_{6}$ ) of PUs varying HS \%: a) BatchPU8, b) BatchPU9, c) BatchPU10, d) BatchPU11 and e) BatchPU12. 


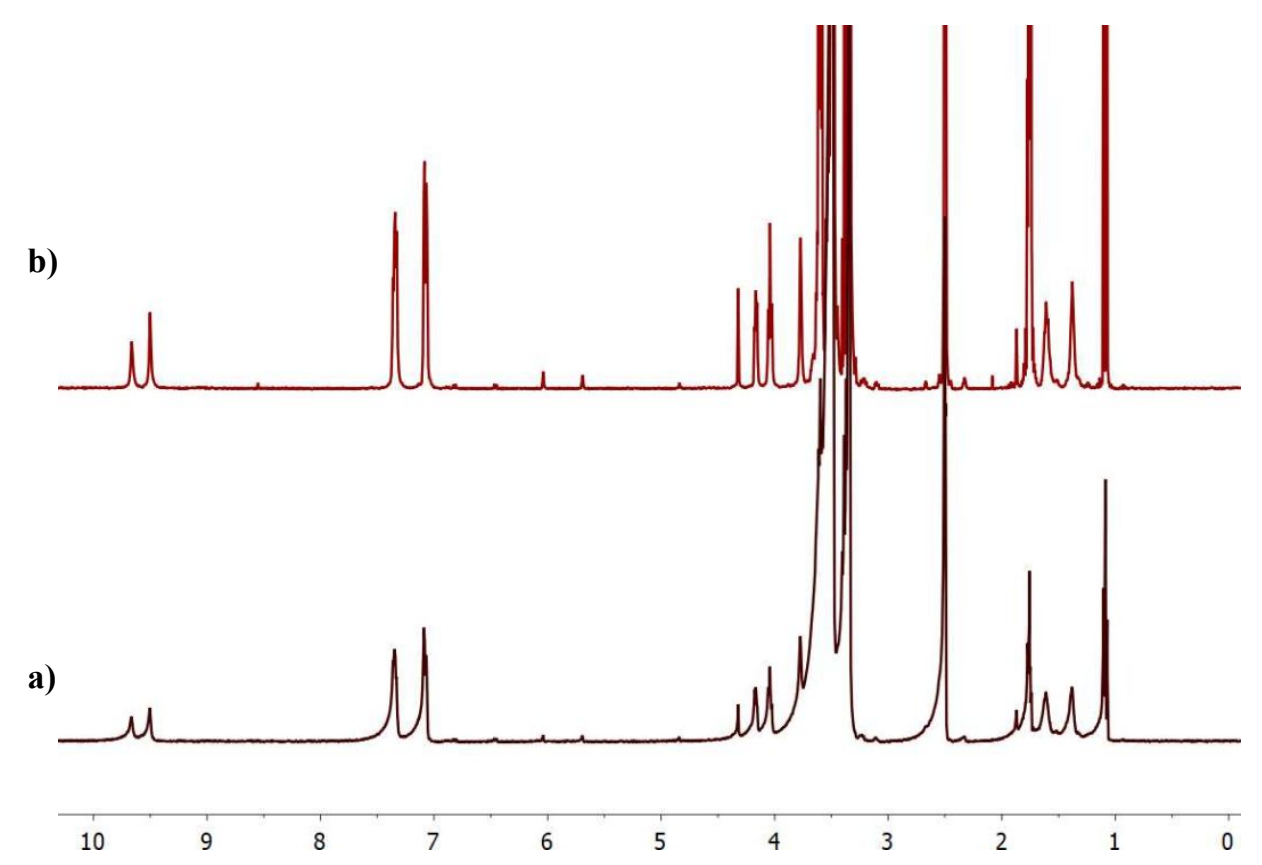

Figure S30: ${ }^{1} \mathrm{H}$ NMR (DMSO- $d_{6}$ ) of BatchPU13 at: a) 1 min, b) 10 min.

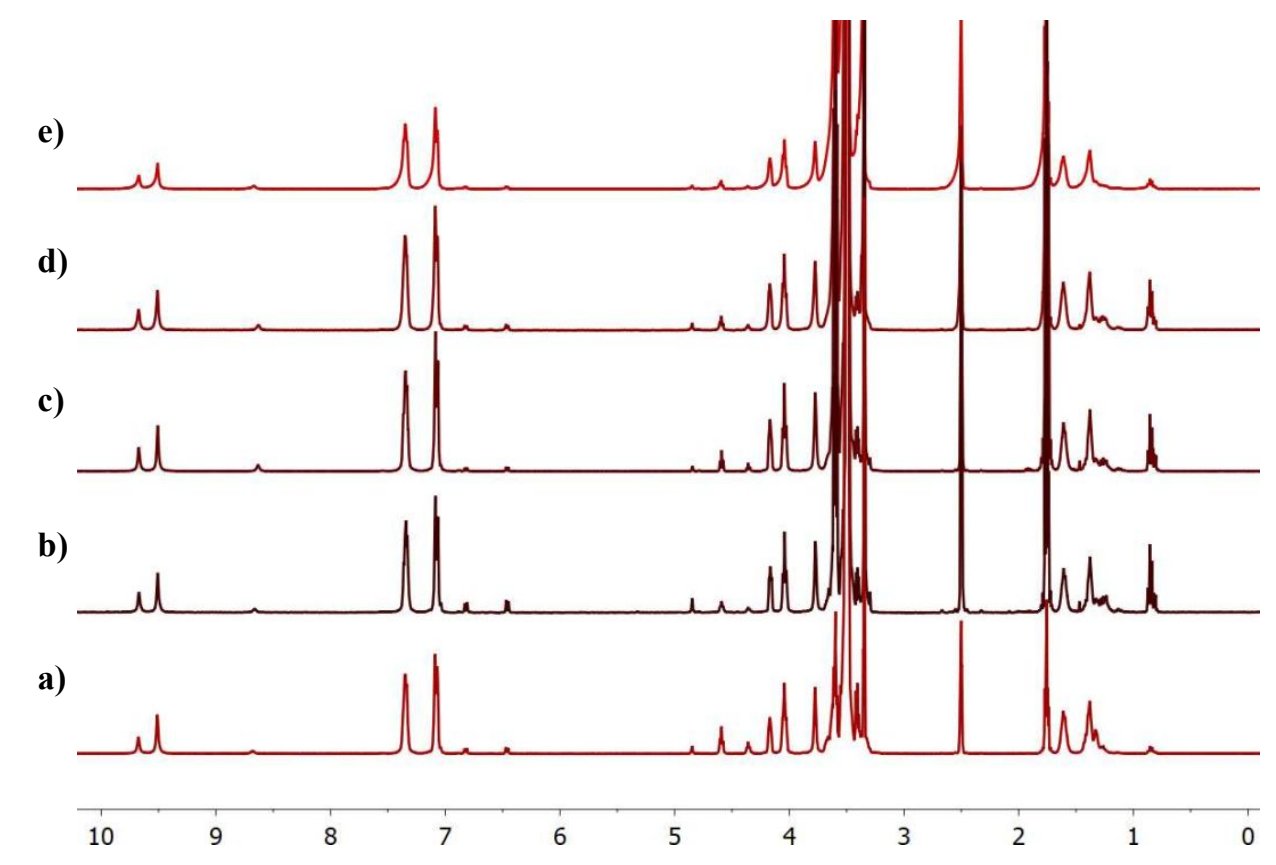

Figure S31: ${ }^{1} \mathrm{H}$ NMR (DMSO- $d_{6}$ ) of FlowPU DBTDL0.1\% at: a) $2 \mathrm{~min}$, b) $5 \mathrm{~min}$, c) $7.5 \mathrm{~min}$, d) $10 \mathrm{~min}$ and e) $20 \mathrm{~min}$. 


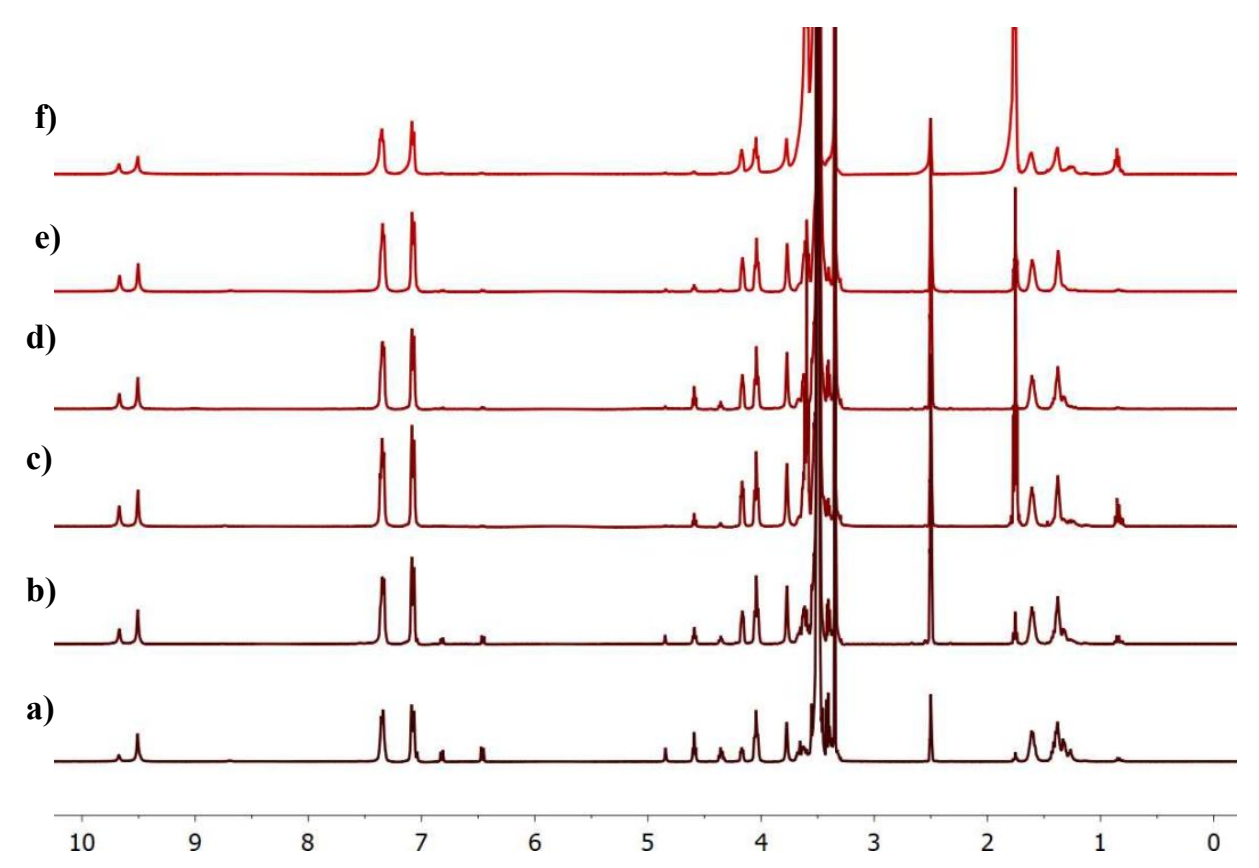

Figure S32: ${ }^{1} \mathrm{H}$ NMR (DMSO- $d_{6}$ ) of FlowPU DBTDL1\% at: a) $\left.30 \mathrm{~s} \mathrm{b)} 60 \mathrm{~s}, \mathrm{c}\right) 120 \mathrm{~s}, \mathrm{~d}$ ) $180 \mathrm{~s}, \mathrm{e}) 240 \mathrm{~s}$ and f) $300 \mathrm{~s}$.

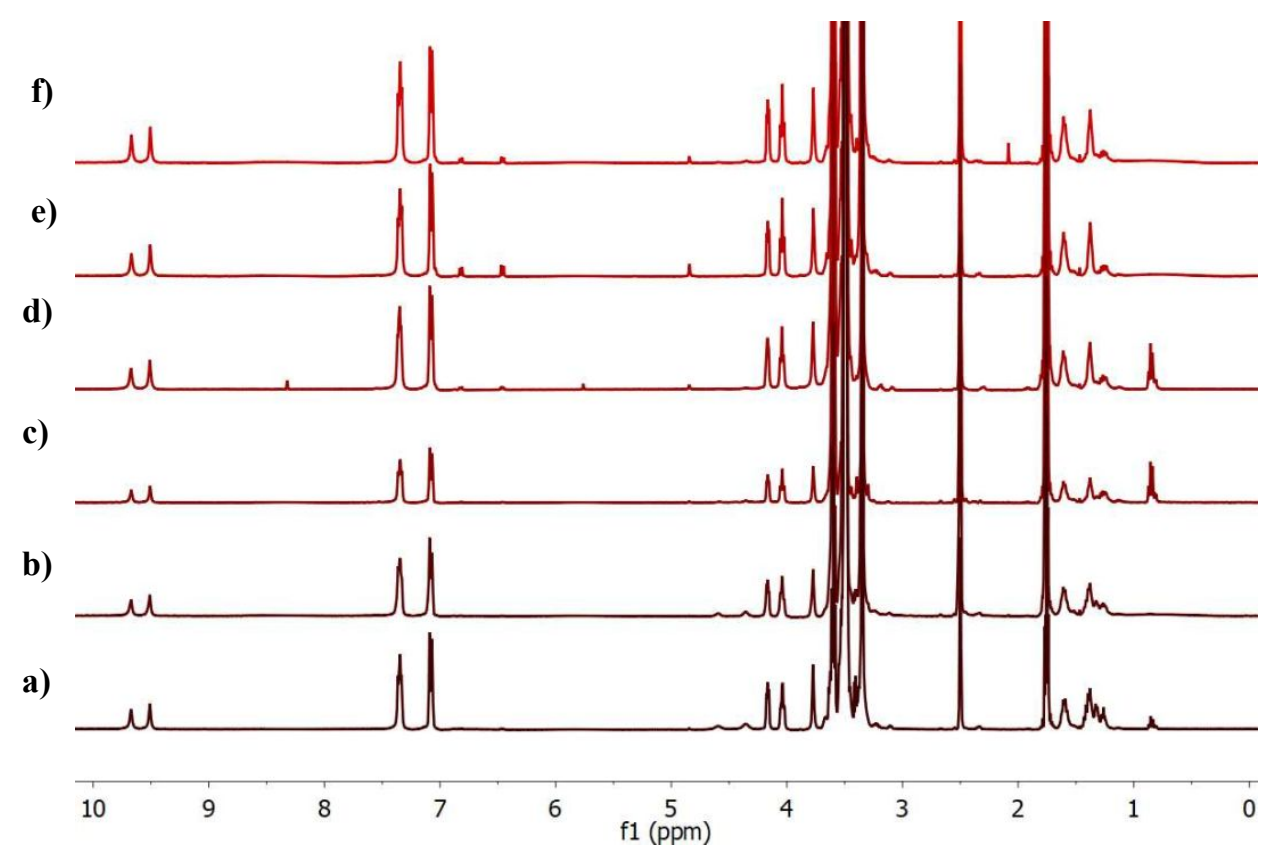

Figure S33: ${ }^{1} \mathrm{H}$ NMR (DMSO- $d_{6}$ ) of FlowPU DBU5 \% at: a) $30 \mathrm{~s} \mathrm{b)} 60 \mathrm{~s}$, c) $120 \mathrm{~s}$, d) $180 \mathrm{~s}$, e) $240 \mathrm{~s}$ and f) $300 \mathrm{~s}$. 


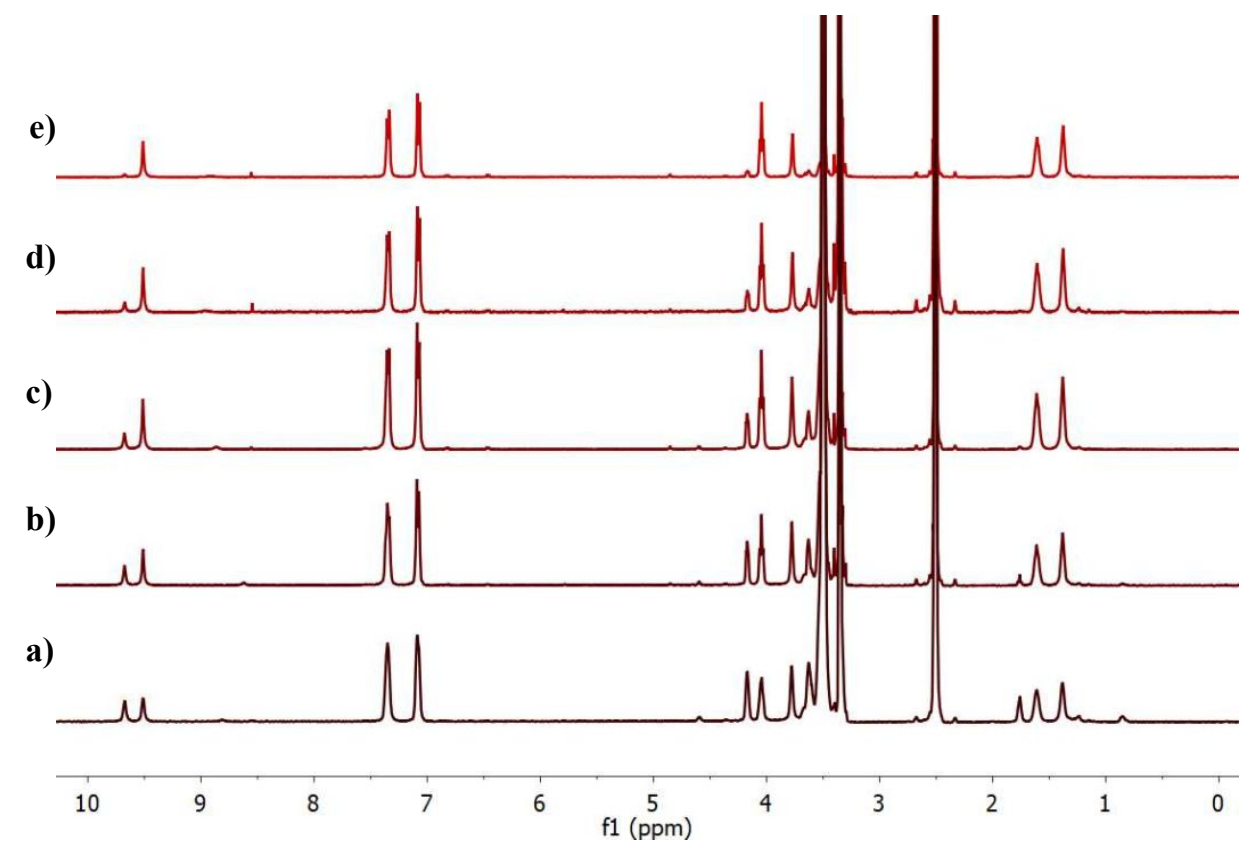

Figure S34: ${ }^{1} \mathrm{H}$ NMR (DMSO- $d_{6}$ ) of PUs varying HS \%: a) FlowPU1, b) FlowPU2, c) FlowPU3, d) FlowPU4 and e) FlowPU5.

d)

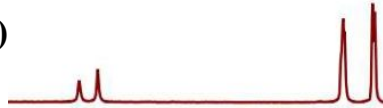

c)

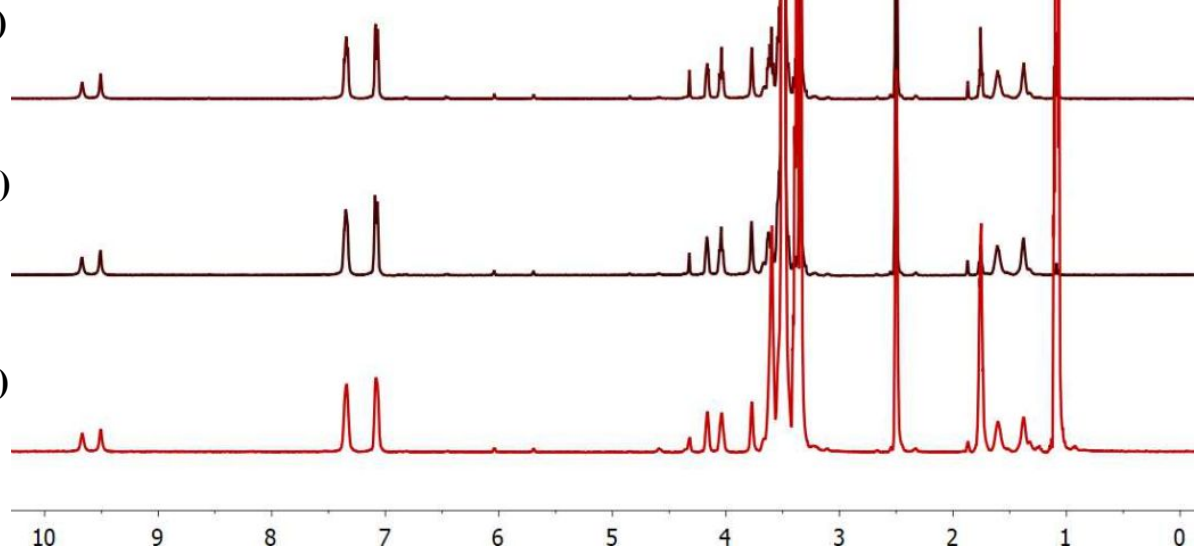

Figure S35: ${ }^{1} \mathrm{H}$ NMR (DMSO- $d_{6}$ ) of FlowPU6 at: a) $120 \mathrm{~s}$, b) $180 \mathrm{~s}$, c) $240 \mathrm{~s}$ and d) $300 \mathrm{~s}$. 


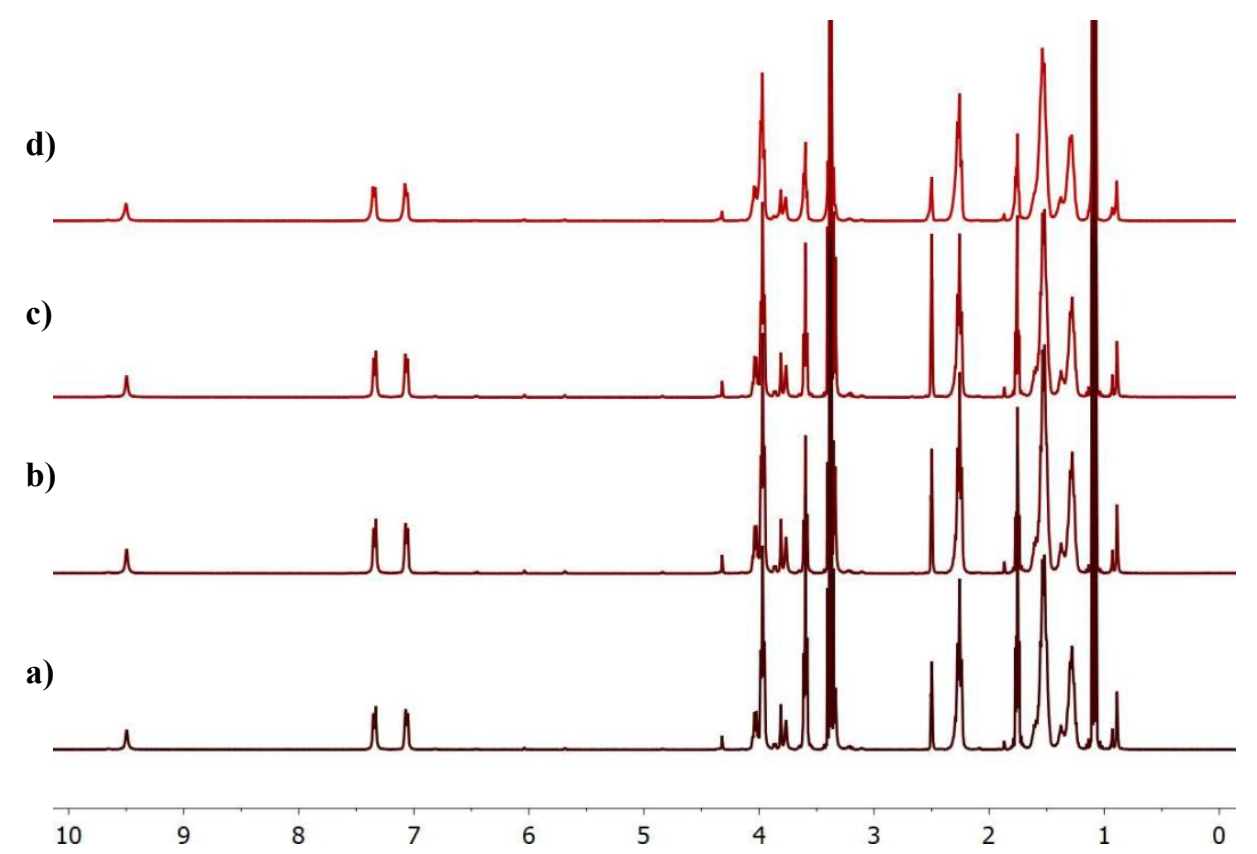

Figure S36: ${ }^{1} \mathrm{H}$ NMR (DMSO- $d_{6}$ ) of FlowPU7 at: a) $120 \mathrm{~s}$, b) $\left.180 \mathrm{~s}, \mathrm{c}\right) 240 \mathrm{~s}$ and d) $300 \mathrm{~s}$.

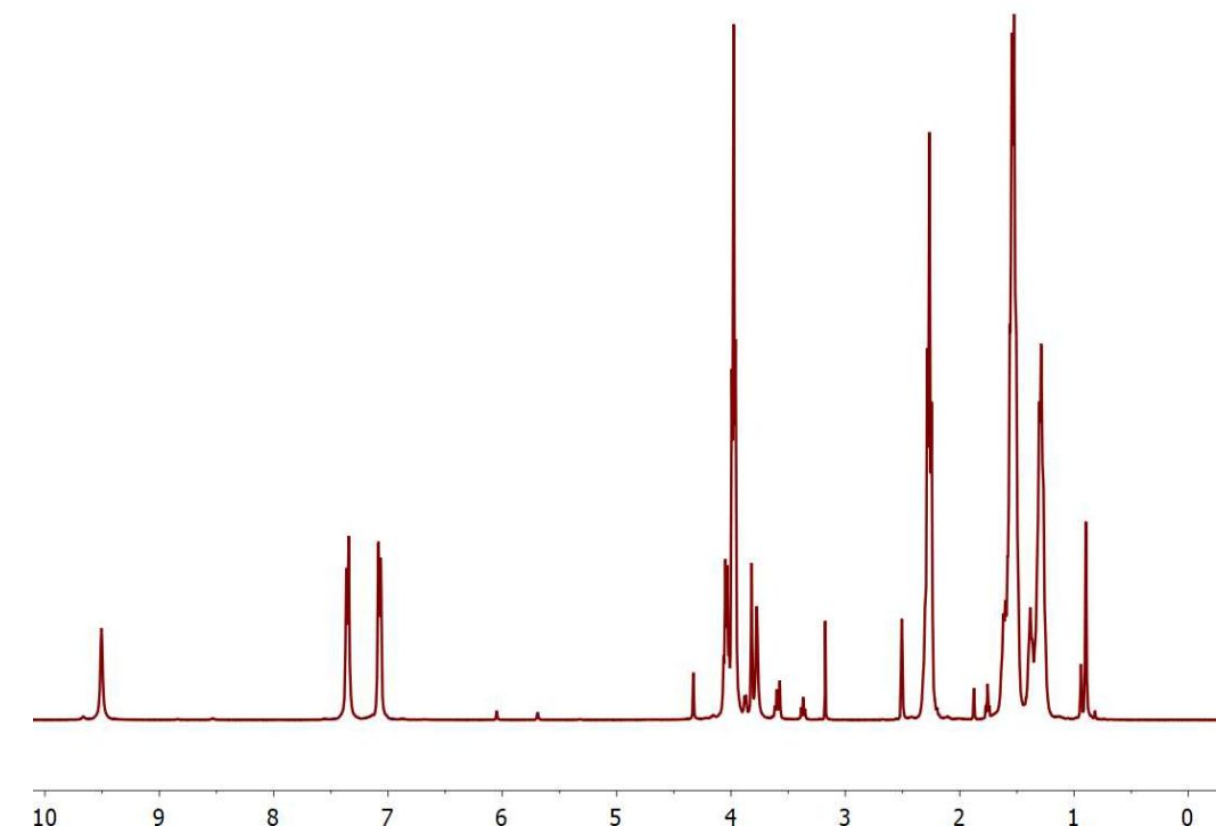

Figure S37: ${ }^{1} \mathrm{H}$ NMR (DMSO- $d_{6}$ ) of FlowPU8 after $210 \mathrm{~s}$. 


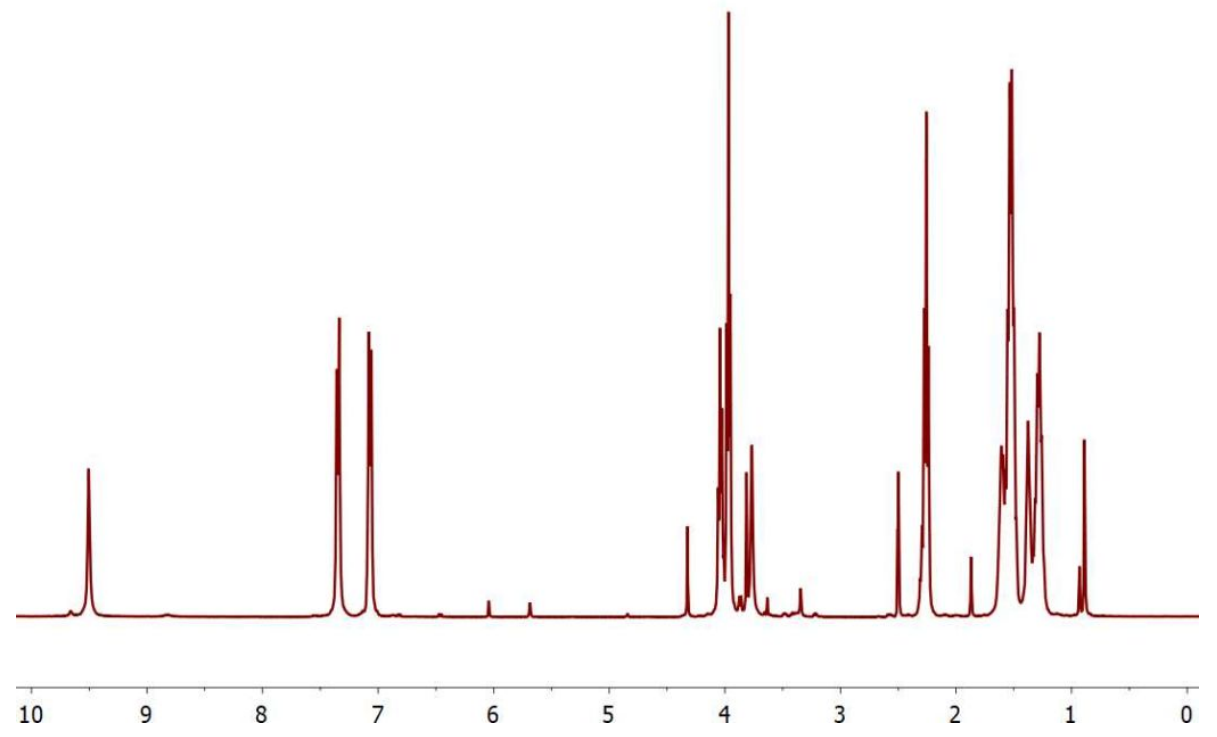

Figure S38: ${ }^{1} \mathrm{H}$ NMR (DMSO- $d_{6}$ ) of FlowPU9 after $210 \mathrm{~s}$.

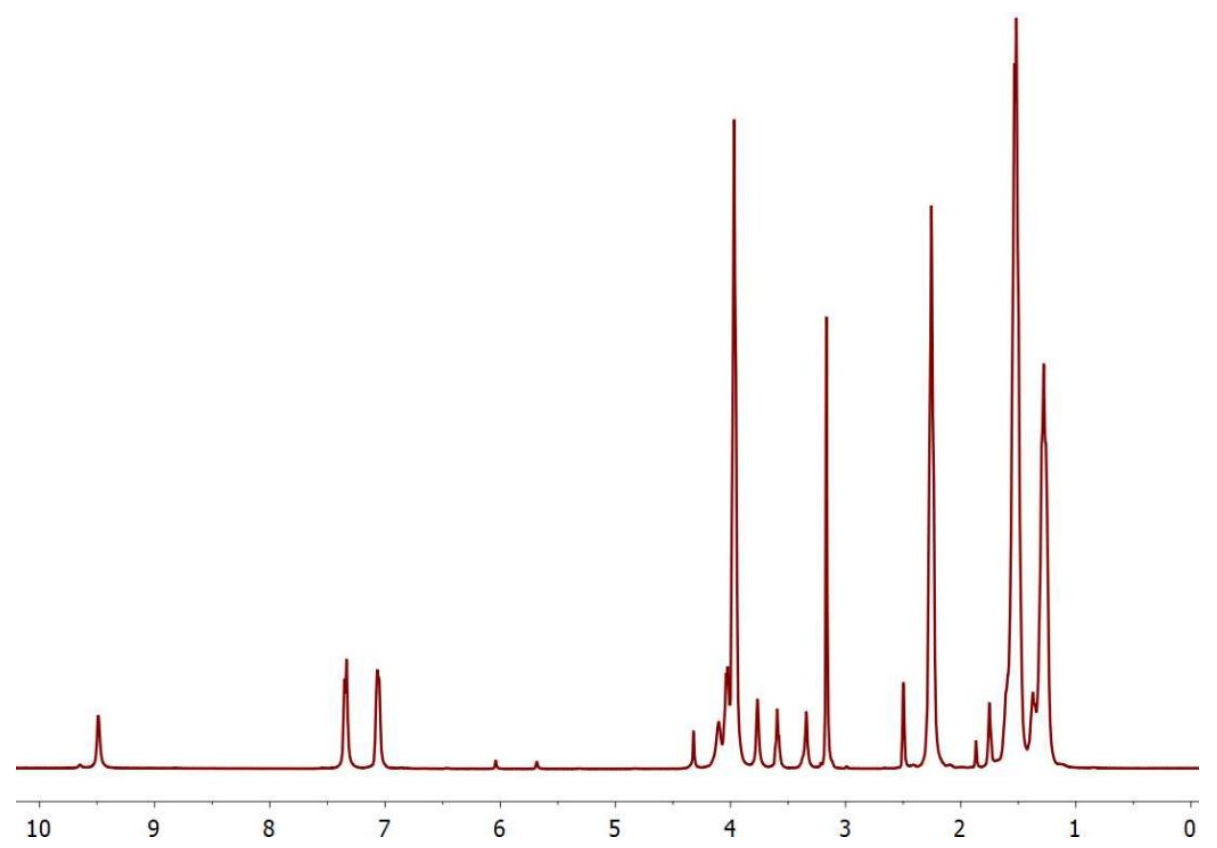

Figure S39: ${ }^{1} \mathrm{H}$ NMR (DMSO- $\left.d_{6}\right)$ of FlowPU10 after $240 \mathrm{~s}$. 


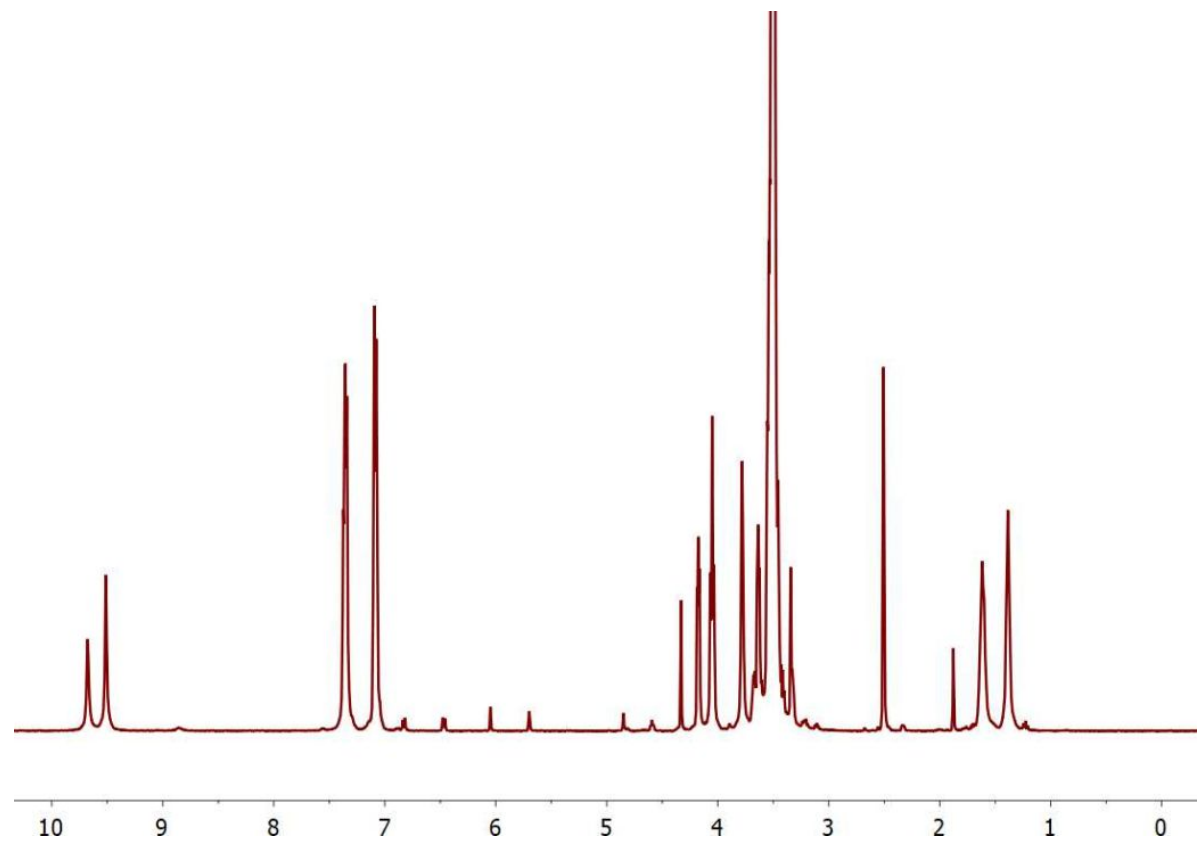

Figure S40: ${ }^{1} \mathrm{H}$ NMR (DMSO- $\left.d_{6}\right)$ of FlowPU11 after $300 \mathrm{~s}$.

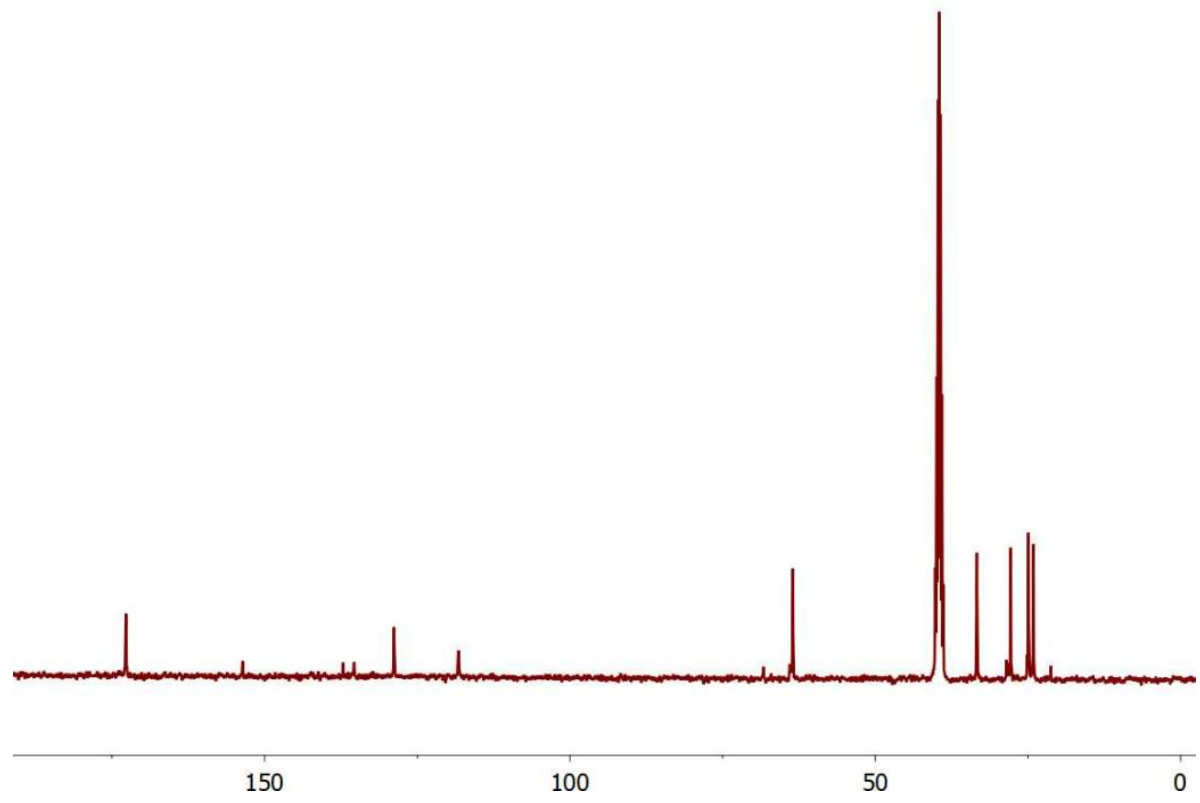

Figure S41: ${ }^{13} \mathrm{C}$ NMR (DMSO- $d_{6}$ ) of FlowPU8 after $210 \mathrm{~s}$. 


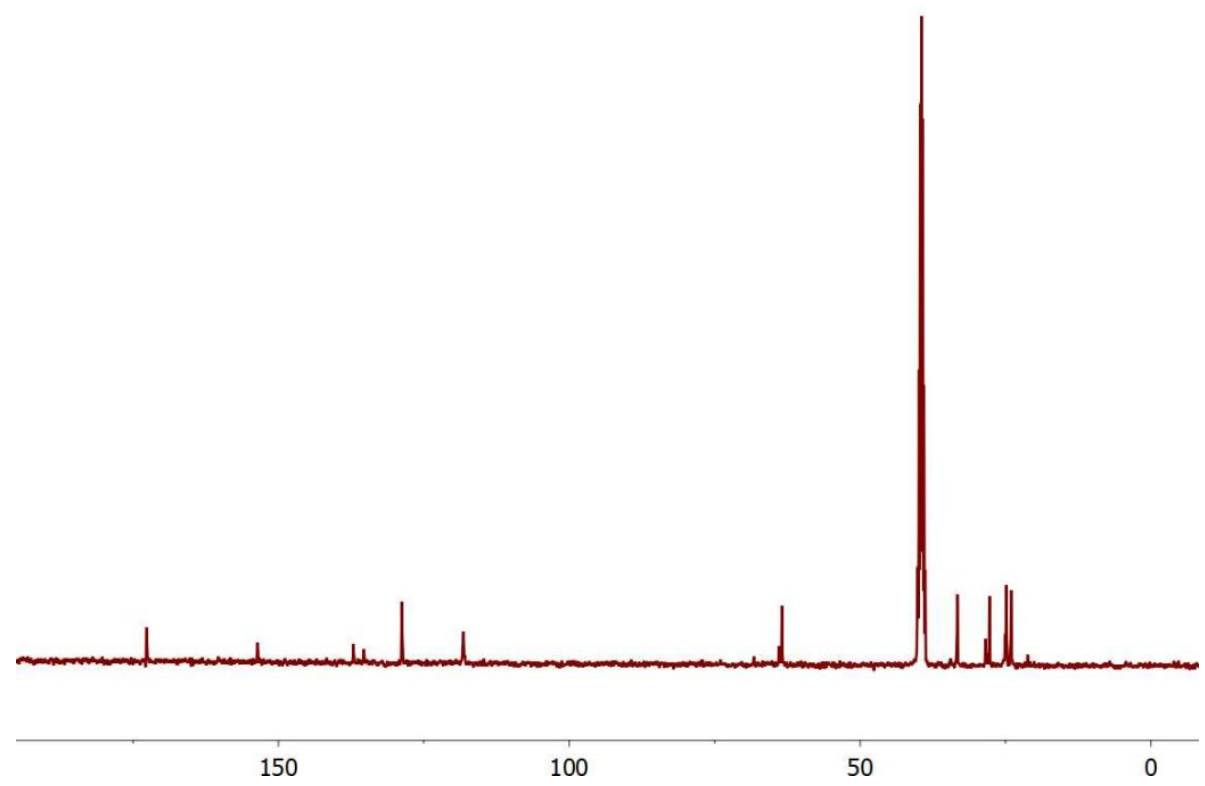

Figure S42: ${ }^{13} \mathrm{C}$ NMR (DMSO- $d_{6}$ ) of FlowPU9 after $210 \mathrm{~s}$.

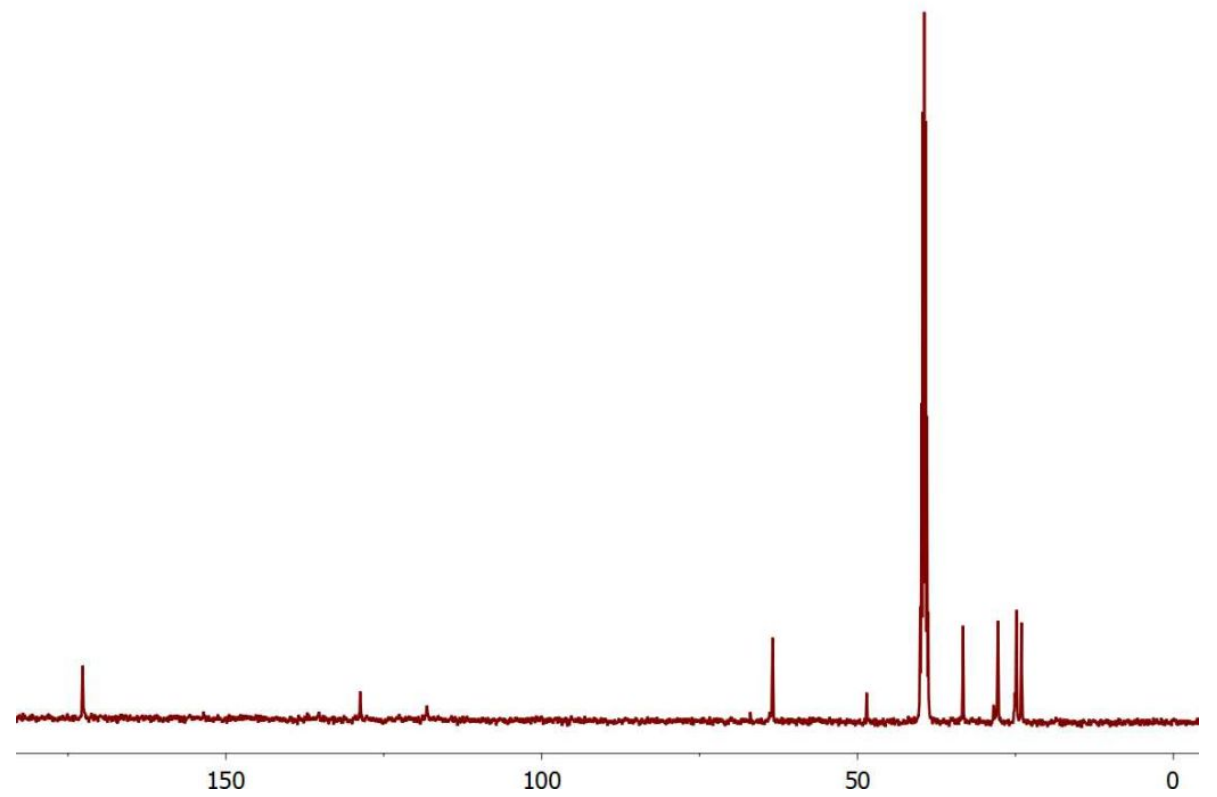

Figure S43: ${ }^{13} \mathrm{C}$ NMR (DMSO- $\left.d_{6}\right)$ of FlowPU10 after $240 \mathrm{~s}$. 


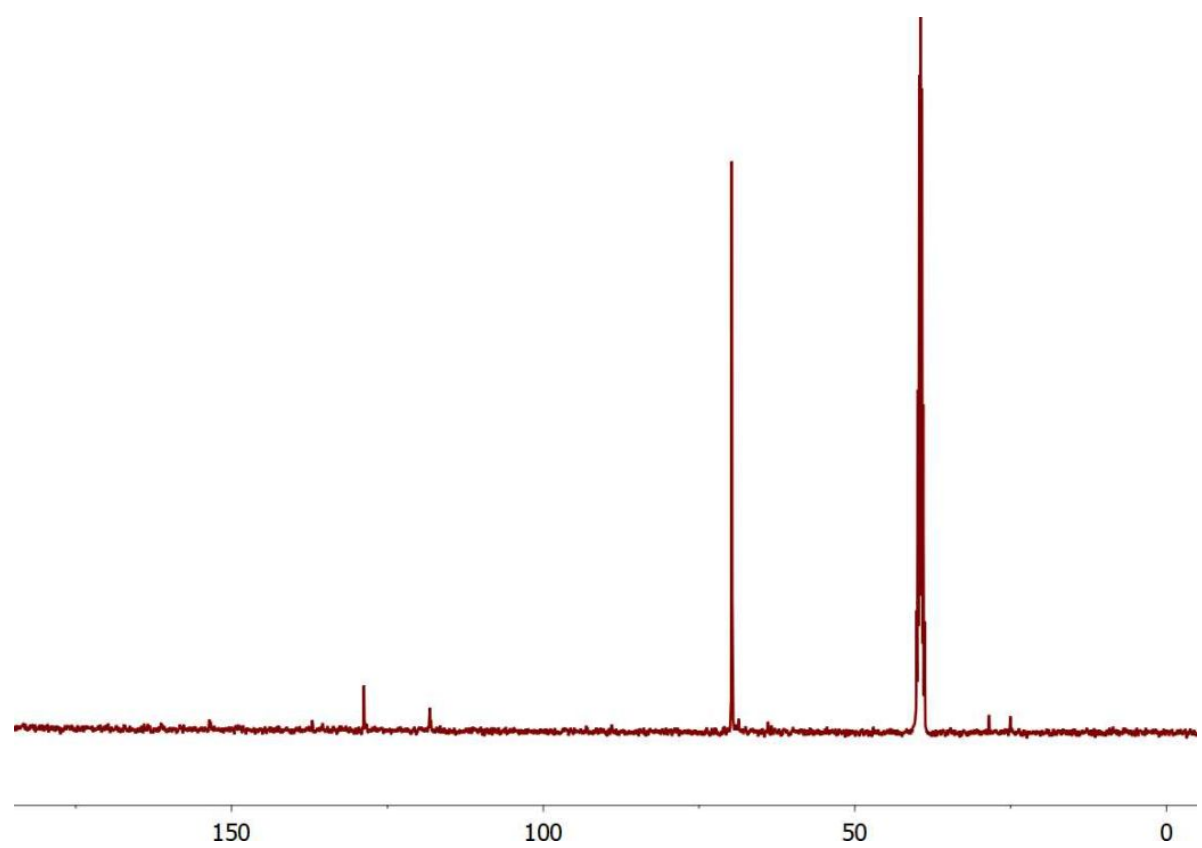

Figure S44: ${ }^{13} \mathrm{C}$ NMR (DMSO- $d_{6}$ ) of FlowPU11 after $300 \mathrm{~s}$.

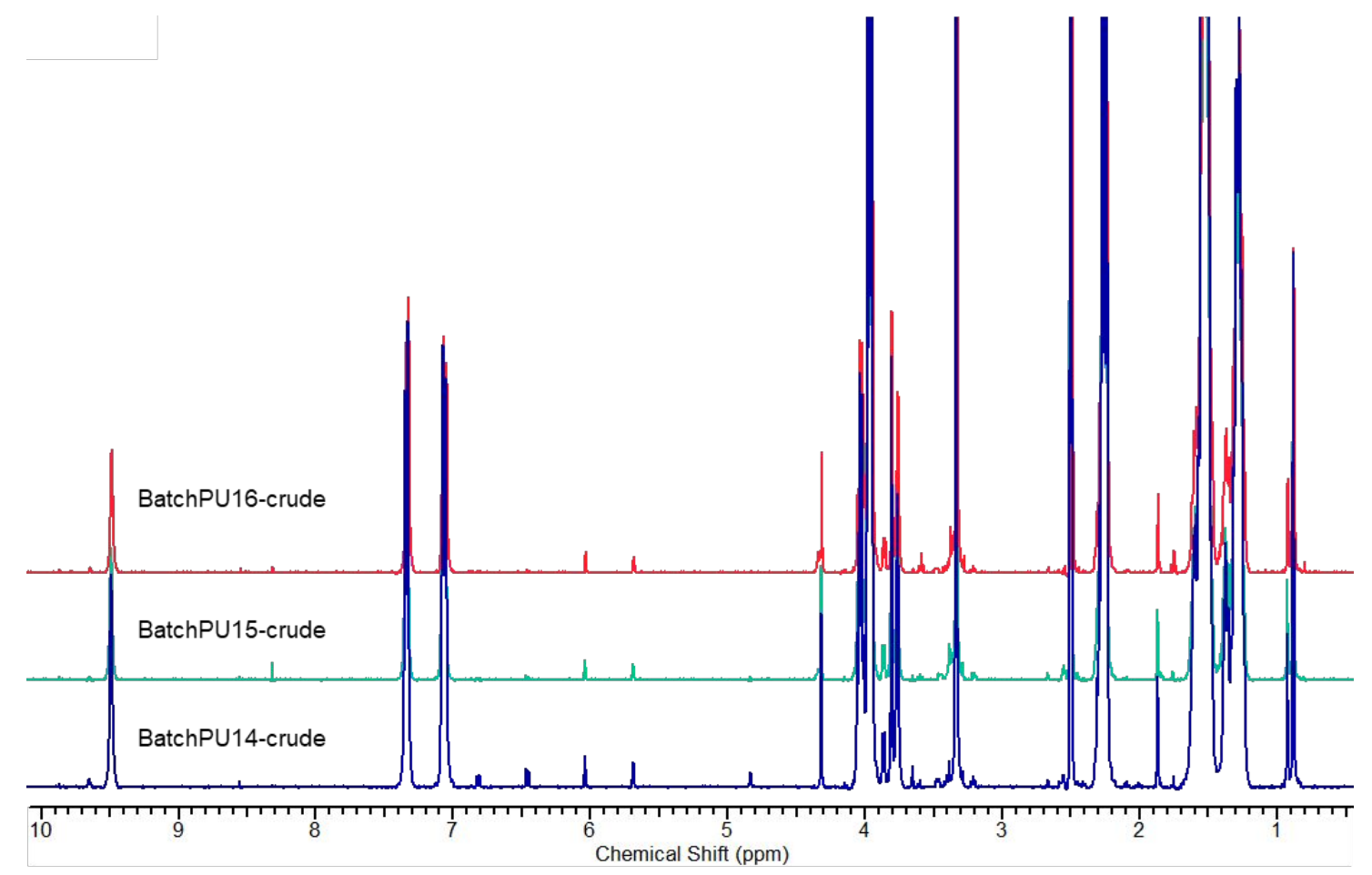

Figure S45: ${ }^{1} \mathrm{H}$ NMR (DMSO- $d_{6}$ ) of crude material from large-scale batch polymerizations. 


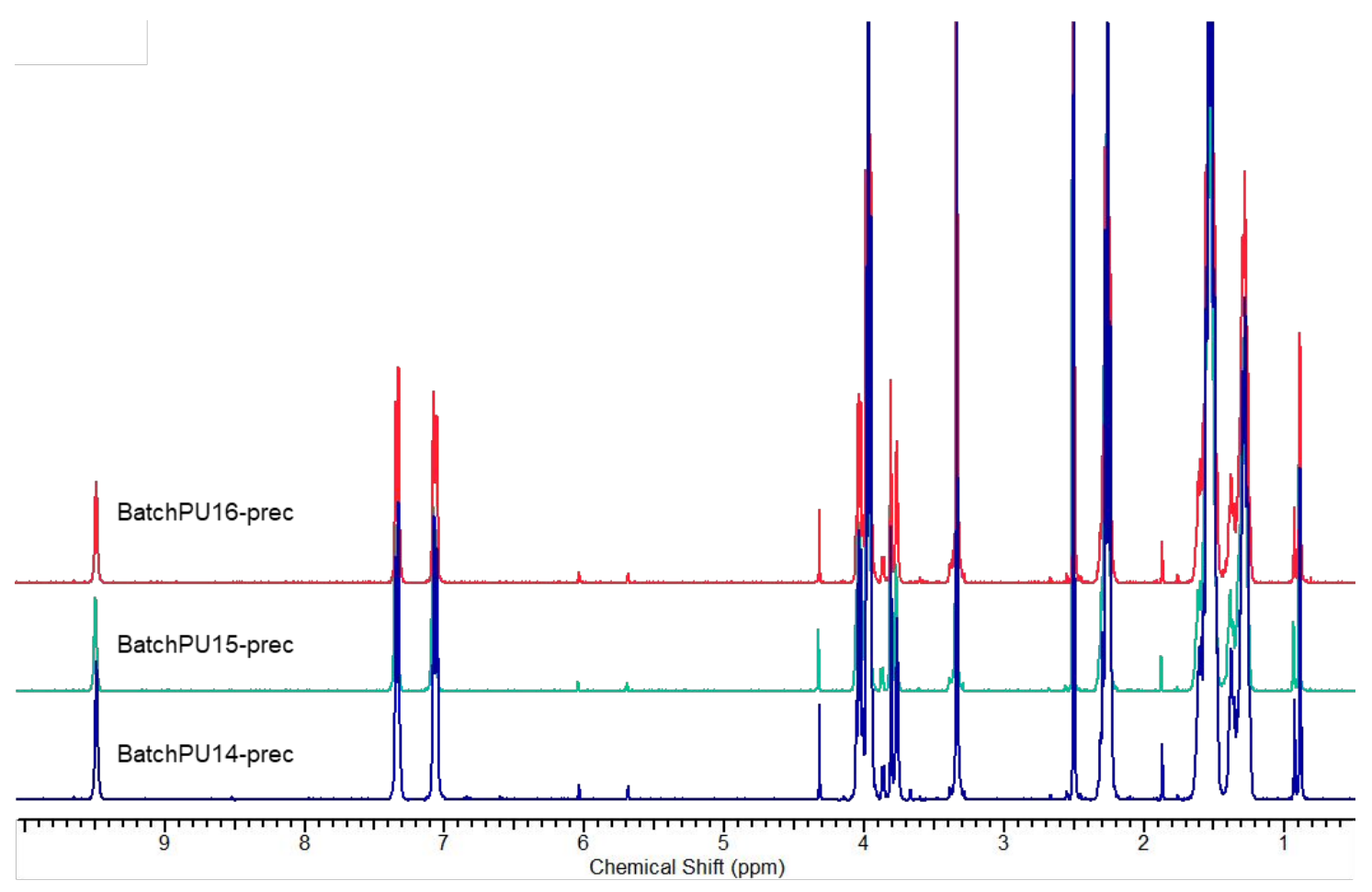

Figure S46: ${ }^{1} \mathrm{H}$ NMR (DMSO- $\left.d_{6}\right)$ of purified PUs from large-scale batch polymerizations.

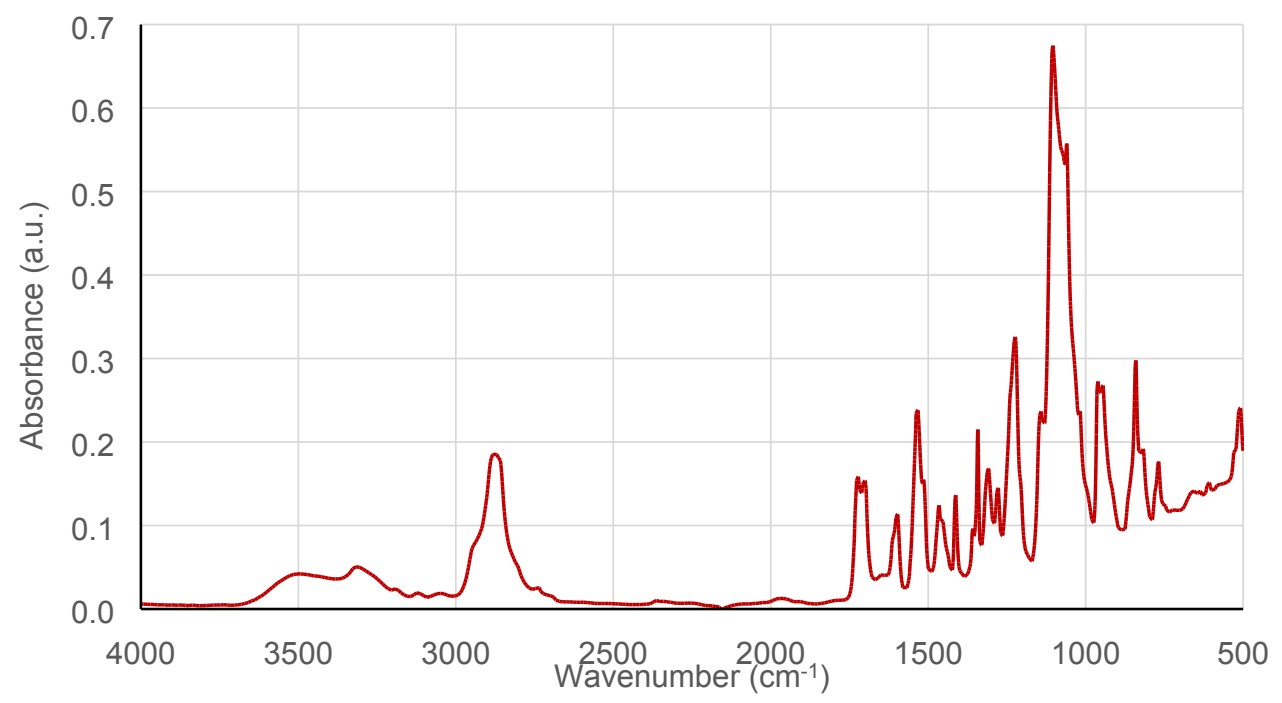

Figure S47: FTIR spectrum of BatchPU1. 


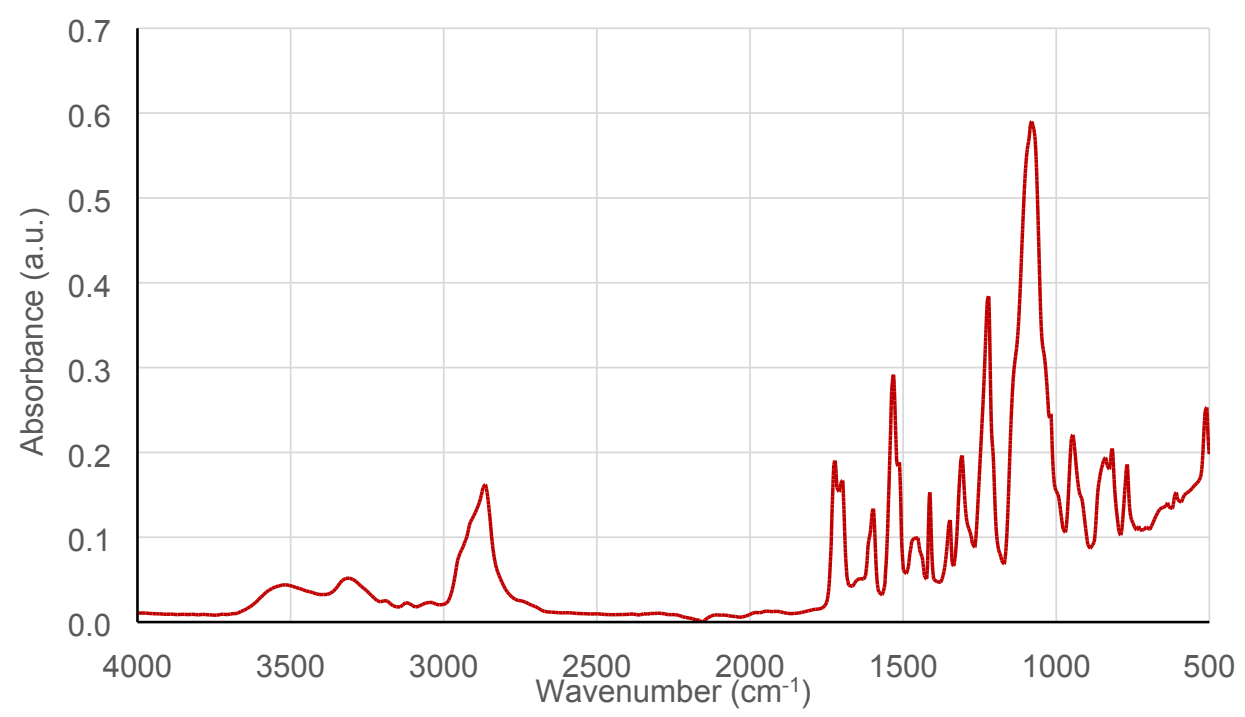

Figure S48: FTIR spectrum of BatchPU2.

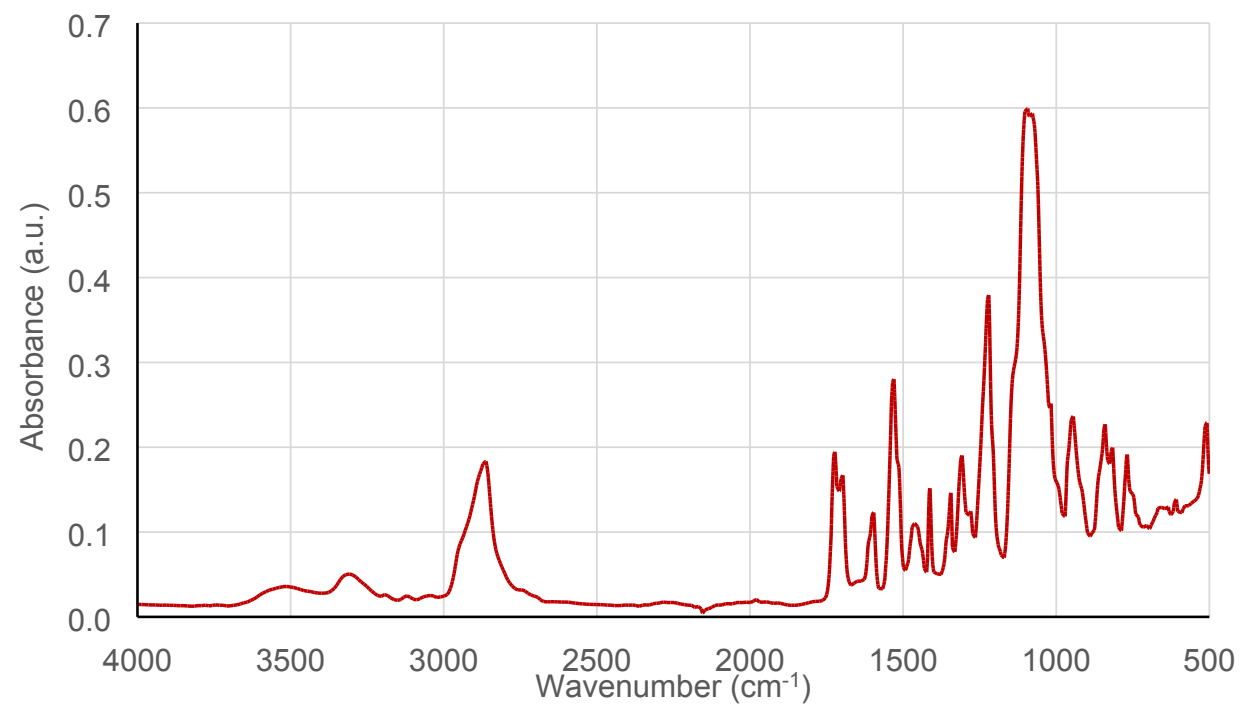

Figure S49: FTIR spectrum of BatchPU3. 


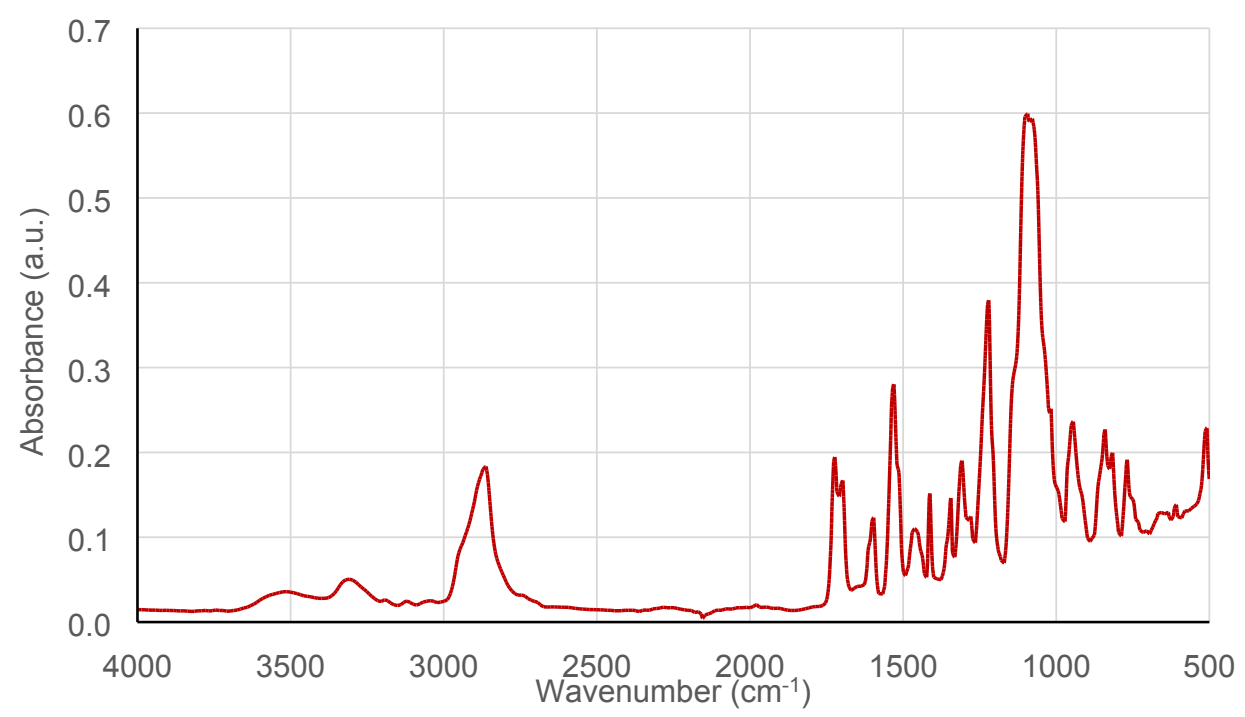

Figure S50: FTIR spectrum of BatchPU4.

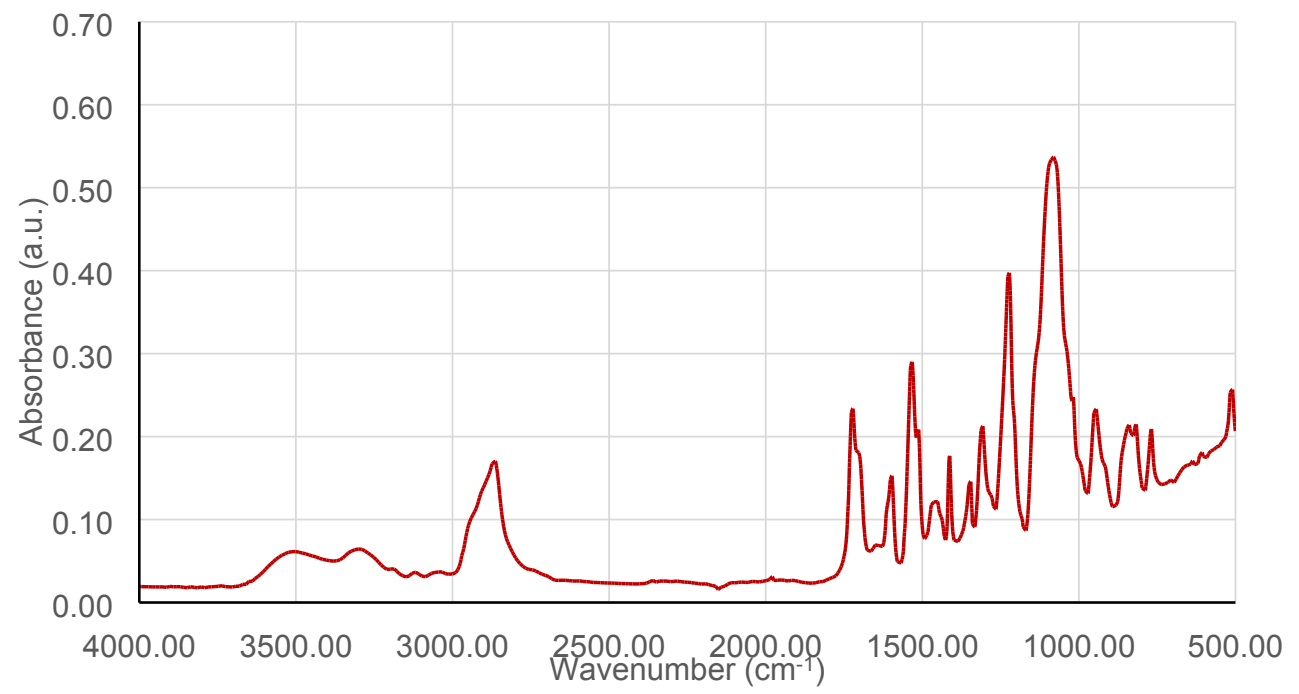

Figure S51: FTIR spectrum of BatchPU5. 


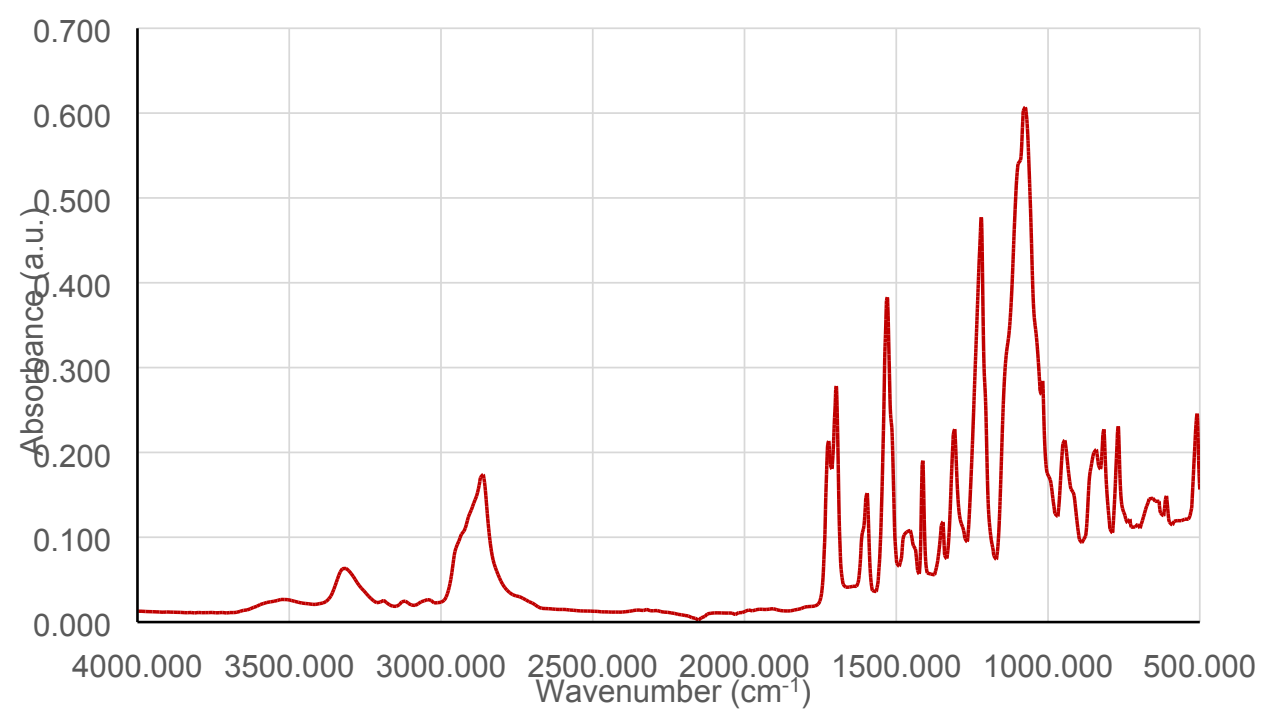

Figure S52: FTIR spectrum of BatchPU6.

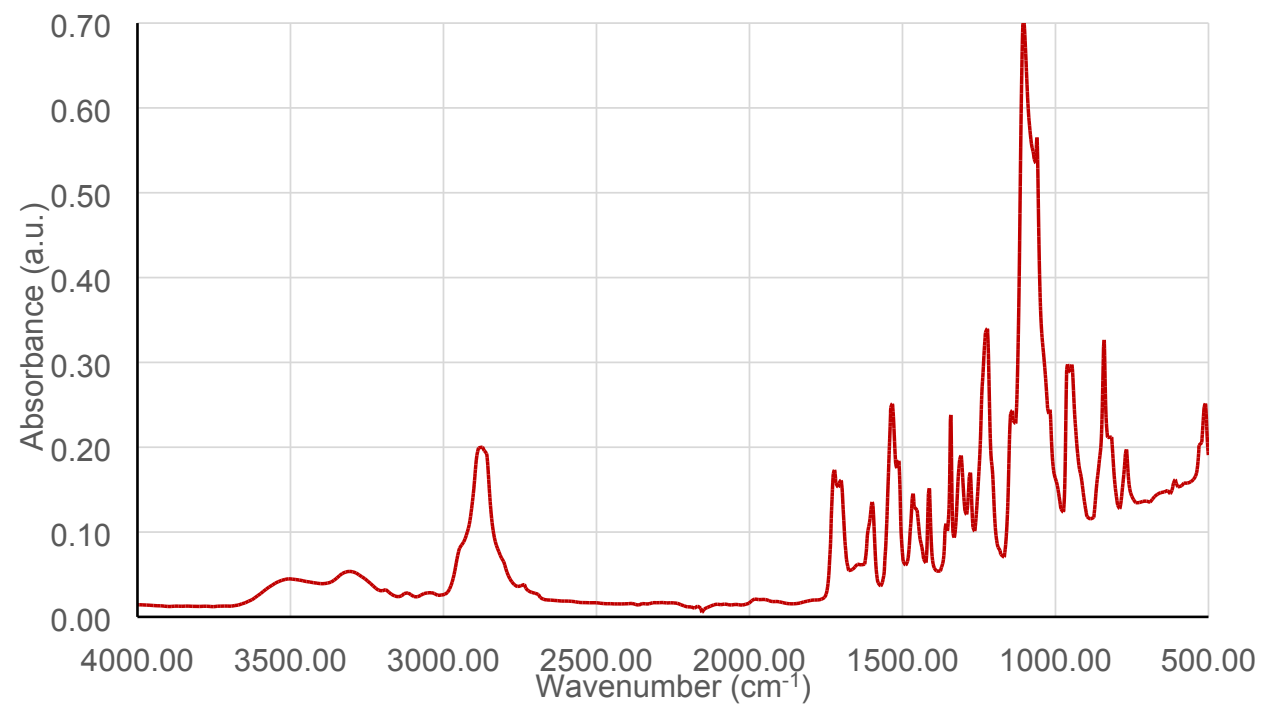

Figure S53: FTIR spectrum of BatchPU7. 


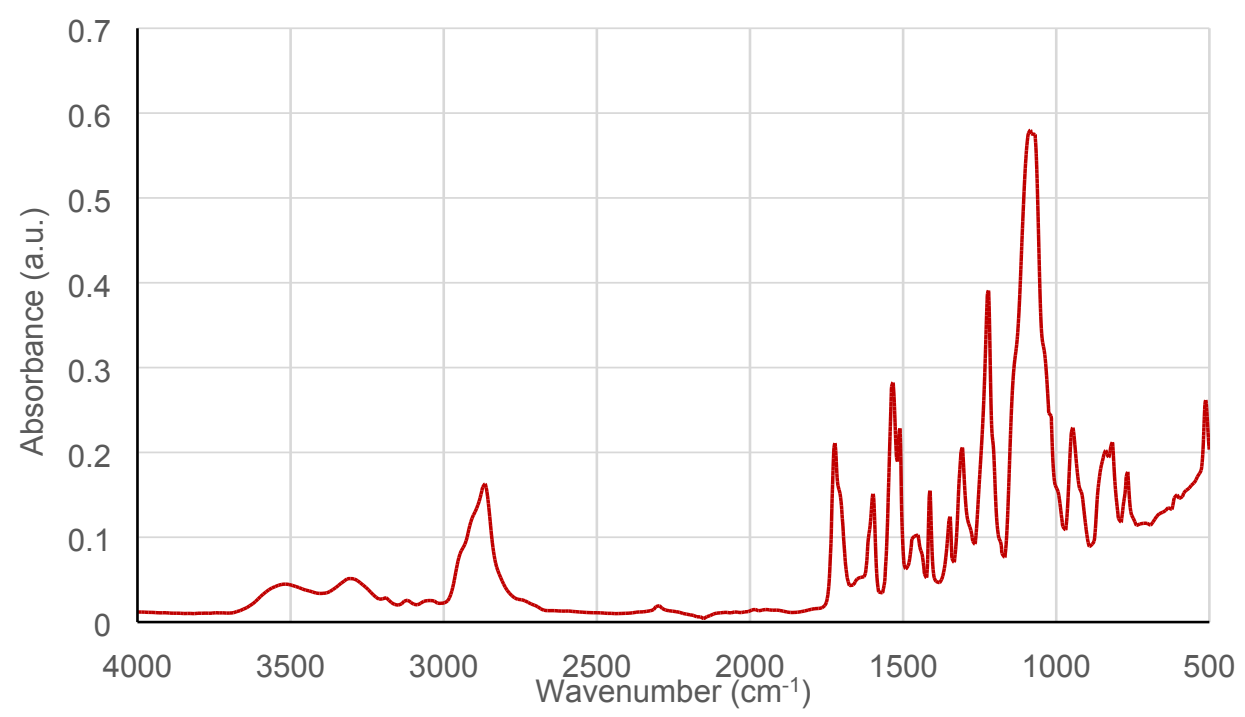

Figure S54: FTIR spectrum of BatchPU8.

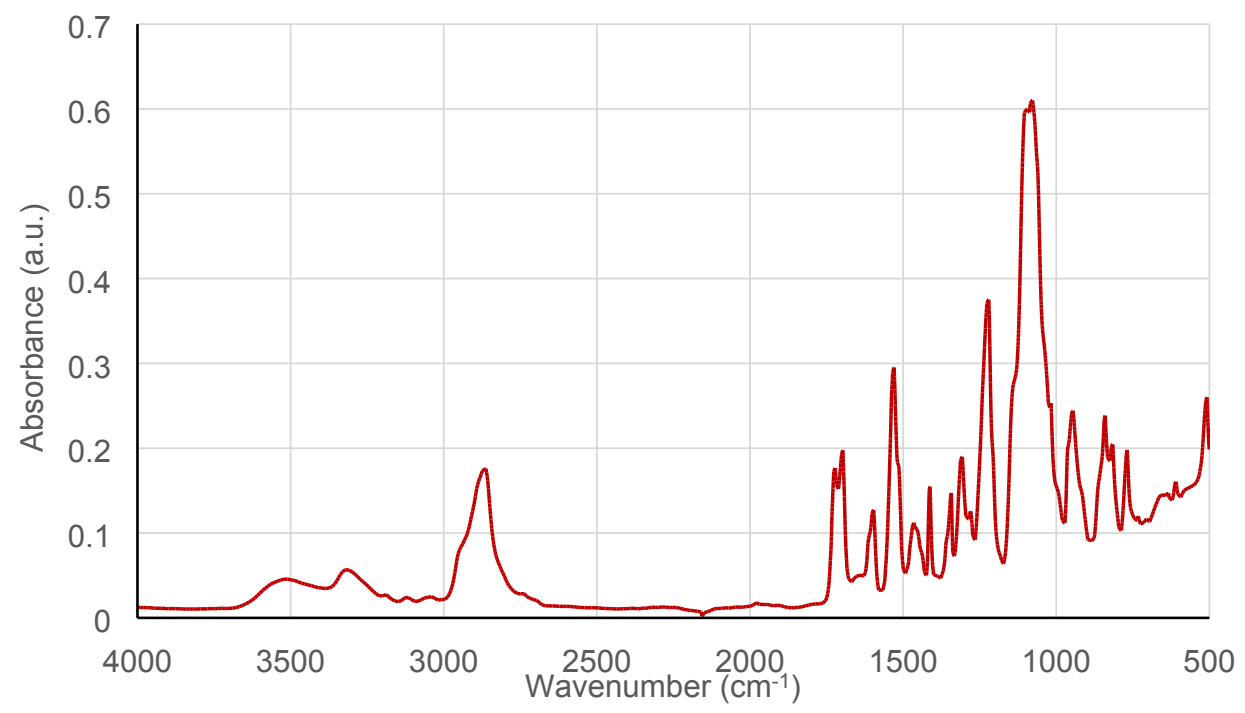

Figure S55: FTIR spectrum of BatchPU9. 


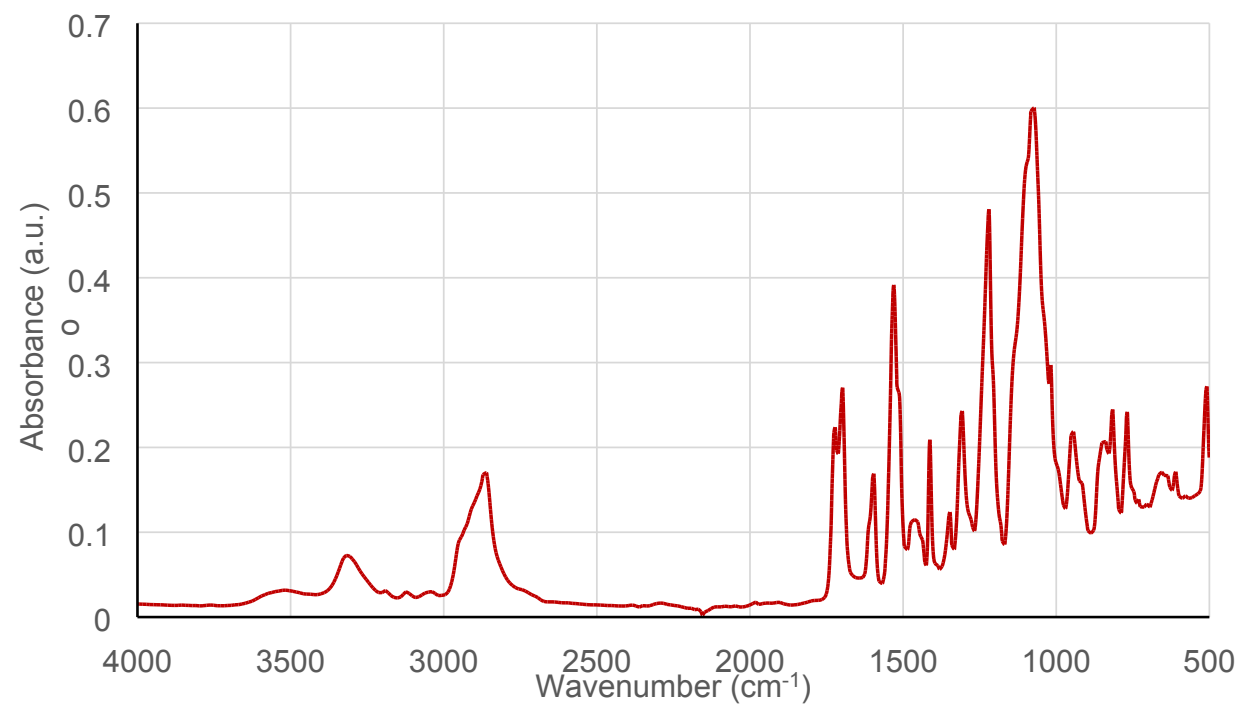

Figure S56: FTIR spectrum of BatchPU10.

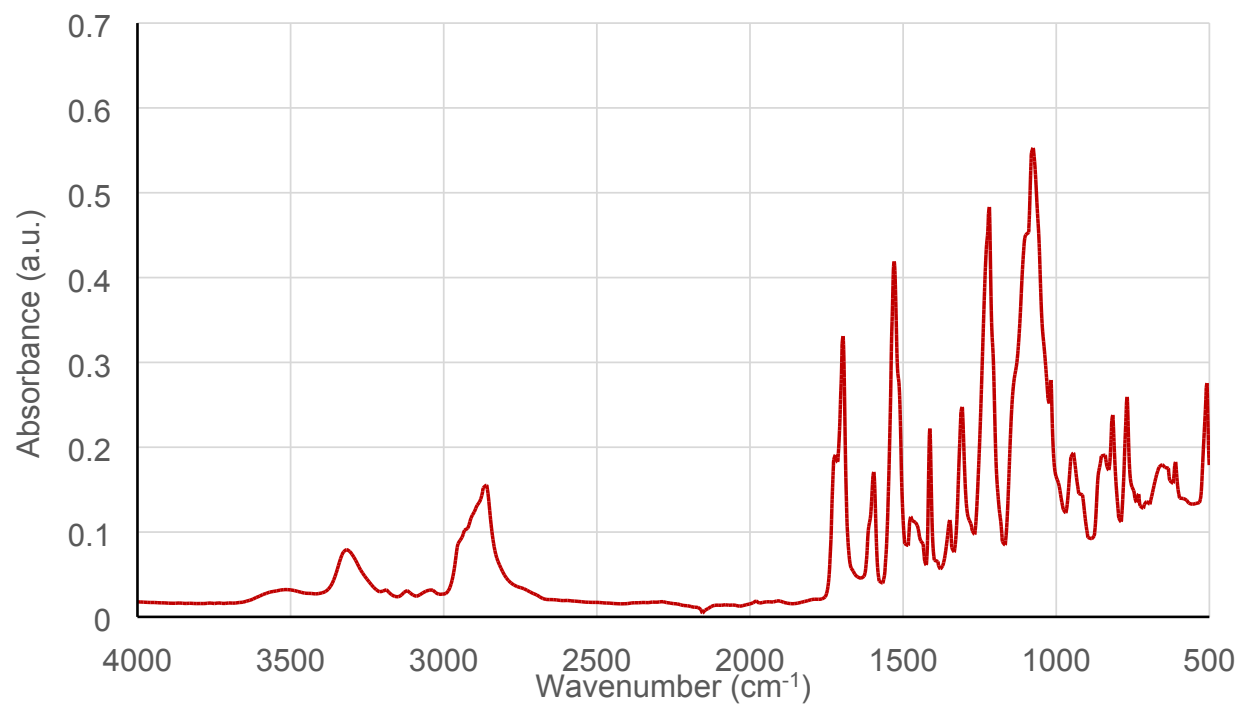

Figure S57: FTIR spectrum of BatchPU11. 


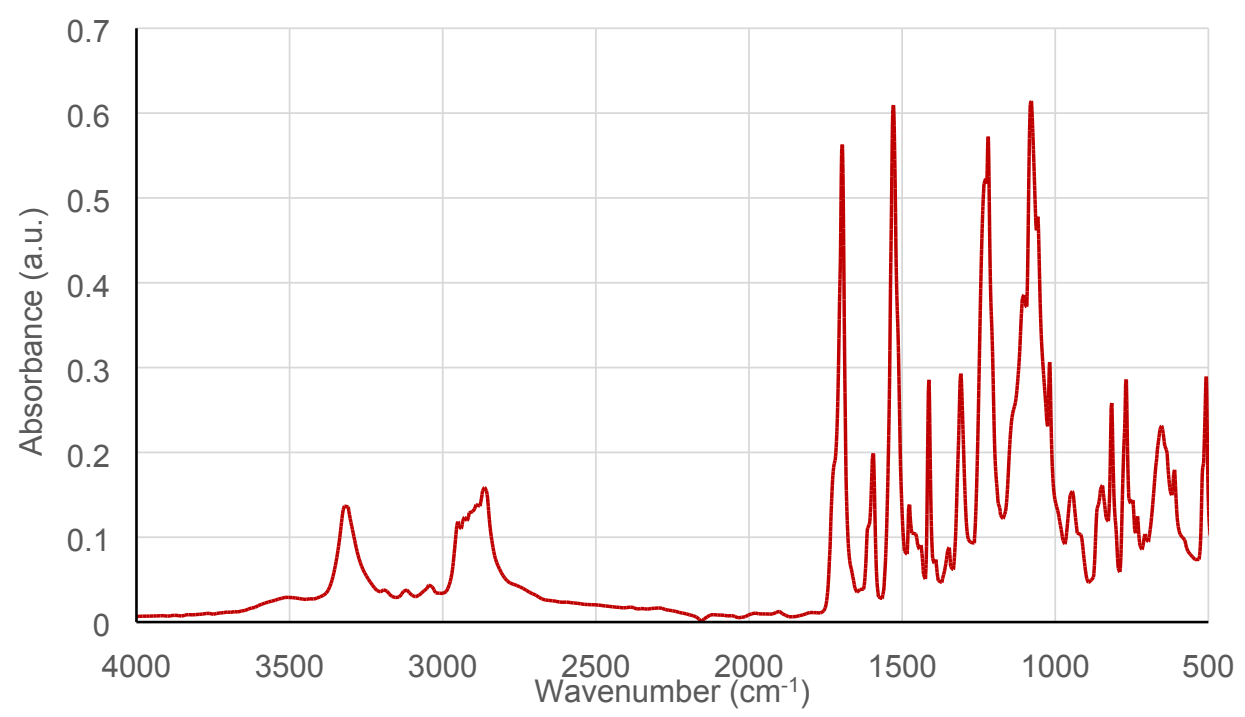

Figure S58: FTIR spectrum of BatchPU12.

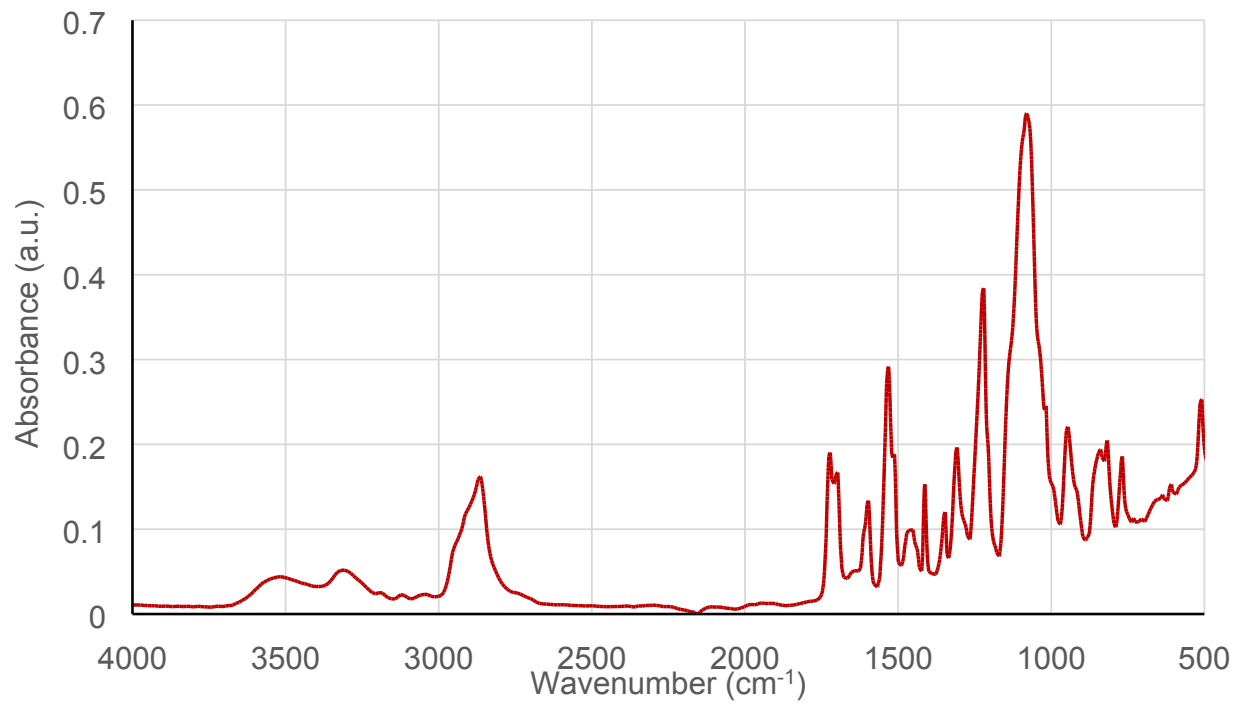

Figure S59: FTIR spectrum of BatchPU13. 


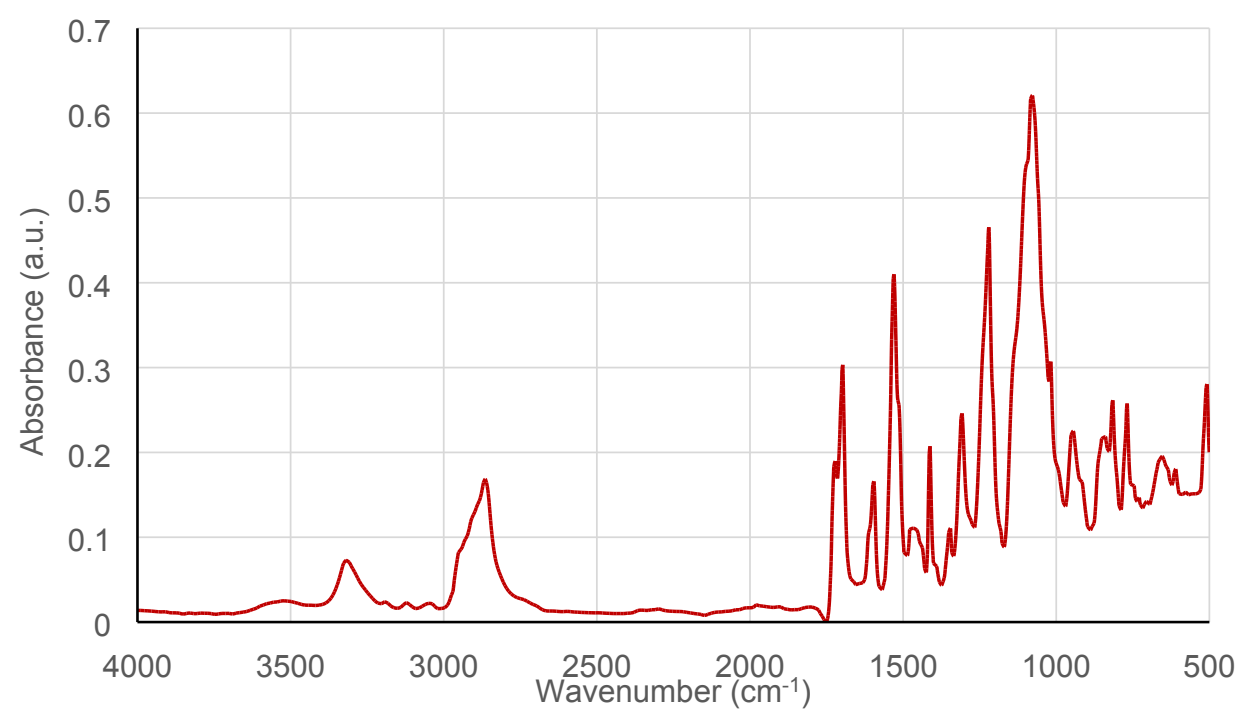

Figure S60: FTIR spectrum of FlowPUDBTDL0.1\% after 20 min reaction time.

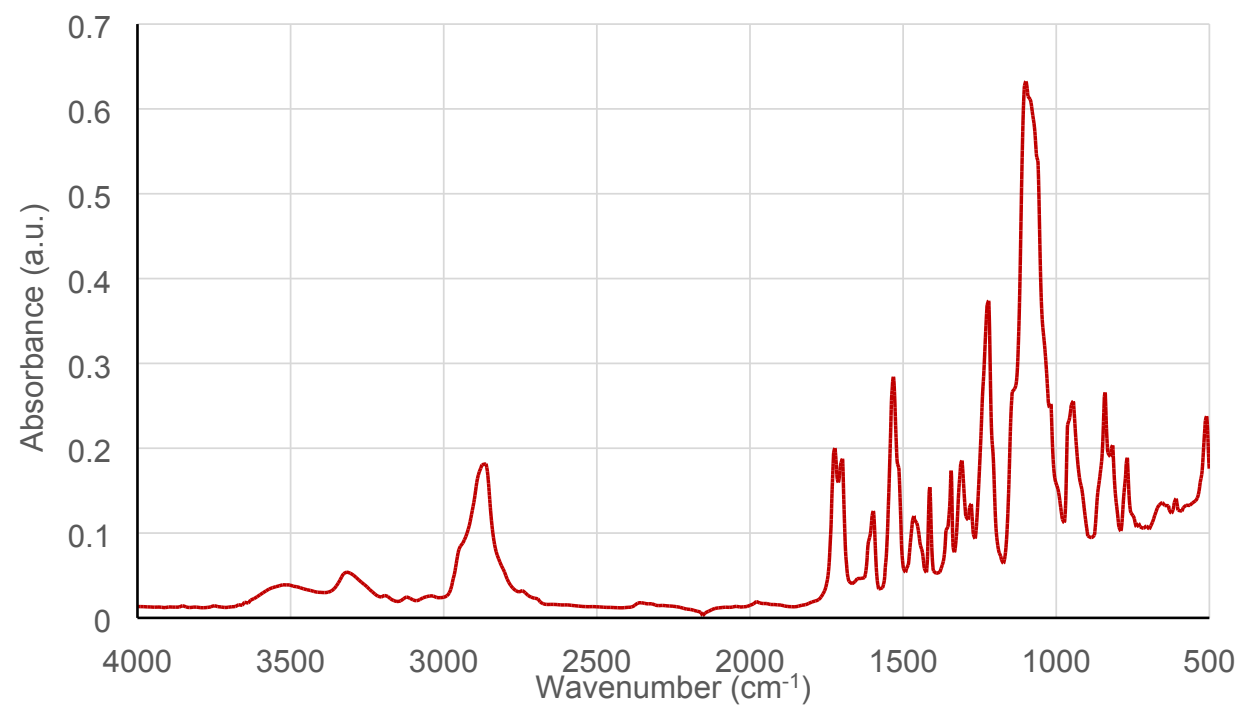

Figure S61: FTIR spectrum of FlowPUDBTDL1\% after $300 \mathrm{~s}$ reaction time. 


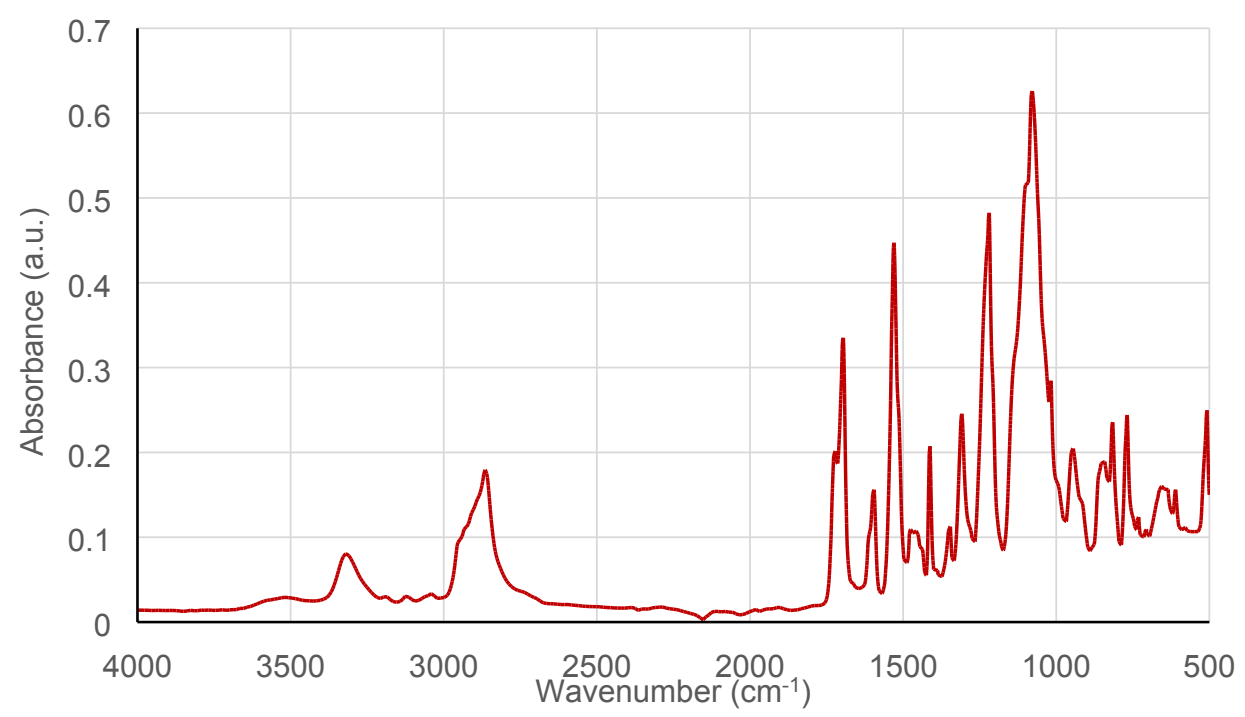

Figure S62: FTIR spectrum of FlowPUDBU5\% after 300 s reaction time.

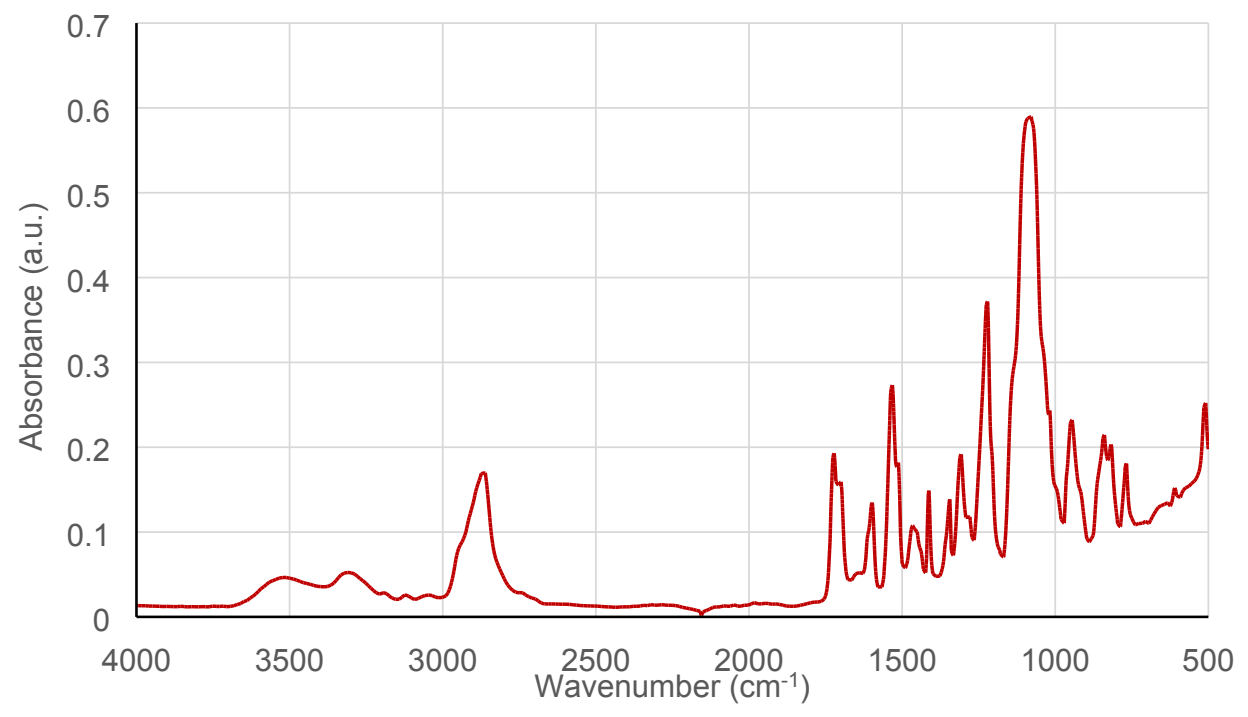

Figure S63: FTIR spectrum of FlowPU1. 


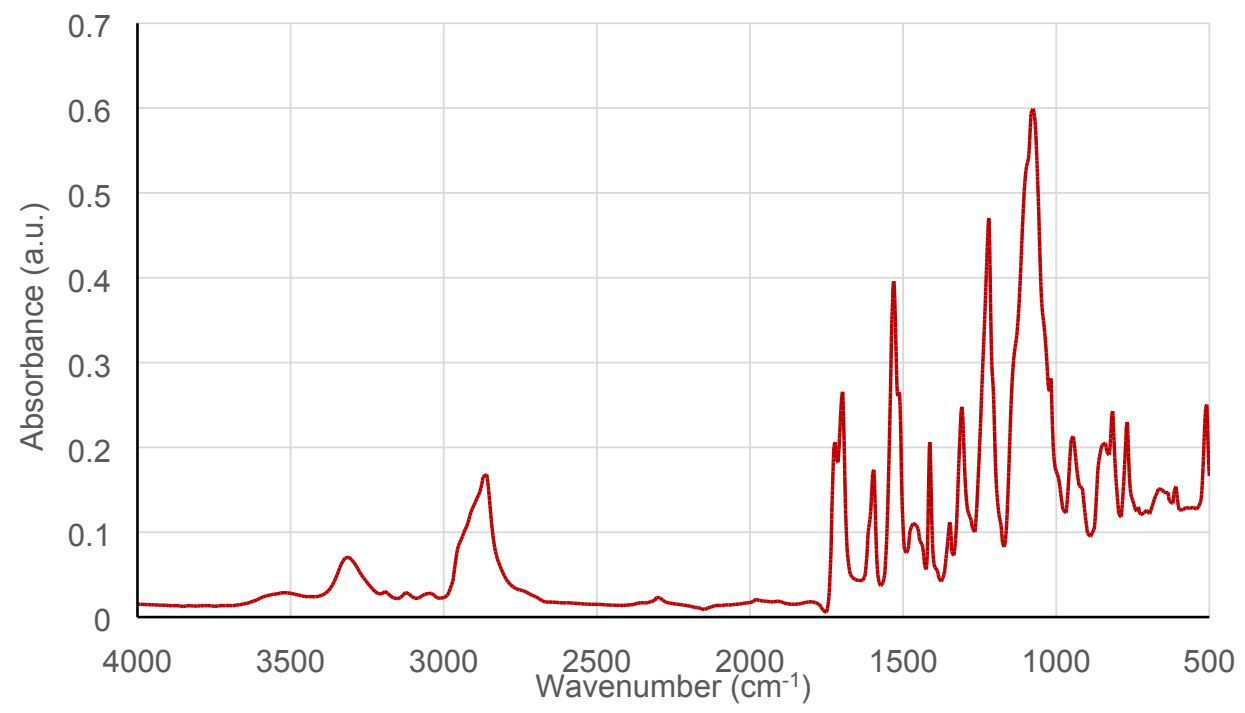

Figure S64: FTIR spectrum of FlowPU2.

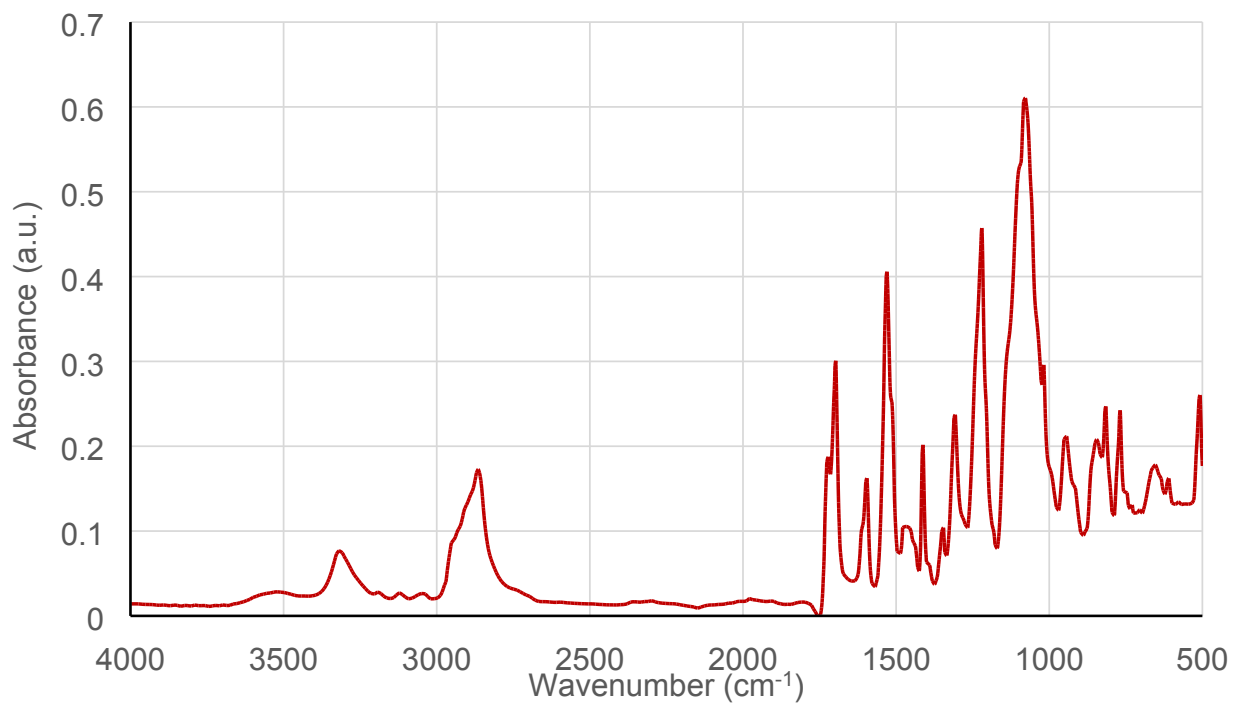

Figure S65: FTIR spectrum of FlowPU3. 


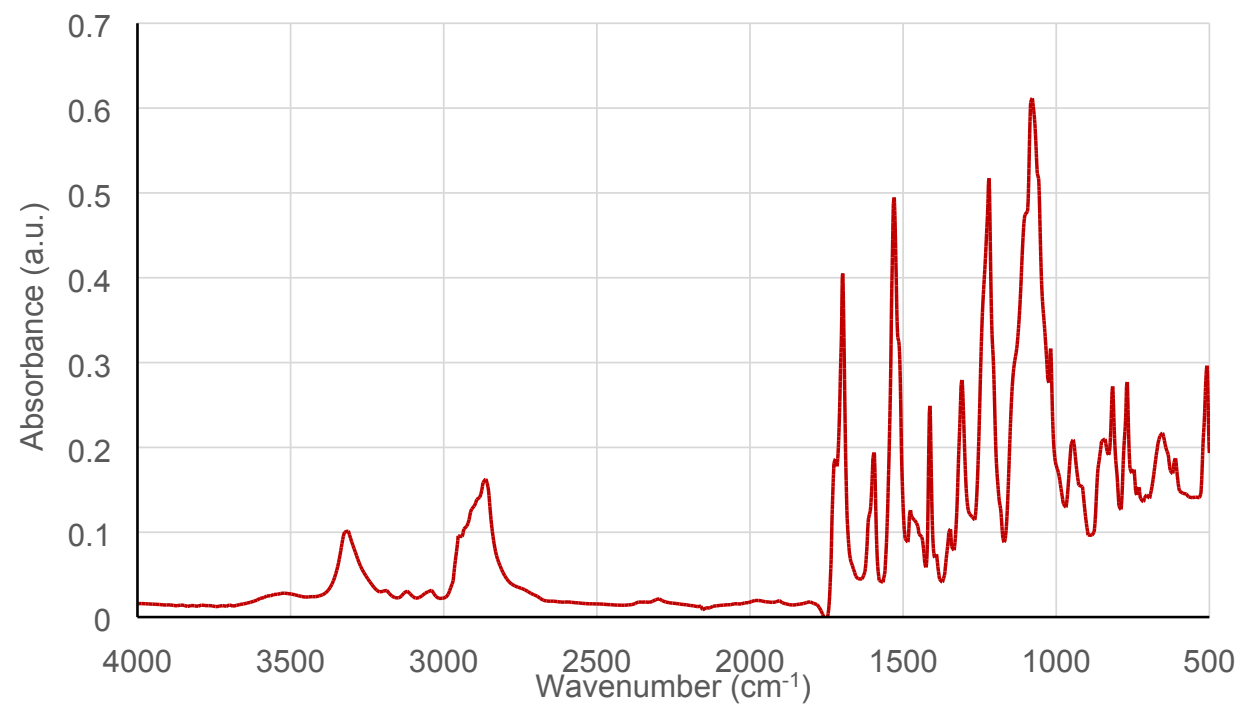

Figure S66: FTIR spectrum of FlowPU4.

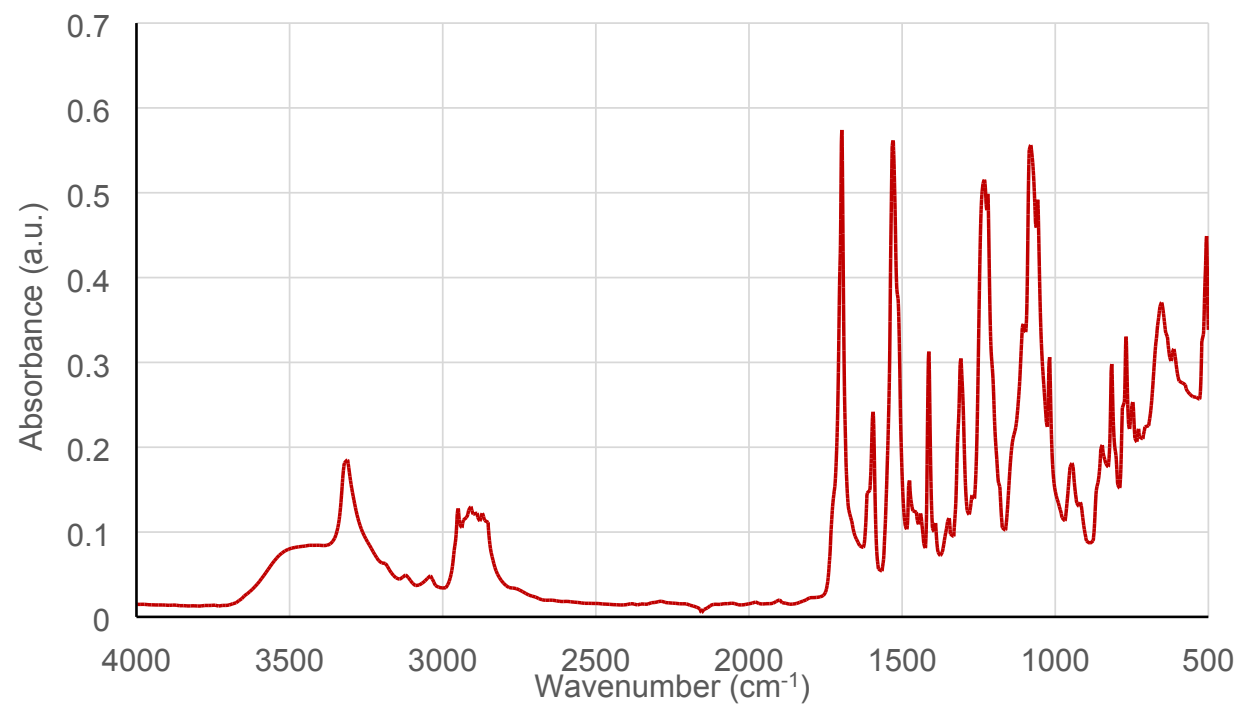

Figure S67: FTIR spectrum of FlowPU5. 


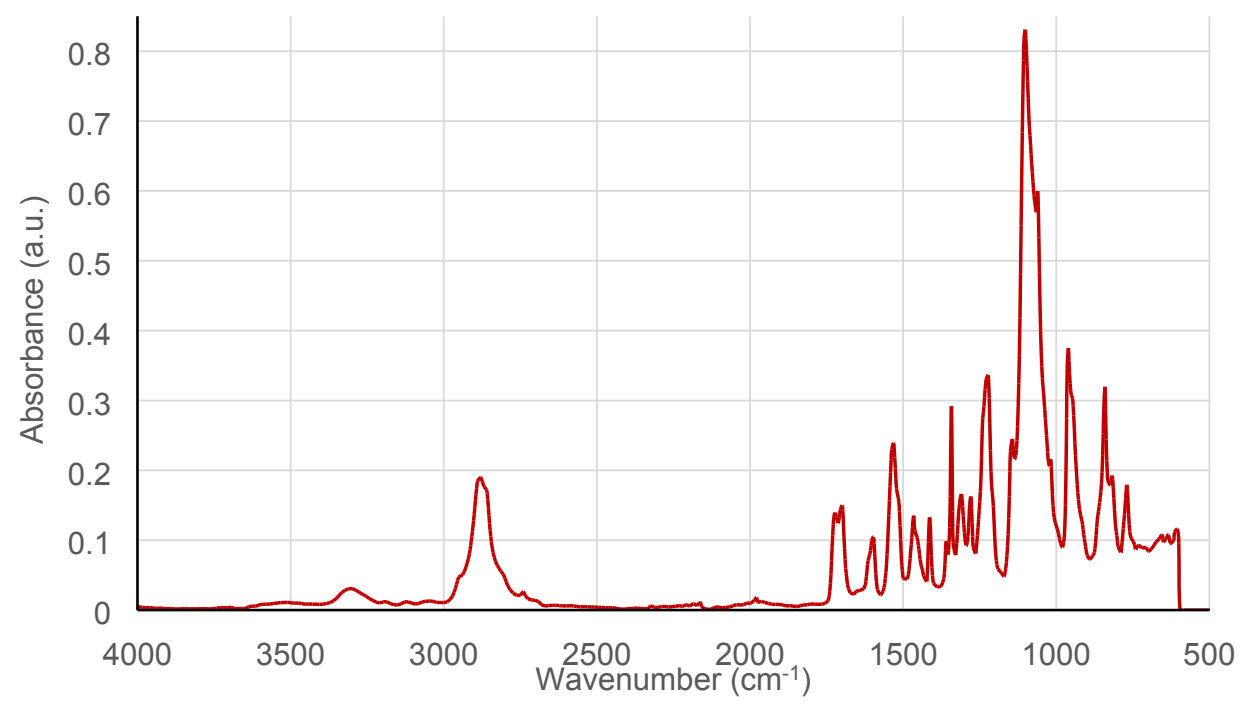

Figure S68: FTIR spectrum of FlowPU6.

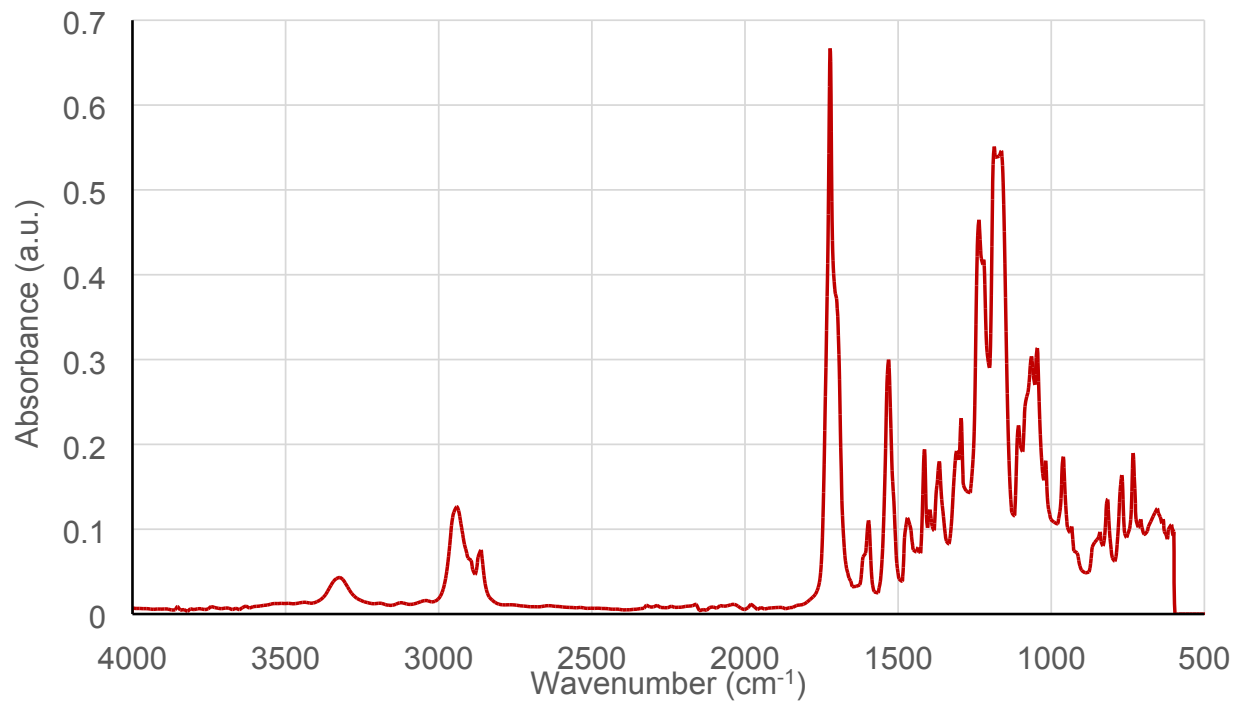

Figure S69: FTIR spectrum of FlowPU7. 


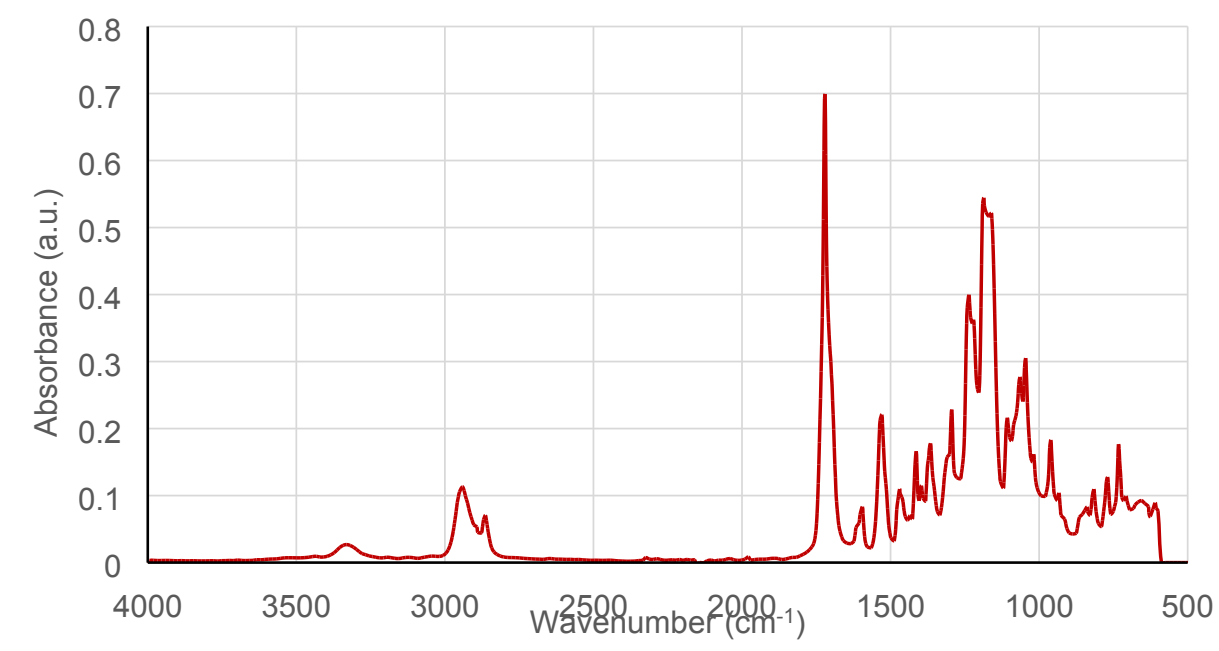

Figure S70: FTIR spectrum of FlowPU8.

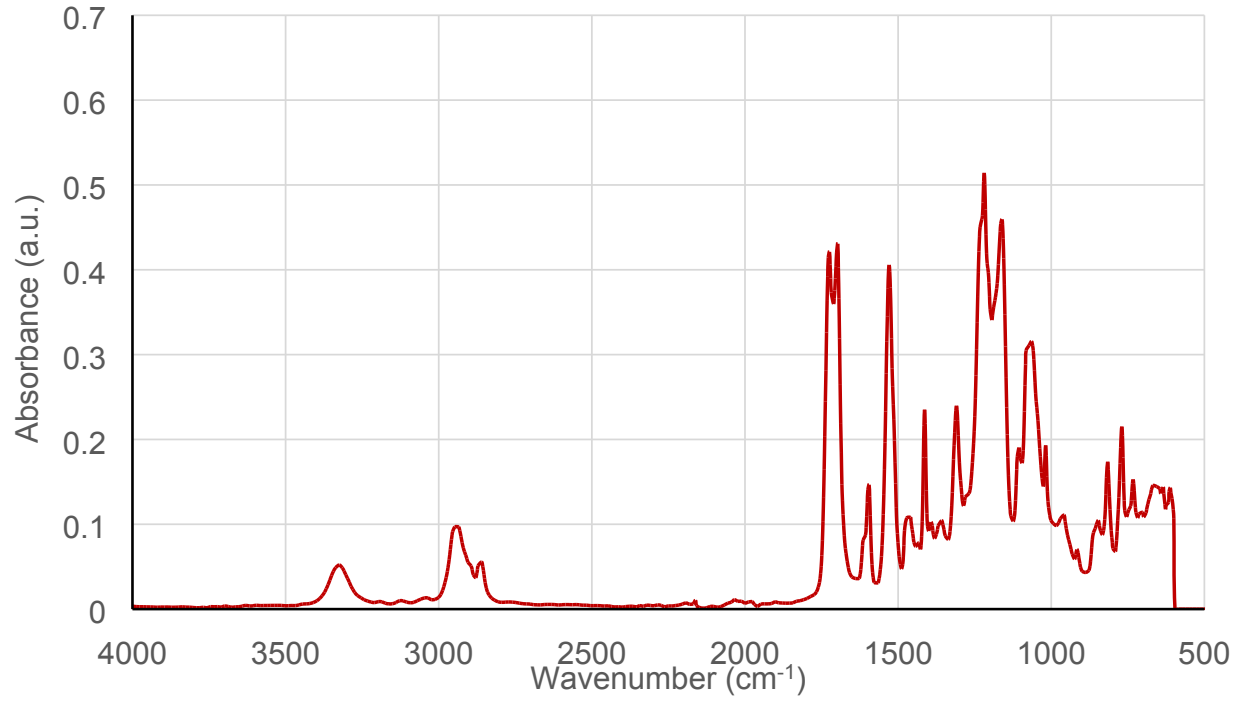

Figure S71: FTIR spectrum of FlowPU9. 


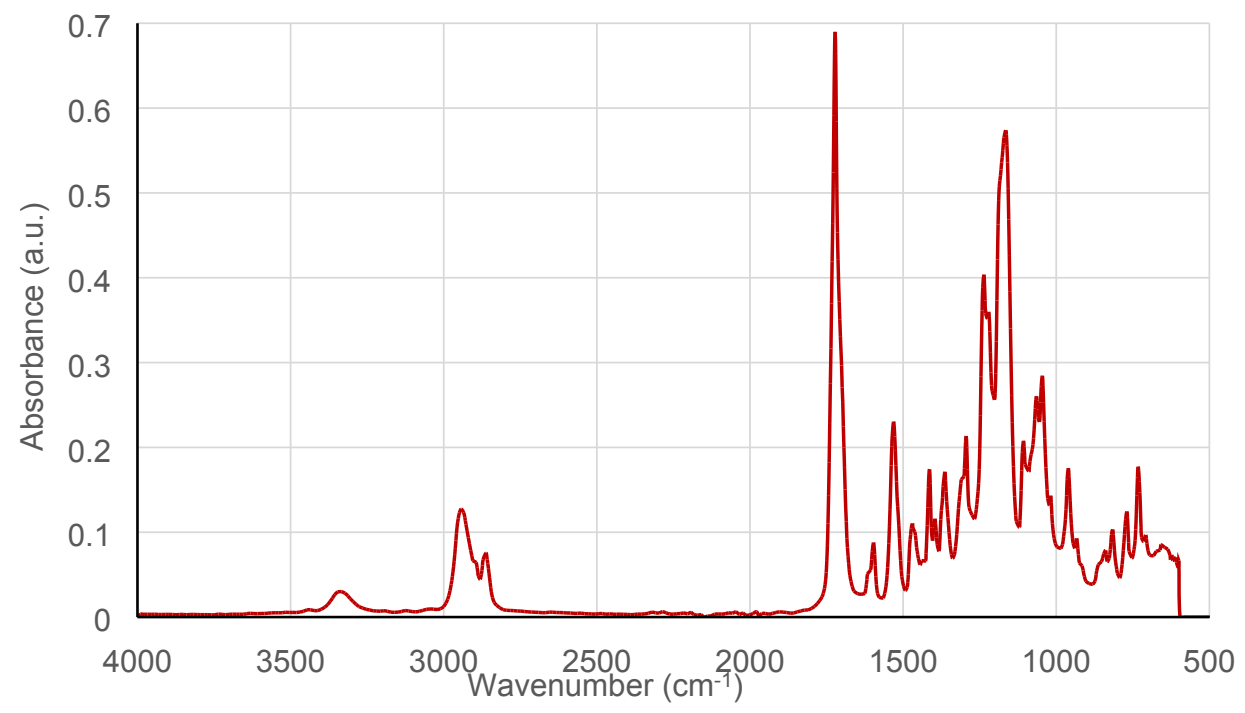

Figure S72: FTIR spectrum of FlowPU10.

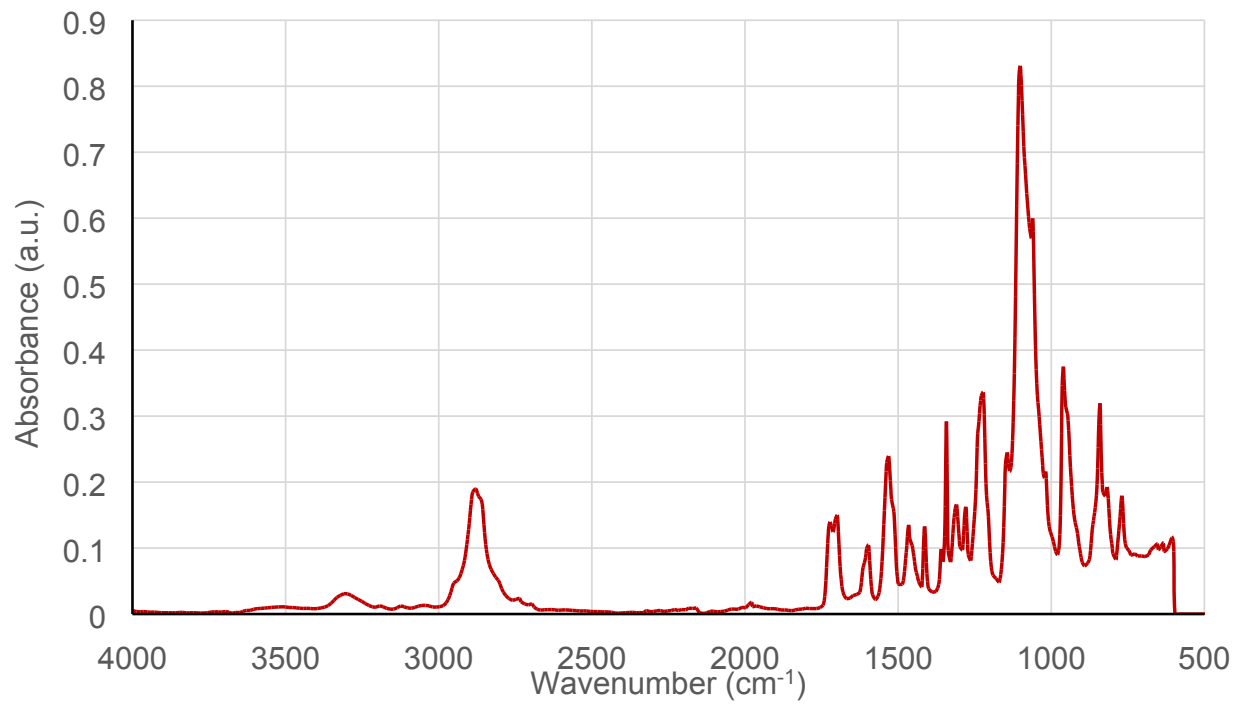

Figure S73: FTIR spectrum of FlowPU11. 


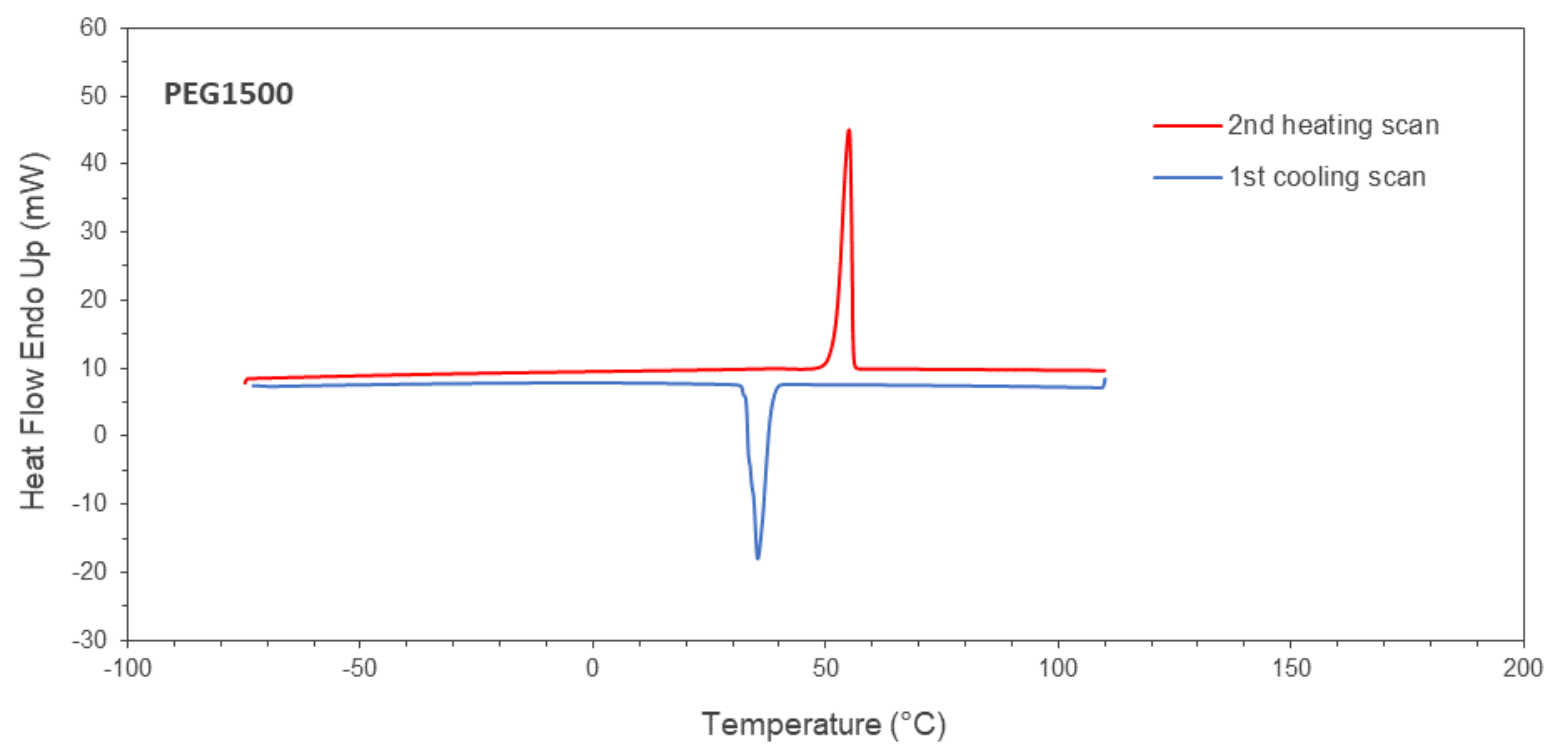

Figure S74: DSC curves of PEG1500 for $2^{\text {nd }}$ heating and $1^{\text {st }}$ cooling scan.

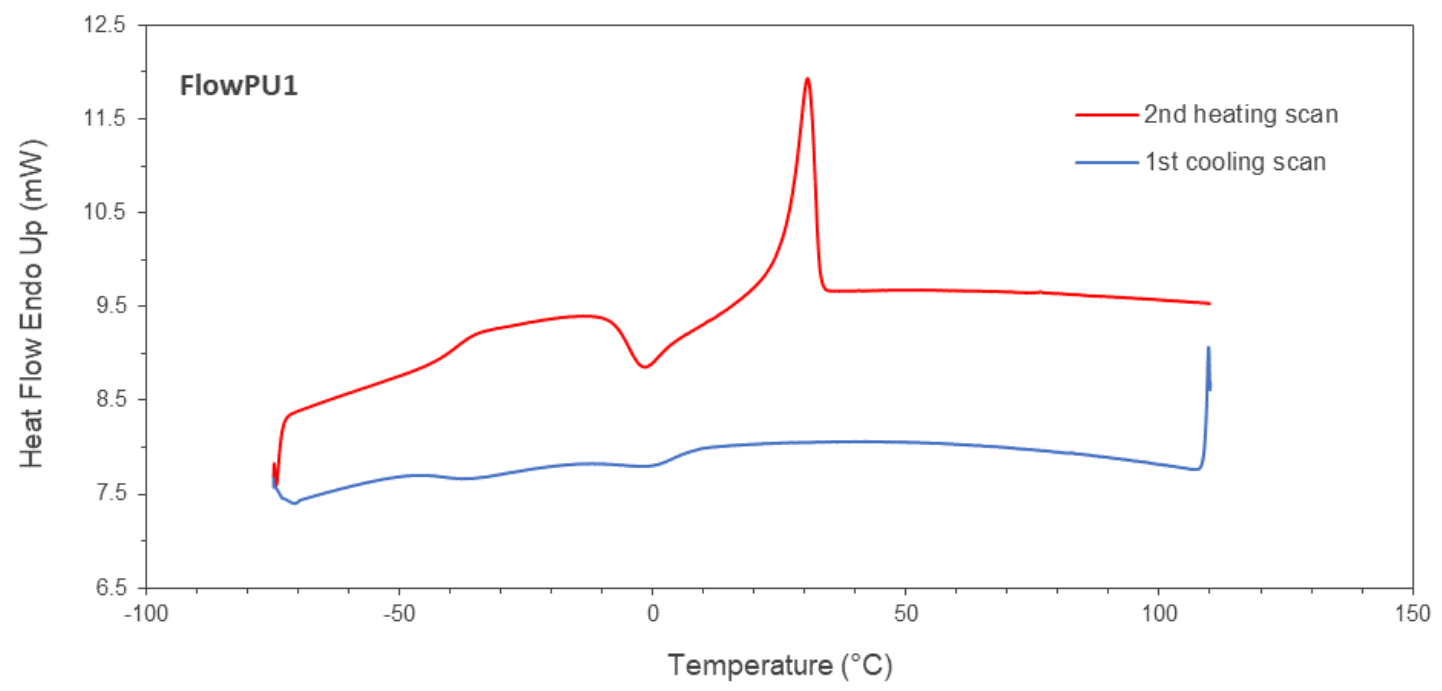

Figure S75: DSC curves of FlowPU1 for $2^{\text {nd }}$ heating and $1^{\text {st }}$ cooling scan. 


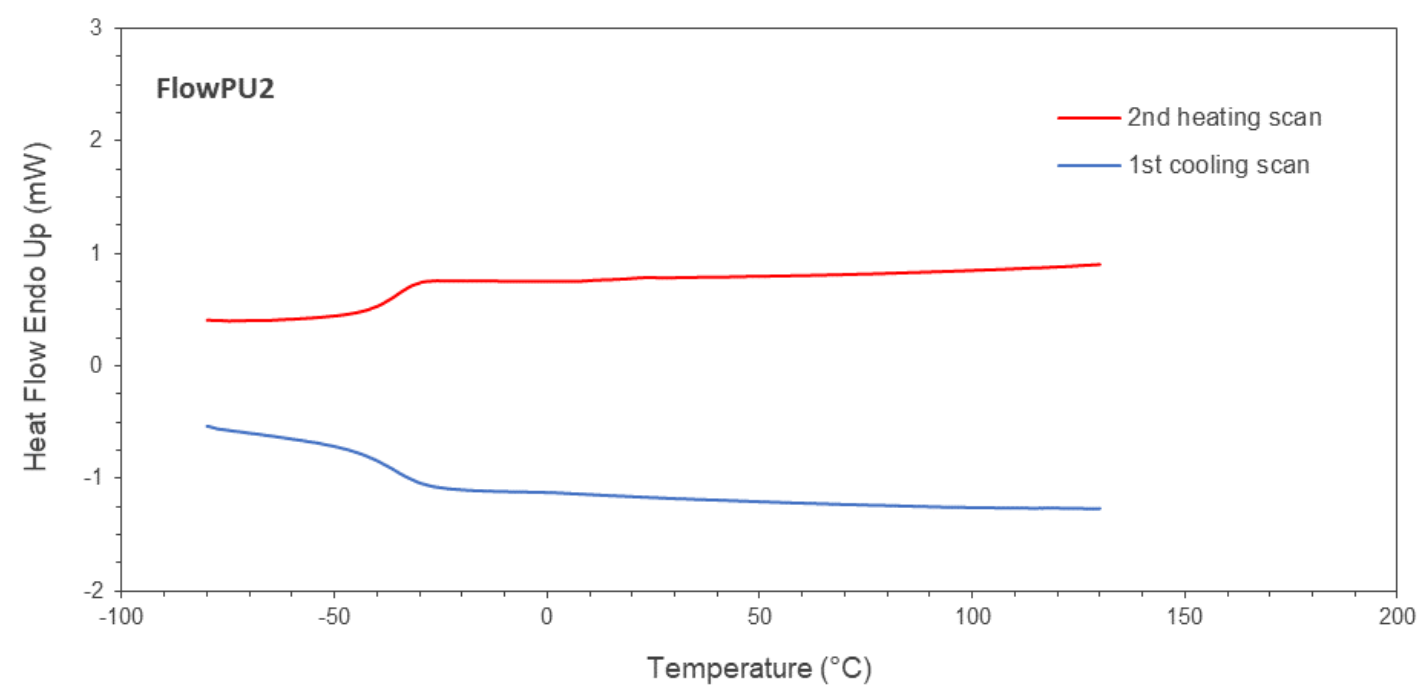

Figure S76: DSC curves of FlowPU2 for $2^{\text {nd }}$ heating and $1^{\text {st }}$ cooling scan.

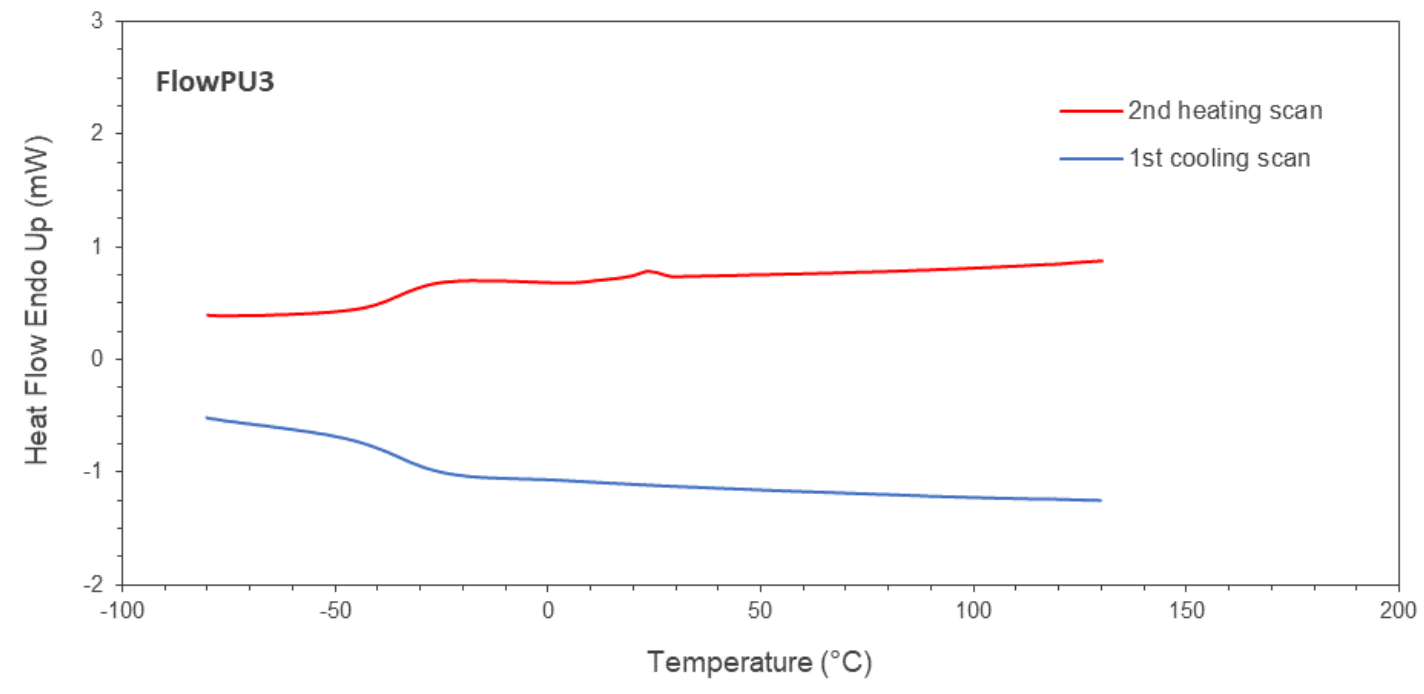

Figure S77: DSC curves of FlowPU3 for $2^{\text {nd }}$ heating and $1^{\text {st }}$ cooling scan. 


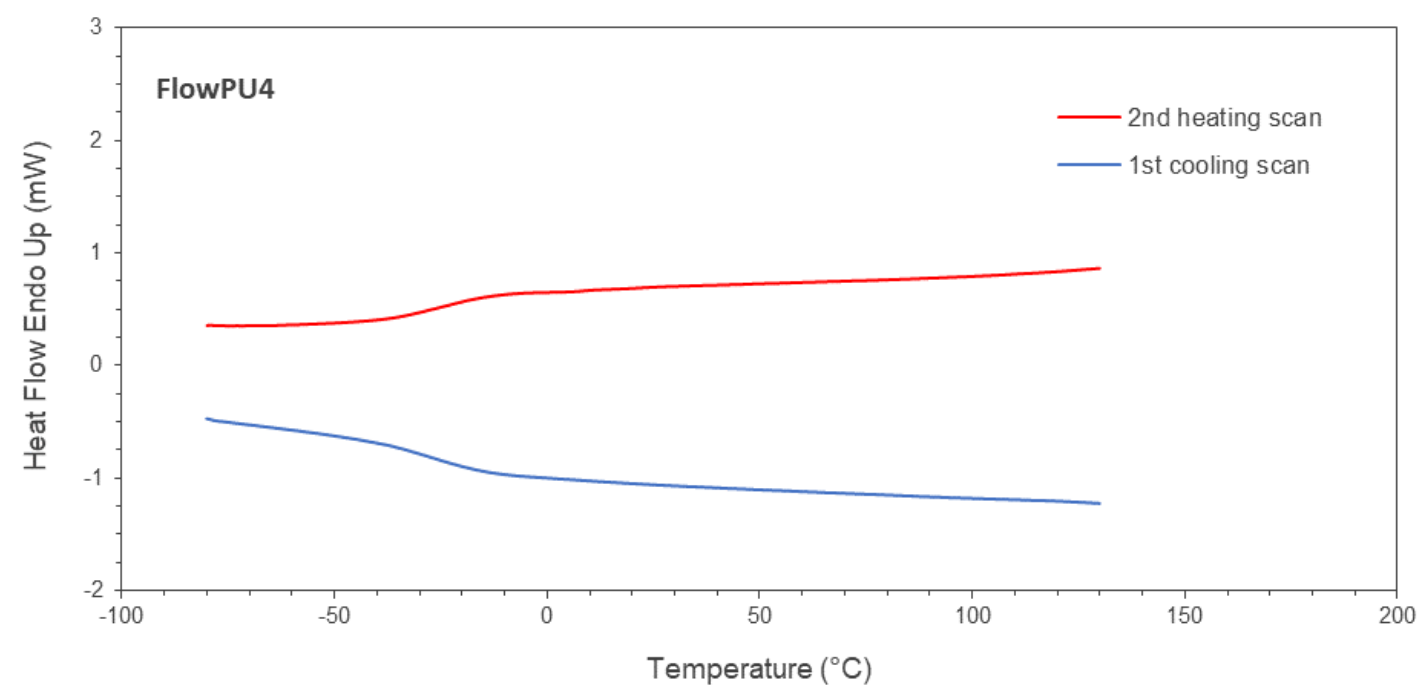

Figure S78: DSC curves of FlowPU4 for $2^{\text {nd }}$ heating and $1^{\text {st }}$ cooling scan.

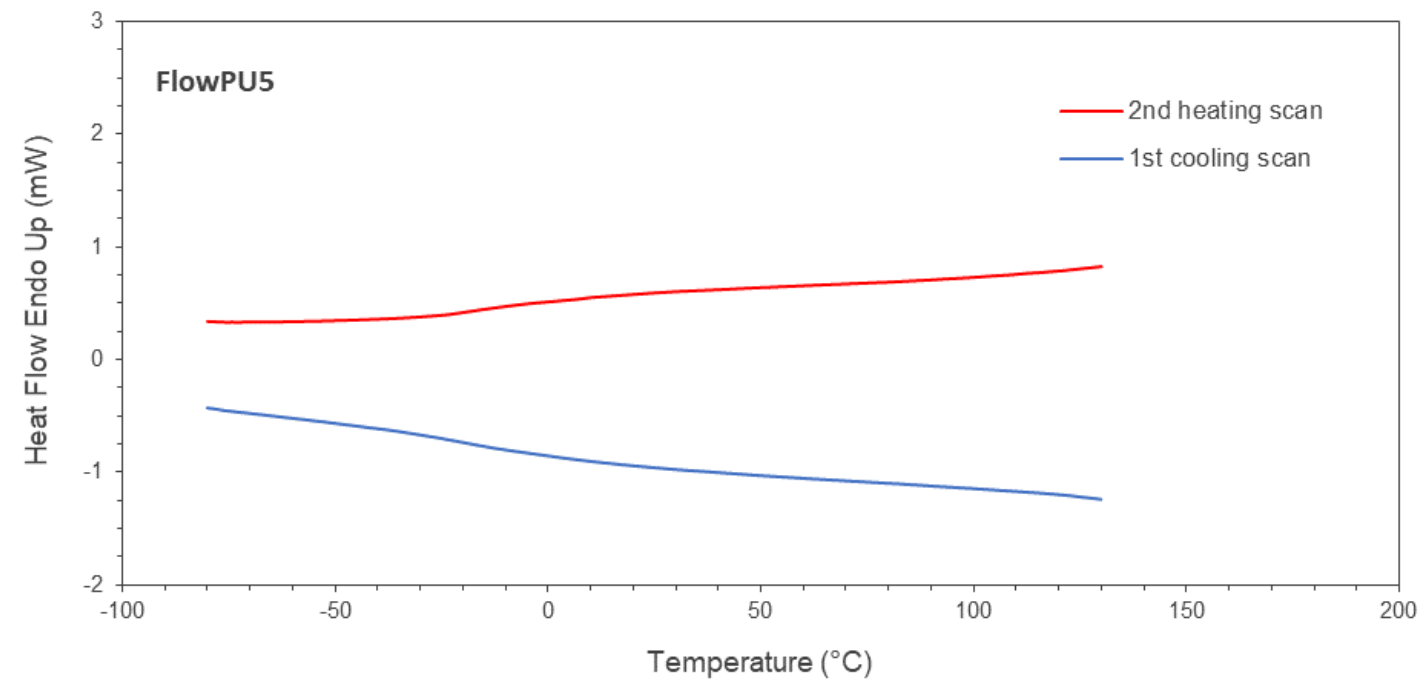

Figure S79: DSC curves of FlowPU5 for $2^{\text {nd }}$ heating and $1^{\text {st }}$ cooling scan. 


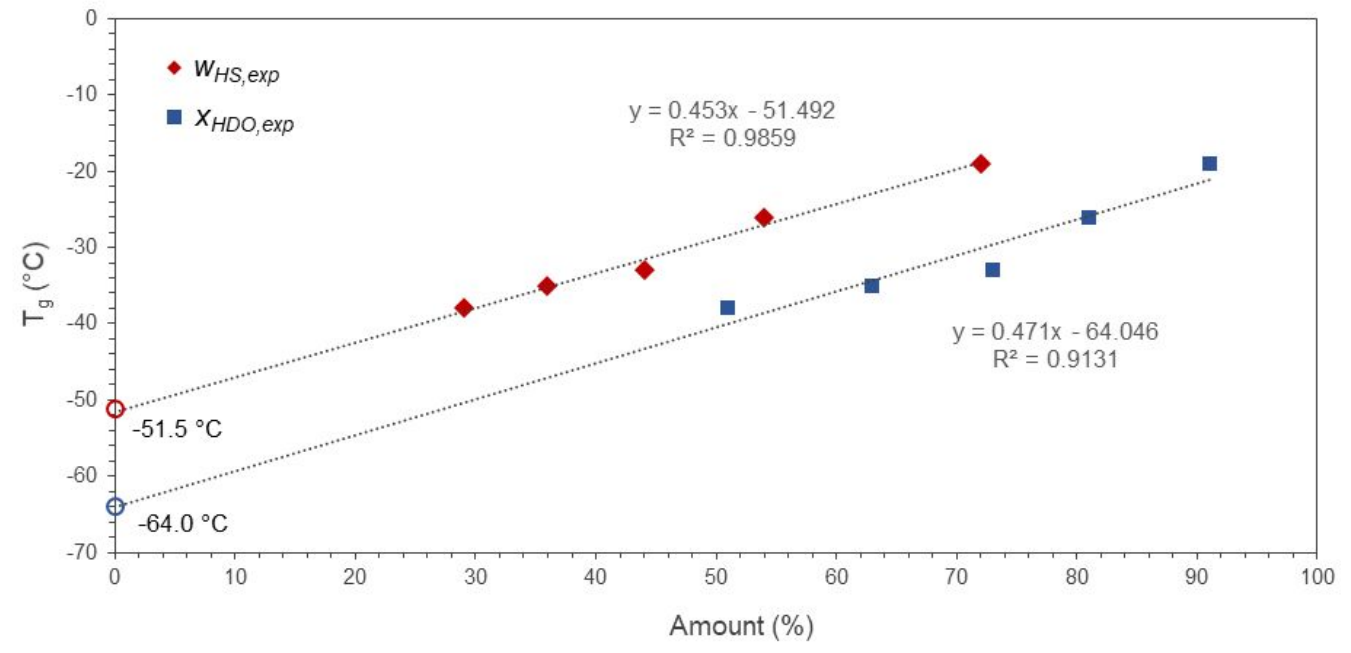

Figure S80: Determined linear relationship for FlowPU1-FlowPU5 between glass transition temperature and weight fraction of the hard segment or molar fraction of HDO in total diols, respectively.

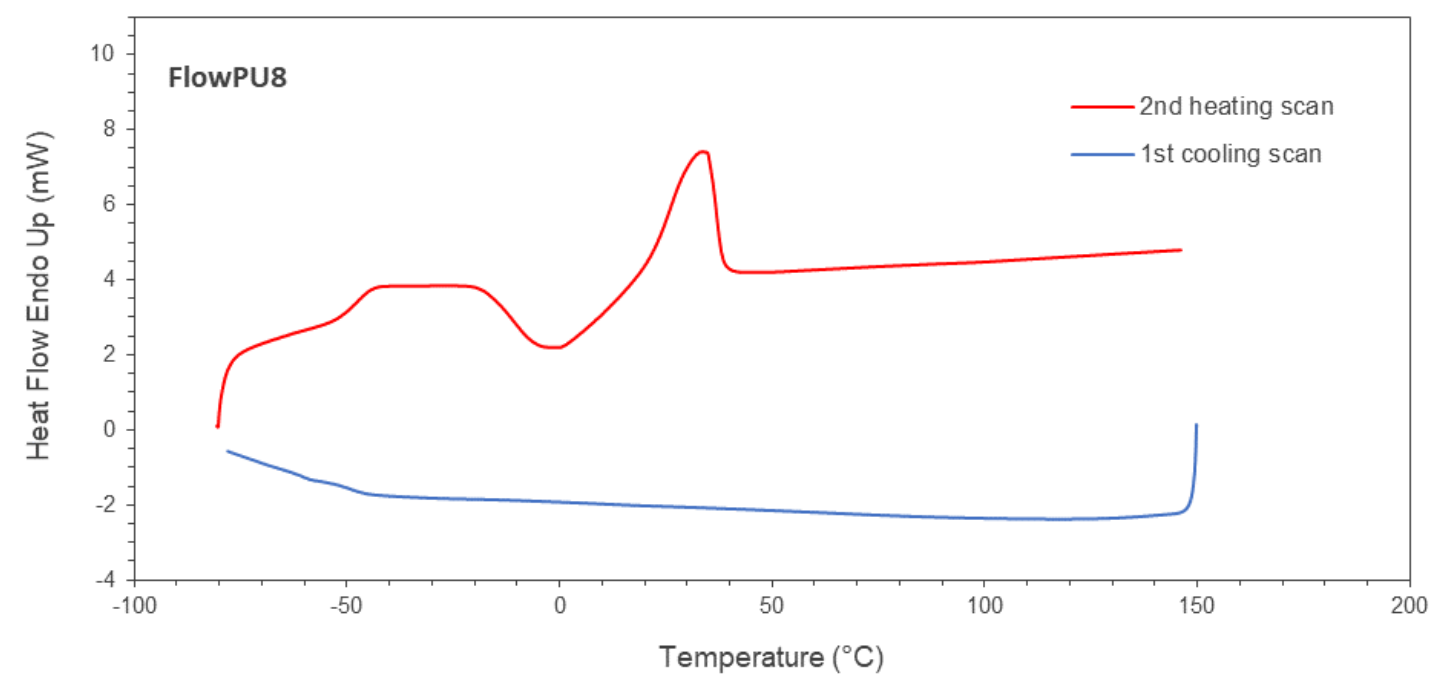

Figure S81: DSC curves of FlowPU8 for $2^{\text {nd }}$ heating and $1^{\text {st }}$ cooling scan. 


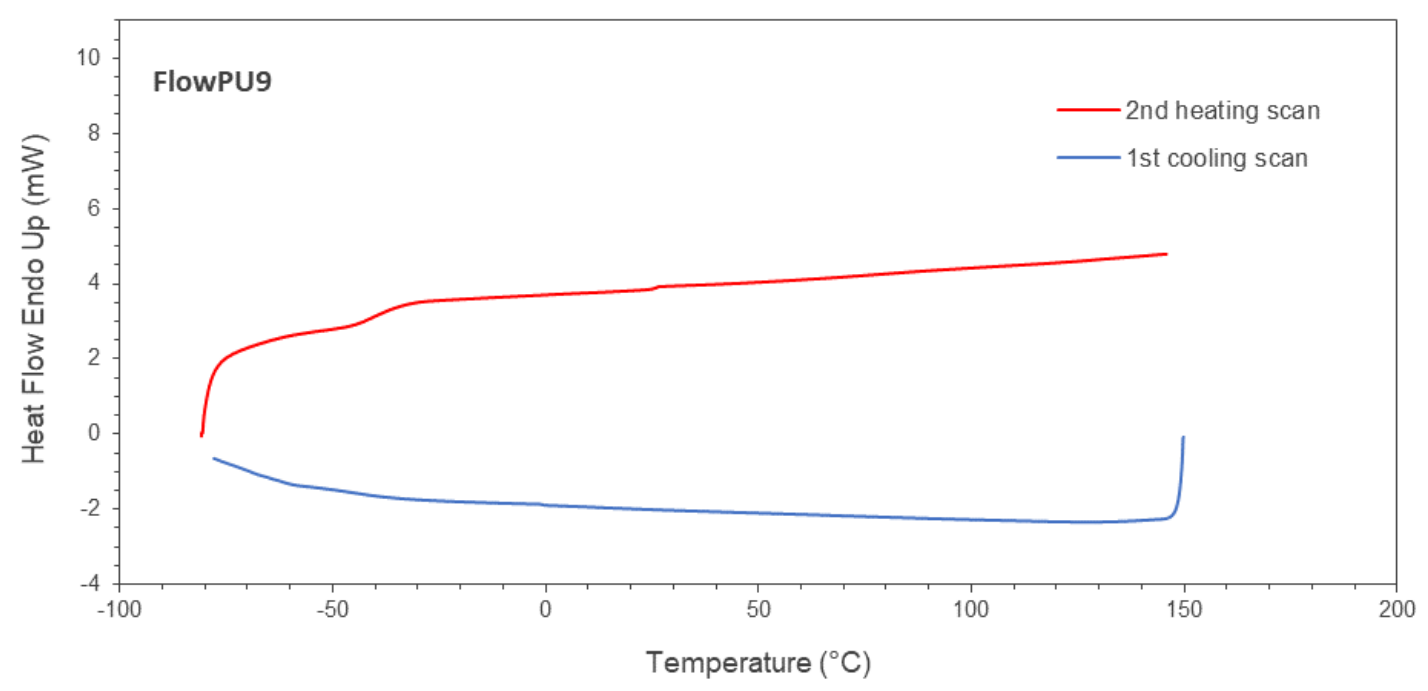

Figure S82: DSC curves of FlowPU9 for $2^{\text {nd }}$ heating and $1^{\text {st }}$ cooling scan.

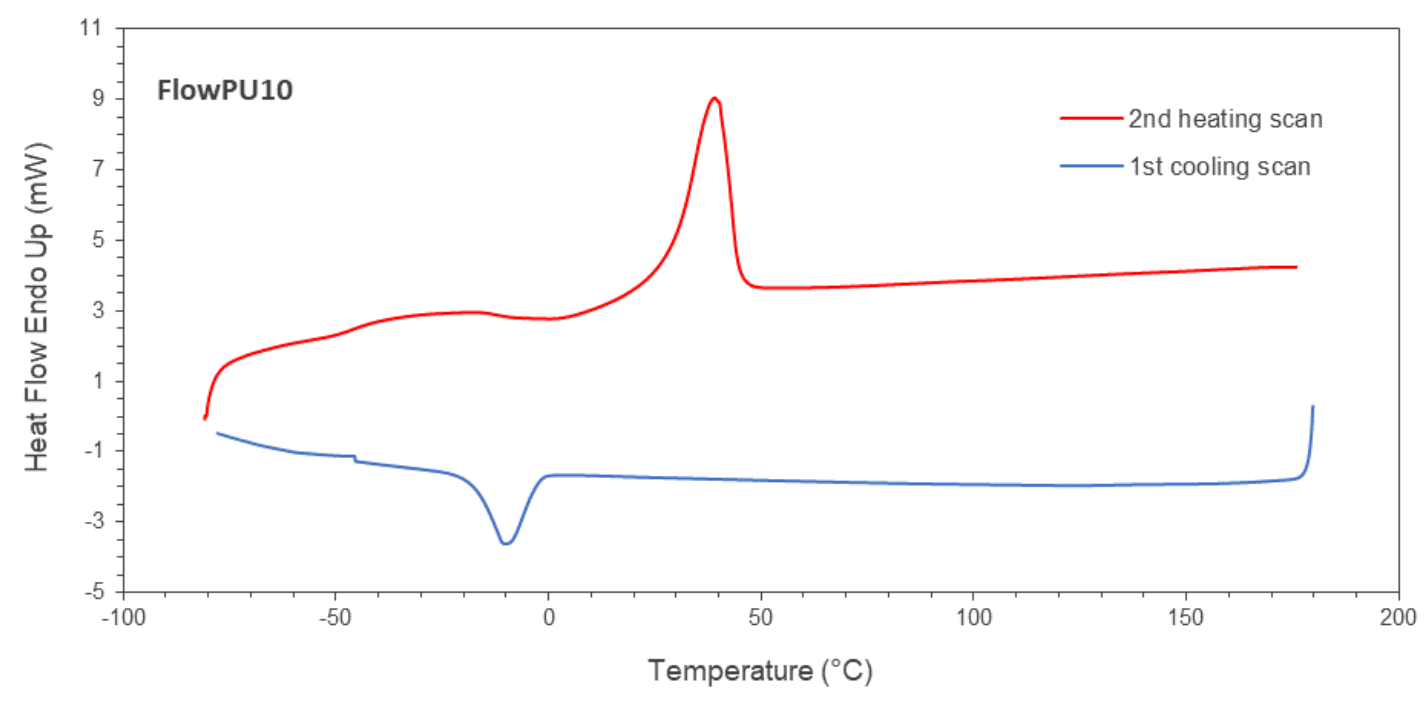

Figure S83: DSC curves of FlowPU10 for $2^{\text {nd }}$ heating and $1^{\text {st }}$ cooling scan. 


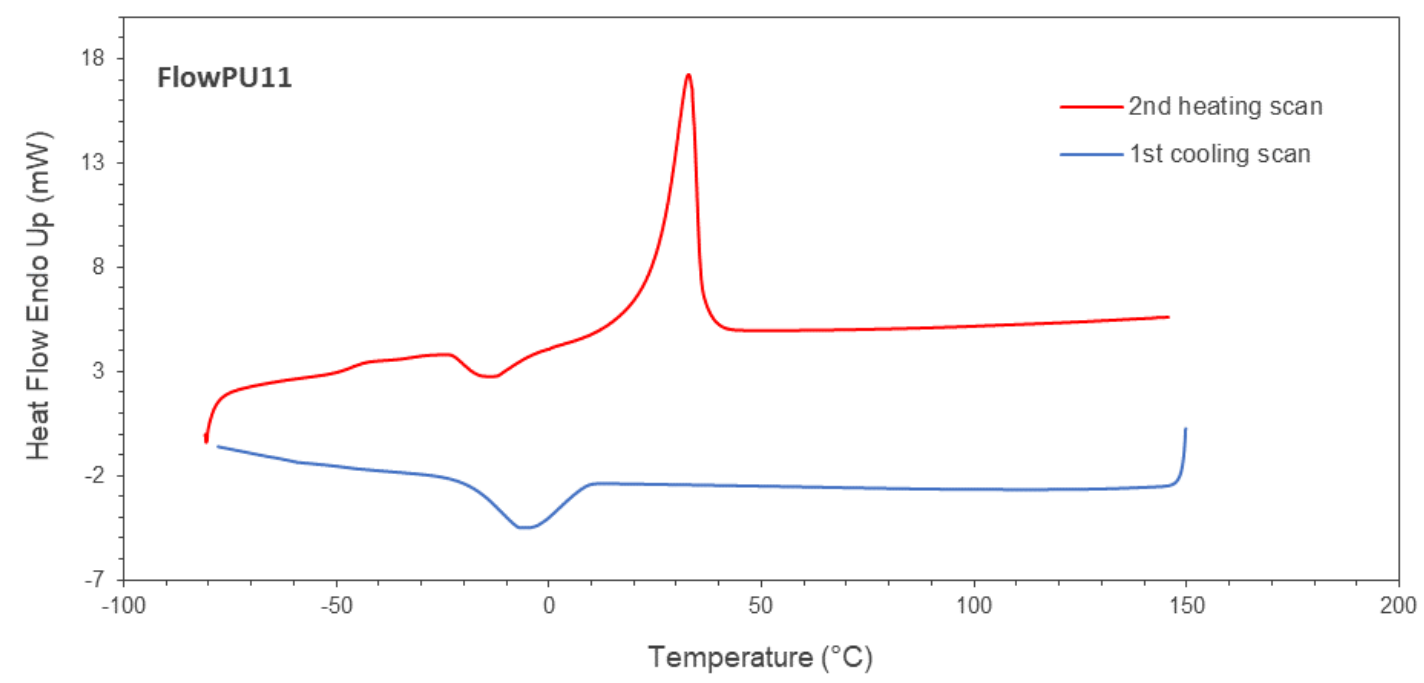

Figure S84: DSC curves of FlowPU11 for $2^{\text {nd }}$ heating and $1^{\text {st }}$ cooling scan.

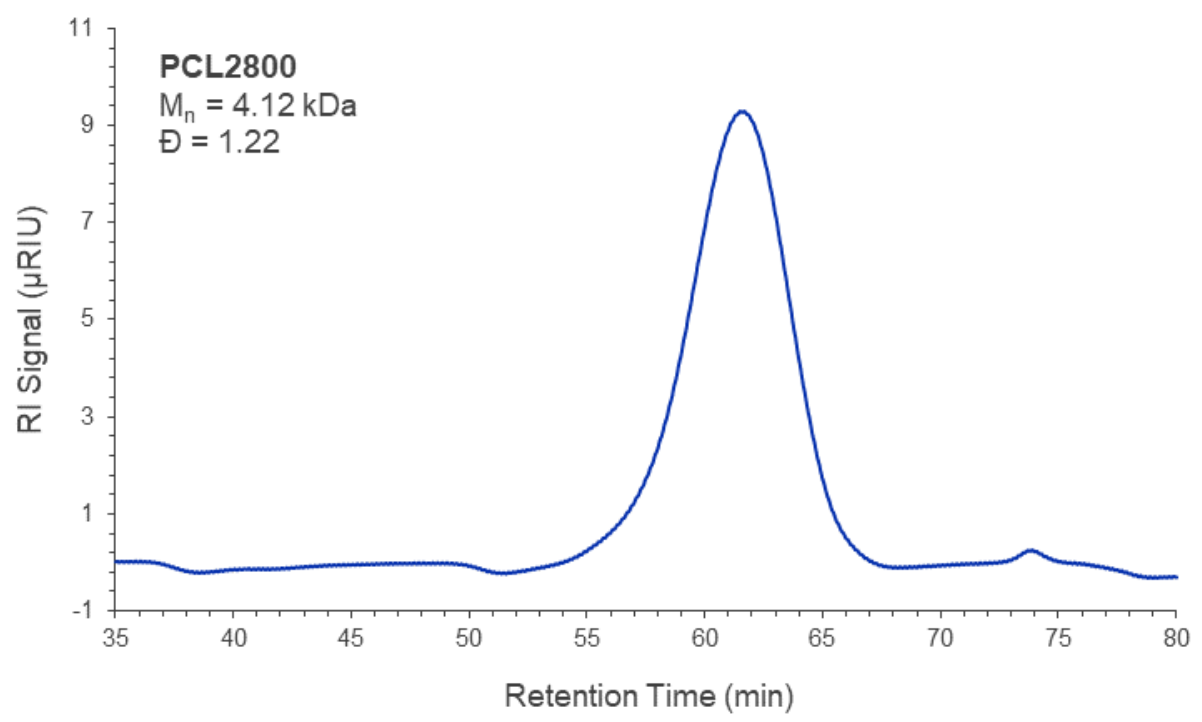

Figure S85: GPC trace of PCL2800. 


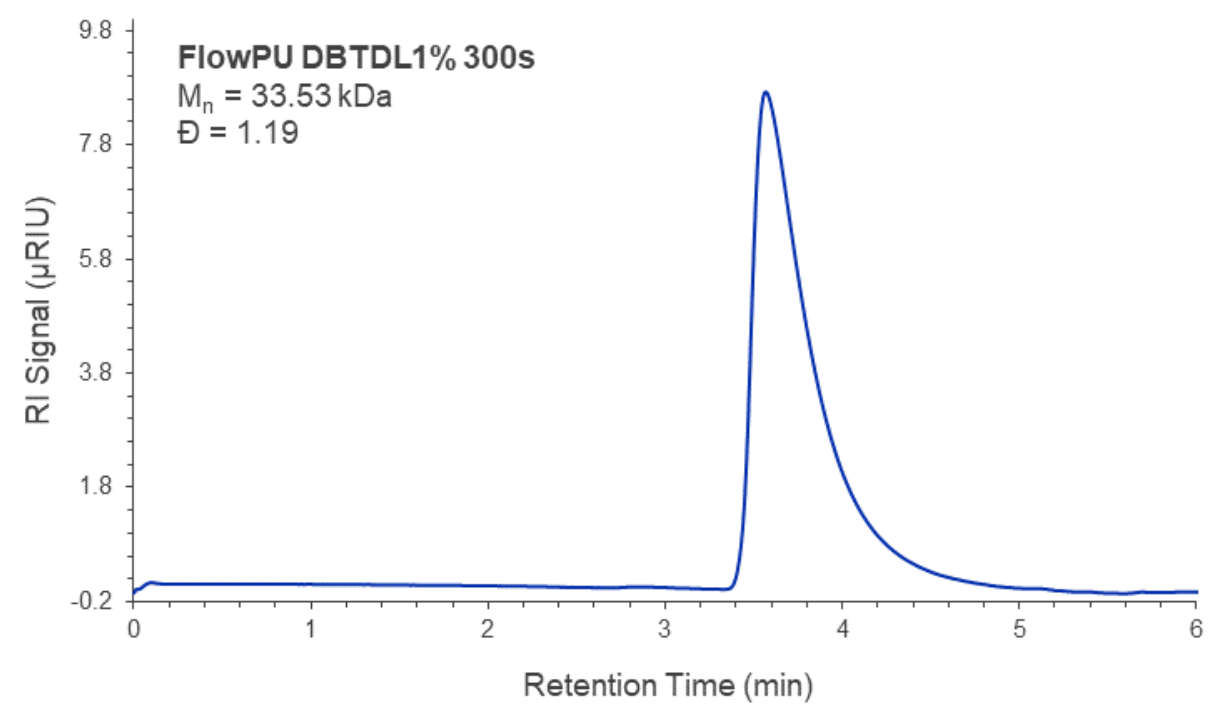

Figure S86: GPC trace of FlowPU DBTDL1\% prepared by using a residence time of $300 \mathrm{~s}$.

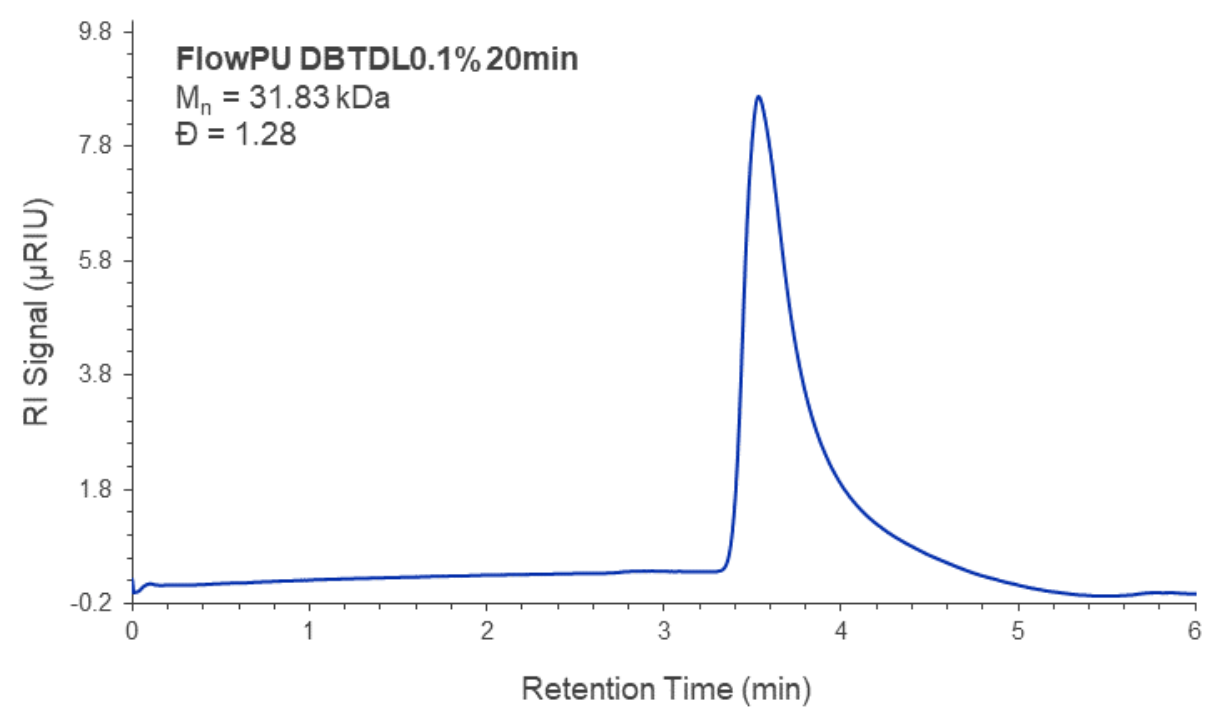

Figure S87: GPC trace of FlowPU DBTDL0.1\% prepared by using a residence time of $20 \mathrm{~min}$. 


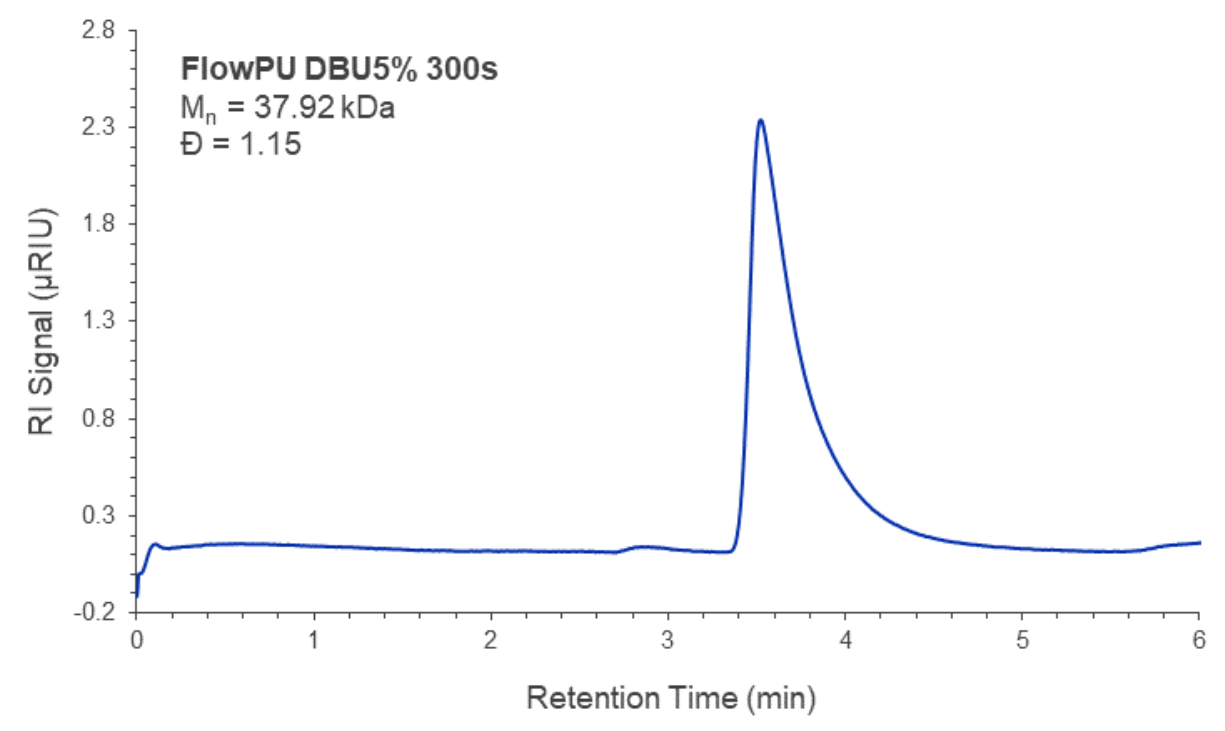

Figure S88: GPC trace of FlowPU DBU5\% prepared by using a residence time of $300 \mathrm{~s}$.

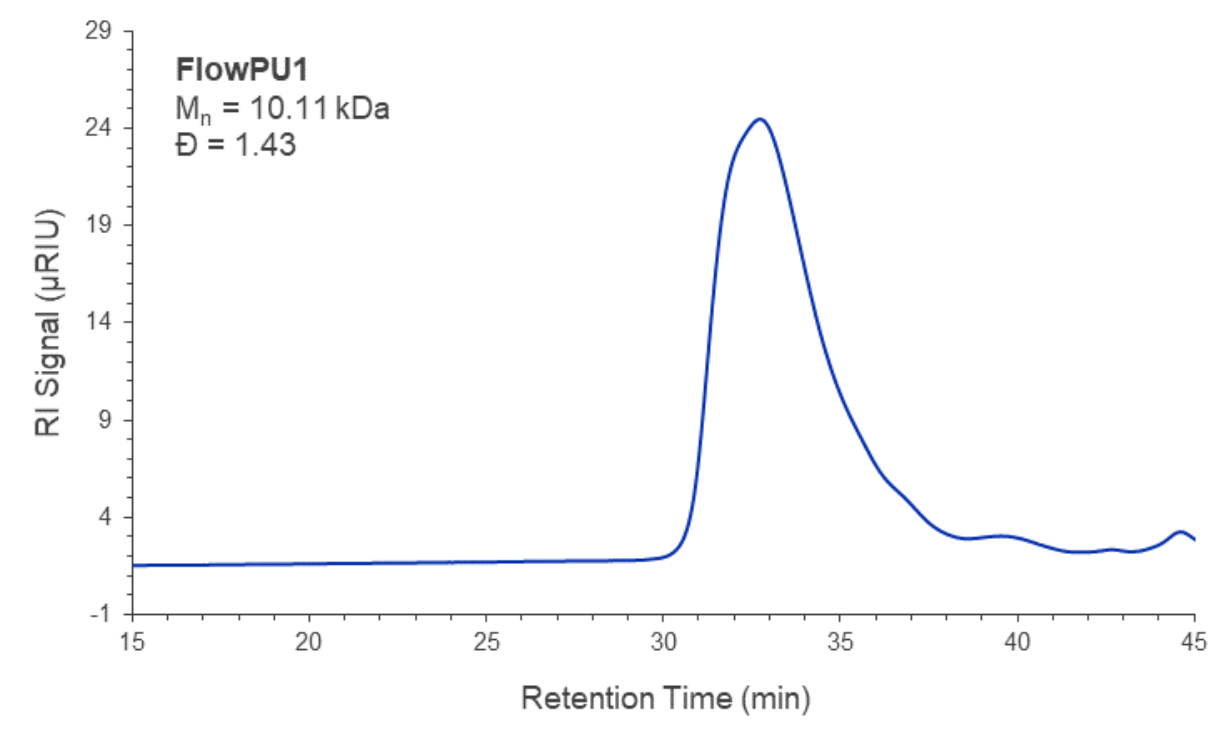

Figure S89: GPC trace of FlowPU1. 


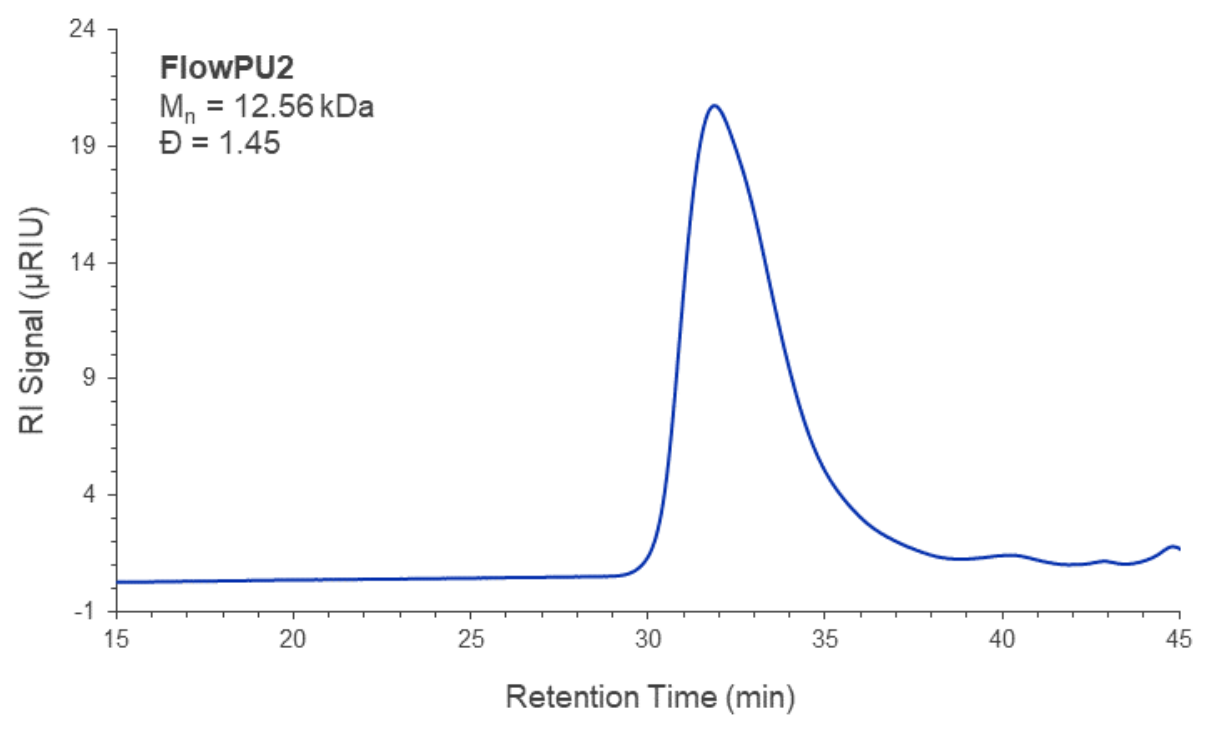

Figure S90: GPC trace of FlowPU2.

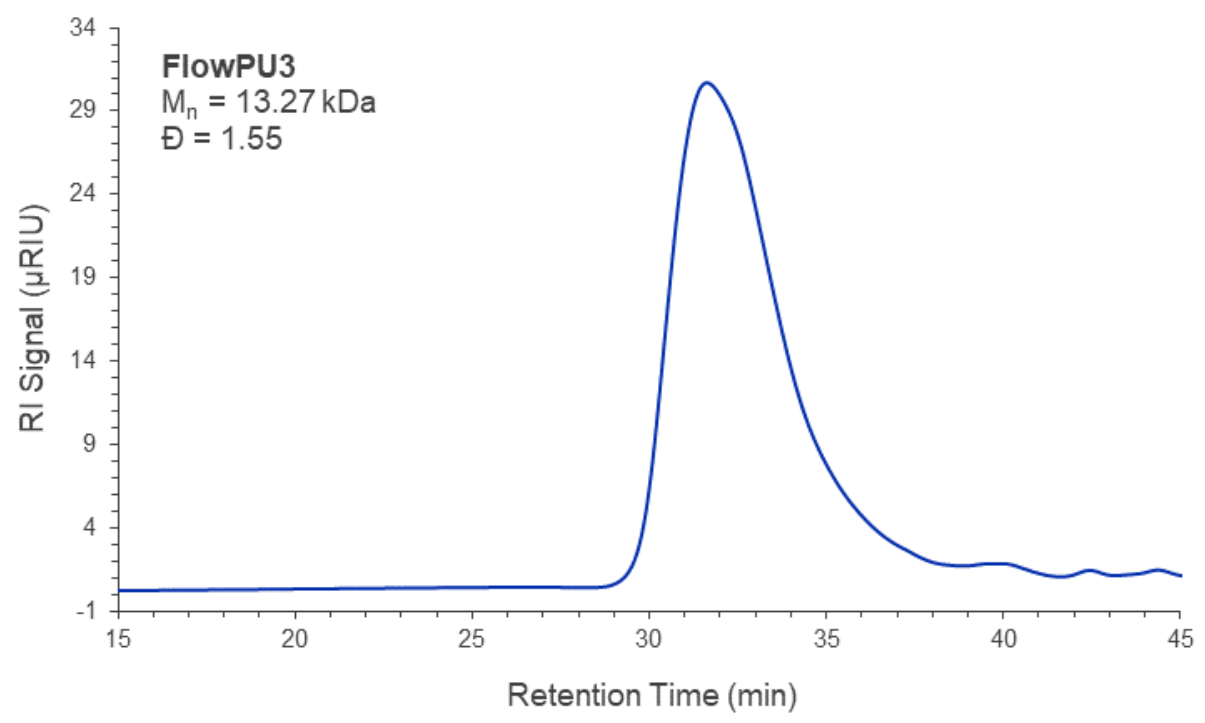

Figure S91: GPC trace of FlowPU3. 


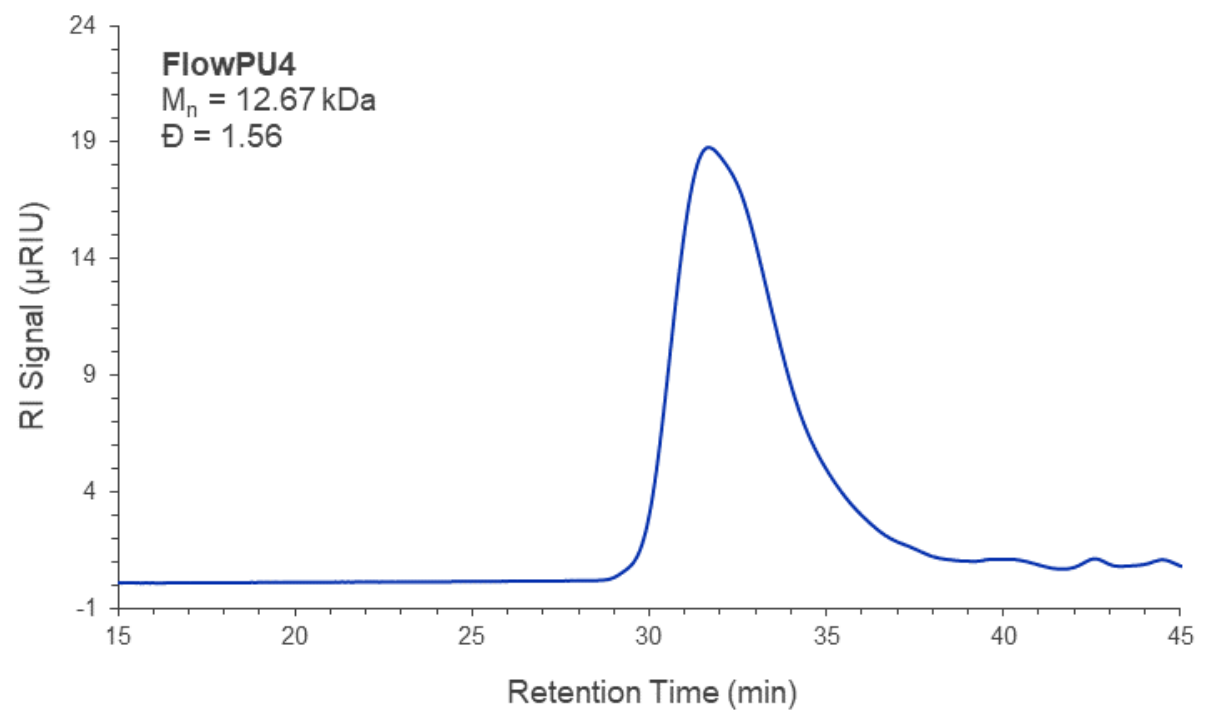

Figure S92: GPC trace of FlowPU4.

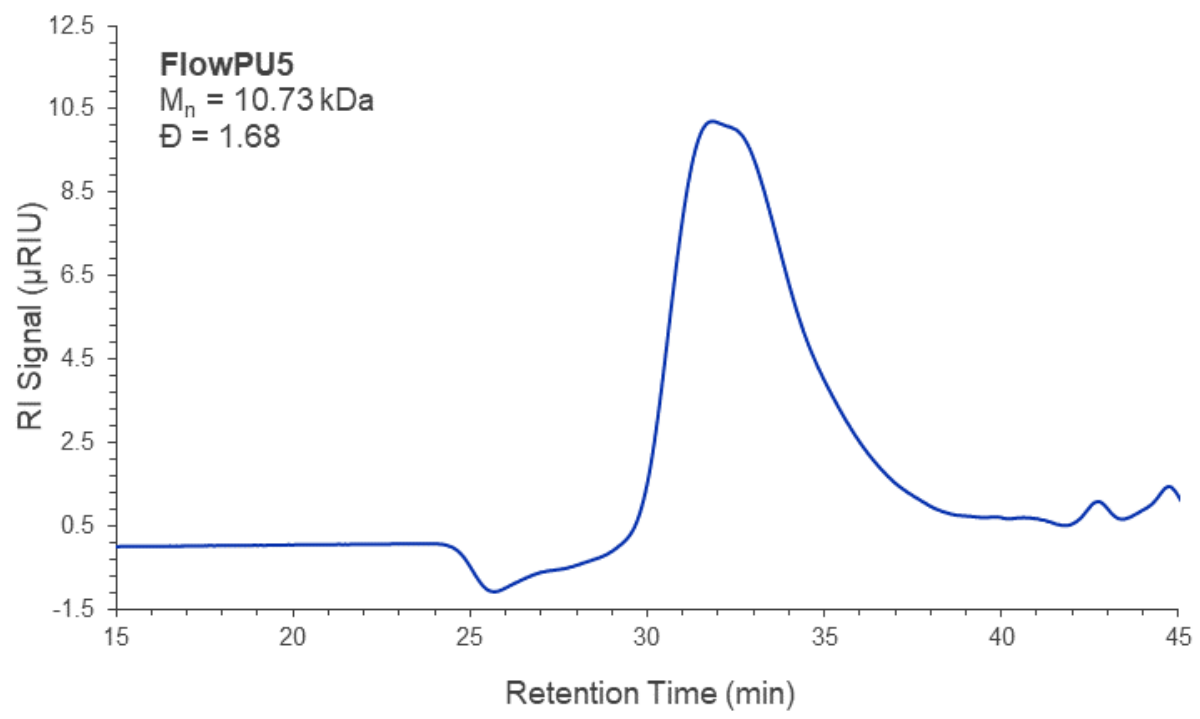

Figure S93: GPC trace of FlowPU5. 


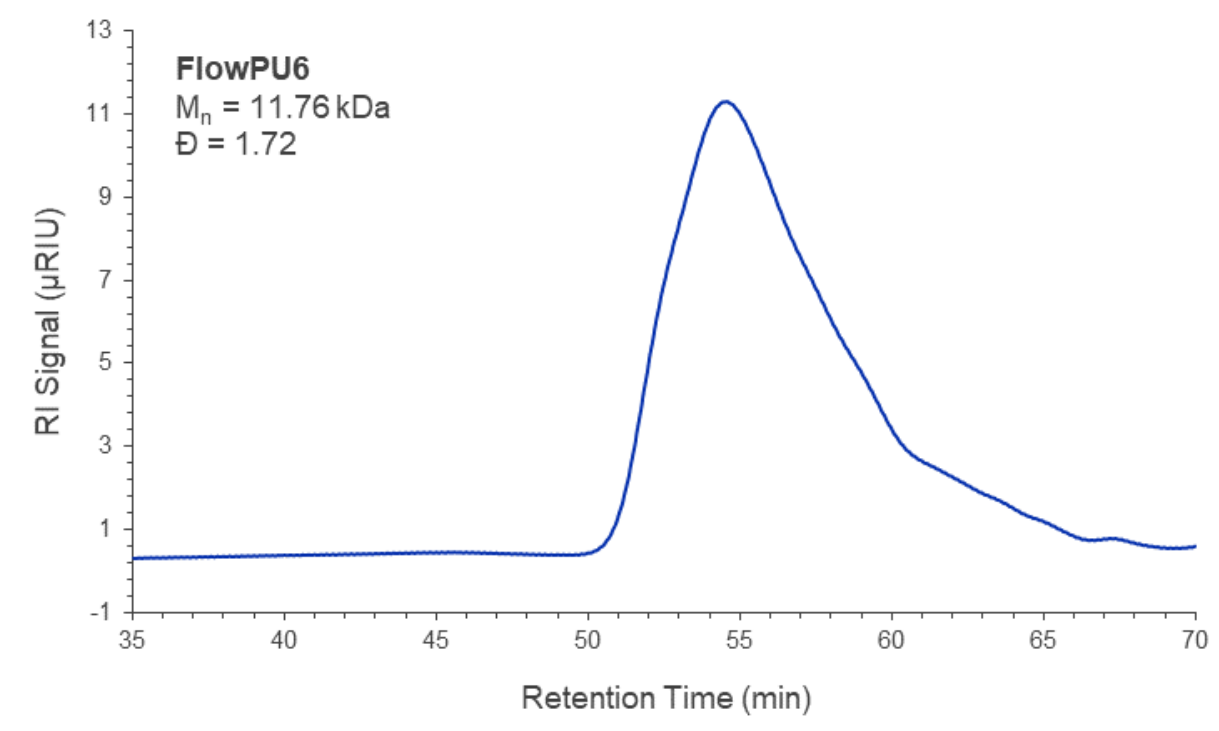

Figure S94: GPC trace of FlowPU6.

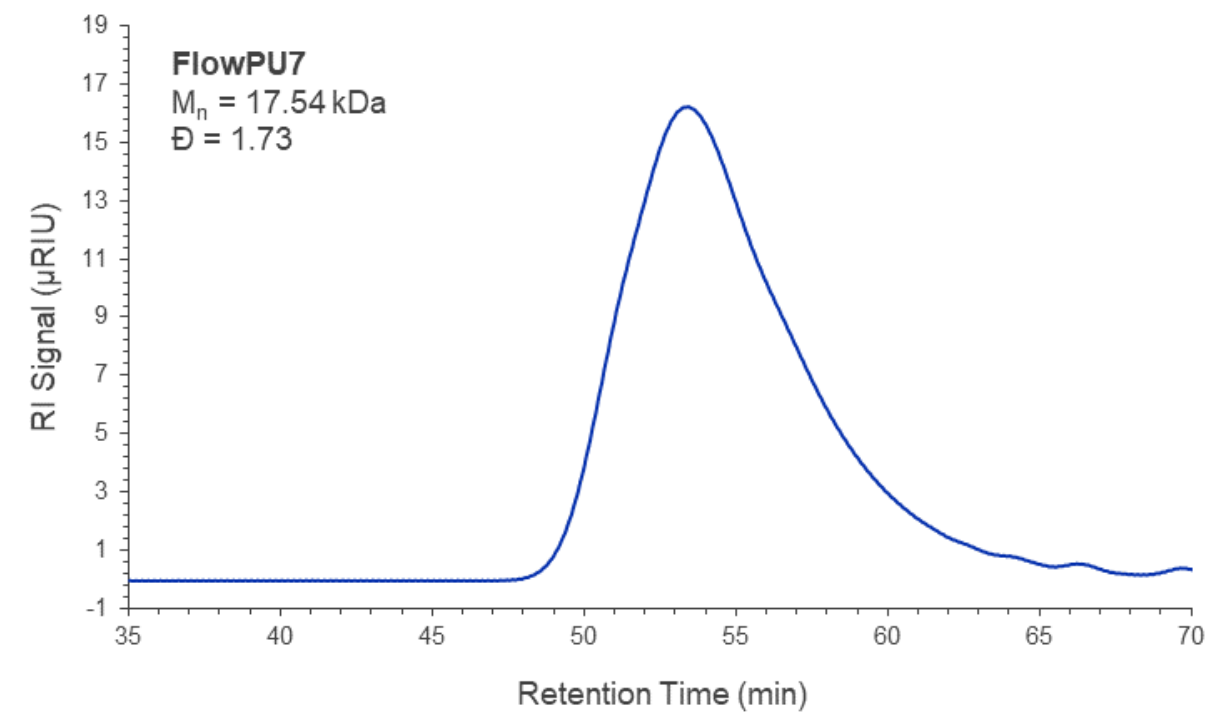

Figure S95: GPC trace of FlowPU7. 


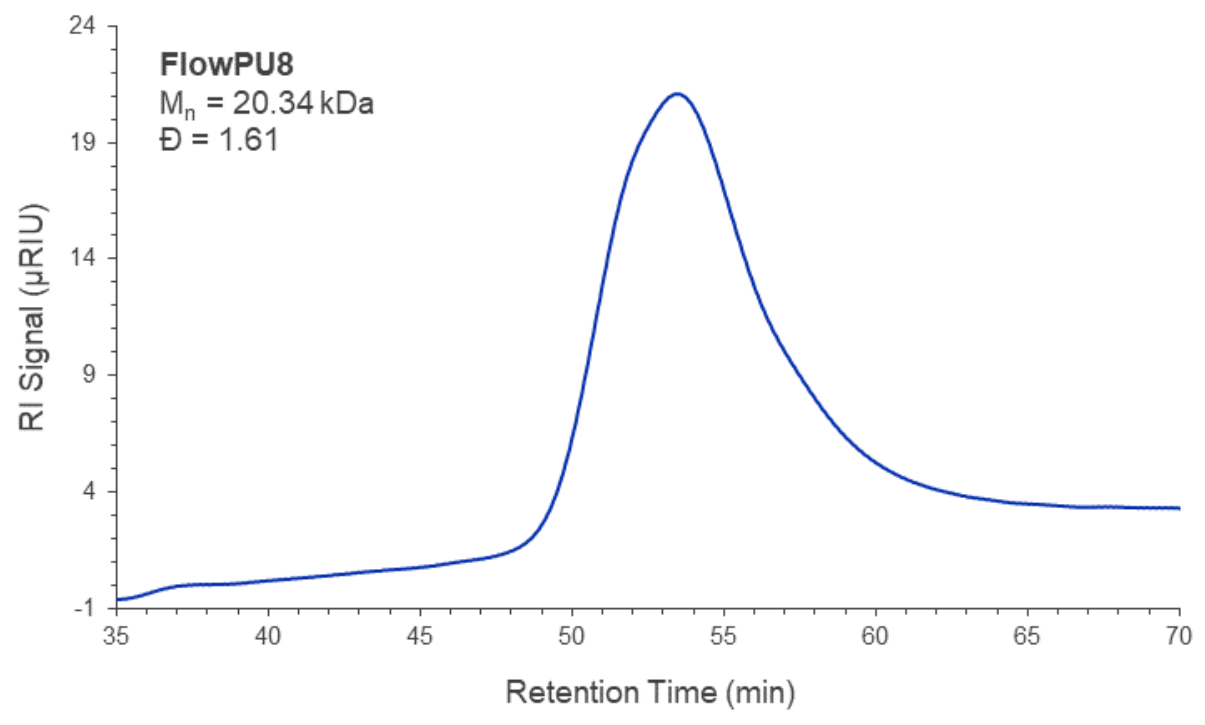

Figure S96: GPC trace of FlowPU8.

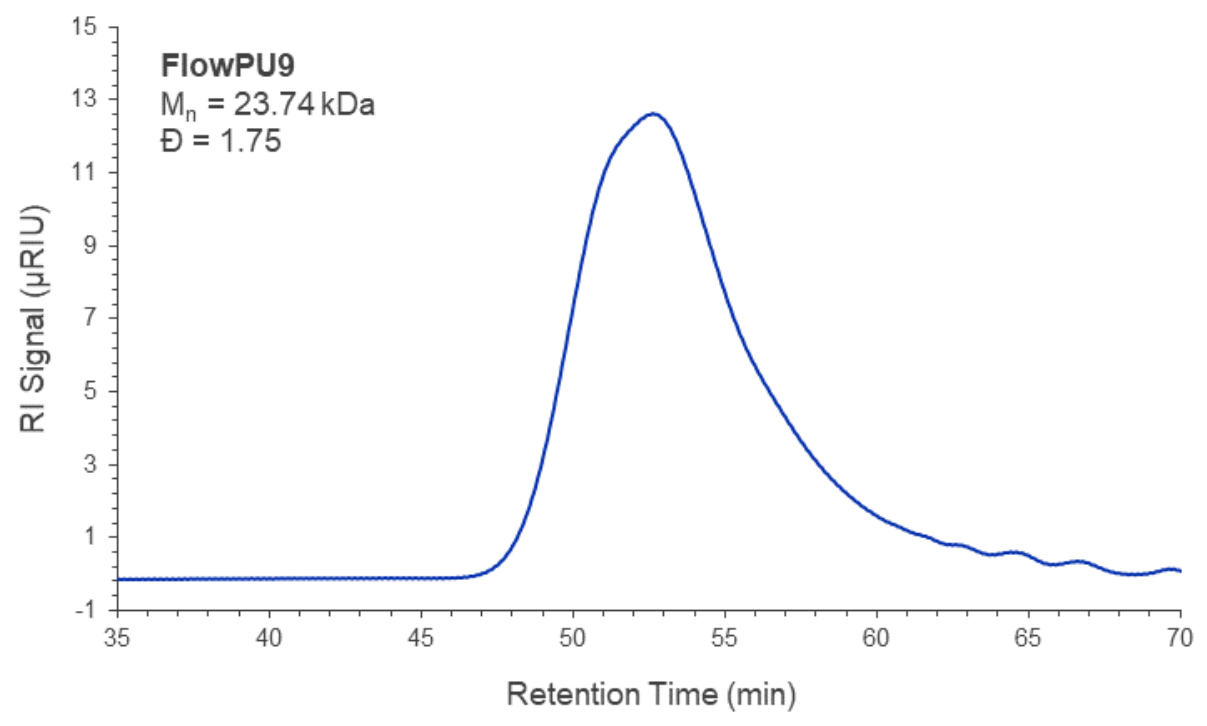

Figure S97: GPC trace of FlowPU9. 


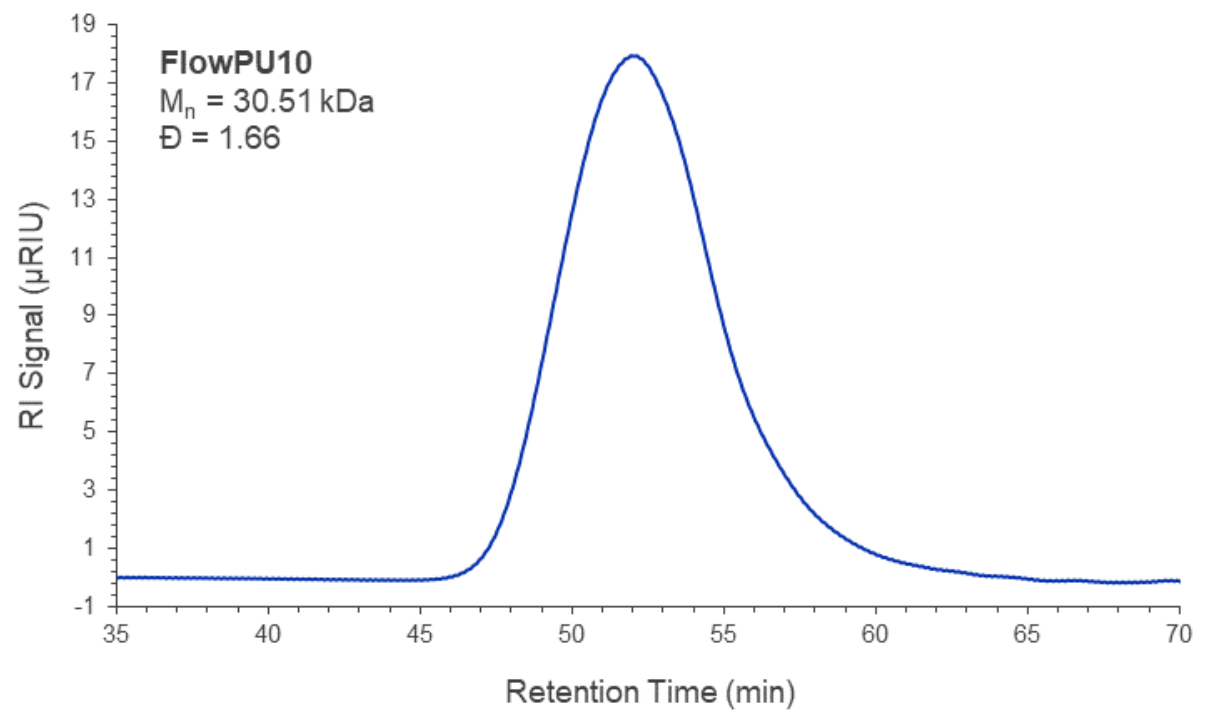

Figure S98: GPC trace of FlowPU10.

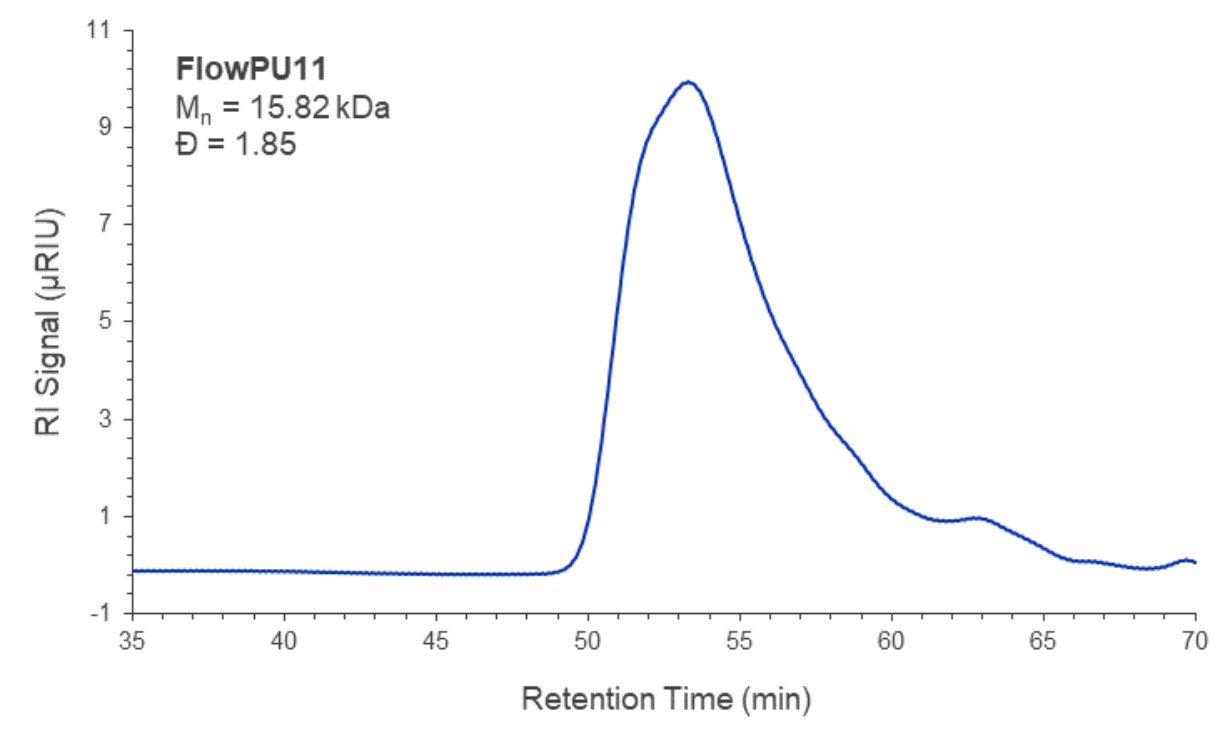

Figure S99: GPC trace of FlowPU11. 


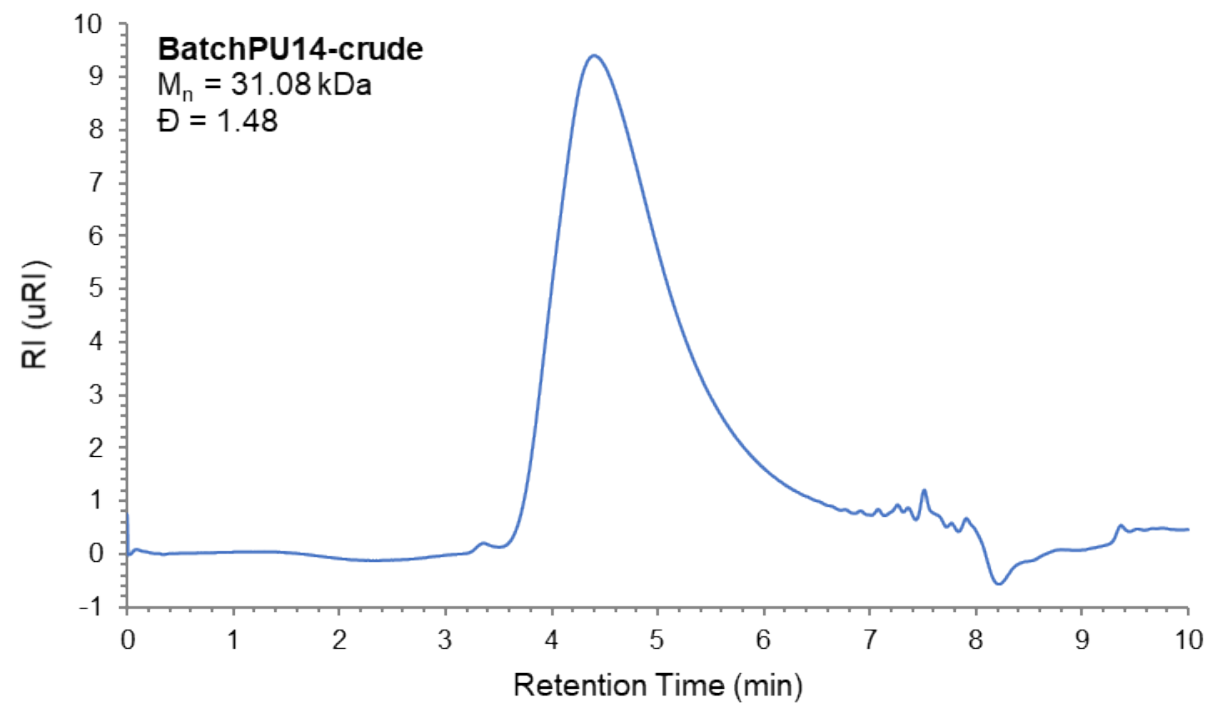

Figure S100: APC trace of BatchPU14-crude.

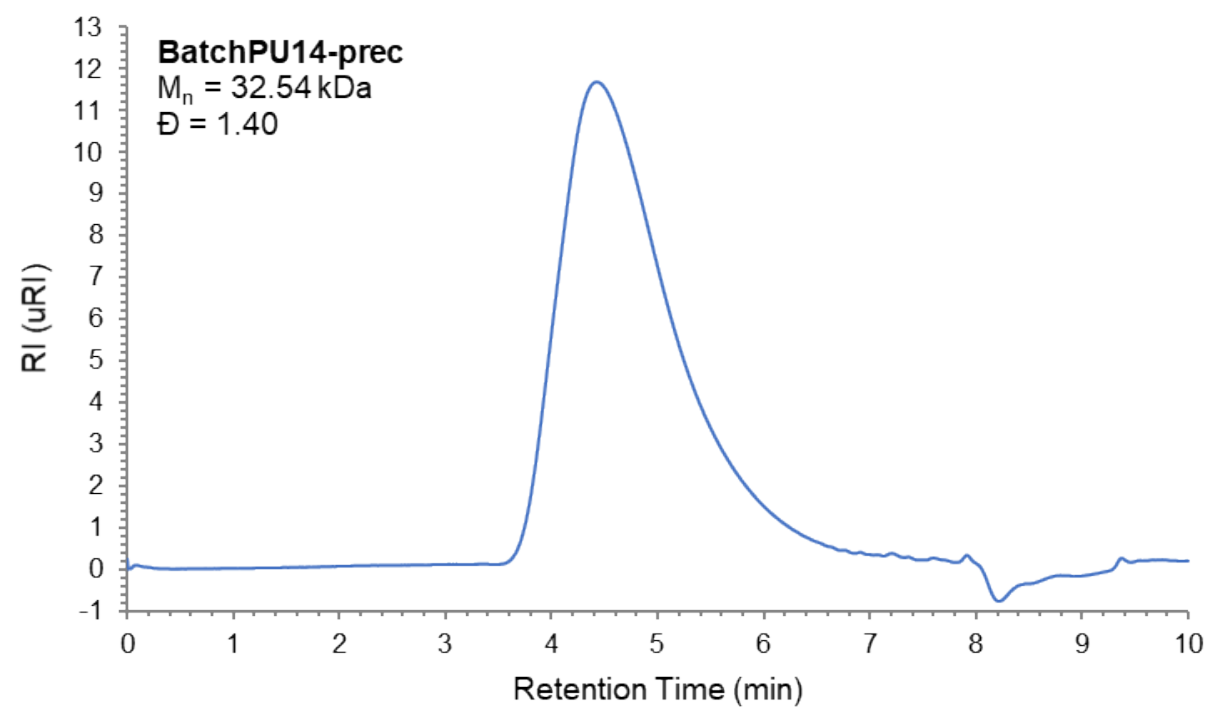

Figure S101: APC trace of BatchPU14-prec. 


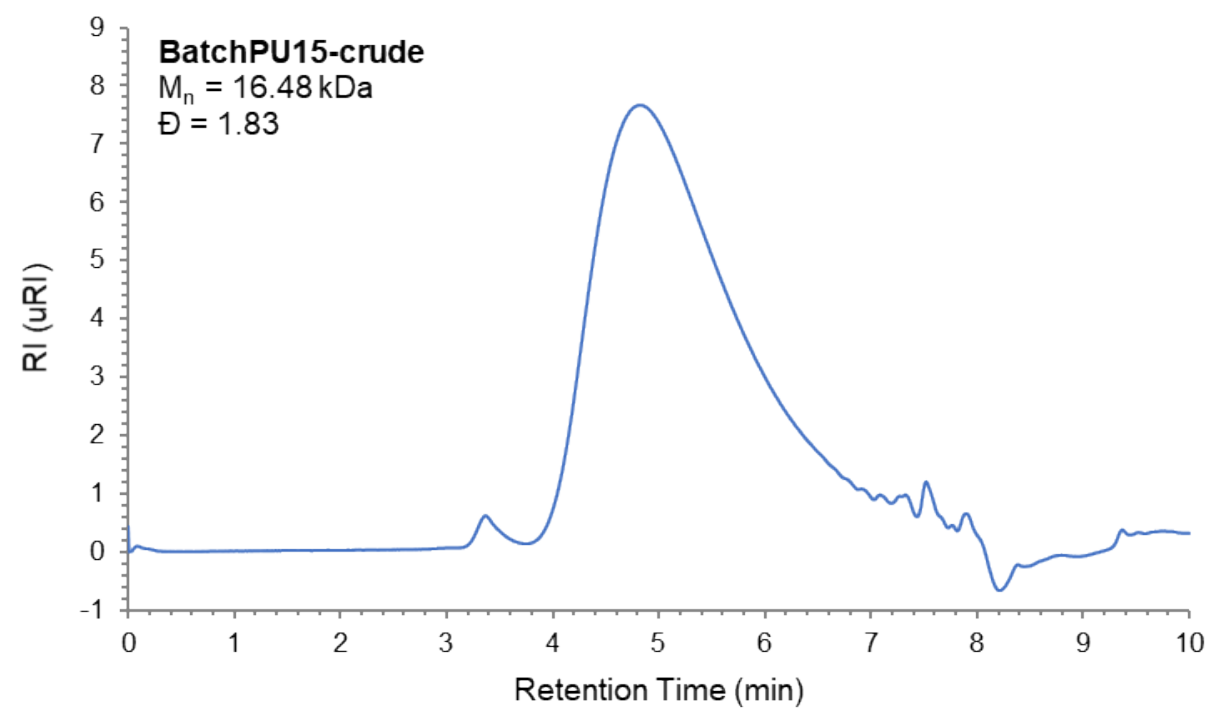

Figure S102: APC trace of BatchPU15-crude.

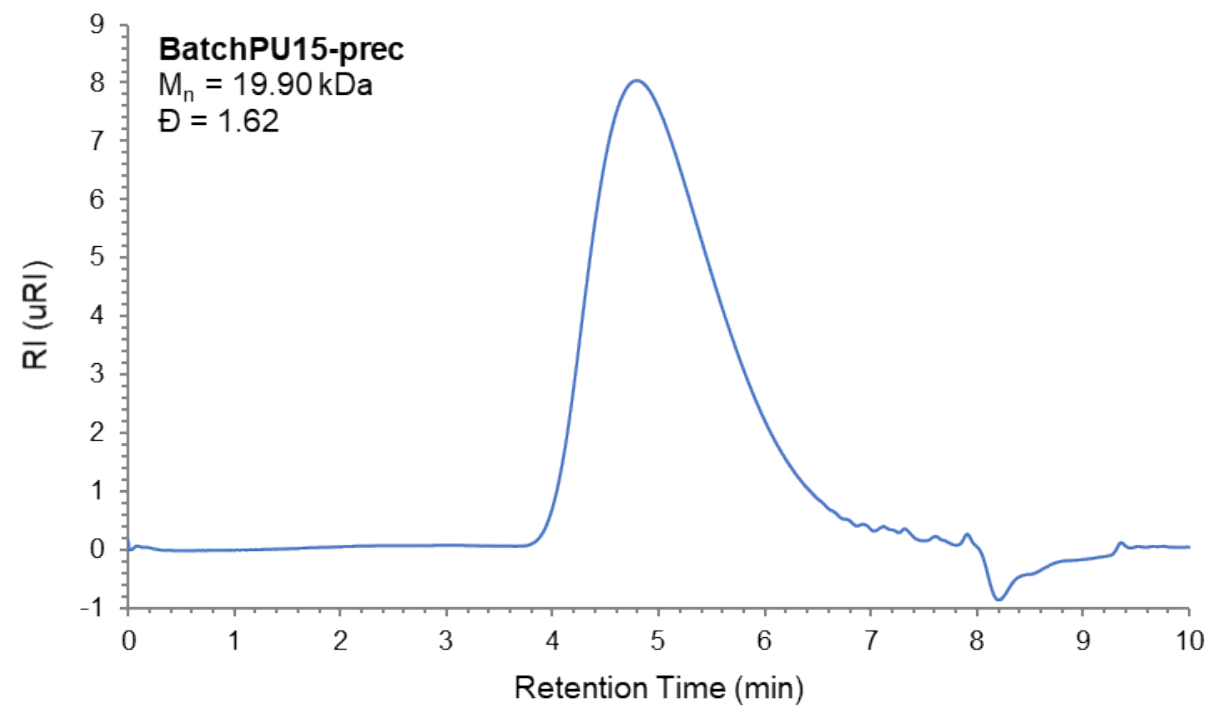

Figure S103: APC trace of BatchPU15-prec. 


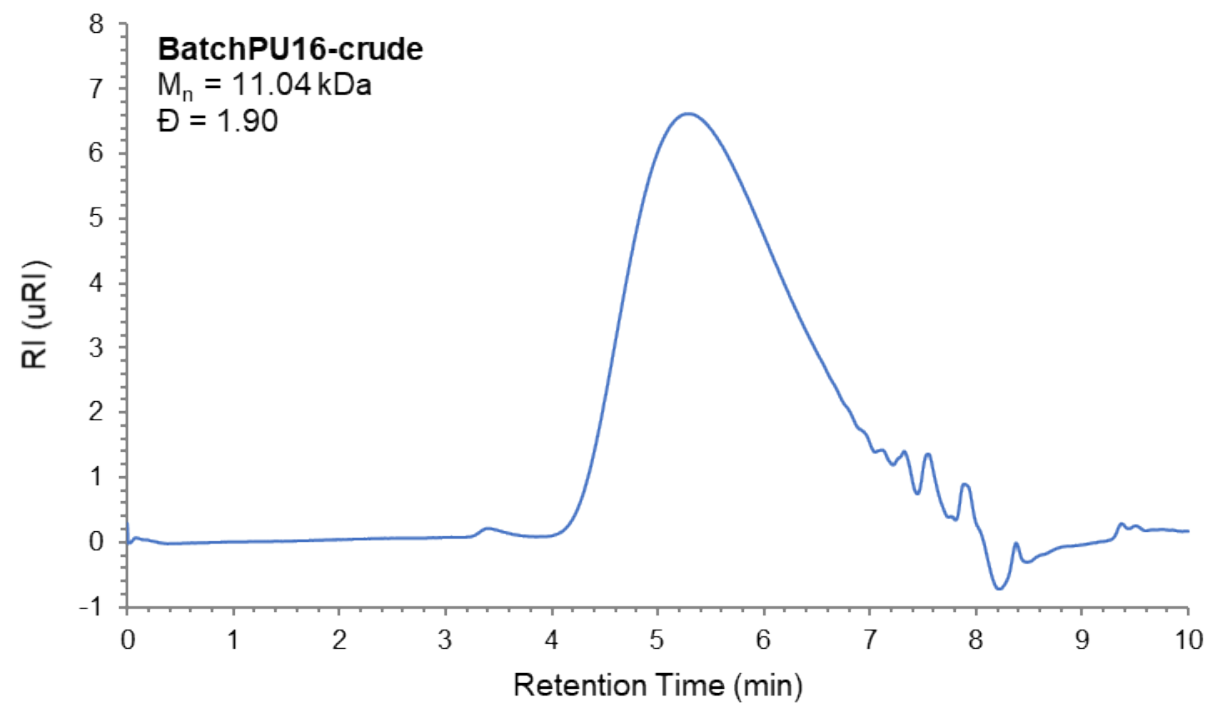

Figure S104: APC trace of BatchPU16-crude.

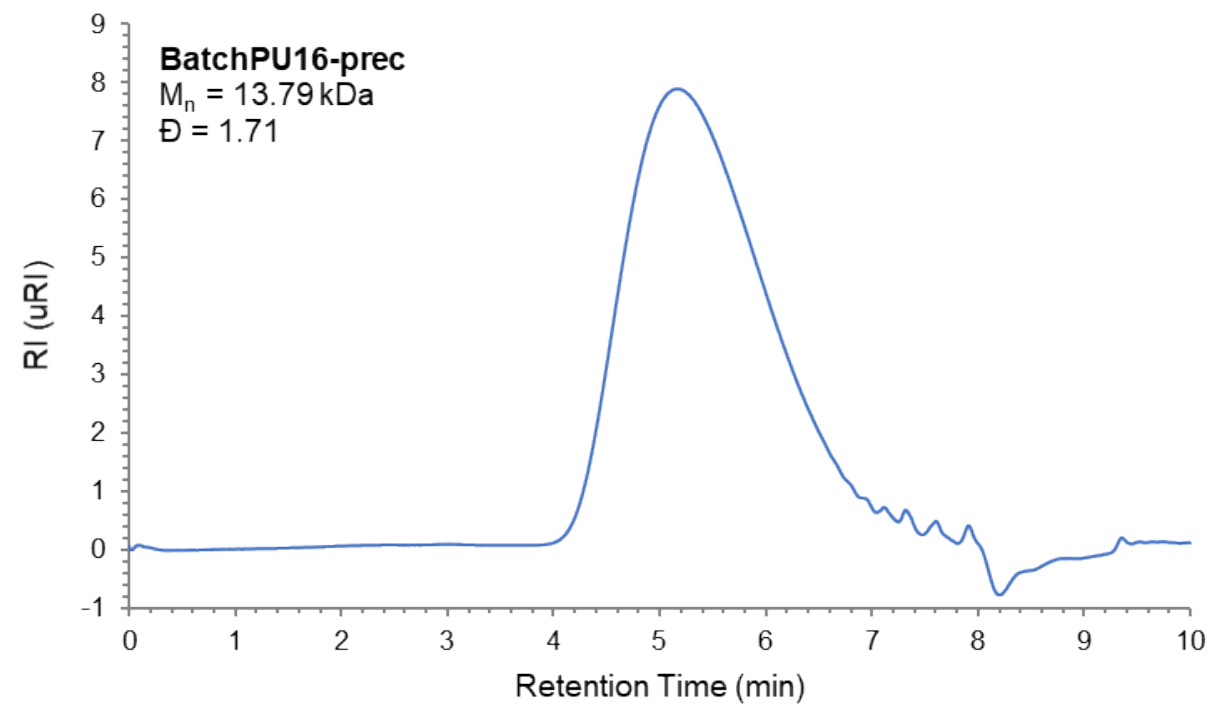

Figure S105: APC trace of BatchPU16-prec. 

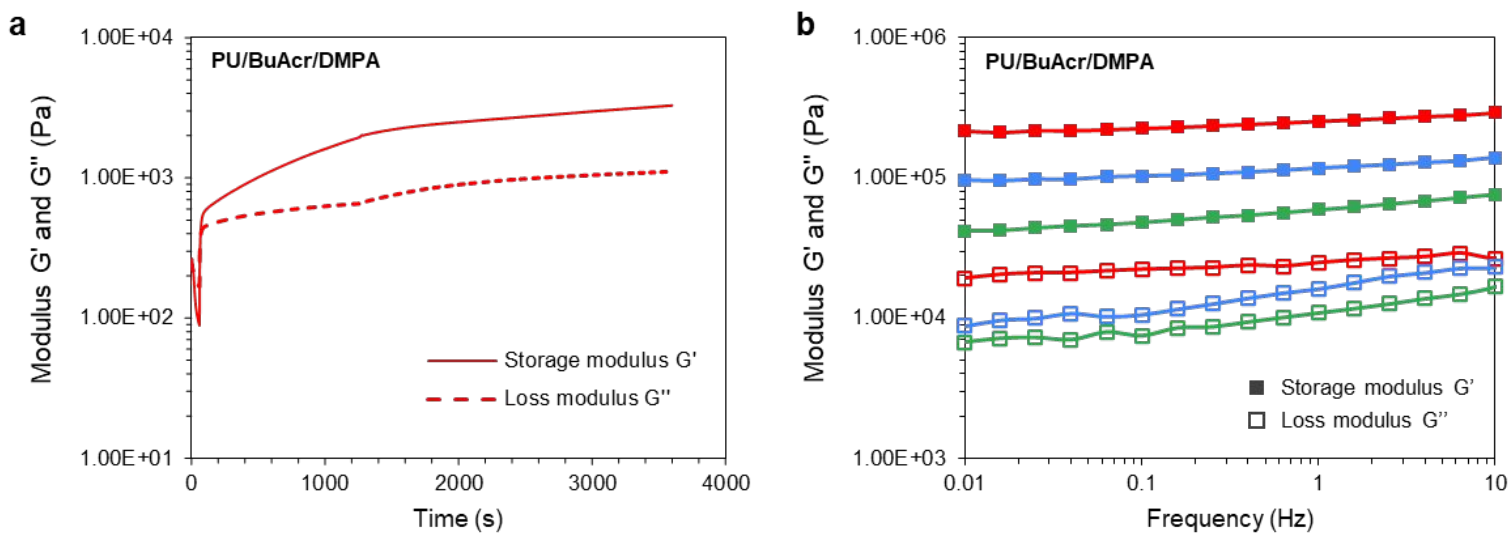

Figure S106: a) Evolution of the storage modulus G' and the loss modulus G' of the formulation containing FlowPU8, BuAcr (3:1) and DMPA as a function of irradiation time using $100 \mathrm{~mW} \cdot \mathrm{cm}^{-2}$ of irradiation intensity. b) Evolution of the storage modulus $\mathrm{G}^{\prime}$ and the loss modulus G', as a function of frequency for crosslinked samples from formulations containing FlowPU8 (red), FlowPU9 (blue), and FlowPU10 (green) and BuAcr (3:1) and DMPA.
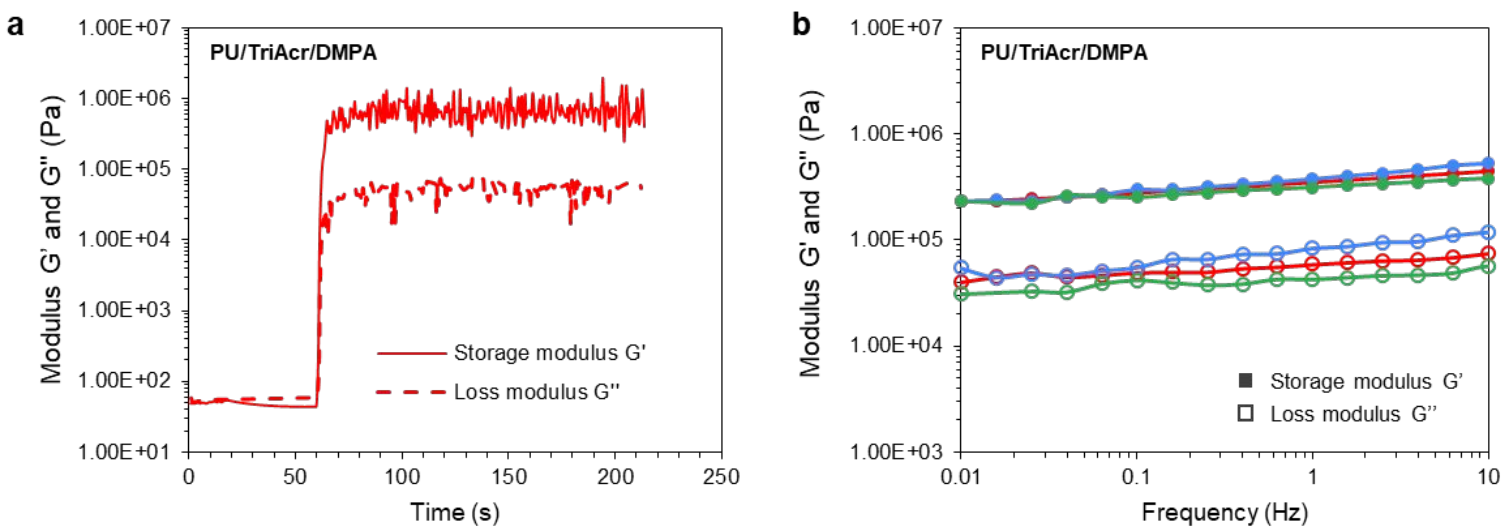

Figure S107: a) Evolution of the storage modulus $G^{\prime}$ and the loss modulus $G$ ' of the formulation containing FlowPU8, TriAcr (3:1) and DMPA as a function of irradiation time using $100 \mathrm{~mW} \cdot \mathrm{cm}^{-2}$ of irradiation intensity. b) Evolution of the storage modulus $\mathrm{G}^{\prime}$ and the loss modulus $G$ ' as a function of frequency for crosslinked samples from formulations containing FlowPU8 (red), FlowPU9 (blue), and FlowPU10 (green) and TriAcr (3:1) and DMPA. 


\section{References}

(1) Wegner, J.; Ceylan, S.; Kirschning, A. Ten Key Issues in Modern Flow Chemistry. Chem. Commun. 2011, 47 (16), 4583-4592. https://doi.org/10.1039/c0cc05060a.

(2) Lin, B.; Hedrick, J. L.; Park, N. H.; Waymouth, R. M. Programmable HighThroughput Platform for the Rapid and Scalable Synthesis of Polyester and Polycarbonate Libraries. J. Am. Chem. Soc. 2019, 141 (22), 8921-8927. https://doi.org/10.1021/jacs.9b02450.

(3) Tonhauser, C.; Natalello, A.; Löwe, H.; Frey, H. Microflow Technology in Polymer Synthesis. Macromolecules 2012, 45 (24), 9551-9570. https://doi.org/10.1021/ma301671x.

(4) Shashi Menon, E. Fluid Flow in Pipes. In Transmission Pipeline Calculations and Simulations Manual; Elsevier, 2015; pp 149-234. https://doi.org/10.1016/b978-185617-830-3.00005-5.

(5) LaNasa, P. J.; Upp, E. L. Basic Flow Measurement Laws. In Fluid Flow Measurement; Elsevier, 2014; pp 19-29. https://doi.org/10.1016/b978-0-12-409524-3.00002-2.

(6) Rehm, B.; Consultant, D.; Haghshenas, A.; Paknejad, A. S.; Schubert, J. Situational Problems in MPD. In Managed Pressure Drilling; Elsevier, 2008; pp 39-80. https://doi.org/10.1016/b978-1-933762-24-1.50008-5.

(7) Odian, G. PRINCIPLES OF POLYMERIZATION, 4th Editio.; John Wiley \& Sons, Inc.: HOBOKEN, NJ, 2004.

(8) Harrell, M. L.; Bergbreiter, D. E. Using 1 H NMR Spectra of Polymers and Polymer Products To Illustrate Concepts in Organic Chemistry. J. Chem. Educ. 2017, No. 94, 1668-1673. https://doi.org/10.1021/acs.jchemed.6b00801.

(9) Zdrahala, R. J.; Gerkin, R. M.; Hager, S. L. Polyether-Based Thermoplastic 
Polyurethanes. I. Effect of the Hard-Segment Content. 2000, 24 (1979), 2041-2050.

(10) Koltzenburg, S.; Maskos, M.; Nuyken, O. Polymer Chemistry; Springer Berlin Heidelberg: Berlin, Heidelberg, 2017. https://doi.org/10.1007/978-3-662-49279-6.

(11) Gavade, S.; Balaskar, R.; Mane, M.; Pabrekar, P. N.; Mane, D. Cu(Acac)2-Catalyzed N-Arylations of Phenylurea with Aryl Boronic Acid. Synth. Commun. 2012, 42 (11), 1704-1714. https://doi.org/10.1080/00397911.2010.543747.

(12) Malkin, A. Y.; Sherysheva, L. I.; Kulichikhin, S. G.; Zhirkov, P. V. The Flow of a Polymerizing Liquid in a Cylindrical Pipe. Polym. Eng. Sci. 1983, 23 (15), 804-809. https://doi.org/10.1002/pen.760231503.

(13) Vrentas, J. S.; Huang, W. J. Radial Transport in Tubular Polymerization Reactors. Chem. Eng. Sci. 1986, 41 (8), 2041-2051. https://doi.org/10.1016/00092509(86)87120-2.

(14) Tyreus, B. D.; Luyben, W. L. Unusual Dynamics of a Reactor/Preheater Process with Deadtime, Inverse Response and Openloop Instability. J. Process Control 1993, 3 (4), 241-251. https://doi.org/10.1016/0959-1524(93)80030-F.

(15) Waller, K. V. T.; Nygárdas, C. G. On Inverse Repsonse in Process Control. Ind. Eng. Chem. Fundam. 1975, 14 (3), 221-223. https://doi.org/10.1021/i160055a014.

(16) Inoya, K.; Altpeter, R. J. Inverse Response in Process Control. Ind. Eng. Chem. 1962, 54 (7), 39-43. https://doi.org/10.1021/ie50631a007. 